Check for updates

Cite this: Chem. Soc. Rev., 2020, 49, 1253

Received 3rd November 2019

DOI: $10.1039 / c 9 c s 00283 a$

rsc.li/chem-soc-rev

\section{The versatile biomedical applications of bismuth- based nanoparticles and composites: therapeutic, diagnostic, biosensing, and regenerative properties}

\author{
Mohammad-Ali Shahbazi, (D) *ab Leila Faghfouri, ${ }^{b}$ Mónica P. A. Ferreira, ${ }^{a}$ \\ Patrícia Figueiredo, ${ }^{a}$ Hajar Maleki, (DD ${ }^{c}$ Farshid Sefat, ${ }^{\text {de }}$ Jouni Hirvonen ${ }^{a}$ and \\ Hélder A. Santos (D) *af
}

\begin{abstract}
Studies of nanosized forms of bismuth (Bi)-containing materials have recently expanded from optical, chemical, electronic, and engineering fields towards biomedicine, as a result of their safety, cost-effective fabrication processes, large surface area, high stability, and high versatility in terms of shape, size, and porosity. $\mathrm{Bi}$, as a nontoxic and inexpensive diamagnetic heavy metal, has been used for the fabrication of various nanoparticles (NPs) with unique structural, physicochemical, and compositional features to combine various properties, such as a favourably high X-ray attenuation coefficient and near-infrared (NIR) absorbance, excellent light-to-heat conversion efficiency, and a long circulation half-life. These features have rendered bismuth-containing nanoparticles (BiNPs) with desirable performance for combined cancer therapy, photothermal and radiation therapy (RT), multimodal imaging, theranostics, drug delivery, biosensing, and tissue engineering. Bismuth oxyhalides $\left(\mathrm{BiO}_{x}\right.$, where $\mathrm{X}$ is $\mathrm{Cl}, \mathrm{Br}$ or $\left.\mathrm{I}\right)$ and bismuth chalcogenides, including bismuth oxide, bismuth sulfide, bismuth selenide, and bismuth telluride, have been heavily investigated for therapeutic purposes. The pharmacokinetics of these BiNPs can be easily improved via the facile modification of their surfaces with biocompatible polymers and proteins, resulting in enhanced colloidal stability, extended blood circulation, and reduced toxicity. Desirable antibacterial effects, bone regeneration potential, and tumor growth suppression under NIR laser radiation are the main biomedical research areas involving BiNPs that have opened up a new paradigm for their future clinical translation. This review emphasizes the synthesis and state-of-the-art progress related to the biomedical applications of BiNPs with different structures, sizes, and compositions. Furthermore, a comprehensive discussion focusing on challenges and future opportunities is presented.
\end{abstract}

\section{Introduction}

Given the importance of human life, scientists have always sought to promote the conventional treatment methodologies and diagnosis approaches of various diseases. Despite many

\footnotetext{
${ }^{a}$ Drug Research Program, Division of Pharmaceutical Chemistry and Technology, Faculty of Pharmacy, FI-O0014 University of Helsinki, Helsinki, Finland. E-mail:m.a.shahbazi@helsinki.fi

${ }^{b}$ Department of Pharmaceutical Nanotechnology, School of Pharmacy, Zanjan University of Medical Sciences, 56184-45139 Zanjan, Iran

${ }^{c}$ Institute of Inorganic Chemistry, Department of Chemistry, University of Cologne, Cologne, Germany

${ }^{d}$ Department of Biomedical and Electronics Engineering, School of Engineering, University of Bradford, Bradford, UK

${ }^{e}$ Interdisciplinary Research Centre in Polymer Science \& Technology (IRC Polymer), University of Bradford, Bradford, UK

${ }^{f}$ Helsinki Institute of Life Science (HiLIFE), University of Helsinki, FI-00014

Helsinki, Finland.E-mail: helder.santos@helsinki.fi
}

attempts, unresolved challenges have still remained for hardto-treat diseases, including cancer, resistant bacterial infections, heart diseases, as well as organ dysfunction disorders, resulting in a high annual mortality rate. ${ }^{1-6}$ As a solution, nanoscience researchers are always looking for efficient particles that can lead to the development of new medicines to assist in early diagnosis and the therapy of life-threatening diseases. ${ }^{7}$ There are plenty of organic and inorganic nanocarriers under investigation for such purposes. ${ }^{8-17}$ Among them, bismuthcontaining nanoparticles (BiNPs), although in their infancy, have recently garnered much attention as a research breakthrough for biomedical applications, owing to their excellent properties, which include high stability, high surface area, strong diamagnetism, high electrical and magnetoresistance when placed in a magnetic field, desirable catalytic activity, ease of functionalization, cost-effectiveness, chemical inertness, low toxicity, high X-ray attenuation coefficient, strong near-infrared (NIR) 
absorbance, high photothermal-conversion efficiency, and favorable antibacterial activity. ${ }^{18-20}$ Moreover, $\mathrm{Bi}$ is considered as one of the least toxic and biologically non-reactive heavy metals, which is more suitable for in vivo applications compared to other metals such as silver. ${ }^{12}$ The ease in controlling their particle size and shape during synthesis is another advantage of BiNPs, which can open new opportunities for its future clinical applications. ${ }^{21}$ All of the abovementioned benefits contribute to the increasing interest in the biomedical applications of BiNPs over other metal-based NPs.

While Bi has been conventionally used in the production of pharmaceutical products for the treatment of gastrointestinal disorders, hypertension, and syphilis, its usage in nanostructural forms has been widely expanded in recent years in various domains, such as X-ray radiotherapy (RT), biosensors, heavymetal ion detectors, antimicrobial formulations, combined cancer therapy, bioimaging, and tissue engineering (Fig. 1). ${ }^{22-25}$ For these applications, single-component NPs of Bi can be used as intermediates to fabricate other types of BiNPs, including $\mathrm{Bi}$ oxyhalides (BiOX, where $\mathrm{X}$ is $\mathrm{Cl}, \mathrm{Br}$ or $\mathrm{I}$ ) and $\mathrm{Bi}$ chalcogenides (e.g., Bi oxide $\left(\mathrm{Bi}_{2} \mathrm{O}_{3}\right)$, Bi sulfide $\left(\mathrm{Bi}_{2} \mathrm{~S}_{3}\right)$, Bi selenide $\left(\mathrm{Bi}_{2} \mathrm{Se}_{3}\right)$, and $\mathrm{Bi}$ telluride $\left.\left(\mathrm{Bi}_{2} \mathrm{Te}_{3}\right)\right)$. The nanostructures of such Bi-based compounds may adopt different morphologies, such as nanotubes, nanowires, nanorods, nanoflowers, nanoneedles, nanoflakes, nanoplates, nanosheets, and nanooctahedra. Other types of multi-component Bi nanostructures have also been fabricated and used for biomedical applications, including $\mathrm{Bi}$ ferrite $\left(\mathrm{BiFeO}_{3}\right)$, $\mathrm{Bi}$ tungstate $\left(\mathrm{Bi}_{2} \mathrm{WO}_{6}\right), \mathrm{Bi}$ molybdate $\left(\mathrm{Bi}_{2} \mathrm{MoO}_{6}\right), \mathrm{Bi}$ vanadate $\left(\mathrm{BiVO}_{4}\right), \mathrm{Bi}$ phosphate $\left(\mathrm{BiPO}_{4}\right)$, Bi oxide carbonate $\left(\left(\mathrm{Bi}_{2} \mathrm{O}\right)_{2} \mathrm{CO}_{3}\right)$, and $\mathrm{Bi}$ dimercaptopropanol (BisBAL). The methods developed for the synthesis of these NPs include hydrothermal or solvothermal syntheses, evaporation methods, sol-gel approaches, microemulsion techniques, chemical synthesis, microwave irradiation, sonochemical synthesis, and laser-mediated approaches. ${ }^{26-32}$

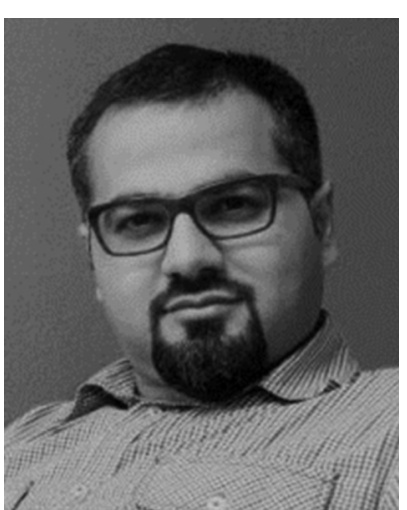

Mohammad-Ali Shahbazi
Mohammad-Ali Shahbazi received his PhD in 2015 from University of Helsinki, Finland, where he worked on porous materials for drug delivery to cancer tissues. $\mathrm{He}$ is currently a postdoc scientist at Faculty of Pharmacy, University of Helsinki, working on therapeutic microdevices for autoimmune diseases. Dr Shahbazi is also an expert in oral peptide delivery and fabrication of cellmimicking carriers. He has authored more than 70 scientific articles with the aim of shaping the future of multifunctional medicines through a combination of materials science and nanotechnology. His current research interest lies in nano-based regenerative hydrogels for wound healing, bone repair and long-term drug delivery.
In biomedicine, one of the main research areas of BiNPs is cancer therapy. While common therapies employed for cancer, including surgery, chemotherapy and RT, have constantly advanced over the decades, this disease is still one of the leading causes of human death and intensive side effects of the conventional monotherapies have remained unresolved. ${ }^{33-35}$ BiNPs have stimulated an upsurge in interest within the area of synergistic cancer therapy due to their remarkable absorbance of NIR light, high light-to-heat conversion efficiency, and excellent photothermal stability, meaning that they can be used for photothermal therapy (PTT) in combination with chemotherapy, radiotherapy, and immunotherapy. ${ }^{36-38}$ RT of cancer is also possible using BiNPs as a potential radiosensitizer, with better performance compared to gold (Au) and germanium (Ge) NPs. High-intensity focused ultrasound (HIFU) therapy of cancer using BiNPs has also been reported. ${ }^{39}$ Nevertheless, the lack of early diagnosis might hinder the efficient killing of cancer cells by such NPs. Therefore, there has been exponential growth in the use of BiNPs as excellent contrast agents for the early diagnosis of cancer through different imaging modalities, such as computerized tomography (CT)-scan examinations and photoacoustic (PA) imaging at a very low dose, allowing more flexibility in a clinical setting. ${ }^{40-43}$ In addition, BiNP-mediated infrared thermography (IRT) can also be employed as an imaging method to monitor tumor temperature variations during PTT. The suitability of Bi for CT-imaging is due to its high K-edge value (K-edge value $=90.5 \mathrm{keV}$ ) and large $\mathrm{X}$-ray attenuation coefficient $\left(5.74 \mathrm{~cm}^{2} \mathrm{~g}^{-1}\right.$ at $\left.100 \mathrm{keV}\right)$, which is greater than those of other high- $Z$ materials. ${ }^{44}$ Its appropriate PA and IRT imaging resolutions are also due to its desirable photothermal conversion efficiency in the NIR window. ${ }^{43}$ Moreover, some researchers have greatly broadened the application of BiNPs by integrating new functional components into their structure, paving the way for new applications of BiNPs. For example, the integration of

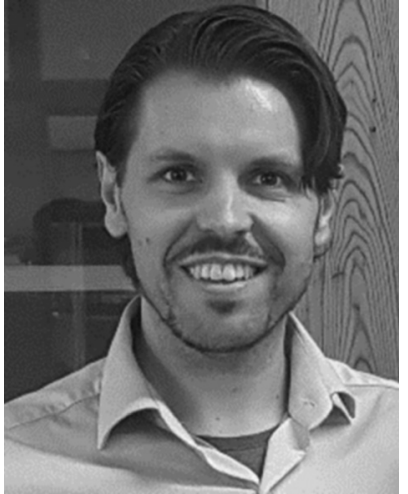

Hélder A. Santos
Hélder A. Santos obtained his Doctor of Science in Technology (Chemical Engineering) in 2007 from the Helsinki University of Technology. Currently, he is an Associated Professor at the Faculty of Pharmacy, University of Helsinki, Head of the Division of Pharmaceutical Chemistry and Technology, Head of the Preclinical Drug Formulation and Analysis Group, and Head of the Nanomedicines and Biomedical Engineering research group. His scientific expertise lies in the development of nanoparticles/ nanomedicines for biomedical applications, particularly porous silicon and polymeric-based nanomaterials for simultaneous controlled drug delivery, diagnostics, and therapy for cancer, diabetes, and cardiovascular diseases. 


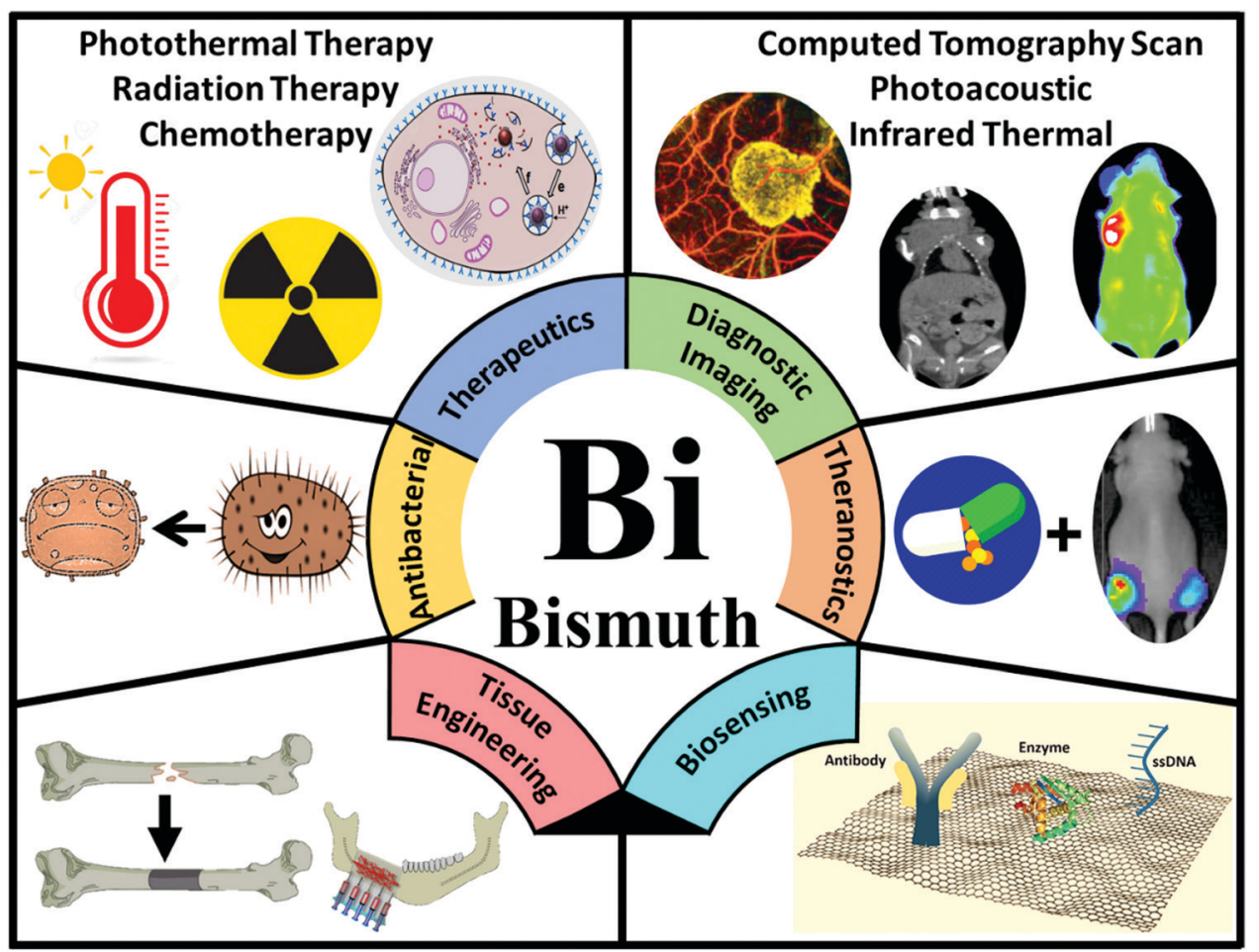

Fig. 1 A schematic overview of the emerging biomedical applications of BiNPs. The main biomedical applications include drug delivery and cancer therapy, bioimaging, the development of theranostic NPs, antibacterial uses, regenerative medicine (particularly bone engineering), and the fabrication of biosensors.

manganese (Mn), iron (Fe), and gadolinium (Gd) with BiNPs has resulted in the NPs becoming a contrast agent for MRI. ${ }^{45-48}$ The imaging potential of BiNPs can be combined with its PTT effect and other therapeutic approaches, such as immunotherapy and chemotherapy, to develop theranostic Bi-based medicines that satisfy strict demands for multimodal imagingguided synergistic therapies and overcome the deficiencies of single imaging methods and monotherapy of cancer. ${ }^{49}$ Promising outcomes using theranostic BiNPs have been obtained for thermo-chemotherapy ${ }^{50,51}$ chemo-radiotherapy, ${ }^{52}$ thermo-radiotherapy, ${ }^{53,54}$ and radio-immunotherapy ${ }^{55}$ with significantly higher inhibition of cancer cells, compared to available monotherapies. In biosensing applications, BiNPs have also opened up a new gateway as promising materials to fabricate biologically compatible devices for the rapid and easy detection of biological events. For example, $\mathrm{Bi}_{2} \mathrm{WO}_{6} \mathrm{NPs}$ with multilayer reduced graphene nanosheets as a modified electrode have been reported for the rapid electrochemical sensing of oxidative stress in biological samples. ${ }^{56}$

In this review, we will provide a comprehensive overview of the recently generated information on the synthesis, biodistribution, safety, as well as modifications conducted on various types of BiNPs to produce multifunctional materials for different biomedical applications that have caught the interest of the scientific community. The available literature suggests that biomedical research on BiNPs has been extensively growing and various nanoforms of $\mathrm{Bi}$ with different compositions have been exploited for biological applications, including drug delivery, antimicrobial activity, bioimaging, cancer therapy, biosensing, and tissue engineering. Nevertheless, to the best of our knowledge, there has been no comprehensive review on such features of BiNPs. Therefore, we will discuss all representative studies exploiting BiNPs for the abovementioned biomedical applications. Future perspectives in the development of these NPs for biomedical applications are also proposed.

\section{Principles and innovations in the synthesis of BiNPs}

BiNPs have attracted increased attention for biomedical applications due to their low toxicity and environmentally friendly properties. ${ }^{57}$ Moreover, the relatively low price and abundancy of $\mathrm{Bi}$ is attractive for its large-scale applications. ${ }^{58}$ Bulk Bi is a semimetal with large Fermi wavelengths, high magnetoresistance, and strong diamagnetism, ${ }^{59}$ which can be used to fabricate BiNPs with different shapes and compositions. The most common ones are $\mathrm{Bi}_{2} \mathrm{O}_{3}, \mathrm{Bi}_{2} \mathrm{~S}_{3}, \mathrm{Bi}_{2} \mathrm{Se}_{3}$, and $\mathrm{Bi}_{2} \mathrm{Te}_{3}$ that belong to the group VI of $\mathrm{Bi}$ compounds, so-called $\mathrm{Bi}$ chalcogenides. The nanostructures of $\mathrm{Bi}$ chalcogenides exhibit intrinsic electronic and optical properties, which make them suitable for a wide range of biomedical applications; ${ }^{60}$ however, these properties are influenced by their morphology and crystal structure. ${ }^{61} \mathrm{Bi}$ oxyhalides (BiOX, where $\mathrm{X}$ is $\mathrm{Cl}$, $\mathrm{Br}$, or I) are another class of Bi compounds that belong to the V-VI-VII ternary oxide semiconductor materials. Due to their 
layered structure and high chemical stability, along with their optical, electrical, and mechanical properties, these types of materials have mainly attracted increased attention for photocatalytic activities under visible light irradiation, as well as electronics, and energy storage. ${ }^{58,62-64}$ Other types of Bi nanostructures have also been fabricated, including $\mathrm{BiFeO}_{3}, \mathrm{Bi}_{2} \mathrm{WO}_{6}$, $\mathrm{Bi}_{2} \mathrm{MoO}_{6}, \mathrm{BiVO}_{4}, \mathrm{BiPO}_{4},\left(\mathrm{Bi}_{2} \mathrm{O}\right)_{2} \mathrm{CO}_{3}$, and BisBAL. ${ }^{24,65-70}$ The main characteristics, properties, and applications of the most common types of BiNPs are summarized in Table 1.

Through the adjustment of various parameters during the fabrication process, Bi nanostructures with different morphologies can be prepared, including: (i) 0D nanostructures, such as nanospheres, and nanocubes; (ii) 1D nanostructures, including nanowires, nanorods, and nanotubes; and (iii) 2D nanostructures, such as nanoplates, nanosheets, and thin films. ${ }^{111}$ Increased efforts have been made to fabricate the above morphologies of BiNPs using different methodologies, such as hydrothermal or solvothermal synthesis, evaporation methods, sol-gel approaches, microemulsion techniques, chemical synthesis, microwave irradiation, sonochemical synthesis, and lasermediated approaches. ${ }^{65,112-119}$ In this section, the most common approaches introduced for the fabrication of BiNPs will be discussed, and the principals of each technique will be detailed, along with the influence of the different synthesis parameters on the morphology of the Bi nanostructures, and consequently, on their properties. The different fabrication methods of the main BiNPs, the treatment conditions applied to prepare these structures, and their size, shape, properties and possible applications are summarized in Table 2.

\subsection{Hydrothermal/solvothermal/ionothermal synthesis of BiNPs}

Hydrothermal synthesis of NPs has been employed for the fabrication of different inorganic materials, such as BiNPs. Generally, hydrothermal synthesis involves crystal growth in aqueous solutions under high temperatures (not more than $300{ }^{\circ} \mathrm{C}$ ) and high pressure, in which the substances are not soluble at normal temperatures and pressures $\left(100{ }^{\circ} \mathrm{C},<1 \mathrm{~atm}\right) .{ }^{164}$ The reaction is usually conducted in a metallic sealed reactor, a so-called autoclave, made of Teflon and coated with platinum, gold, or silver to protect the reactor from highly corrosive solvents. ${ }^{165}$ The hydrothermal process presents energy saving and cost-effective benefits in fabricating BiNPs, with controllable particle size, morphology and degree of crystallinity by changing the concentration of the Bi source. ${ }^{125}$ Furthermore, the processing temperature and reaction time can be lowered using a microwave-assisted hydrothermal approach, allowing uniform nucleation of the powders in suspension. ${ }^{166}$ Additionally, by controlling the temperature, reaction time, and the $\mathrm{pH}$ value of the solution, the morphologies of the BiNPs can be tuned. For example, Deng et al. ${ }^{141}$ showed that applying a lower temperature and shorter reaction time favored the formation of nanotubes of Bi-oxyhalide NPs, while higher temperatures and longer reaction times led to the formation of more stable $\mathrm{Bi}$ oxyhalide NPs with nanobelt or nanoflake-like shapes. Additionally, higher temperatures for the hydrothermal reaction usually favor the preparation of uniform Bi structures, which can be ascribed to the increased crystal growth rate at a higher temperature. ${ }^{158}$ In another study, Cui et al. ${ }^{150}$ demonstrated that the size, shape and photocatalytic activity of $\mathrm{Bi}_{2} \mathrm{WO}_{6}$ NPs are highly dependent on the $\mathrm{pH}$ value ( $\mathrm{NaOH}$ content) of the initial synthesis solution (Fig. 2). At pH 1-4, the particles exhibited flower-like hierarchical microspheres in which the size constantly decreased when the $\mathrm{pH}$ value increased (the particle size was $7 \mu \mathrm{m}$ at $\mathrm{pH} 1$ and $1.5 \mu \mathrm{m}$ at $\mathrm{pH}$ 4). When the $\mathrm{pH}$ values were increased further from 5 to 9 , the $\mathrm{Bi}_{2} \mathrm{WO}_{6}$ particles showed irregular flake-like structures; and at $\mathrm{pH} 10-11$, the prepared $\mathrm{Bi}_{2} \mathrm{WO}_{6}$ NPs presented a uniform sphere-like morphology with an average size of $85 \mathrm{~nm}$. The flower-like microspheres prepared at pH 3 presented the highest photocatalytic activity, with 99\% degradation after $2 \mathrm{~h}$ of irradiation.

During hydrothermal synthesis, the size of the prepared particles depends on the competition between crystal nucleation and crystal growth, i.e., the crystal size is small when the rate of crystal nucleation is higher than that of the crystal growth. ${ }^{167}$ In order to control the growth speed of the prepared crystals, Wang et al. ${ }^{145}$ prepared $\mathrm{BiFeO}_{3}$ via a polymer-assisted hydrothermal process, in which poly(vinyl alcohol) (PVA) was added to prevent the crystals from growing to the micrometer size. The polymer might attach to the surface of the $\mathrm{BiFeO}_{3}$ nuclei, lowering the surface energy and the growth speed of the $\mathrm{BiFeO}_{3}$ nuclei, and therefore, restraining the size of the resulting particles to the nanometer range $(10 \mathrm{~nm})$. The addition of different surfactants can also affect the shape of the prepared nanostructures. For example, Dharmaiah et al. ${ }^{137}$ fabricated three different $\mathrm{Bi}_{2} \mathrm{Te}_{3}$ nanostructures, using ethylene glycol (EG), poly(vinyl pyrrolidone) (PVP), and ethylenediaminetetraacetic acid (EDTA) as surfactants, in order to obtain nanospheres $(25 \mathrm{~nm})$, nanoplates $(200 \mathrm{~nm})$ and nanoflakes $(300 \mathrm{~nm})$, respectively. These results suggested that surfactants can act as morphology-directing agents, regulating the crystal growth to form nanostructures with several morphologies, and subsequently affecting the physicochemical properties of the particles.

Solvothermal synthesis uses similar conditions to the hydrothermal synthesis process, but organic solvents, including toluene, decalin and, octadecene, are used instead of aqueous solutions. Similar to the hydrothermal process, the size and shape of the nanocrystals can be tuned by controlling the temperature of the reaction, and the concentration of the Bi precursors. ${ }^{168}$ Ionothermal synthesis implicates the use of ionic liquids as alternative solvent media to fabricate BiNPs. ${ }^{168}$ The main advantage of ionic liquids compared to traditional organic solvents is the low interfacial tension that leads to a high nucleation rate, and therefore, the production of very small particles. ${ }^{169}$ As demonstrated by Wang et al., the size and aspect ratio of the $\mathrm{Bi}_{2} \mathrm{~S}_{3}$ nanostructures (nanoflowers to nanorods) can be tailored by controlling the reaction time of the thermal treatment of bismuth di-n-octyl-dithiophosphate $\left(\mathrm{Bi}\left[\mathrm{S}_{2} \mathrm{P}\left(\mathrm{OC}_{8} \mathrm{H}_{17}\right)_{2}\right]_{3}\right)$ in $\mathrm{C}_{16} \mathrm{MIMBF}_{4}$ as an ionic liquid solvent, at $165{ }^{\circ} \mathrm{C}$ (Fig. 3). ${ }^{134}$ Furthermore, by changing the reaction temperature, Ma et al. fabricated $\mathrm{BiOCl}$ nanostructures with different morphologies after mixing bismuth nitrate pentahydrate 


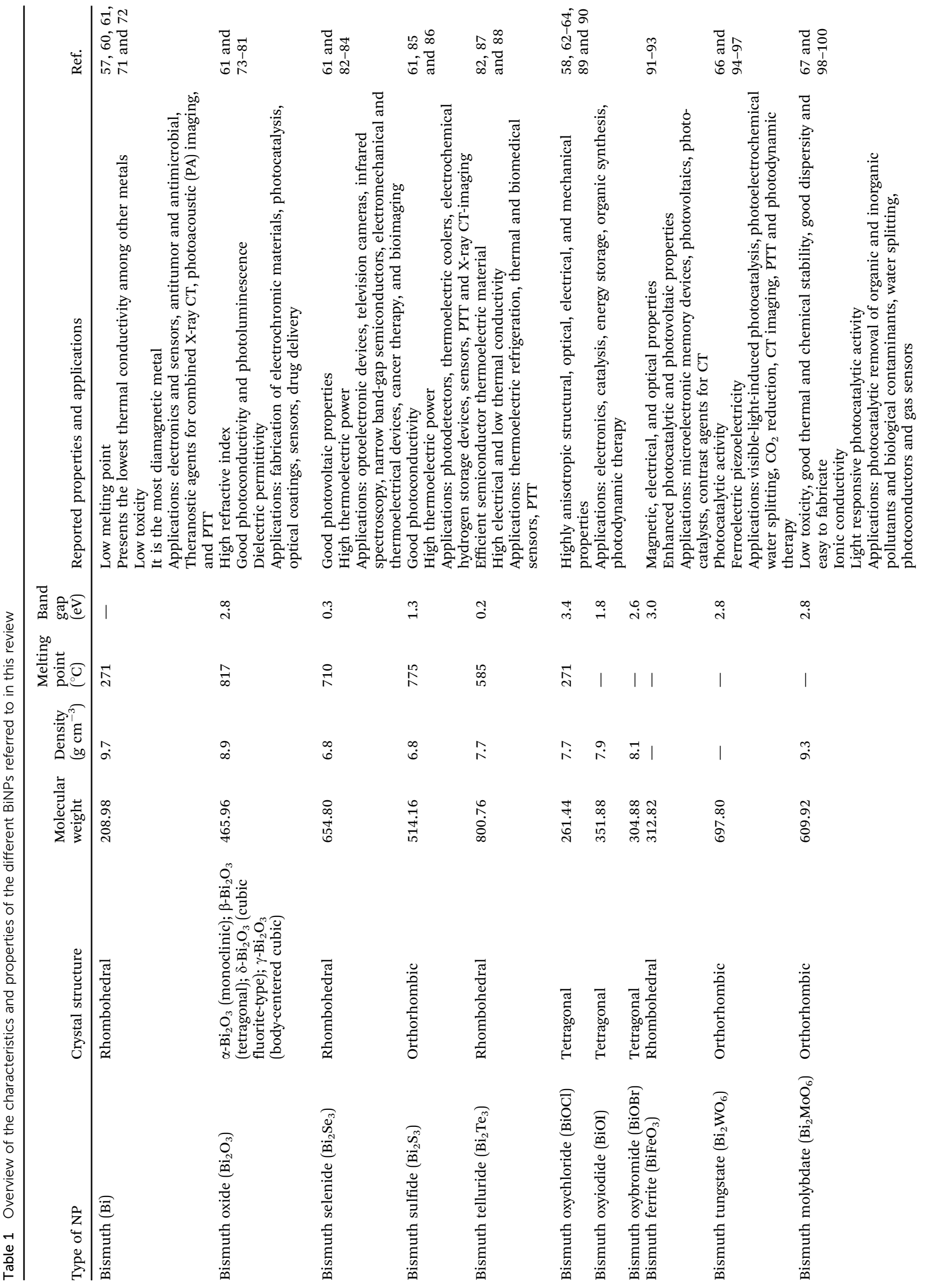


$\left[\mathrm{Bi}\left(\mathrm{NO}_{3}\right)_{3} \cdot 5 \mathrm{H}_{2} \mathrm{O}\right]$ and $\left[\mathrm{C}_{16} \mathrm{Mim}\right] \mathrm{Cl}$ as an ionic solvent for $12 \mathrm{~h}^{142}$ When the temperature was maintained at $200{ }^{\circ} \mathrm{C}$, curved nanoplates with an average thickness of around $14 \mathrm{~nm}$ where obtained, which were thinner than the nanoplates produced at $180{ }^{\circ} \mathrm{C}$. Furthermore, nanoplate arrays were obtained when a lower reaction temperature $\left(120{ }^{\circ} \mathrm{C}\right)$ was used, where the thickness of the nanoplates was $c a .32 \mathrm{~nm}$.

\subsection{Sol-gel synthesis approaches in fabricating bismuth nanostructures}

The sol-gel method is one of the most common chemical synthesis methods used to fabricate sub-micron metal oxide particles with narrow particle size distributions. ${ }^{65,168}$ This method offers good homogeneity and morphological control, lower processing temperature compared to other methods, high purity, and easy preparation of thin films and coatings. ${ }^{168}$ Using different approaches for the treatment of the sol-gel precursor, such as spin-coating, template deposition, electrospinning or heating, it is possible to obtain NPs with different morphologies, including thin films, nanotubes or nanowires, nanofibers, and spherical NPs, respectively (Fig. 4). ${ }^{65,116,170,171}$ Generally, all these approaches consist of two phases: (i) the solution phase, made up of a colloidal suspension of solid particles, and (ii) the gelation phase, where an interconnected network of solid-phase particles is formed. ${ }^{172}$ The fabrication process of the Bi nanostructures via the sol-gel method is initiated using a chemical solution that acts as the precursor, made up of a metal source, solvent and chelating agent. The process can be manipulated by changing different parameters, including the initial precursors, nature of the solvent, gelation time and conditions (e.g., temperature and $\mathrm{pH}$ ), types of additives (e.g., catalysts, surfactants and morphology directing agents), and degree of solvation. ${ }^{65,172,173}$

$\mathrm{BiFeO}_{3}$ nanostructures are commonly fabricated using the sol-gel method, where the initial precursor solution includes metal nitrates, such as $\mathrm{Bi}\left(\mathrm{NO}_{3}\right)_{3} \cdot 5 \mathrm{H}_{2} \mathrm{O}$ and $\mathrm{Fe}\left(\mathrm{NO}_{3}\right)_{3} \cdot 9 \mathrm{H}_{2} \mathrm{O}$, and an organic solvent (e.g., 2-methoxyethanol (2-MOE) or EG) that replace the water-based solvents, since metal nitrates present good solubility in these solvents. ${ }^{65,174}$ Gao et al. ${ }^{174}$ used $\mathrm{Bi}\left(\mathrm{NO}_{3}\right)_{3}$. $5 \mathrm{H}_{2} \mathrm{O}$ and $\mathrm{Fe}\left(\mathrm{NO}_{3}\right)_{3} \cdot 9 \mathrm{H}_{2} \mathrm{O}$ as starting materials and EG as a solvent, without the presence of other surfactants, in order to evaluate the effect of the calcination temperature and concentration of the precursor solution on the particle size of $\mathrm{BiFeO}_{3}$ prepared via a simple sol-gel method. Precursor solutions at concentrations of $0.3,0.4$, and $0.5 \mathrm{~mol} \mathrm{~L}^{-1}$ were stirred during $1.5 \mathrm{~h}$ at $80{ }^{\circ} \mathrm{C}$ to form gels, which were then kept at $120{ }^{\circ} \mathrm{C}$ for 4 days to obtain xerogels. Afterwards, the xerogel powders were pre-treated at $300{ }^{\circ} \mathrm{C}$ for $4 \mathrm{~h}$, before calcination treatment at 500 , 550 and $600{ }^{\circ} \mathrm{C}$ for $2 \mathrm{~h}$. The size of the prepared particles ranged between 50 and $250 \mathrm{~nm}$, which increased with the concentration of the precursor solution, due to their aggregation into bigger particles at a higher concentration of the precursor solution. An increase in the annealing temperature also translated into an increase in the particle size. Additionally, they found that a higher visible-light response, due to a smaller band gap, seemed to be the main factor for superior photocatalytic efficiency when the $\mathrm{BiFeO}_{3}$ particles were similar in size. 


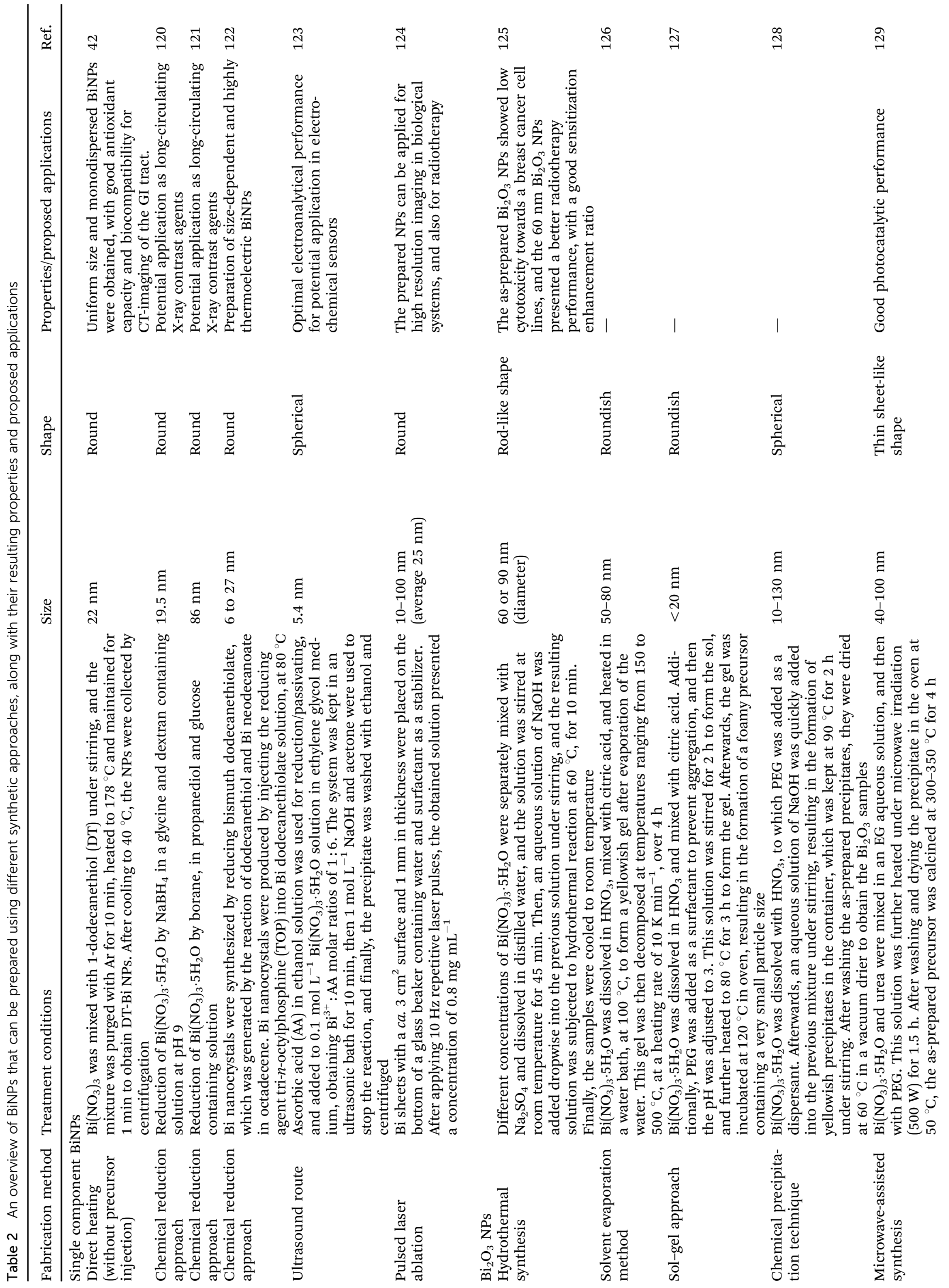




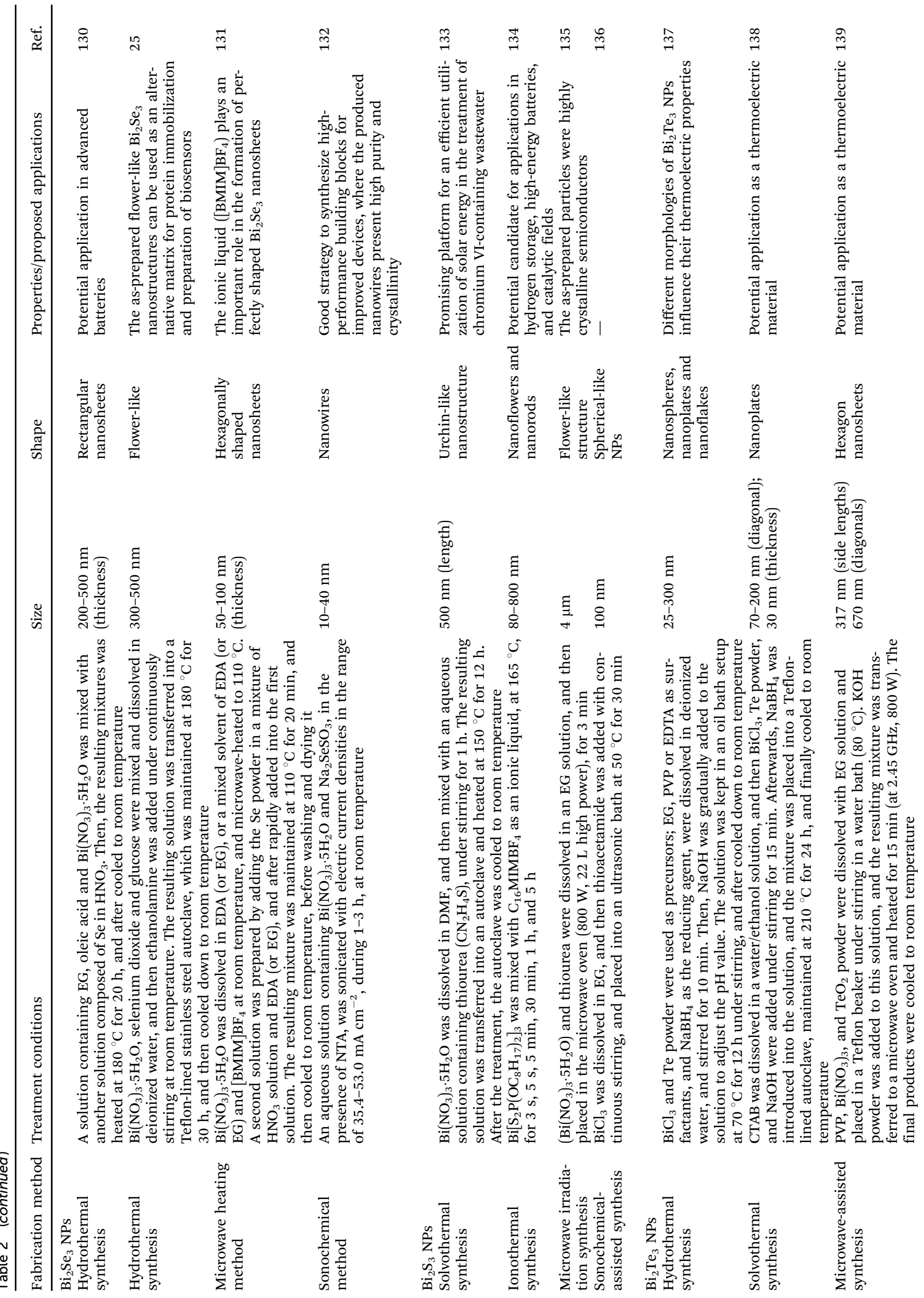




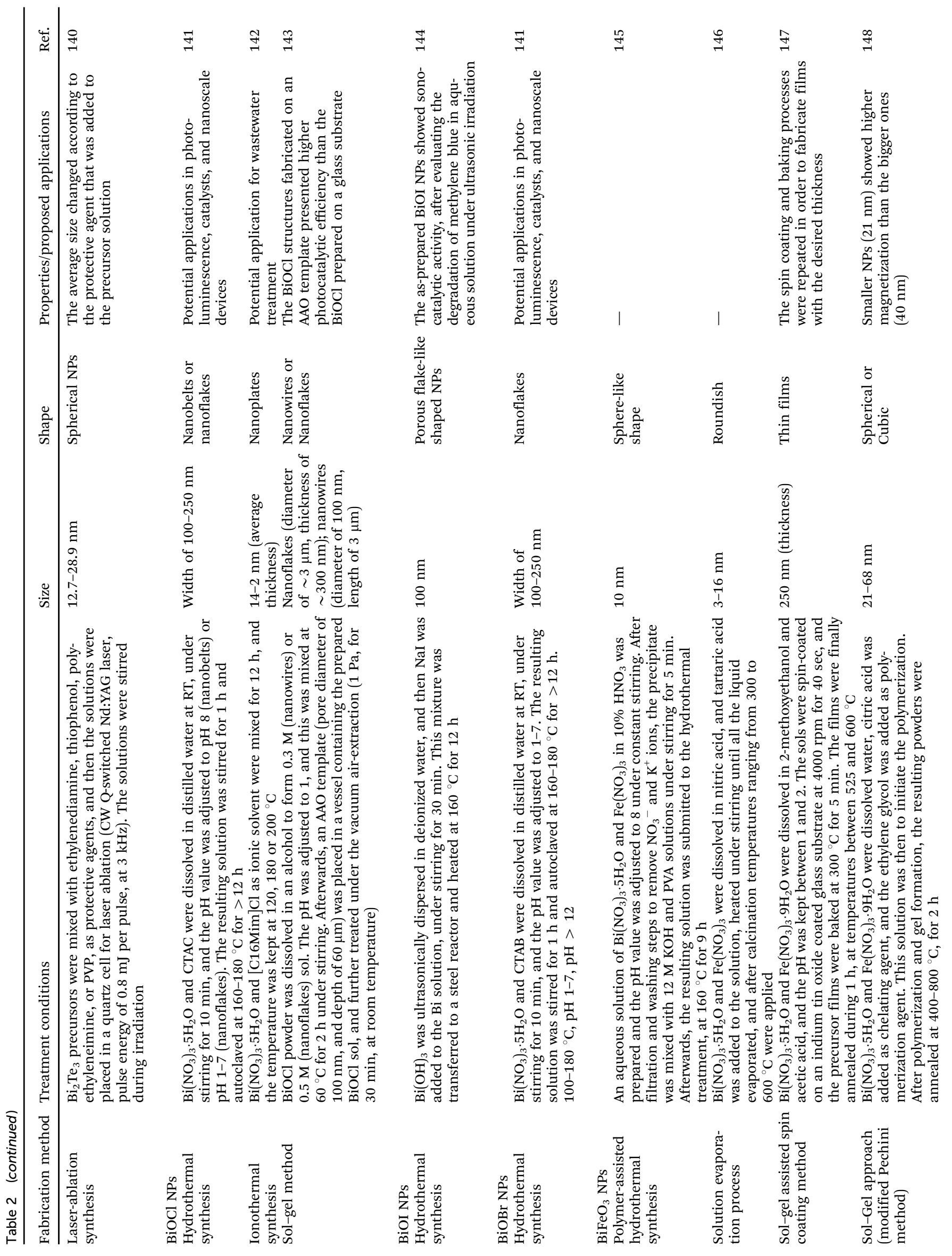




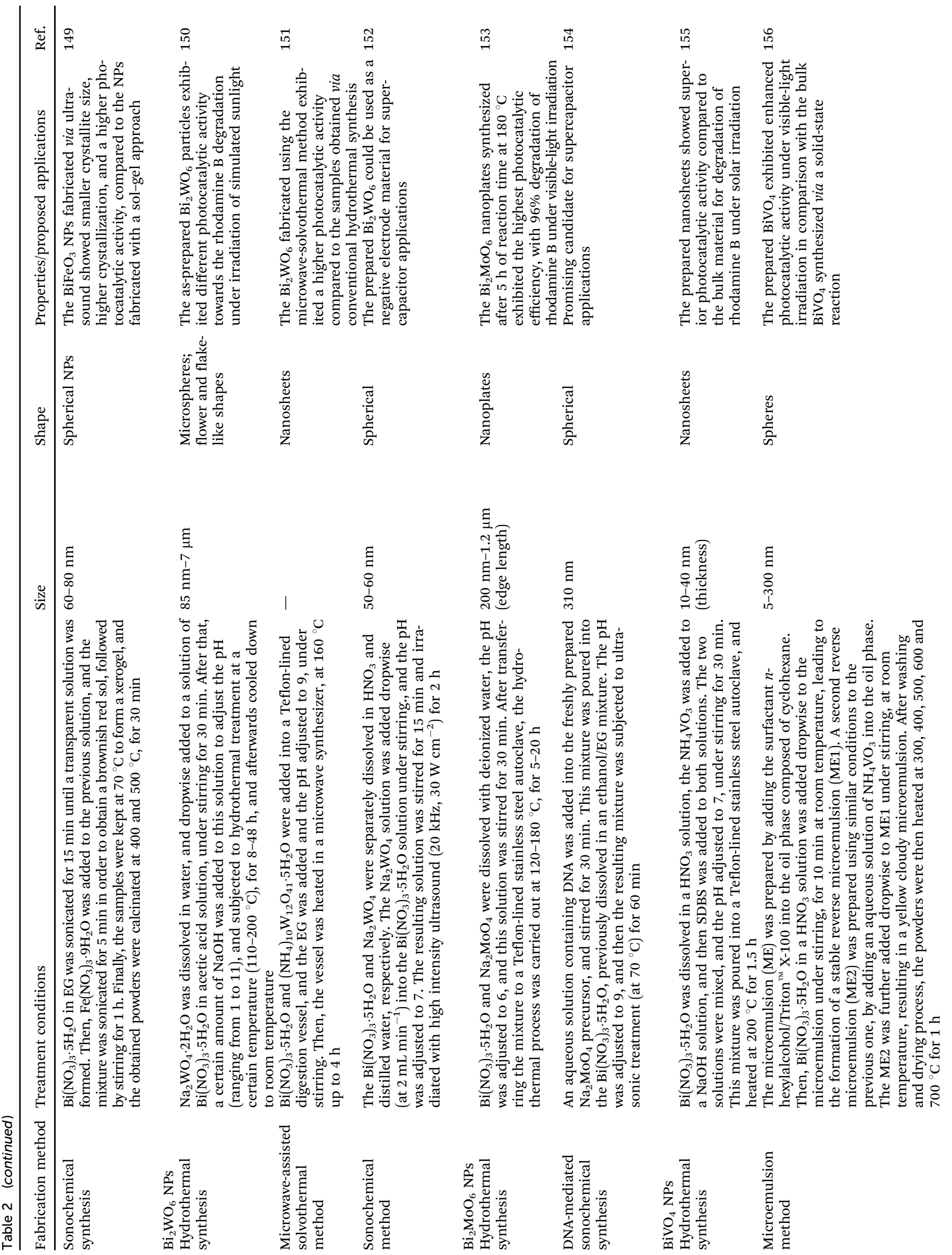




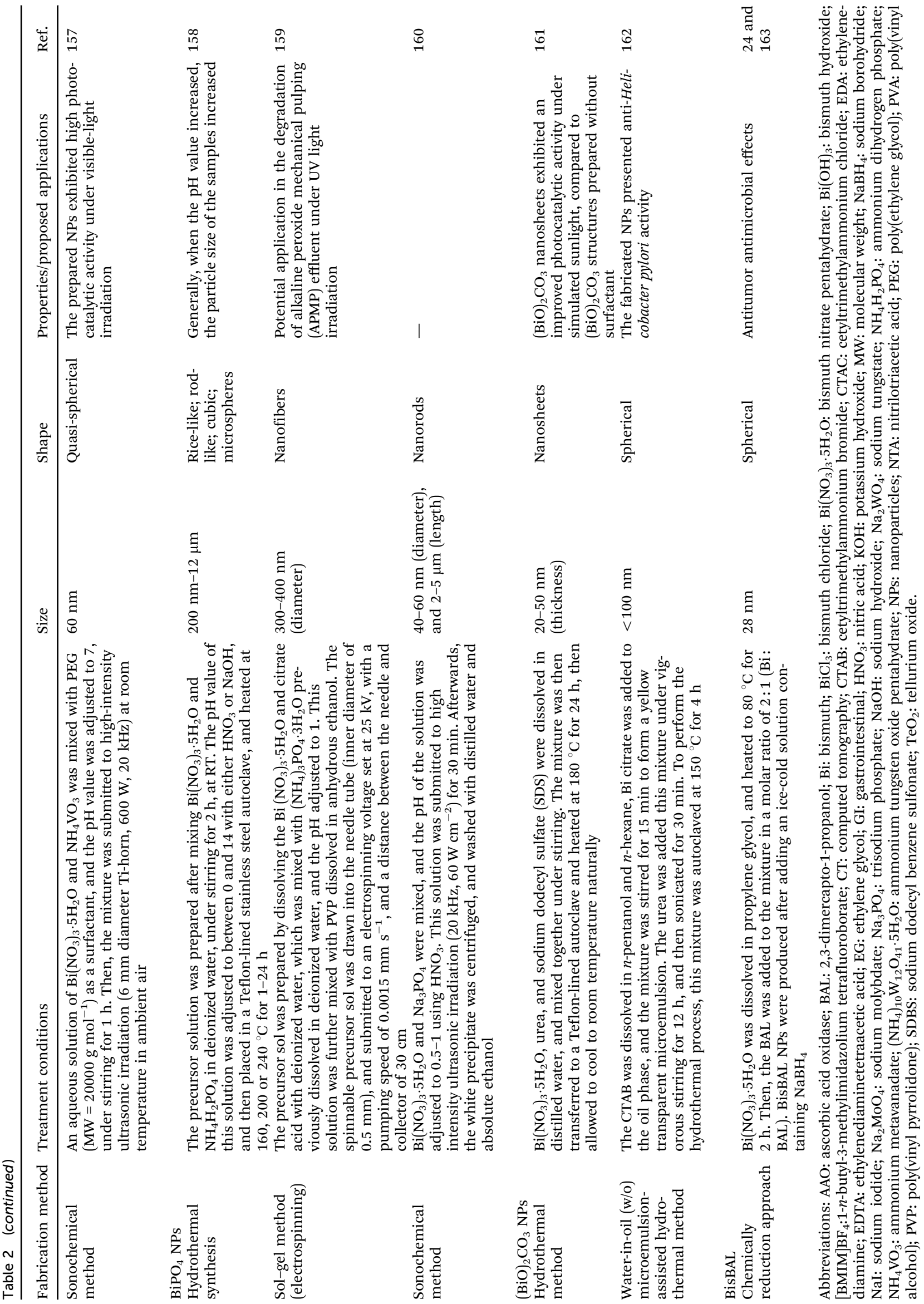



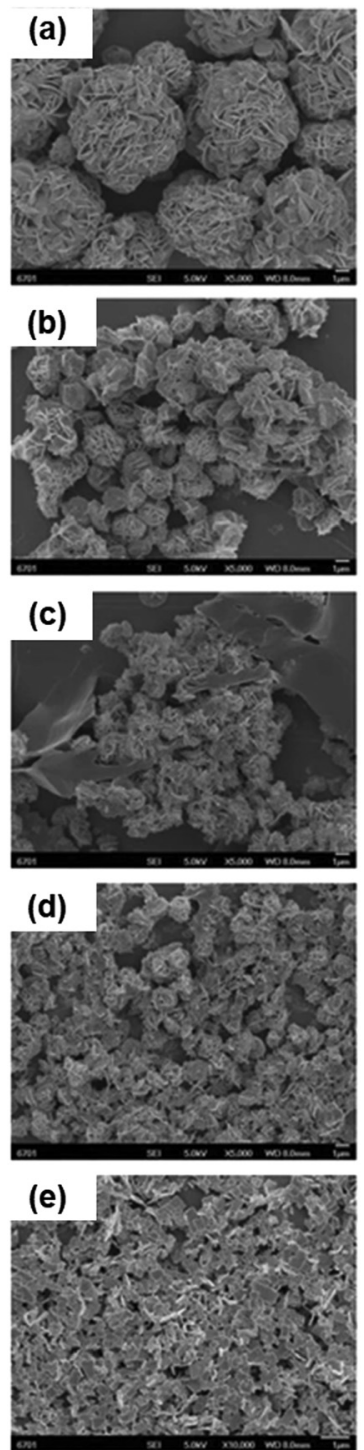
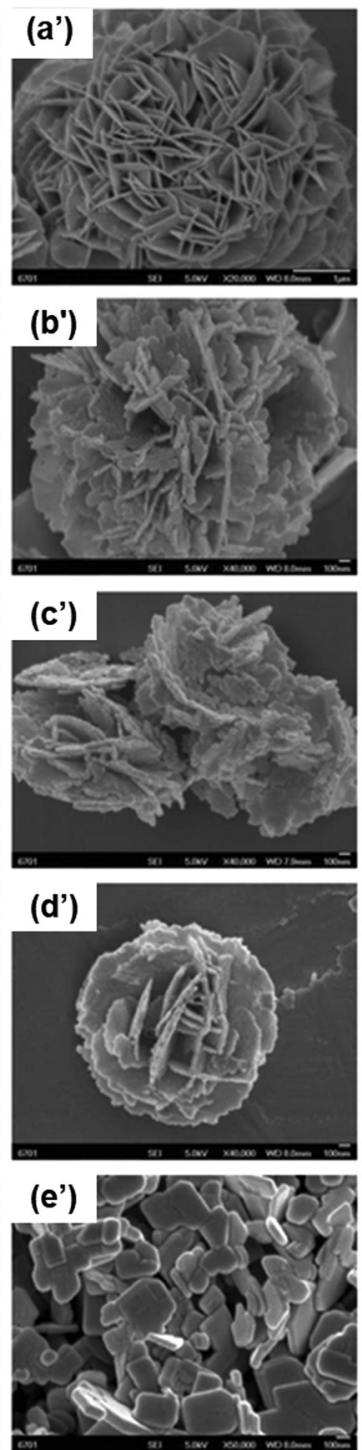
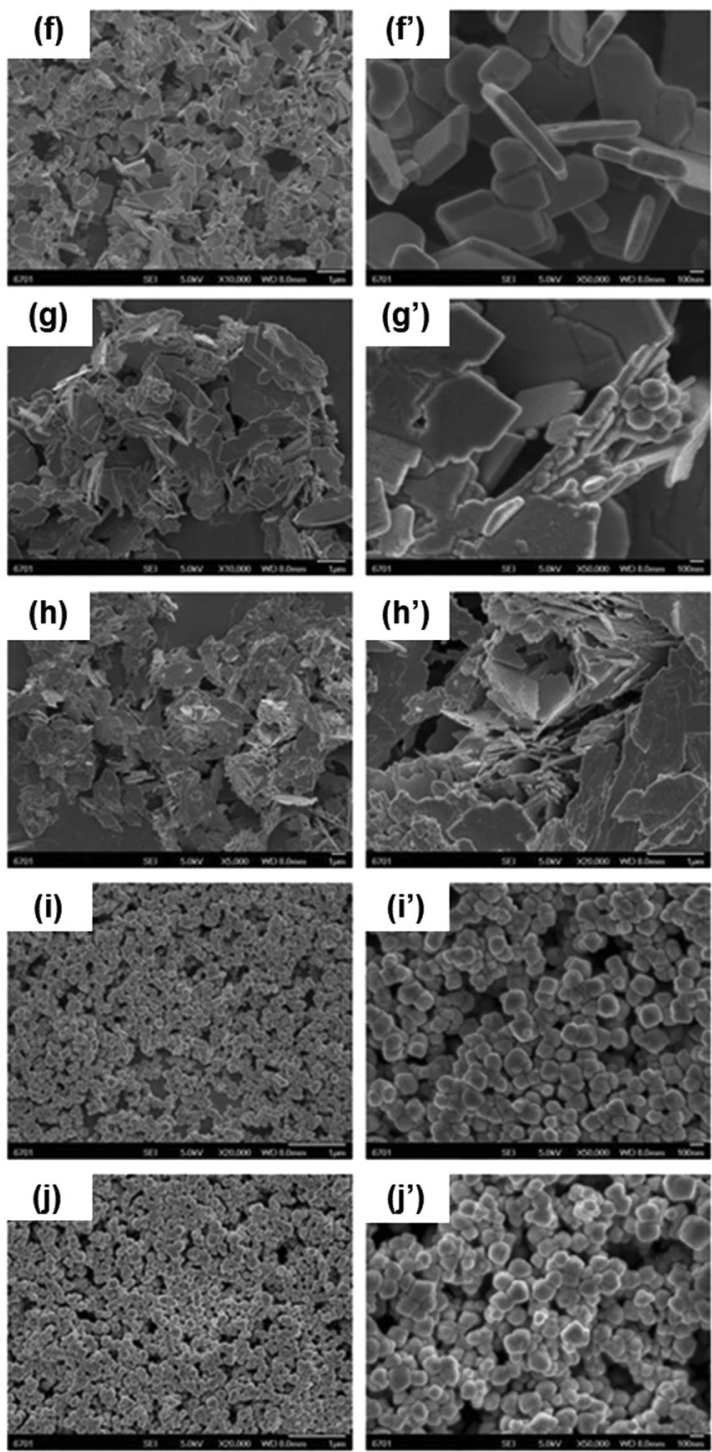

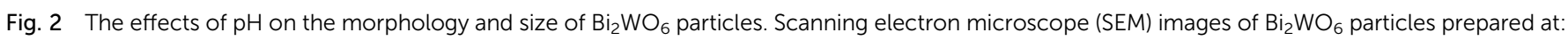

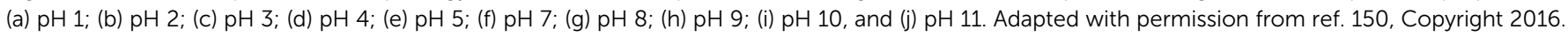

During the sol-gel synthesis of the BiNPs, the addition of chelating agents, such as acetic anhydride, acetic acid, citric acid, or tartaric acid, can determine the morphology and purity of the final product. Therefore, selection of the appropriated chelating agent is essential for the successful preparation of Bi nanostructures. ${ }^{65,175}$ Wang et al. synthesized pure $\mathrm{BiFeO}_{3}$ using a tartaric acid-assisted sol-gel method, with a particle size ranging from 60 to $90 \mathrm{~nm}$ and polyhedral morphology. ${ }^{175}$ After mixing $\mathrm{Bi}\left(\mathrm{NO}_{3}\right)_{3} \cdot 5 \mathrm{H}_{2} \mathrm{O}$ and $\mathrm{Fe}\left(\mathrm{NO}_{3}\right)_{3} \cdot 9 \mathrm{H}_{2} \mathrm{O}$ in dilute nitric acid, the tartaric acid in EG was successively added to the previous solution under stirring, and the resulting solution was kept at $140{ }^{\circ} \mathrm{C}$ until a dried gel was obtained. The gel was pretreated at $400{ }^{\circ} \mathrm{C}$ to remove organic compounds and $\mathrm{NO}_{3}{ }^{-}$, and then calcinated at $500{ }^{\circ} \mathrm{C}$ for $1 \mathrm{~h}$. After this process, pure $\mathrm{BiFeO}_{3}$ was obtained due to the formation of stable heterometallic polynuclear complex bonds between the metal ions and the tartaric acid. However, when citric acid was used as a chelating agent instead of tartaric acid, $\mathrm{Bi}_{2} \mathrm{Fe}_{4} \mathrm{O}_{9}$ and $\mathrm{Bi}_{25} \mathrm{FeO}_{40}$ impurities were observed, which can be attributed to the dimeric nature of the citrate complex.

Originally developed in the 1960s, the Pechini method is another common sol-gel synthesis technique used to prepare layered oxide perovskites, based on an esterification reaction between a carboxylic acid and an alcohol. ${ }^{176}$ Hasan et al. used a modified Pechini method to obtain $\mathrm{BiFeO}_{3}$ NPs with different morphologies through alteration of the annealing temperature. ${ }^{148}$ $\mathrm{Bi}\left(\mathrm{NO}_{3}\right)_{3} \cdot 5 \mathrm{H}_{2} \mathrm{O}$ and $\mathrm{Fe}\left(\mathrm{NO}_{3}\right)_{3} \cdot 9 \mathrm{H}_{2} \mathrm{O}$ were dissolved in water, citric acid was added as a chelating agent to complex the metal cations, and the solution was then stirred and heated at $70-75{ }^{\circ} \mathrm{C}$ for $3 \mathrm{~h}$. After that, EG was added as a polymerization agent, and the resultant solution was heated at $85-90{ }^{\circ} \mathrm{C}$ to initiate the polymerization reaction until a gel was formed. After drying the gel, the precursor xerogel was powdered and annealed at temperatures ranging from 400 to $800{ }^{\circ} \mathrm{C}$ for $2 \mathrm{~h}$ to obtain $\mathrm{BiFeO}_{3} \mathrm{NPs}$ with 

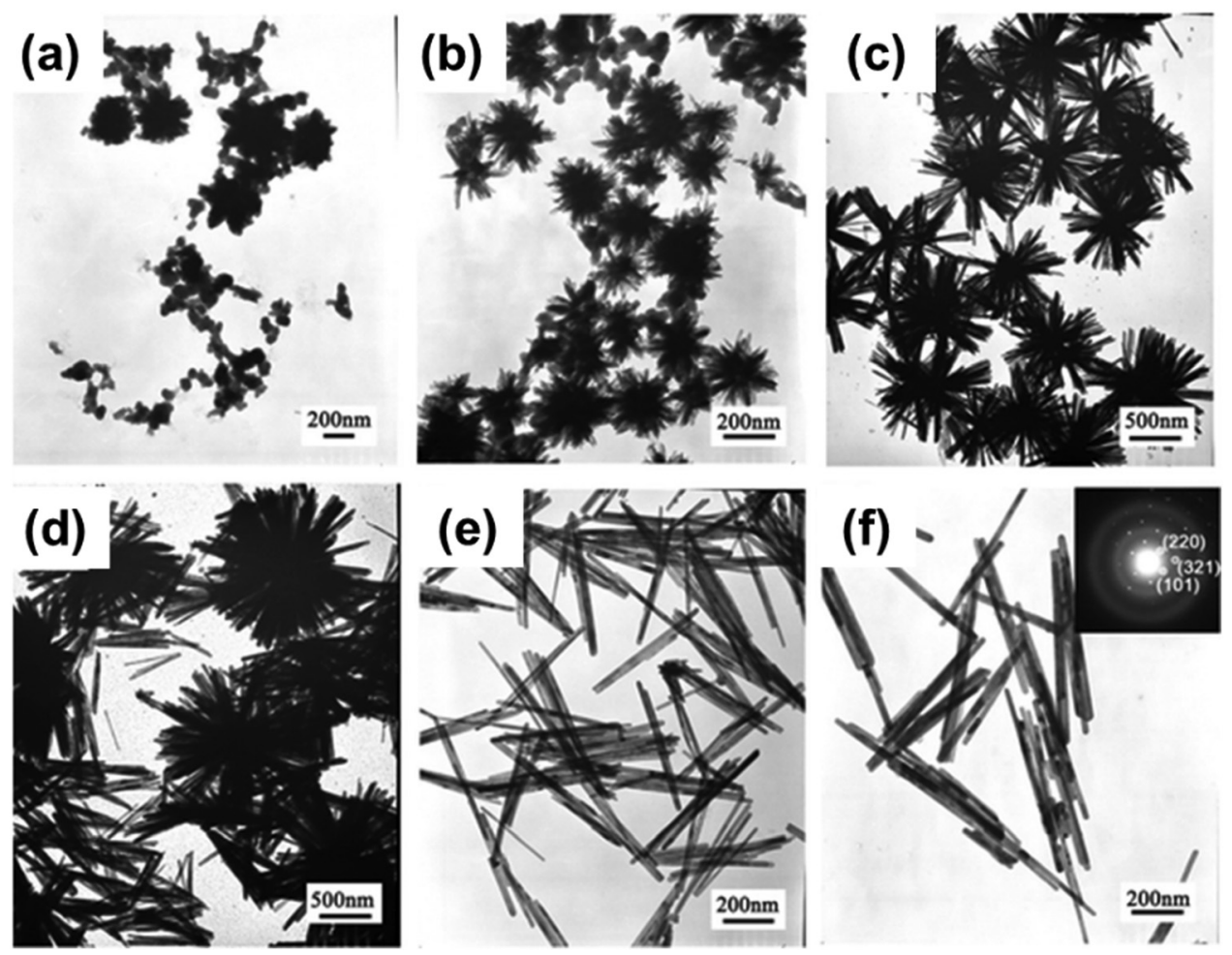

Fig. 3 Transmission electron microscope (TEM) images of $\mathrm{Bi}_{2} \mathrm{~S}_{3}$ nanostructures prepared after (a) $3 \mathrm{~s}$, (b) $5 \mathrm{~s}$, (c) $5 \mathrm{~min}$, (d) $30 \mathrm{~min}$, (e) $1 \mathrm{~h}$, and (f) $5 \mathrm{~h}$ of reaction time. Adapted with permission from ref. 134, Copyright 2010.

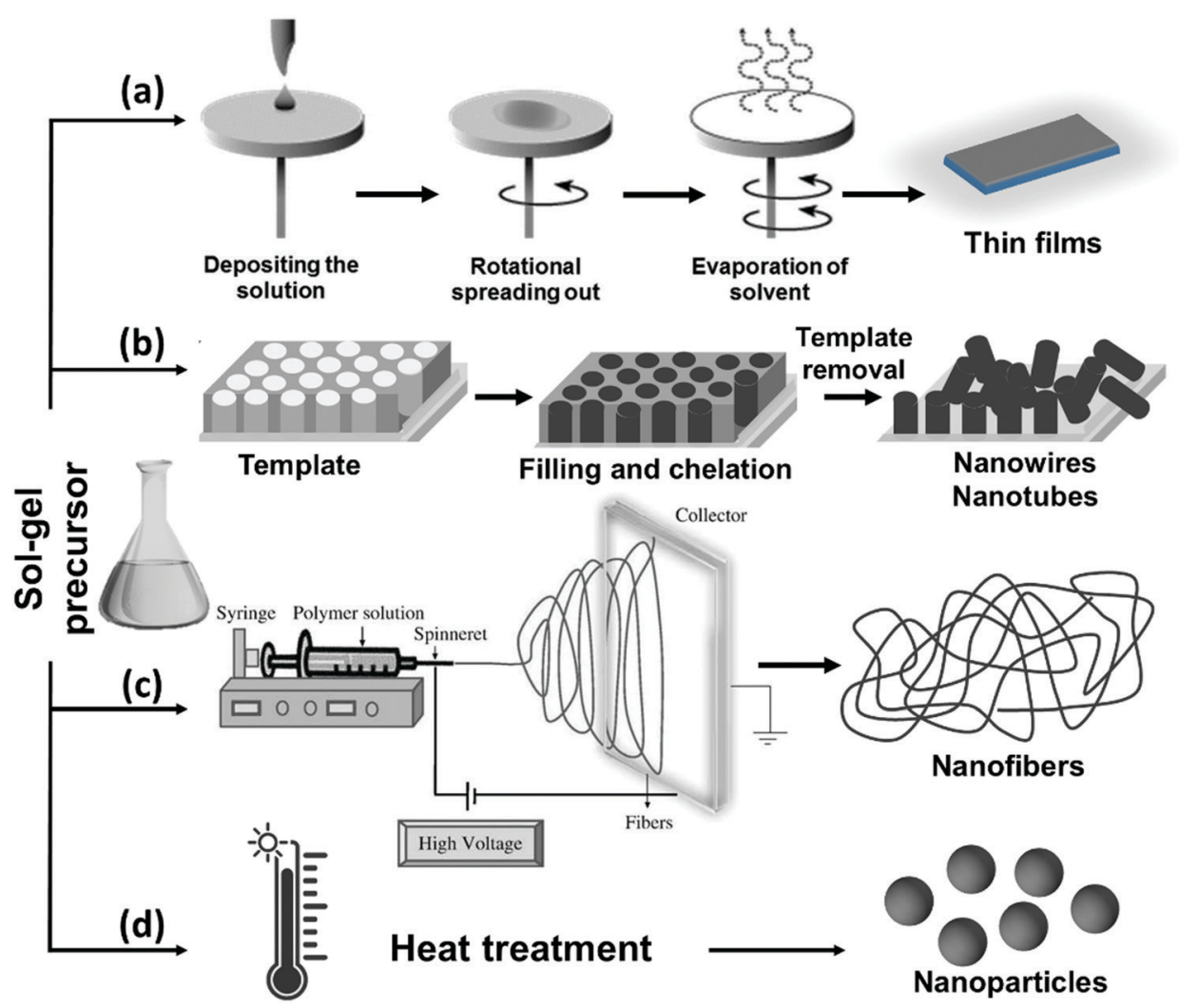

Fig. 4 A schematic diagram of the available sol-gel mediated approaches used for the fabrication of Bi-containing nanostructures. 
average sizes of 21-68 $\mathrm{nm}$. Spherical NPs were obtained when the powders were annealed up to $600{ }^{\circ} \mathrm{C}$, while stable cubic NPs were produced at an annealing temperature of $800{ }^{\circ} \mathrm{C}$.

As mentioned earlier, the sol-gel method can be combined with the electrospinning technique to obtain $\mathrm{BiFeO}_{3}$ nanofibers, as reported by Wang et al. ${ }^{177}$ For this purpose, the initial precursors $\mathrm{Bi}\left(\mathrm{NO}_{3}\right)_{3} \cdot 5 \mathrm{H}_{2} \mathrm{O}$ and $\mathrm{Fe}\left(\mathrm{NO}_{3}\right)_{3} \cdot 9 \mathrm{H}_{2} \mathrm{O}$ were dissolved in 2-methoxyethanol, and then glacial acetic acid and ethanolamine were added to adjust the viscosity and $\mathrm{pH}$-value of the solution under stirring. This solution was further mixed with PVP in dimethylformamide/acetone $(2: 1 \mathrm{v} / \mathrm{v})$. Afterwards, this precursor solution was loaded into a plastic syringe for the electrospinning process, using a direct current voltage of $13 \mathrm{kV}$. The collector (aluminum foil) was attached to the cathode, and the distance between the needle tip and the collector was $10 \mathrm{~cm}$. After evaporation of the solvent, a fibrous mat was formed on the collector, and the composite nanofibers were dried at $60{ }^{\circ} \mathrm{C}$ for $24 \mathrm{~h}$, followed by heating at $350{ }^{\circ} \mathrm{C}$ for $30 \mathrm{~min}$. Finally, thermal annealing was conducted under an argon (Ar) atmosphere at $550{ }^{\circ} \mathrm{C}$ for $2 \mathrm{~h}$ to allow the production of fibers with a rhombohedral distorted perovskite structure. These nanofibers, with a diameter of 220-480 nm, exhibited good magnetic response, which was ascribed to the nanometer size of the fibers, along with photocatalytic activity under ultraviolet (UV) and visible light irradiation.

\subsection{Microemulsion technique}

The microemulsion technique has attracted considerable attention in preparing colloidal metal nanostructures, due to the ability to control the different particle properties, such as size, geometry, morphology, homogeneity and surface area. ${ }^{178}$ Typically, in this technique, nanosized aqueous droplets are embedded with a surfactant, distributed in an organic phase (oil). The water phase of the microemulsion contains metal salts and acts as a nanoreactor for the synthesis of the particles, in which the shape and size of the particles can be tuned by adjusting the water: surfactant ratio. ${ }^{117,179}$ The microemulsion method allows the simultaneous synthesis and stabilization of NPs, in which the water nanodroplets limit the particle growth, and the presence of surfactants adsorbed on the particle surface reduces the surface tension and the possibility of agglomeration. The formation protocol of the $\mathrm{BiOI}-\mathrm{TiO}_{2}$ nanocomposite is schematically shown in Fig. 5a as a general microemulsion method to prepare BiNPs. ${ }^{180}$ The surfactant and co-surfactant can be chosen according to the nature of both the oil and water phases. By changing different parameters, such as the concentration of the different constituents and the nature of the dispersing phase in the microemulsion system, it is possible to control the particle morphology and size, and consequently, their properties and application. Nevertheless, the absence of a capping agent makes the NPs susceptible to agglomeration as well as surface oxidation, which is disadvantageous from materials handling and safety viewpoints. To cope with this drawback, Purkayastha et al. ${ }^{117}$ introduced a method using thioglycolic acid to cap $\mathrm{Bi}_{2} \mathrm{Te}_{2} \mathrm{NPs}$ using a microemulsion method (Fig. 5b). Two separate microemulsions were first formed from bis(2-ethylhexyl)sulfosuccinate (AOT)/isooctane mixtures, one containing orthotelluric acid and the second containing Bi chloride. Thioglycolic acid was then added to a bismuth chloride/AOT/isooctane solution to convert the transparent $\mathrm{Bi}$ chloride/AOT/isooctane solution into a yellow solution as a result of the $\mathrm{Bi}$ ionically bonding with the thioglycolic acid. Then, mixing of the two microemulsions was followed by adding hydrazine monohydrate to reduce the solution and form molecularly capped $\mathrm{Bi}_{2} \mathrm{Te}_{2} \mathrm{NPs}$, with size diameters ranging from 2.5 to $10 \mathrm{~nm}$.

\subsection{Chemical approaches}

Different chemical methodologies, including the chemical reduction method, as well as electrochemical and photochemical approaches,
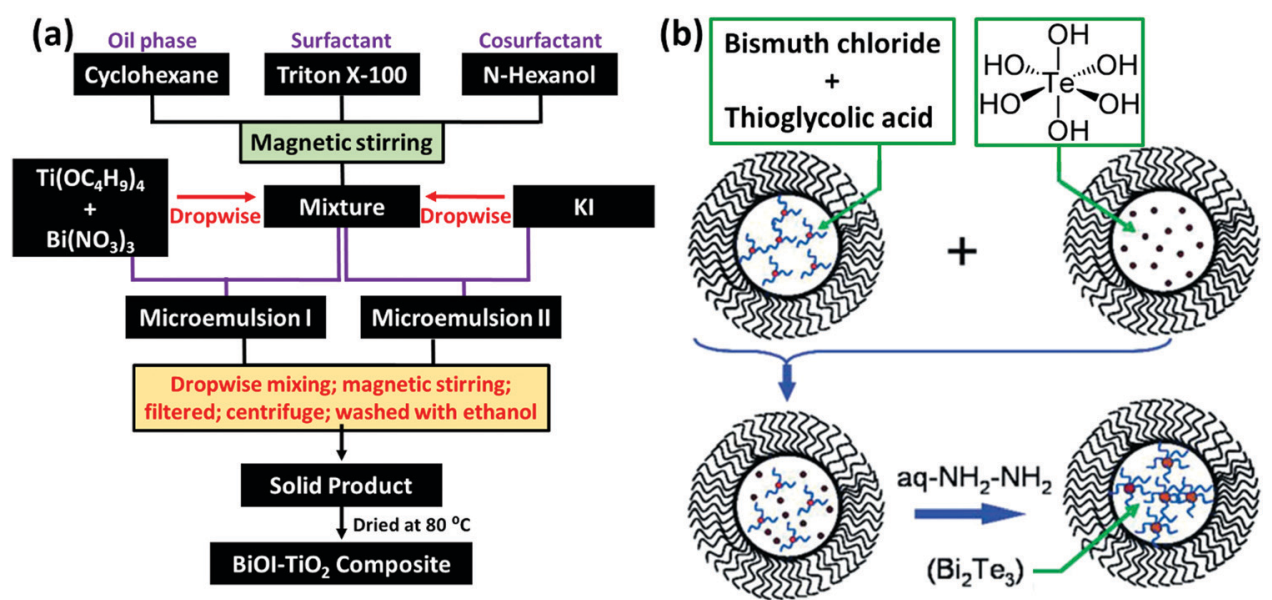

Fig. 5 (a) A flow chart showing the synthesis procedure of $\mathrm{BiOl}_{-} \mathrm{TiO}_{2}$ particles via the microemulsion method. Adapted with permission from ref. 180 , Copyright 2014. (b) The formation of $\mathrm{Bi}_{2} \mathrm{Te}_{2}$ NPs in water nanodroplets confined by bis(2-ethylhexyl)sulfosuccinate (AOT; black wiggles) in an isooctane solution. The microemulsion containing Bi ions (red dots) ligated with thioglycolic acid (blue squiggles) was mixed with another microemulsion containing orthotelluric acid (brown dots). The mixture was reduced using hydrazine monohydrate to produce thioglycolic acid capped $\mathrm{Bi}_{2} \mathrm{Te}_{2} \mathrm{NPs}_{\mathrm{S}}$ (brown spheres). Adapted and reprinted with permission from ref. 117, Copyright 2006. 

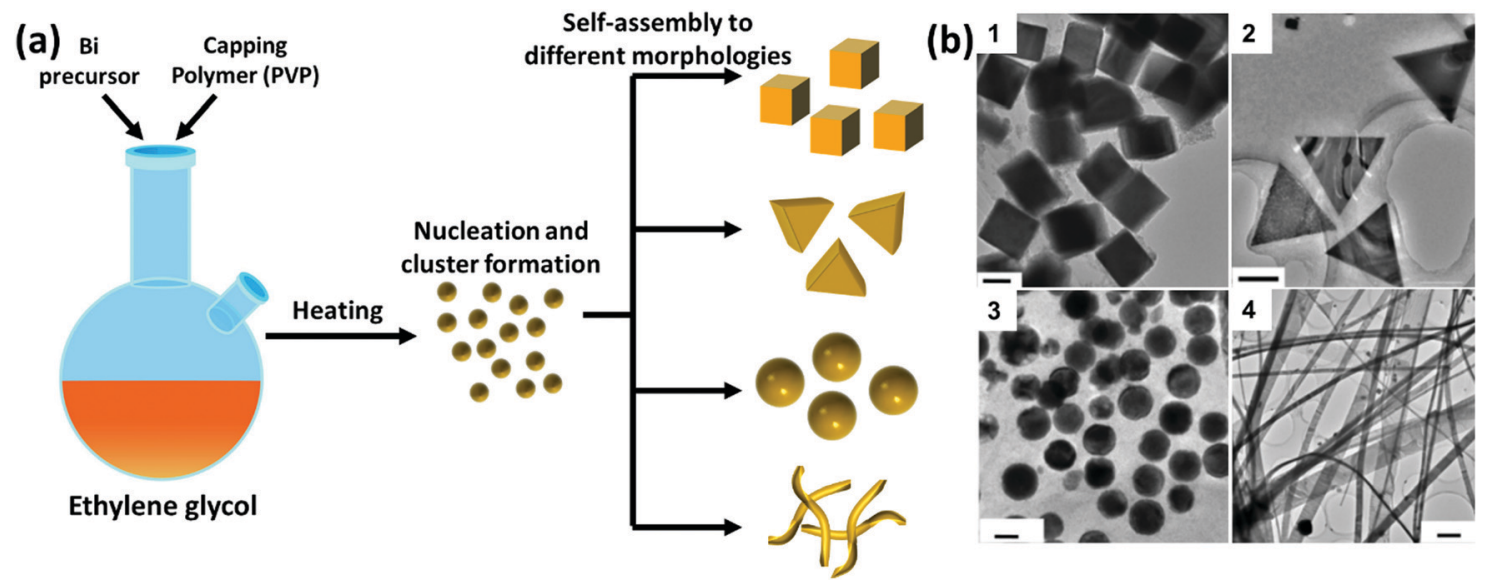

Fig. 6 (a) A schematic diagram of the polyol process for the synthesis of BiNPs and (b) representative TEM images of: (1) single-crystalline Bi nanocubes, prepared with a PVP : Bi molar ratio of 1.6 (scale bar $=50 \mathrm{~nm}$ ); (2) Bi triangular nanoplates, when the molar ratio of PVP: Bi was reduced to 0.8 (scale bar $=$ $200 \mathrm{~nm}$ ); (3) Bi nanospheres, after increasing the molar ratio of PVP: Bi to 5 (scale bar $=50 \mathrm{~nm}$ ); and (4) Bi nanobelts, prepared with a of PVP: Bi molar ratio of 1.6 in the presence of $\mathrm{Fe}^{3+}$ species (scale bar $=500 \mathrm{~nm}$ ). Adapted with permission from ref. 181, the American Chemical Society, Copyright 2006.

can be used to synthesize a variety of Bi structures. ${ }^{181-183}$ The polyol process is a soft chemical reduction technique used to fabricate a large variety of metallic NPs, presenting many advantages such as low cost, ease of use and facile scale-up. Polyols are diol, mainly 1,2-diols, such as ethylene glycol and its derivatives, including di-, tri-, tetra- and so on up to poly(ethylene glycol). The presence of several $\mathrm{OH}$ groups in polyols confers them with interesting properties, such as reduction and coordination capabilities, which contribute desirably to the synthesis of metal NPs. Typically, the polyol-mediated synthesis of metallic particles involves three main steps, including: (1) dissolving the metal precursor in an appropriate solvent, (2) reduction and nucleation of the monomer species, and (3) the growth and self-assembly of the nuclei/clusters to form metal particles. The reduction conditions are a very crucial step for controlling the size and morphology of the BiNPs during the polyol process, subsequently enabling the tailoring of the electronic, magnetic, optical, and catalytic properties of the NPs. ${ }^{184}$ For example, Wang et al. ${ }^{181}$ were able to control the size and shape of BiNPs using the polyol process (in the presence of ethylene glycol) by changing the ratio of $\mathrm{Bi}$ cations $\left(\mathrm{NaBiO}_{3}\right.$ as the source) to the capping polymer, PVP, at $200{ }^{\circ} \mathrm{C}$. As shown in Fig. 6, different sizes and morphologies of BiNPs were obtained by changing the PVP: Bi molar ratio. PVP acts as a shape controlling agent for the formation of Bi nanocubes and triangular nanoplates, and also as a stabilizing agent to prevent the Bi spherical particles from aggregating and forming big particles. Moreover, it was found that the addition of $\mathrm{Fe}^{3+}$ can reduce the nucleation rate of Bi NPs, allowing the NPs to align in the same direction and connect to each other to form wire-like structures. ${ }^{181}$

Strong reducing agents, such as alcohols, citrate ions, hydrazine or sodium borohydride $\left(\mathrm{NaBH}_{4}\right)$ have also been reported for the synthesis of BiNPs. ${ }^{184}$ Brown and Goforth ${ }^{120}$ synthesized BiNPs by reducing $\mathrm{Bi}\left(\mathrm{NO}_{3}\right)_{3} \cdot 5 \mathrm{H}_{2} \mathrm{O}$ in the presence of $\mathrm{NaBH}_{4}$, in a glycine and dextran containing solution at $\mathrm{pH}$ 9. Upon the addition of the reducing agent, the solution changed from a colorless to a black solution in a few minutes. The dextran was used as a surfactant to render high stability to BiNPs in aqueous solutions. The preparation of BiNPs in 1,2-propanediol, using a borane reducing agent, and glucose as a biocompatible surface stabilizer, has also been demonstrated by the same team. ${ }^{121}$ Borane was selected as a reducing agent due to its kinetically slower reactivity compared to $\mathrm{NaBH}_{4}$, providing better size and morphology control during the formation of BiNPs, and easy purification of the prepared BiNPs. The fraction of Bi atoms constituted $c a .4 \%$ of the particle volume ( $\sim 100000 \mathrm{Bi}$ atoms/NP) when the former method was used, while in the latter one, the BiNPs made up around $64 \%$ of the total NP volume, containing ca. 6 million Bi atoms per NP (Fig. 7). This higher Bi payload resulted in a substantial improvement in the sensitivity of the nanoplatform as an X-ray contrast agent for CT imaging. ${ }^{121}$

In addition to the above chemical approach, electrochemical and electrodeposition methods have been applied to fabricate BiNPs with different morphologies and Bi-containing films. ${ }^{182,185-187}$ Photochemical methodology is another convenient and environmentally friendly chemical approach suggested for the fabrication of BiNPs. For example, Zhao et al. reported the synthesis of $\mathrm{Bi}_{2} \mathrm{~S}_{3}$ nanoflowers on an alumina template using a photodeposition technique. ${ }^{188} \mathrm{Bi}\left(\mathrm{NO}_{3}\right)_{3}$ was used as a $\mathrm{Bi}$ source, and mixed with thioacetamide as a source of $\mathrm{S}^{2-}$, due to the ability of thioacetamide to decompose under UV irradiation and release $\mathrm{S}^{2-}$, in the presence of the complex agent nitrilotriacetic acid. Afterwards, an alumina template with pores of $100 \mathrm{~nm}$ in diameter was immersed in the previous solution, and the resulting solution was irradiated under UV light for $4 \mathrm{~h}$, using a $500 \mathrm{~W}$ high-pressure mercury lamp $(\lambda>290 \mathrm{~nm})$ as the source of UV irradiation. The presumed growth mechanisms of $\mathrm{Bi}_{2} \mathrm{~S}_{3}$ nanoflowers can be described as follows: (1) the solution was permeated through the pores of the alumina template, and the deposition was performed in the presence of UV light until the pores were totally filled; and (2) the formation of $\mathrm{Bi}_{2} \mathrm{~S}_{3}$ nanowires in the pores occurred, and the nanowires extended to the surface of the alumina template, and continued to grow until $\mathrm{Bi}_{2} \mathrm{~S}_{3}$ nanoflowers were formed. During this process, the irradiation time and $\mathrm{pH}$ of the solution were found to affect the 


\section{Fraction of Bi Atoms in NPs Prepared by Two Different Approaches}
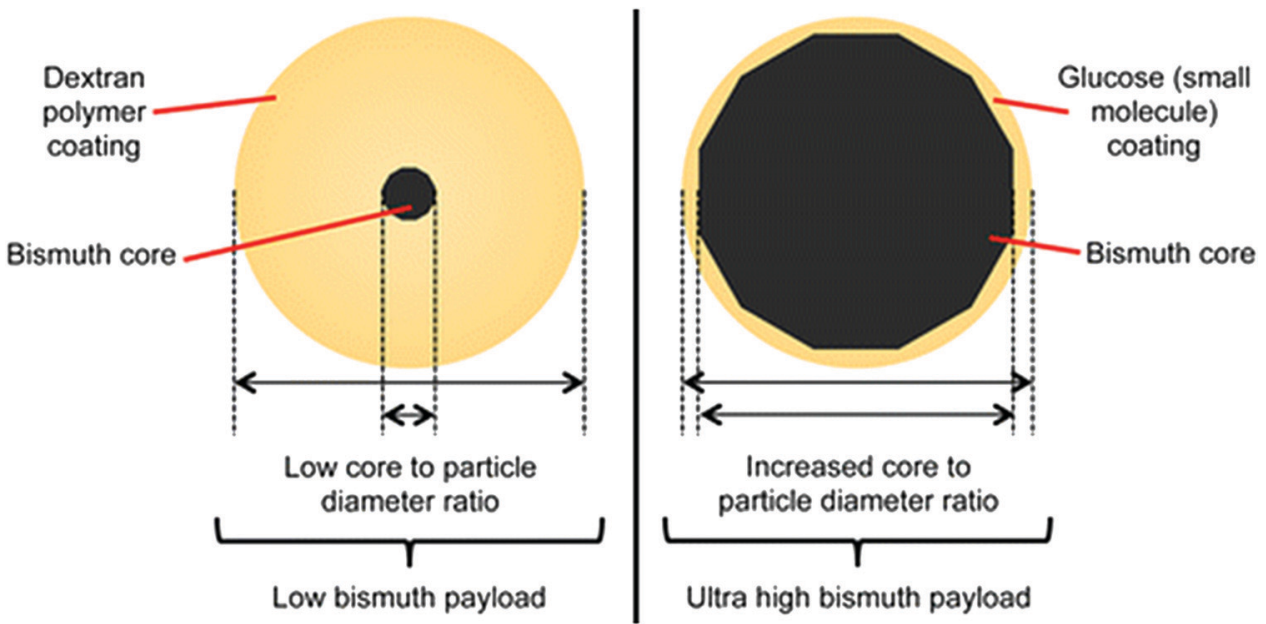

Fig. 7 A schematic comparison of the two BiNP formulations prepared by Brown et al. after the chemical reduction of $\mathrm{Bi}\left(\mathrm{NO}_{3}\right)_{3} \cdot 5 \mathrm{H}_{2} \mathrm{O} \cdot \mathrm{Reprinted}$ with permission from ref. 121, the American Chemical Society, Copyright 2014

morphology of the prepared $\mathrm{Bi}_{2} \mathrm{~S}_{3}$. When the reaction time was lower than $1 \mathrm{~h}$, the decomposition reaction of the Bi-S did not occur and $\mathrm{Bi}_{2} \mathrm{~S}_{3}$ was not formed. Moreover, a reaction time of $4 \mathrm{~h}$ was required to form nanoflowers on the alumina template, after a complete filling of the alumina template. Additionally, the $\mathrm{pH}$ of the solution was found to affect the formation of the $\mathrm{Bi}_{2} \mathrm{~S}_{3}$ nanoflowers.

\subsection{Microwave irradiation method}

The microwave irradiation synthesis has been employed in the fabrication of different inorganic materials, including BiNPs, because it is generally quite simple, with a short reaction time, and very energy efficient, compared to the abovementioned methods. ${ }^{189}$ Additionally, this green synthesis method allows the production of high purity particles with different morphologies, with a small average size and narrow size distribution. ${ }^{65,190}$ For example, Liao et al. ${ }^{190}$ fabricated $\mathrm{Bi}_{2} \mathrm{~S}_{3}$ nanorods after reacting an aqueous formaldehyde solution, containing $\mathrm{Bi}\left(\mathrm{NO}_{3}\right)_{3} \cdot 5 \mathrm{H}_{2} \mathrm{O}$ and thiourea, in a microwave refluxing system for $20 \mathrm{~min}$, obtaining nanorods with a diameter of around $10 \mathrm{~nm}$ and a length of up to $300 \mathrm{~nm}$. Formaldehyde solution was selected as a solvent, because it led to the formation of pure and uniform nanorods. Water and ethanol were also tested as solvents, resulting in the formation of aggregated short rods. Additionally, the formation of uniform and small-sized nanorods occurred when thiourea was used as a source of sulfur. When thioacetamide was employed as the sulfur source, the prepared $\mathrm{Bi}_{2} \mathrm{~S}_{3}$ nanorods were found to be thicker and longer (average diameter of $50 \mathrm{~nm}$ and length up to $1-2 \mu \mathrm{m}$ ).

In the microwave irradiation method, ionic liquids are excellent media for absorbing microwaves, leading to a fast heating rate. It was demonstrated that when the ionic liquid 1-n-butyl-3methylimidazolium tetrafluoroborate $\left([\mathrm{BMIM}] \mathrm{BF}_{4}\right)$ was used as a solvent in the microwave irradiation process, the formation of pure hexagonal-shaped $\mathrm{Bi}_{2} \mathrm{Se}_{3}$ nanosheets was favored. ${ }^{131}$
However, when EG was used as the solvent in the absence of $[\mathrm{BMIM}] \mathrm{BF}_{4}$, irregular-shaped nanosheets were obtained, suggesting that the presence of the ionic liquid influences the morphology of the BiNPs. Therefore, changing the solvent and manipulating the ratio of the solvent Bi source is very crucial in the optimization of the microwave irradiation mediated synthesis of BiNPs. In this context, $\mathrm{Li}$ et $a .^{32}$ were able to fabricate different $\mathrm{BiPO}_{4}$ nanostructures (Fig. 8), which exhibited
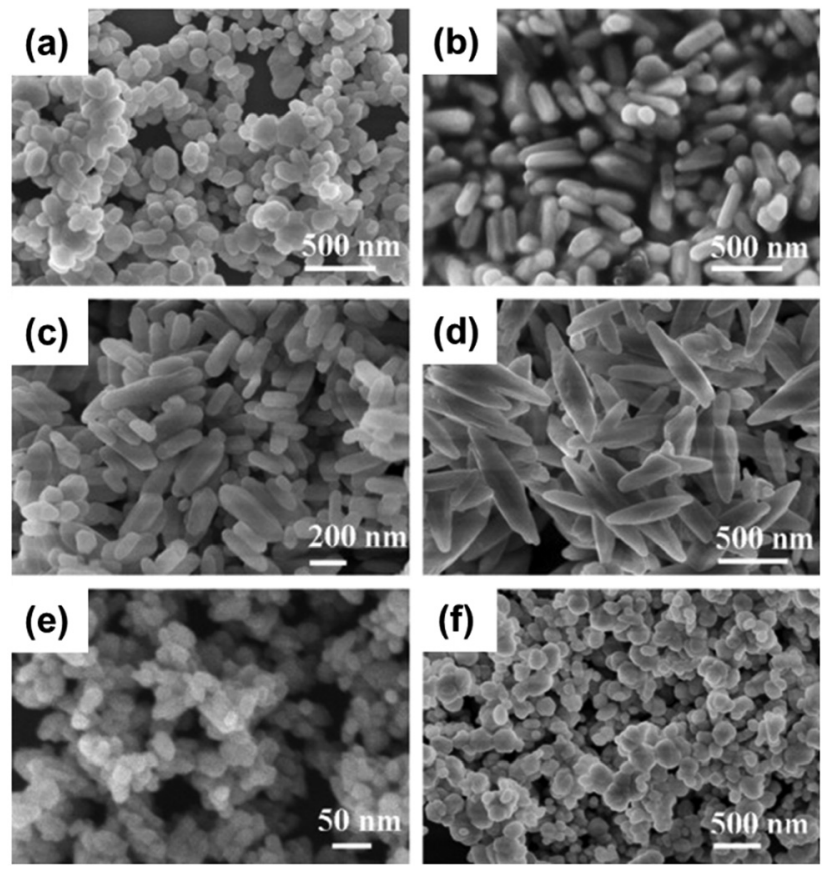

Fig. 8 SEM images of the different $\mathrm{BiPO}_{4}$ nanostructures fabricated using the following solvents with different viscosities: (a) glycerol/water (ratio 1:6); (b) glycerol/water (ratio 1: 9); (c) EG/water (ratio 2:1); (d) EG/water (ratio 1:6); (e) diethylene glycol; and (f) $0.6 \mathrm{M}$ mannitol. Reprinted with permission from ref. 32, the American Chemical Society, Copyright 2011. 
morphology-dependent photocatalytic behavior. For that, $\mathrm{Bi}\left(\mathrm{NO}_{3}\right)_{3} \cdot 5 \mathrm{H}_{2} \mathrm{O}$ was dissolved with different solvent combinations, such as glycerol/water or EG/water in different ratios, pure diethylene glycol, or $0.6 \mathrm{M}$ mannitol, and then mixed with $\mathrm{NaH}_{2} \mathrm{PO}_{4} \cdot 2 \mathrm{H}_{2} \mathrm{O}$. The resulting mixture was further heated using an $800 \mathrm{~W}$ microwave reactor for $15 \mathrm{~min}$, under continuous and vigorous stirring. The viscosity of the solvents played an important role in the morphology and size of the $\mathrm{BiPO}_{4}$ nanostructures, in which high viscosity of the pure diethylene glycol, for example, favored the formation of $\mathrm{BiPO}_{4}$ NPs (Fig. 8e), while low viscosity of the EG/water mixtures was crucial for the formation of $1 \mathrm{D} \mathrm{BiPO}_{4}$ nanostructures, such as short nanorods or rice-like NPs (Fig. 8c and d).

\subsection{Sonochemical- and laser-mediated approaches for the synthesis of BiNPs}

The application of high-intensity ultrasound is another facile and versatile method that can be applied for the fabrication of several types of nanostructured materials. ${ }^{191}$ Generally, the so-called sonochemical approach takes advantage of the extreme conditions induced by ultrasound, which creates unique hot spots that are high in temperature and pressure $(>5000 \mathrm{~K}$, and $>1000 \mathrm{~atm}$ ), and the cooling rate is over $1010 \mathrm{~K} \mathrm{~s}^{-1}$ when these bubbles implode. ${ }^{192,193}$ This technique relies on two physical phenomena associated with ultrasound: (1) acoustic cavitation, which involves the formation, growth and implosive collapse of bubbles in liquid; and (2) nebulization, which involves the creation of mist from ultrasound passing through a liquid and impinging on a liquid-gas interface, being the basis for ultrasonic spray pyrolysis. ${ }^{191}$ The main advantages of this method compared to other conventional fabrication processes are the rapid reaction rate and controllable reaction conditions, as well as the ability to form NPs with uniform shapes, narrow size distributions, and high purities. ${ }^{115}$ Wang et al. ${ }^{115}$ demonstrated that ultrasound irradiation is favorable for the fabrication of uniform-shaped $\mathrm{Bi}_{2} \mathrm{~S}_{3}$ nanorods, with a diameter ranging from 20 to $30 \mathrm{~nm}$, and a length of $c a .200-250 \mathrm{~nm}$. For that, $\mathrm{Bi}\left(\mathrm{NO}_{3}\right)_{3} \cdot 5 \mathrm{H}_{2} \mathrm{O}$ and sodium thiosulfate $\left(\mathrm{Na}_{2} \mathrm{~S}_{2} \mathrm{O}_{3}\right)$ were dissolved with distilled water, in the presence of triethanolamine as a complexing agent. This mixture was then subjected to high-intensity ultrasound irradiation, using a high-intensity ultrasonic probe $\left(0.6 \mathrm{~cm}\right.$ diameter; $\left.20 \mathrm{kHz}, 60 \mathrm{~W} \mathrm{~cm}^{-2}\right)$ immersed in the solution, for $2 \mathrm{~h}$, at room temperature. However, when all the reaction conditions were kept the same but thioacetamide was used as a sulfur source, shorter and thinner $\mathrm{Bi}_{2} \mathrm{~S}_{3}$ nanorods were obtained, which might be due to the higher nucleation rate of $\mathrm{Bi}_{2} \mathrm{~S}_{3}$ compared to the one observed when $\mathrm{Na}_{2} \mathrm{~S}_{2} \mathrm{O}_{3}$ is the sulfur source. Furthermore, changing the complexing agents also affects both the nucleation and growth rates of $\mathrm{Bi}_{2} \mathrm{~S}_{3}$, leading to the formation of nanorods with different lengths and diameters. For example, when EDTA was employed as a complexing agent, the $\mathrm{Bi}_{2} \mathrm{~S}_{3}$ nanorods presented an average diameter of $15 \mathrm{~nm}$ and length of $100 \mathrm{~nm}$; while using sodium tartrate resulted in shorter and more aggregated $\mathrm{Bi}_{2} \mathrm{~S}_{3}$ nanorods with mean dimensions of $20 \times 60 \mathrm{~nm}$. Additionally, adding $20 \% N, N$-dimethylformamide
(DMF) to the water solution increased the yield of the reaction, and also decreased the dimensions of the $\mathrm{Bi}_{2} \mathrm{~S}_{3}$ nanorods to $6 \times 30 \mathrm{~nm}$, due to the faster nucleation.

The presence of other surfactants in the precursor solution can also affect the size of the Bi nanostructures prepared via the sonochemical approach. In a study conducted by Zhang et al., $\mathrm{Bi}_{2} \mathrm{O}_{3}$ nanocrystals of different sizes were prepared after adding PVP to the initial solution composed of $\mathrm{Bi}\left(\mathrm{NO}_{3}\right)_{3} \cdot 5 \mathrm{H}_{2} \mathrm{O}$ in nitric acid. ${ }^{194}$ This solution was slowly dropped into a $\mathrm{NaOH}$ aqueous solution ( $\mathrm{pH}=11)$ under constant stirring, and the mixture was then irradiated with high-intensity ultrasound $(600 \mathrm{~W}, 20 \mathrm{kHz})$ for $75 \mathrm{~min}$. The particles prepared without PVP presented diameters of $450-700 \mathrm{~nm}$ and lengths of $1-2.5 \mathrm{~mm}$, and were rod-like in shape; while in the presence of $\mathrm{PVP}$, the $\mathrm{Bi}_{2} \mathrm{O}_{3}$ morphology changed to granular grains, with a particle size ranging from 40 to $100 \mathrm{~nm}$. The $\mathrm{Bi}^{3+}$ ions might coordinate with PVP, which decreases the $\mathrm{Bi}^{3+}$ concentration, and then the reaction between the $\mathrm{Bi}^{3+}$ ions and $\mathrm{OH}^{-}$is partially inhibited, leading to the formation of smaller sized crystals.

Bi nanostructures can also be fabricated using a laserassisted approach, in which a laser beam is used to vaporize or ablate $\mathrm{Bi}$, and later the products are collected on a substrate or then dispersed in a liquid. ${ }^{111}$ Different power sources have been explored to synthesize Bi-based nanocomposites via a laser-mediated approach. In the case of the pulsed laser radiation in liquid technique, the size distribution of the pure BiNPs can be tuned by changing the different parameters of the laser, including the pulse energy, pulse duration, wavelength, focalization spot, and repetition rate. ${ }^{124}$ Additionally, the lasermediated synthesis of pure BiNPs in aqueous solutions at different $\mathrm{pH}$ values, such as water $(\mathrm{pH} 7)$, water + sodium hydroxide (pH 9.7), or water + hydrochloric acid (pH 2.7) can lead to the formation of different morphologies, such as spherical, core-shell NPs, and hollow spheres, respectively. ${ }^{195}$

\subsection{Bismuth-based nanocomposites}

In addition to all of the techniques mentioned above, a diverse range of Bi-based nanocomposites have been prepared in order to improve the performance of BiNPs in different biomedical applications, such as antibacterial materials, drug delivery, cancer therapy, imaging, electrochemical sensors, and tissue engineering. ${ }^{123,196}$ The combination of BiNPs with several types of materials has been proposed, including with carbon-based materials, bioactive glasses, inorganic NPs, and polymers (e.g., PVP and PEG). ${ }^{48,52,123,196-199}$ For example, the construction of Bi heterojunctions with $\mathrm{TiO}_{2}$ or other materials, ${ }^{200-203}$ doping of various materials on BiNPs, ${ }^{204-207}$ as well as coating with various polymers, have been greatly focused on in the fabrication of Bi-based nanocomposites. Among all of these, the in vivo application of polymer camouflaged Bi-based nanocomposites has been highly studied as they show better stability in aqueous solutions and prolonged circulation time in the bloodstream. ${ }^{198,208-210}$ Surface PEGylation, ${ }^{211}$ BiNP encapsulation into a polymeric $\mathrm{NP},{ }^{212}$ layer-by-layer polymeric coating, ${ }^{213}$ and surface crosslinking of polymers have been proposed ${ }^{214}$ as the main strategies to hide BiNPs within a polymeric network. For example, 


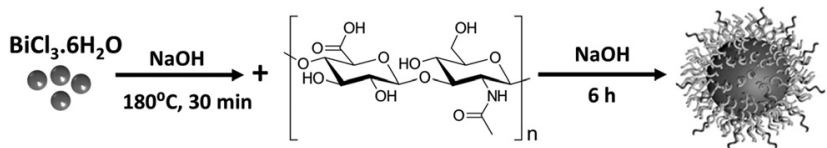

Fig. 9 A schematic diagram showing the procedure for fabricating $\mathrm{HA}-\mathrm{Bi}_{2} \mathrm{O}_{3} \mathrm{NPs}$.

hyaluronic acid (HA) modified $\mathrm{Bi}_{2} \mathrm{O}_{3}$ NPs were prepared using a slightly modified procedure of the "polyol" method. ${ }^{214}$ As shown in Fig. 9, a transparent viscous solution of $\mathrm{BiCl}_{3}$ was first prepared in diethylene glycol (DEG). The solution was then heated in a silicon oil bath at $140-160{ }^{\circ} \mathrm{C}$ for $1 \mathrm{~h}$ before adding $7.5 \mathrm{mmol}$ of $\mathrm{NaOH}$ dissolved in $30 \mathrm{~mL}$ of DEG. Next, dissolved reactants were heated at $180{ }^{\circ} \mathrm{C}$ for $30 \mathrm{~min}$ under vigorous stirring to obtain BiNPs, which were then further modified with HA through stirring in a basic solution of HA at room temperature for $6 \mathrm{~h}$. In the next sections, Bi-based nanocomposites are discussed more in detail, while describing their fabrication approach along with highlighting their biomedical applications.

\section{Biosafety and biodistribution of BiNPs}

With all the advances in NP-based formulations, the potential toxicity has remained a challenging hurdle for their bench-tobedside movement. This is mainly due to the high chemical reactivity and biological activity of the NPs as a result of their ultra-small size, as well as their intimate contact with cell membrane, internal organelles of cells, proteins and DNA. ${ }^{215,216}$ As one of the least toxic heavy metals, Bi has been clinically used for many years to treat different diseases, such as peptic ulcers, diarrhea, syphilis, severe acute respiratory syndrome (SARS), and gastrointestinal disorders. ${ }^{217-220}$ The most common conventional pharmaceutical formulations of $\mathrm{Bi}$, including bismuth subcitrate (De-Nol), bismuth subcitrate potassium (Pylera), bismuth subsalicylate (Helidac), and ranitidine bismuth citrate (Pylorid, Tritec) have demonstrated very high safety. Nevertheless, there are serious concerns with the in vivo toxicity of BiNPs, since comprehensive compatibility information on them is missing and current studies do not present the mechanisms behind the possible cytotoxicity of these NPs at high concentrations. ${ }^{221}$ Therefore, systematic studies on the generation of reactive oxygen species (ROS), mitochondrial toxicity, cell membrane damage, and DNA fragmentation, as the main mechanisms of NP-induced cellular damage, are needed after cell exposure to various types of BiNPs.

It is speculated that, similar to many other types of nanomaterials, BiNPs usually possess dose-dependent adverse biological effects and their cytotoxicity is largely dependent on particle diameter, charge, and surface modifications. For example, the coating of $\mathrm{Bi}_{2} \mathrm{~S}_{3}$ NPs by PVP, with the aim of improving their biological stability, blood circulation time, and safety, was found to keep HeLa adenocarcinoma cells alive at high concentrations in a dose-dependent manner (Fig. 10a). ${ }^{222}$ PVP coating has also been shown to increase the dispersity of
NPs in aqueous medium without any detectable agglomeration, resulting in slower elimination from the bodies of rats. The leaching of free $\mathrm{Bi}^{3+}$ ions, which is one of the main mechanisms of toxicity for BiNPs, is prohibited in the bloodstream. ${ }^{223}$ In addition, long-term histological screening of the major organs, including the heart, liver, spleen, and kidneys, showed no tissue damage associated with the single-dose administration of $\mathrm{PVP}-\mathrm{Bi}_{2} \mathrm{~S}_{3}$. PEG coating BiNPs can also reduce damage to cells. $^{208,224}$ PEG is a superior choice to modify the surface of NPs with respect to the following benefits, including the "stealth" effect of PEG on improved blood compatibility, as well as reduced protein corona formation and opsonization, resulting in prolonged blood circulation. ${ }^{225}$ Nevertheless, in contrast to the general assumption that PEG-conjugated NPs lack antigenicity and immunogenicity, anti-PEG immunoglobulin M (IgM) may be elicited after the introduction of PEGylated NPs within the body. ${ }^{226,227}$ For active suppression of immune attack and the prolonging of blood circulation, the biomimetic coating of NPs by with the membrane of red blood cells (RBCs) has been gaining increased attention. ${ }^{228} \mathrm{RBC}$ membrane has biologically friendly glycans, proteins and acidic sialyl moieties, which endow them with high biocompatibility, biodegradability, and non-immunogenicity. As shown in Fig. 10b1, longer retention time in blood circulation was detected for the RBC membrane-coated BiNPs (RBC-BiNPs) in comparison to their bare counterparts, mainly due to the reduced NP uptake by macrophages. $^{229}$ In this study, folate modified RBC (F-RBC)coated BiNPs showed a similar blood circulation trend as the ones camouflaged just using RBC membrane, demonstrating the negligible effect of folate on the circulation time. Compared to the bare NPs, prolonged circulation of RBC-BiNPs resulted in improved tumor accumulation via passive targeting $24 \mathrm{~h}$ postinjection. Folate conjugation can further enhance the cellular uptake of NPs by cancer tissue through combined passive and active targeting as a result of the presence of the RBC cell membrane and folate ligand, respectively (Fig. 10b2). Considering the high accumulation of the NPs in the liver and spleen, these two are considered as dominant organs in the metabolism of NPs, apart from their surface properties and coating functionality. ${ }^{230,231}$ High safety, reduced clearance by macrophages, and enhanced accumulation in the tumor have also been observed for mesoporous silica-coated $\mathrm{Bi}_{2} \mathrm{~S}_{3}$ nanorods (BMSNR) encapsulated within platelet membrane (PM; Fig. 10c1). Inspired by the in vivo stability of autologous molecules, elimination of the exogenous $\mathrm{Bi}_{2} \mathrm{~S}_{3}$ nanorods by macrophages was hindered after loading within the PM. Meanwhile, binding affinity of the PM to tumor cells resulted in active targeting of the 4T1 mammary gland breast cancer cells in vivo, as shown in Fig. 10c2 and c3. ${ }^{232}$ In contrast to the RBC cell membrane coating, the active targeting observed for the latter example can be attributed to the high affinity of the P-selectin on the surface of the PM for cancer cells. $^{233-235}$ Increased blood circulation time through polymeric or cell membrane coating may lead to concerns regarding the hemotoxicity of the BiNPs. Various reports have recently revealed the desirable hemocompatibility of BiNPs, showing a RBC lysing effect of lower than $5 \%,{ }^{48,213,236,237}$ which 
<smiles>CC(N)=S</smiles><smiles>CCCC(C)(C)C(=O)OC(C)(C)Br</smiles>
$\frac{\text { ODE and OA }}{1 \mathrm{~min}, 105^{\circ} \mathrm{C}}$ $=$ OA coated $\mathrm{Bi} 2 \mathrm{~S} 3$ nanodots

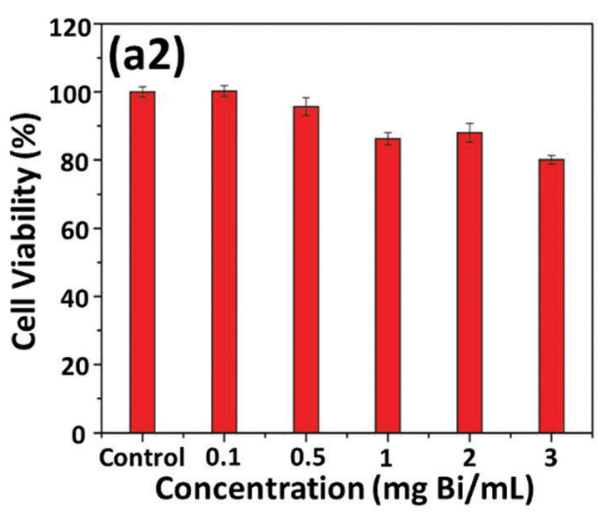

(a3) Spleen

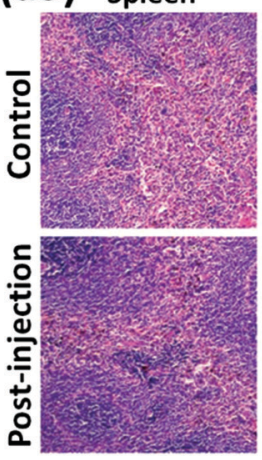

Kidney

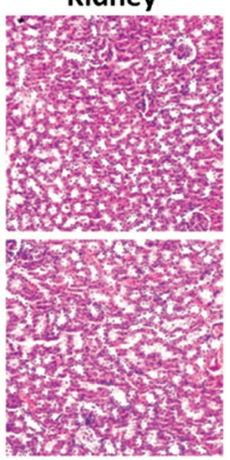

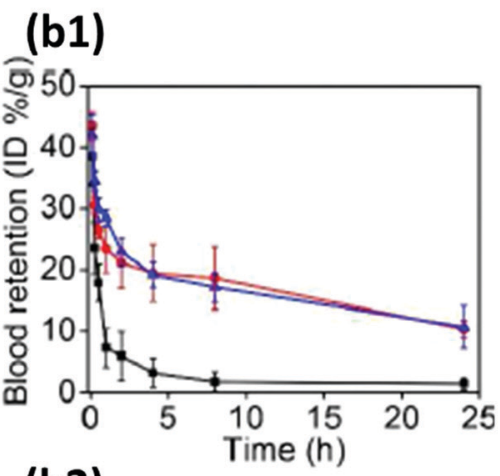

(b2)

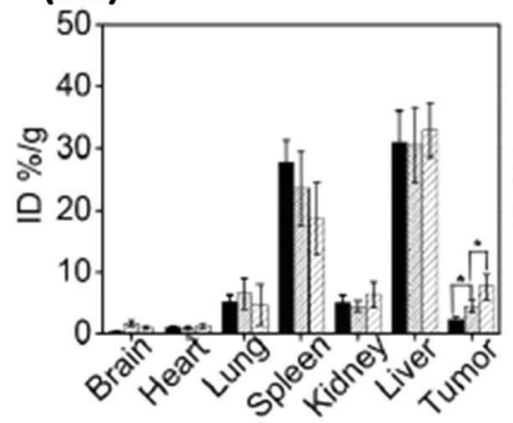

Time (h) (c1)

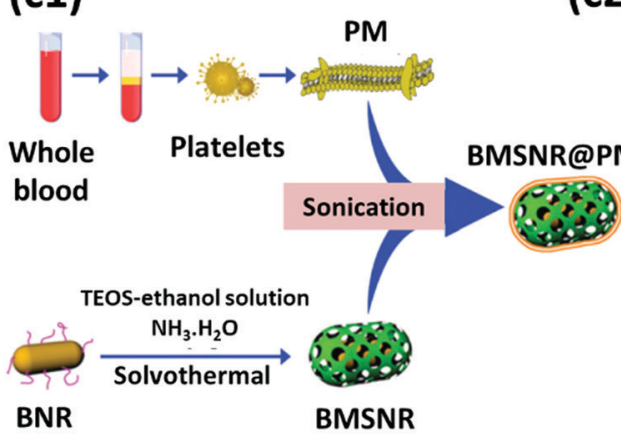

(c2) BMSNR

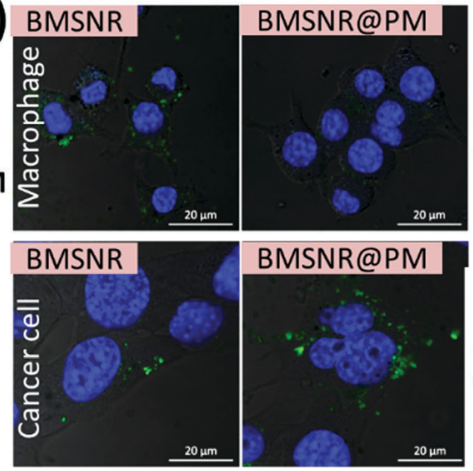

Hydrophobic Bi2S3 nanodots
Hydrophilic Bi2S3 nanodots

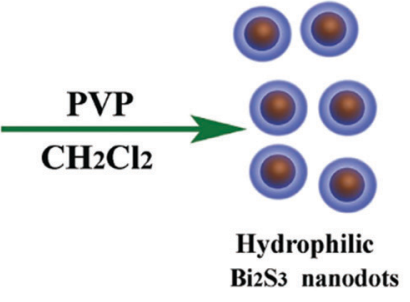

$=$ PVP coated Bi2S3 nanodots

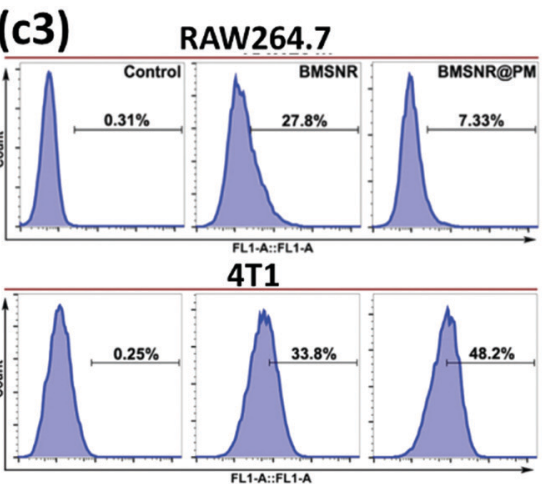

Fig. 10 (a1) A schematic representation of the synthesis and surface modification of oleic acid (OA) coated $\mathrm{Bi}_{2} \mathrm{~S}_{3}$ nanodots with PVP. (a2) Cytotoxicity of PVP-coated $\mathrm{Bi}_{2} \mathrm{~S}_{3}$ nanodots on HeLa cells. (a3) Histological changes in the spleen and kidney of a rat one month after the intravenous injection of a single dose of PVP-coated $\mathrm{Bi}_{2} \mathrm{~S}_{3}$ nanodots. No significant changes in the normal morphologies of the tissues were observed, indicating the high safety of the NPs. Adapted and reprinted with permission from ref. 222, Copyright 2011. (b1) The blood circulation of BiNPs (black curve), RBC-BiNPs (red curve) and F-RBC BiNPs (blue curve) over a span of $24 \mathrm{~h}$. (b2) The biodistribution of BiNPs (black), RBC-BiNPs (dense) and F-RBC BiNPs (sparse) in mice $24 \mathrm{~h}$ post-injection. Adapted and reprinted with permission from ref. 229, Copyright 2018. (c1) A schematic representation of BMSNR@PM synthesis. Bismuth sulfide nanorods (BNRs) were prepared via a solvothermal method. BNRs were decorated with mesoporous silica due to its well-defined structure and desirable biosafety. PM was separated from whole blood and applied to camouflage the $\mathrm{Bi}_{2} \mathrm{~S}_{3}$-mesoporous silica nanorods (BMSNRs) via sonication and to obtain BMSNR@PM. (c2) The endocytosis of BMSNR@PM by macrophages (RAW264.7) and cancer cells (4T1) after $4 \mathrm{~h}$ of exposure to nanomaterials. The increase in the cellular uptake by cancer cells after coating with PM is obvious. Blue and green signals indicate DAPI-stained nuclei and FITC-labeled nanomaterials, respectively. (c3) Flow cytometry of RAW264.7 macrophage cells and 4T1 mammary gland breast cancer cells after treatment with the nanomaterials for $4 \mathrm{~h}$. Increased cellular interactions after coating with PM are observed for cancer cells. Adapted and reprinted with permission from ref. 232, Copyright 2019

according to the criterion of the American Society for Testing and Materials (ASTM) standards, ${ }^{238}$ is the highest acceptable percentage of damaging potential of a foreign agent introduced into the body.

Various in vivo models have also been developed to test the toxicity of BiNPs. For example, Zheng et al. ${ }^{239}$ used a facile solvothermal method to prepare $\mathrm{Bi}_{2} \mathrm{~S}_{3}$ NRs by adding Bi neodecanoate (the precursor) to a mixture of oleic acid (OA) and ethanol under stirring (Fig. 11a1). NRs were then obtained by adding an oleylamine solution containing thioacetamide to the above mixture under vigorous stirring. The sealed samples were then heated at $150{ }^{\circ} \mathrm{C}$ for $10 \mathrm{~h}$ in an autoclave and finally cooled at room temperature to separate Bi2S3 NRs by centrifugation. The OA modified BRs were then further functionalized using amphiphilic $\mathrm{D}-\alpha$-tocopherol PEG 1000 succinate (TPGS) to enhance their aqueous compatibility. Finally, tetraethylorthosilicate (TEOS) 
(a1)
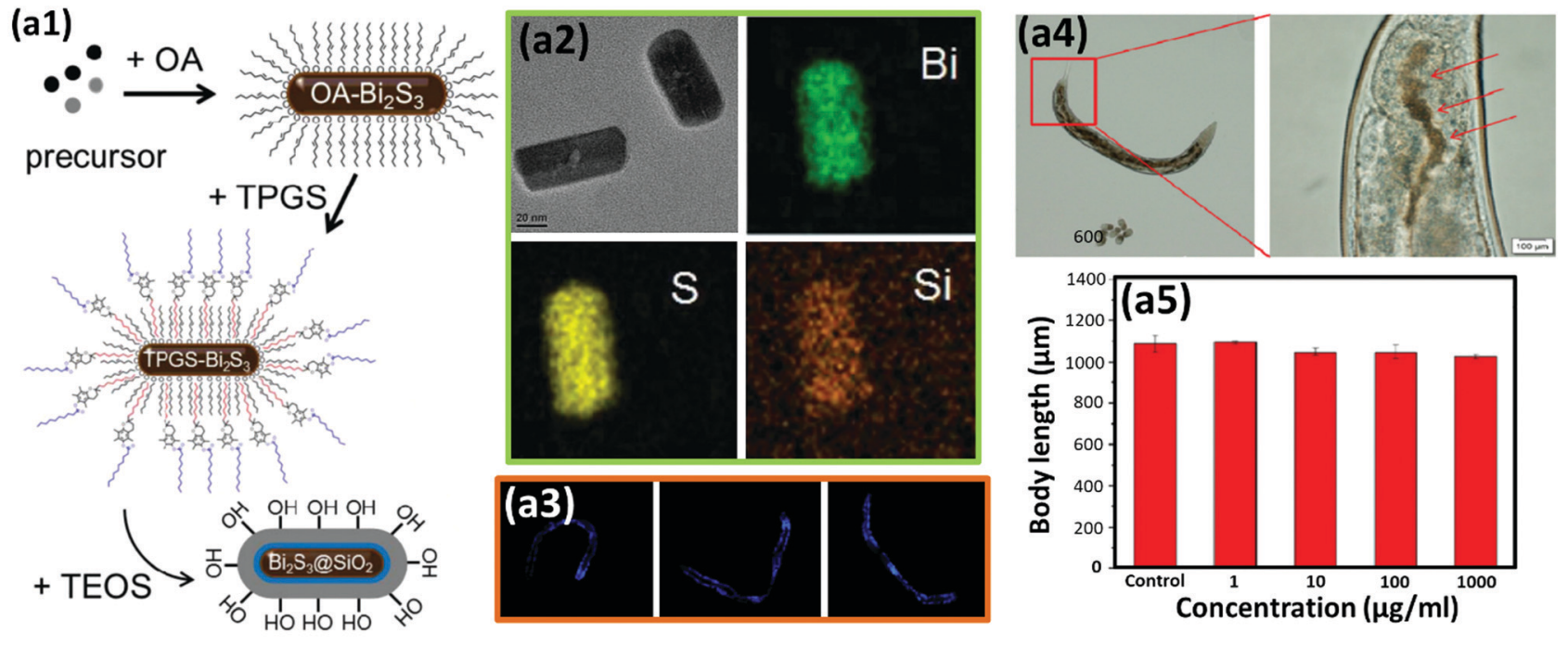

(b7)
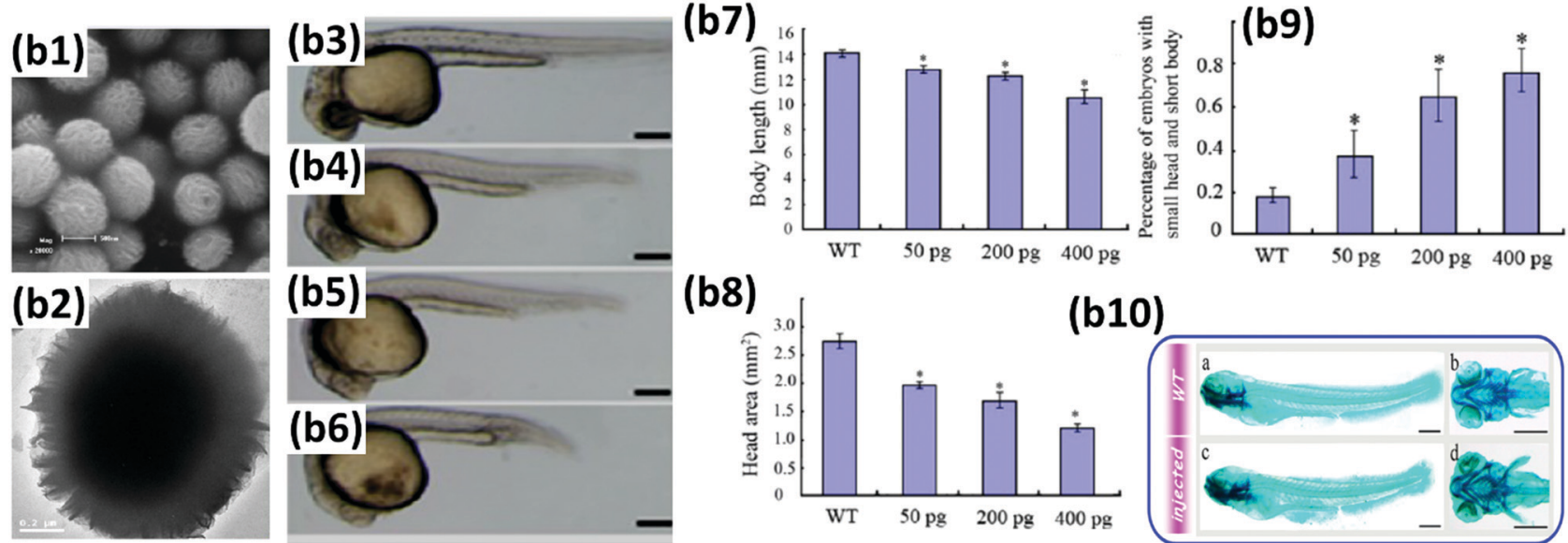

(b8)

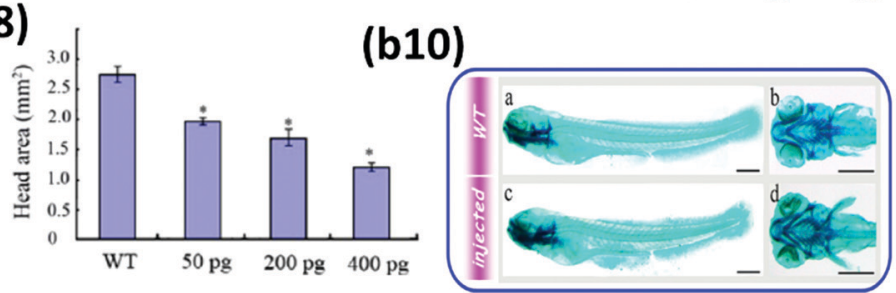

Fig. 11 (a1) A schematic illustration of the preparation process of $\mathrm{Bi}_{2} \mathrm{~S}_{3} \mathrm{CSiO}_{2}$ NRs for bimodal CT/PAT imaging of the Gl tract. (a2) A TEM image of $\mathrm{Bi}_{2} \mathrm{~S}_{3} @ \mathrm{CSiO}_{2} \mathrm{NRs}$ and the elemental mapping of $\mathrm{Bi}, \mathrm{S}$ and $\mathrm{Si}$ in $\mathrm{Bi}_{2} \mathrm{~S}_{3} @ \mathrm{aSiO}_{2} \mathrm{NRs}$. (a3) The effects of $\mathrm{Bi}_{2} \mathrm{~S}_{3} @ \mathrm{aSiO}_{2} \mathrm{NR}$ treatments at concentrations of 0 , 10, and $1000 \mu \mathrm{g} \mathrm{mL}^{-1}$ (from left to right) on the accumulation of fluorescent lipofuscin in age-synchronized worms. (a4) A bright field image of the $\mathrm{Bi}_{2} \mathrm{~S}_{3} @ \mathrm{ASiO}_{2}$ NR distribution in the Gl tract of $C$. elegans. (a5) The body lengths of $C$. elegans after treatment with $\mathrm{Bi}_{2} \mathrm{~S}_{3}\left(\mathrm{CSiO}_{2} \mathrm{NRs}_{\mathrm{N}}\right.$ with different concentrations. Adapted and reprinted with permission from ref. 239, Copyright 2015. (b1 and b2) SEM and TEM images of BACP-2. (b3-b6) Morphological characteristics of embryos at $28 \mathrm{hpf}$ (b3) without injection and (b4) with the injection of 50 pg, (b5) 200 pg, and (b6) 400 pg of BACP-2. (b7) Body lengths of uninjected embryos and embryos injected with BACP-2 at different concentrations at $28 \mathrm{hpf}$. (b8) The head areas of uninjected embryos and embryos injected with BACP-2 at different concentrations at $28 \mathrm{hpf}$. (b9) Percentages of embryos with a small head and short body at 28 hpf in uninjected embryos and embryos injected with different concentrations of BACP-2. (b10) Alcian blue staining of cartilage development at 5 dpf after the injection of BACP-2 at a concentration of $400 \mathrm{pg}$. (a and c) A lateral view with the anterior to the left. (b and d) A ventral view of the pharyngeal cartilage. Scale bars $=500 \mu \mathrm{m}$. Adapted and reprinted with permission from ref. 240, Copyright 2013.

was added dropwise to a solution of the TPGS-modified Bi2S3 NRs and the final product was obtained after 10 min of stirring, confirmed by elemental imaging of the particles (Fig. 11a2). After synthesis, the Caenorhabditis elegans (C. elegans) worm was used as a simple model organism to explore the safety and biological response of orally administered $\mathrm{Bi}_{2} \mathrm{~S}_{3} @ \mathrm{SiO}_{2} \mathrm{NRs}$. The accumulation of lipofuscin, an endogenous autofluorescent biomarker of aging and oxidative degeneration of cellular components, was investigated in C. elegans. Fig. 11a3 shows that after 3 days of the exposure of C. elegans to NRs with different concentrations, the autofluorescence intensity of the intestine was not notably changed between the control group and the groups treated with NRs, showing the negligible adverse effects of $\mathrm{Bi}_{2} \mathrm{~S}_{3} @ \mathrm{SiO}_{2} \mathrm{NRs}$ on the autofluorescence of lipofuscin and its levels within the worm body. Bright field imaging of the $\mathrm{Bi}_{2} \mathrm{~S}_{3} @ \mathrm{SiO}_{2}$ administered C. elegans was also performed to ensure the uptake of NPs into the gut of the worm (Fig. 11a4). In addition, no proliferation inhibition of the C. elegans and negligible changes in the body length after 18 days of exposure to various concentrations of the $\mathrm{Bi}_{2} \mathrm{~S}_{3} @ \mathrm{SiO}_{2}$ NRs up to $1000 \mu \mathrm{g} \mathrm{mL}^{-1}$ were observed (Fig. 11a5). As a result of the CT and photoacoustic tomography (PAT) imaging potential of the $\mathrm{Bi}_{2} \mathrm{~S}_{3} @$ $\mathrm{SiO}_{2} \mathrm{NRs}$, this study suggested that BiNPs provide an alternative way of fabricating multi-modal contrast agents that integrate different imaging modalities for direct and non-invasive visualization of the GI tract with minimal toxicity. 
In another study, zebrafish embryos were used for the toxicity assessment of Bi-asparagine coordination polymer spheres (BACP-2). ${ }^{240}$ Zebrafish is a promising animal model for drug formulation development because of the exclusive advantage of their genome similarity to the human genome, easy imaging of transparent embryos, well-characterized developmental stages, high fecundity, and low-cost husbandry and housing. ${ }^{241-243}$ Spherical BACP-2 with an average diameter of $c a .800 \mathrm{~nm}$ (Fig. 11b1 and b2) was easily synthesized by preparing a transparent solution of $\mathrm{Bi}$ nitrate $\left(\mathrm{Bi}\left(\mathrm{NO}_{3}\right)_{3} \cdot 5 \mathrm{H}_{2} \mathrm{O}\right)$ and asparagine, followed by $24 \mathrm{~h}$ of heating at $80{ }^{\circ} \mathrm{C}$. Zebrafish embryos at cell stages 1-4 cell were treated with 50, 200, and $400 \mathrm{pg}$ of BACP-2 through microinjection and their development was evaluated by observing the morphology of the embryos $28 \mathrm{~h}$ postfertilization (hpf) to 100 hpf. Fig. 11b3-b6 show smaller heads and shorter body lengths at $28 \mathrm{hpf}$ in the groups that received BACP-2 particles. Quantitative analysis demonstrated concentration-dependent toxicity as the percentages of embryos with shorter body lengths and smaller heads constantly increased from $20 \%$ in the control group to $38 \%, 65 \%$, and $77 \%$ in the groups that were injected with 50, 200, and $400 \mathrm{pg}$ of BACP-2, respectively (Fig. 11b7-b9). However, the evaluation of cartilage development by alcian blue staining 5 days postfertilization (dpf) demonstrated no harmful effect of the BACP-2 compared to the uninjected embryos of the control group (Fig. 11b10). While the toxicity of BACP-2 on zebrafish embryos was obvious, another study showed that the number of viable chicken embryos remained unchanged after treatment with $\mathrm{Bi}_{2} \mathrm{O}_{3}$ /human serum albumin (HSA) core-shell NPs, indicating normal functioning of embryos after exposure to these NPs. ${ }^{244}$ These findings provide new insights into the possible controversies and the necessity of rigorous toxicity assessment for all types of BiNPs before their application in biomedicine.

Murine models have also been used to investigate the in vivo safety of BiNPs. For example, PVP coated $\mathrm{Bi}_{2} \mathrm{Se}_{3}$ nanoplates were tested at a high dose of $20 \mathrm{mg} \mathrm{kg}^{-1}$ in C57 male mice through intraperitoneal injection, showing 93\% metabolism and clearance within 90 days. $^{245}$ The liver, spleen, and kidneys were the main target organs for $\mathrm{Bi}$ accumulation, reaching concentrations of $163.2,101.9$, and $46.8 \mu \mathrm{g} \mathrm{g}^{-1}$ in the aforementioned organs after 1 day, respectively. Reticuloendothelial systems (RES) play a main role in the dominant accumulation of nanoplates within the liver and spleen compared to the other organs. ${ }^{245}$ In general, no meaningful statistical differences were observed over a 90 day period on the growth, body weight, and thymus index of the animals, as well as the hematology markers, biochemistry markers, and histology of various organs between the nanoplate-treated mice and the control group. Even small changes were negligible since the damages were recovered to the normal state over the monitoring period of 90 days. For example, a meaningful change in the blood level of alanine aminotransferase (ALT), as an indicator of hepatic function, was observed on day 7 and recovered to the normal level over the next screening time-points of 30 and 90 days. Comparing the above system with other types of nanomaterials, such as gold NPs (AuNPs) stabilized by PEG and bovine serum albumin (BSA) ${ }^{246,247}$ or $\mathrm{Fe}_{3} \mathrm{O}_{4}$ nanocrystals coated with PEG or $\mathrm{OA},{ }^{248}$ shows that coated BiNPs cause much less acute damage and changes in the biochemistry parameters of the blood. Another interesting study by Zhang et al. ${ }^{249}$ demonstrated not only the high biocompatibility of PVP coated $\mathrm{Bi}_{2} \mathrm{Se}_{3}$ NPs at a high dose of $50 \mathrm{mg} \mathrm{kg}^{-1}$ in mice, but also their capability to protect animals against ionizing radiation via their free radical scavenging effect and electrocatalytic properties. In blood circulation, $\mathrm{Bi}_{2} \mathrm{Se}_{3}$ NPs are prone to oxidation, and therefore, they can provide a strong reducing effect for protection against ROS and lots of free radicals generated by exposure to ionizing radiation during the radiotherapy of cancer. It was found that while the survival fraction of mice exposed to $662 \mathrm{keV}$ gamma photons was $0 \%$, treatment with $\mathrm{PVP}-\mathrm{Bi}_{2} \mathrm{Se}_{3} \mathrm{NPs}$ protected the animals from gamma radiation and increased the survival rate to $70 \%$. Additionally, NPs were gradually excreted for up to 90 days without any significant side effects in panels of clinical biochemistry, blood chemistry, and pathology at a dose of $50 \mathrm{mg} \mathrm{kg}{ }^{-1}$. These NPs also improved the recovery of the radiation mediated fall in the level of RBCs, white blood cells (WBCs), and platelets. Promisingly, $\mathrm{Bi}_{2} \mathrm{Se}_{3}$ NPs also induced an increase in superoxide dismutase (SOD; an enzyme that alternately catalyzes the dismutation of superoxide radicals into either ordinary molecular oxygen or hydrogen peroxide) and reduction of 3,4-methylenedioxyamphetamine (MDA; a harmful byproduct from mice) in animals exposed to ionizing radiation. Therefore, it was conceived that $\mathrm{Bi}_{2} \mathrm{Se}_{3}$ NPs have great potential to be used as adjuvant therapy to suppress radiation-induced injuries. In fact, the Se released from the particles was hypothesized to be able to reduce the side effects of RT through the improved activity of glutathione peroxidase (GSH-Px) and SOD, improving the radioresistance of healthy tissue. ${ }^{250}$ However, to corroborate and extend the medical applications of BiNPs towards this area, further in vivo explorations are needed.

The biosafety of nanomaterials, including BiNPs, is directly related to their elimination from the body after administration. ${ }^{251}$ Clearance through the renal (urine) and hepatic (bile to feces) pathways are two main routes for NP excretion. ${ }^{252}$ Renal clearance needs glomerular filtration of nanomaterials in the kidneys, a phenomenon that is highly dependent on the shape, charge, and size of NPs. The typical filtration-size threshold (FST) of the capillary walls of the glomerulus is nearly $6 \mathrm{~nm}$. This means that the filtration of nanomaterials with hydrodynamic diameters larger than FST is not possible by the kidneys, unless they degrade into smaller residues. If not, the Kupffer cells of RES capture large NPs and digest them for clearance through the hepatic pathway and later excrete them in feces. Most Bi-based NPs possess a diameter larger than the FST threshold, ${ }^{38,50,121,253-256}$ therefore, they cannot pass through the glomerular capillary walls and be eliminated in urine. Even BiNPs with a size smaller than $6 \mathrm{~nm}$ can become larger in biological environments due to the possible adsorption of plasma proteins on their surface. For example, the size of $\mathrm{Bi}_{2} \mathrm{~S}_{3}$ NPs has been shown to increase from 6.1 to $39.52 \mathrm{~nm}$ after BSA adsorption on their surface. ${ }^{257}$ Accordingly, similar to many other types of nanovehicles, hepatic clearance seems to be the dominant route of elimination for 
BiNPs due to their large hydrodynamic diameter. ${ }^{53,258}$ In general, BiNPs with any hydrodynamic size larger than the FST, various shapes, different surface coating and chemical composition have shown dominant accumulation in the liver shortly after intravenous injection..$^{229,232,245}$ However, the synthesis of very small particles, surface coated with proteinadsorption-resistant materials or using rapidly degradable BiNPs might help to alter the clearance pathway towards the urinary system. $^{237,245,250,259}$ For example, Bi was easily detectable in kidneys $2 \mathrm{~h}$ after the injection of dextran coated Bi-iron oxide nanohybrid. ${ }^{260}$ This shows that the Bi was efficiently cleared through the urinary route as a result of particle degradation in the acidic environment. The level of $\mathrm{Bi}$ in the urine was found to be much higher than that of Fe due to the confiscation of released Fe ions by ferritin for the synthesis of hemoglobin, whereas such a system does not exist for the consumption of $\mathrm{Bi}$ ions in the body. In addition, the incubation of the nanohybrid in a $10 \%$ solution of fetal bovine serum reduced their size from 98 to $48 \mathrm{~nm}$ after $24 \mathrm{~h}$ as a result of time-dependent degradation in biological fluids, confirmed by the detection of free $\mathrm{Bi}$ ions in the bio-mimicking media.

Similar to the above example, the accumulation of $\mathrm{Cu}_{3} \mathrm{BiS}_{3}$ NPs in the urinary system and their renal clearance were dominant. ${ }^{259}$ This observation can be attributed to the small size of the particles as well as the rapid chemical transformation and degradation of the $\mathrm{Cu}_{3} \mathrm{BiS}_{3}$ into $\mathrm{Cu}-\mathrm{COO}^{-}$species in acidic lysosomal environment. Clearance through the kidneys was also observed for $(\mathrm{BiO})_{2} \mathrm{CO}_{3}$ nanotubes due to their rapid disassembly in acidic environments, such as tumor tissue, to nanoclusters with a size smaller than the FST, resulting in their facile renal filtration within a short time post administration. ${ }^{261}$ This study showed that the Bi content excreted via the renal route reached $c a$. 35\% within $12 \mathrm{~h}$ post injection, while fecal clearance was lower than $2 \%$ during the same time period. In contrast to the above examples that show renal clearance of some BiNPs due to particle degradation, $\mathrm{Bi}-\mathrm{Bi}_{2} \mathrm{~S}_{3}$ heterostructure NPs were removed from the body through urine because of their own ultrasmall size. ${ }^{237}$ Therefore, further assessments are necessary to understand the optimal size and shape of BiNPs, which might guide their clearance towards the renal system. Moreover, comprehensive studies on the role of the route of administration and an understanding of the relationship between the surface coating of BiNPs and their clearance are still required.

\section{Antimicrobial activity of BiNPs and their composites}

As the field of metal-based nanomedicine emerges, there is an overwhelming number of studies on research surrounding the topic of antimicrobial NPs due to the continuous occurrence of bacterial resistance, which is a life-threatening challenge worldwide. ${ }^{4,262-264}$ Nowadays, it is believed that some categories of metallic NPs are good antimicrobial agents, because of their high activity at low doses and diverse bactericidal effects, providing the desirable potential to reduce or eliminate the evolution of resistant bacterial strains. ${ }^{265}$ Mechanisms involved in the antimicrobial effect of metal-based NPs include the production of ROS, cation release from the NPs, ATP depletion, membrane impairment and alteration of its respiratory function, protein dysfunction and interference with nutrient assimilation. These mechanisms even translate into alteration of signal transduction and the inducing of destructive changes to the microbial transcriptomic and proteomic profiles through dephosphorylation of the peptide substrates on tyrosine residues, resulting in the inhibition of signal transduction and suppression of bacterial growth. ${ }^{262,266-269}$

The development and application of $\mathrm{Bi}$ in diverse forms such as Bi salts, NPs, and nanocomposites as antimicrobials have been increasingly investigated due to the biocompatibility of $\mathrm{Bi}^{270}$ creating new hope to fight infectious diseases and bacterial resistance. ${ }^{163,271-279}$ For example, a combination of $\mathrm{Bi}$ organic salts and some antibiotics are currently used to manage Helicobacter pylori (H. pylori) infections. ${ }^{280,281}$ Likewise, Bi carboxylates and Bi thiols show a capacity to treat $H$. pylori and to inhibit biofilm formation. ${ }^{282,283}$ Hernandez-Delgadillo et al. ${ }^{273,274}$ reported the great antifungal and antibacterial properties of BiNPs at low concentrations of $2 \mathrm{mM}$ and less than $1 \mathrm{mM}$, respectively. They showed the inhibitory effect of zerovalent BiNPs against the growth of Streptococcus mutans (S. mutans) with a minimal inhibitory concentration (MIC) of $0.5 \mathrm{mM}$ and their ability to inhibit biofilm formation similar to the antimicrobial effect exhibited by chlorhexidine. The mechanism of action of these zerovalent BiNPs was not described, but hypothesized to be associated with the high surface-to-volume ratio of the particles, their quantum confinement, their stability in aqueous media, and irregularities present in the crystal lattice of the nanostructured BiNPs that provide more active sites to interact with bacteria. ${ }^{273,274}$ Badireddy et al., ${ }^{163,275}$ reported colloidal Bi dimercaptopropanol (BisBAL) with an inhibitory effect on the biofilm formation of Pseudomonas aeruginosa (P. aeruginosa) and the inhibiting of the growth of $S$. mutans and $S$. gordonii by over $70 \%$ at $0.1 \mu \mathrm{M}(\mathrm{MIC}=5 \mu \mathrm{M})$. The mechanism of antibacterial activity of the BisBAL NPs was postulated to be related to the dissolution of the BiNPs and release of the Bi ions, which could penetrate to the cell wall and then react with the sulfhydryl groups of key respiratory chain enzymes to inactivate and lyse bacteria. ${ }^{275}$ Similarly, Hernandez-Delgadillo et al. ${ }^{284}$ recently incorporated BisBAL NPs into a mineral trioxide aggregate (MTA) to reduce the risk of infection, when MTA was used in endodontic therapy. The Kirby and Bauer disk diffusion method showed that the growth of Enterococcus faecalis (E. faecalis), E. coli, and C. albicans could effectively be inhibited by BisBAL NPs, demonstrating inhibition zones of 19, 24, and $23 \mathrm{~mm}$, respectively. BisBAL NPs also showed antibiofilm activity against $E$. faecalis at a concentration of $10 \mathrm{mg} \mathrm{mL}$ and exposure time of $24 \mathrm{~h}$, suggesting that they are a good candidate to decrease the incidence of dental re-infections after the use of MTA in endodontic therapy. To highlight the role of nanosized BiNPs, Cabral-Romero et al. ${ }^{274}$ showed the better antimycotic effects of $\mathrm{Bi}_{2} \mathrm{O}_{3}$ NPs against $C$. albicans compared 
to other forms of $\mathrm{Bi}$, including bulk polycrystalline $\mathrm{Bi}_{2} \mathrm{O}_{3}$ and its precursor $\mathrm{Bi}$ nitrate, $\mathrm{Bi}\left(\mathrm{NO}_{3}\right)_{3}$, suggesting that the high surface area of the nanostructured $\mathrm{Bi}_{2} \mathrm{O}_{3}$ was responsible for its antimycotic activity, with a MIC of $1.5 \mathrm{mM}$. The hypothesis for the antifungal mechanism of the $\mathrm{Bi}_{2} \mathrm{O}_{3}$ NPs relies on the increased amount of water molecules and $\mathrm{OH}^{-}$ions bound to the surface of the particles, creating a highly negative surface charge and basic aggregation points, to which C. albicans is highly sensitive. Nevertheless, other mechanisms might be involved in the antimicrobial potential of BiNPs. For example, Nazari et al. ${ }^{276}$ prepared biogenic BiNPs through the inoculation of Bi subnitrate with a pure culture of Serratia marcescens and then demonstrated the multifaceted antimicrobial potential of the particles against different strains of Gramnegative $H$. pylori. An inhibitory dose of biogenic BiNPs (100 $\mu \mathrm{g} \mathrm{mL} \mathrm{mL}^{-1}$ ) caused the release of metabolites, such as acetate, formic acid, glutamate, valine, glycine, and uracil from $H$. pylori bacteria into their supernatant, suggesting that the antimicrobial activity of BiNPs results from their interference of the Krebs cycle, nucleotides, and acid metabolism. This multifaceted antimicrobial potential is the main reason for using $\mathrm{Bi}$ subsalicylate NPs (BSS-NPs) in dental materials or antiseptic solutions against oral anaerobic bacteria (e.g., Actinomyces israelii, Aggregatibacter actinomycetemcomitans serotype b, Capnocytophaga gingivalis, Eikenella corrodens, Fusobacterium nucleatum subsp. nucleatum, Parvimonas micra, Porphyromonas gingivalis, Prevotella intermedia, Streptococcus mutans, and Streptococcus sanguinis ${ }^{278}$ or preparing Bi-substituted hydroxyapatite nanopowders for potential applications in bone infection and regeneration. ${ }^{285}$ Moreover, BiNPs and Bi nanocomposites may also be used for combinatorial therapy of microbial infections by taking advantage of the radiosensitizing properties of $\mathrm{Bi},{ }^{277}$ which can be combined with the antimicrobial effect of antibiotics. $^{286,287}$ Such synergistic effects may be an effective way to kill multidrug-resistance bacteria through inducing irreparable DNA damage due to the free radicals generated by X-ray irradiation. ${ }^{198}$ Luo et al. ${ }^{277}$ have developed BiNPs surfacemodified with anti- $P$. aeruginosa antibodies to impart them with selective X-ray mediated toxicity towards $P$. aeruginosa due to the specific binding of the NPs to the bacteria, representing a promising approach to accelerate the healing process in bacterial infected wounds. In addition to X-ray mediated antibacterial effects, Sharma et $a .^{288}$ described visible the lightinduced bactericidal effects of octahedral monoclinic-Bi vanadate $\left(\mathrm{m}-\mathrm{BiVO}_{4}\right) \mathrm{NPs}$ towards $E$. coli. Similarly, the light-induced ROS generation and photodynamic antibacterial effects of BiNPs were also reported for nitrogen-rich carbon-coated $\mathrm{Bi}$ / cobalt (Bi@Co@CN) NPs. ${ }^{289}$ Particles were fabricated via a thermal condensation method using $\mathrm{Bi}\left(\mathrm{NO}_{3}\right)_{3}$ and $\mathrm{Co}\left(\mathrm{NO}_{3}\right)_{2}$ metal salts (Fig. 12a). Graphitic carbon nitride $\left(\mathrm{g}-\mathrm{C}_{3} \mathrm{~N}_{4}\right)$ is an antibacterial agent and the energy created by electron-hole pairs enables it to generate ROS. However, rapid electron-hole recombination limits its application as a potent antibacterial agent. To overcome this limitation, g- $\mathrm{C}_{3} \mathrm{~N}_{4}$ was decorated with BiNPs, which has a narrow bandgap enabling it to harvest visible light to generate thermal energy for a better antimicrobial effect. Furthermore, Bi on the surface of $\mathrm{g}-\mathrm{C}_{3} \mathrm{~N}_{4}$ can facilitate charge transfer and accelerate electron-hole separation, leading to enhanced photocatalytic activity. Co was also used in this system because of its high photothermal conversion effect to reduce the chance of infection recurrence after using Bi@Co@CN as an antibacterial agent. In vitro findings demonstrated the excellent antibacterial activity of Bi@Co@CN against both Gram-positive ( $S$. aureus) and Gramnegative (E. coli ATCC25922 and Salmonella LT2) bacteria, as well as methicillin-resistant Staphylococcus aureus (MRSA). Within $20 \mathrm{~min}$ of treating a $S$. aureus suspension with the Bi@Co@CN composite $(2 \mathrm{mg}$ of the composite in $10 \mathrm{~mL}$ of bacterial suspension containing $c a \cdot 10^{7}$ colony forming units per $\mathrm{mL}$ ) under visible-light irradiation, more than $99.9 \%$ of the bacteria were killed due to the abundant generation of ROS and thermal energy, imparting damage to the bacterial membranes (Fig. 12b). Excellent charge separation efficiency between the $\mathrm{g}-\mathrm{C}_{3} \mathrm{~N}_{4}$ nanostructures and metal NPs provided a desirable antibacterial environment. In contrast, negligible antimicrobial activity was observed in the dark and light controls. The disinfection efficiencies of $\mathrm{Bi} @ \mathrm{CN}$ and $\mathrm{Co@CN}$ were also significantly less than that of the Bi@Co@CN composite. Fig. 12c displays the colonies of $S$. aureus on an agar plate after exposure to Bi@Co@CN at various irradiation times, confirming the gradual killing of the bacteria with increasing illumination time. The role of ROS generation in the treatment of MRSA infected wounds was also assessed in vivo, demonstrating very effective wound healing without detectable damage to the major organs (Fig. 12d and e). The suggested mechanism of action is the inhibition of the expression and transcription of virulence factors and the inhibition of the transcription of methicillin resistant gene (mecA) of MRSA mediated by ROS generation.

The same concept of inducing ROS generation was also reported by $\mathrm{Hsu}$ et al., ${ }^{290}$ by preparing biocompatible BiOI nanosheets with high photocatalytic activity and energy storage efficiency, and then, conducting in situ-doping of AuNPs and gold-iodide (Au-I) complexes into BiOI nanosheets to render the nanocomposites with high oxidase-like activity. ${ }^{291}$ Certain volumes of $\mathrm{Bi}\left(\mathrm{NO}_{3}\right)_{2}(10 \mathrm{mM}), \mathrm{KI}(10 \mathrm{mM})$, and $\mathrm{HAuCl}_{4}$ (1, 10 or $100 \mathrm{mM}$ ) were added to a mixed solution containing ultra-pure water and tris-borate buffer and this was allowed to react via shaking in the dark for $2 \mathrm{~h}$ to form $\mathrm{Au} / \mathrm{BiOI}$ nanocomposites. Doping of AuNPs disrupted the layered crystalline structures of BiOI, leading to the formation of a high degree of oxygen vacancies and 4-fold higher oxidase-like activity compared to the $\mathrm{Bi}$ nanosheets alone. In addition, the doping of AuNPs reduced the MIC of the BiOI NPs from more than $400 \mu \mathrm{g} \mathrm{mL}^{-1}$ to $c a$. $0.1-0.2 \mu \mathrm{g} \mathrm{mL}^{-1}$. The very low MIC range of the $\mathrm{Au} / \mathrm{BiOI}$ nanocomposite, which indicates its highly efficient antibacterial activity, was obtained for various bacteria, including $E$. coli, K. pneumoniae, S. enteritidis, S. aureus, B. subtilis, and MRSA. This was mainly due to the strong interactions of the nanocomposite with the bacterial membrane and higher generation of ROS through the strong oxidase-like activity. The ROS generation induced bacterial killing via the destruction of cell 


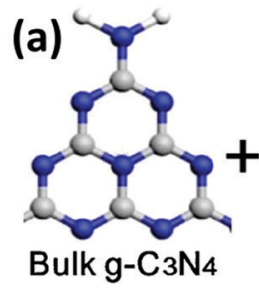

(b) 8

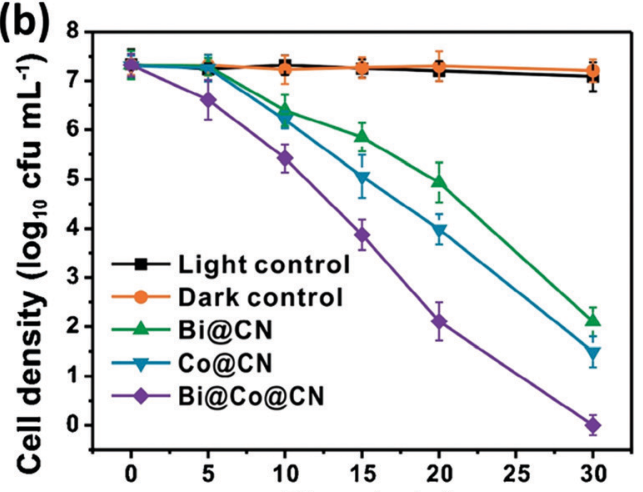

(d) Time (min)

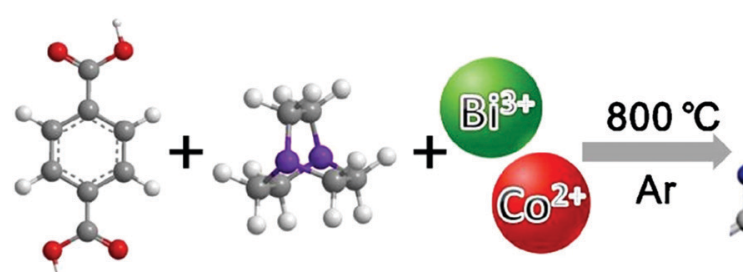

(c)

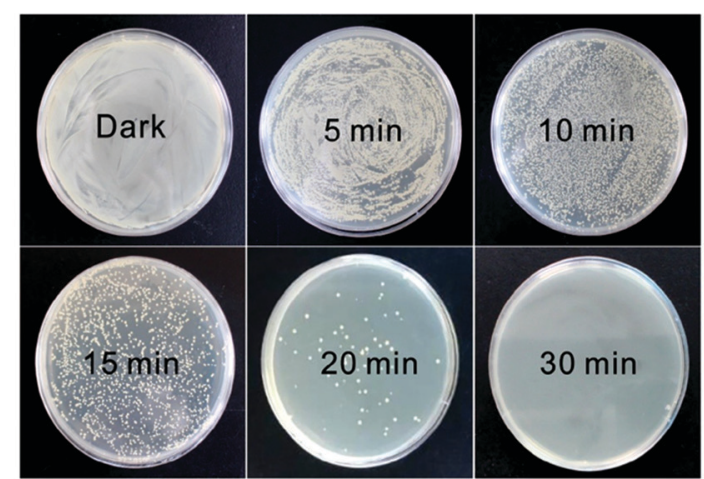

(e) PBS

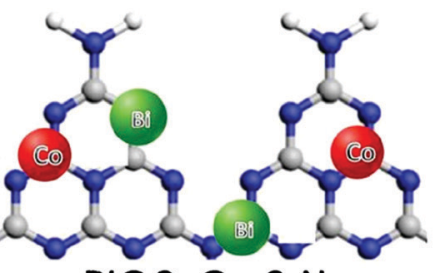

Bi@Co@g-C3N4

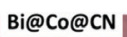

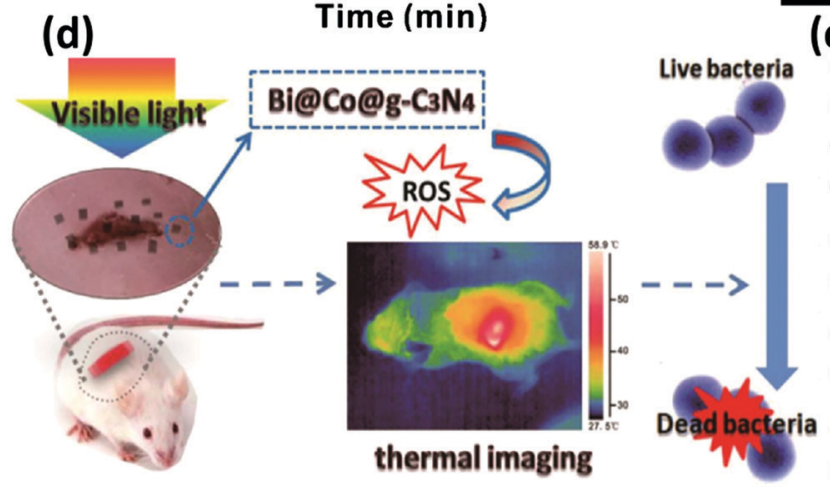

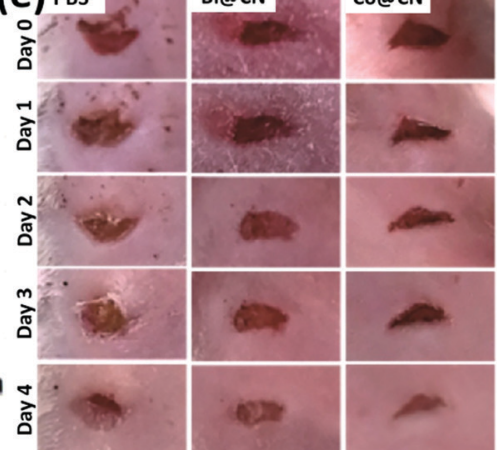

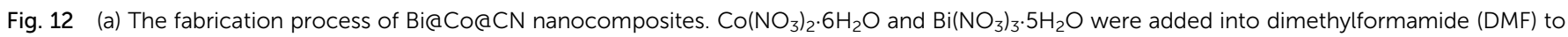

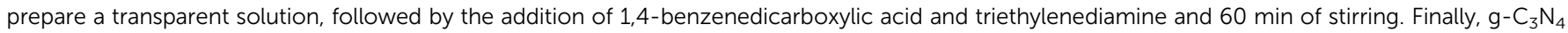

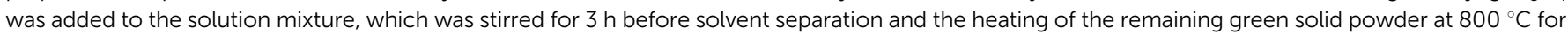

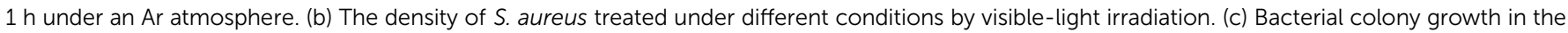

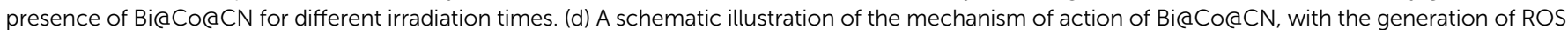

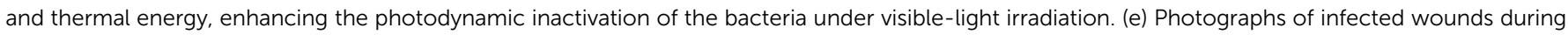

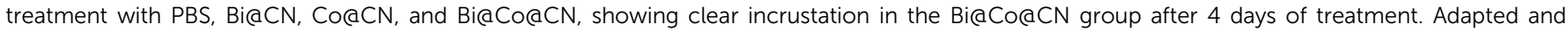
reprinted with permission from ref. 289, Copyright 2019.

membrane, inducing DNA and protein damage and disrupting the electron transport of the bacterial membrane and respiration processes. In this study, a $S$. aureus-infected bacterial keratitis model was developed in rabbits to prove the effectiveness of the nanocomposite on ocular infections. When compared with Ag NPs, Au/BiOI nanocomposites showed better maintenance of corneal thickness and lamellar structure of the stromal layer, less inflammation, and higher biocompatibility.

In addition to ROS generation mediated by the photodynamic antibacterials (PDA) discussed in the above examples, photothermal antibacterials (PTA) of BiNPs have been recently introduced to eradicate bacterial infections through the irradiation of NIR (700-1100 nm) light. ${ }^{292}$ Light-to-heat conversion by BiNPs can synergize the killing effect of ROS generation and eradicate bacterial infections (Fig. 13a1). Novel $\mathrm{Bi}_{2} \mathrm{Se}_{3}$ nanoplates (Fig. 13a2) were fabricated via a high-temperature reaction (colloidal synthesis) and their surface was coated with polyethyleneimine (PEI) through electrostatic interactions. ${ }^{293}$ These NPs, at a concentration of 80 parts per million (ppm), were found to eradicate nearly $99 \%$ of $S$. aureus and around $97 \%$ of $E$. coli bacteria upon 10 min of NIR light irradiation through combined PTT and photodynamic therapy (PDT) via the generation of heat and ROS, respectively (Fig. 13a3 and a4). Li et $a .^{294}$ followed the same concept for the combined dualmodal therapy of wounds infected by multidrug-resistant (MDR) bacteria, using multi-functional heterojunctions of sheet-like zinc atom-doped g- $\mathrm{C}_{3} \mathrm{~N}_{4}$ and $\mathrm{Bi}_{2} \mathrm{~S}_{3}$ nanorods $(\mathrm{CN}-\mathrm{Zn} / \mathrm{BiS})$, which were formed via the interspersion of clubbed BiS nanorods in $\mathrm{CN}$. Excellent photothermal properties of $\mathrm{CN}-\mathrm{Zn} / \mathrm{BiS}$ under $808 \mathrm{~nm}$ NIR irradiation were confirmed by observing stable temperature variation and nearly constant maximum temperature during the on-off effects of the NIR laser. $\mathrm{CN}$ and $\mathrm{CN}-\mathrm{Zn}$ 


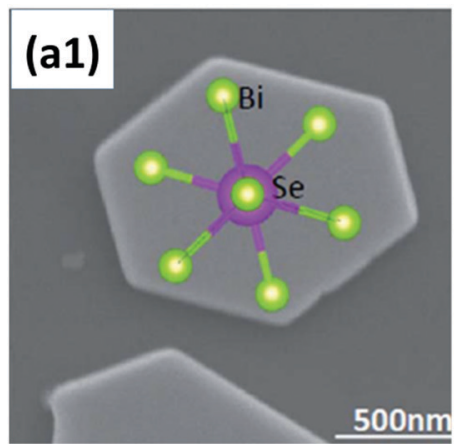

(b1)

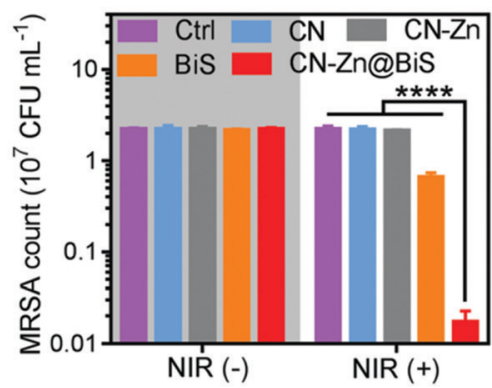

(b2)
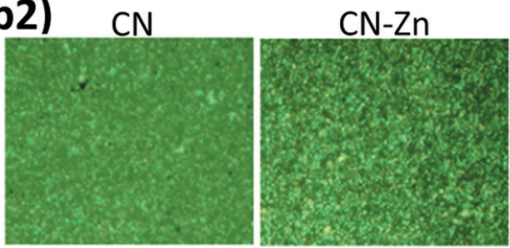

BiS

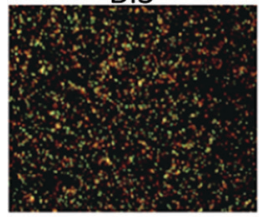

$\mathrm{CN}-\mathrm{Zn} / \mathrm{BiS}$
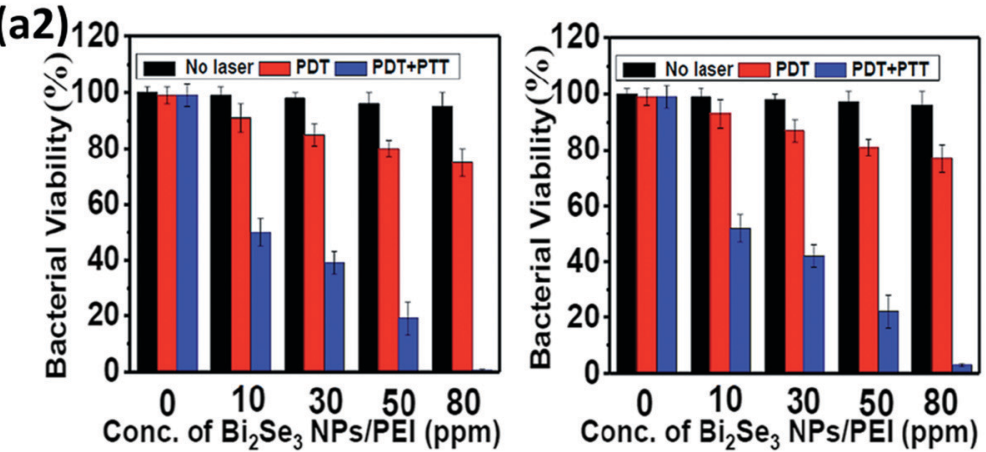

(b3)
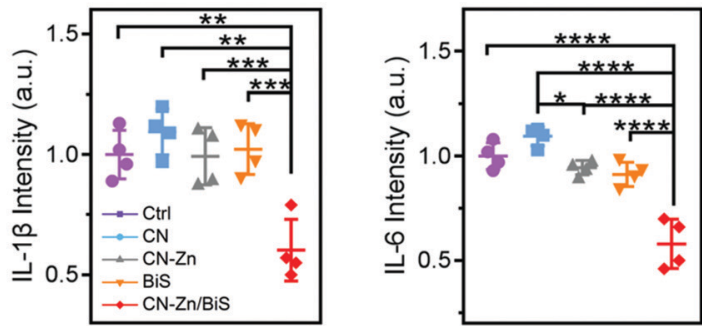

(b4)

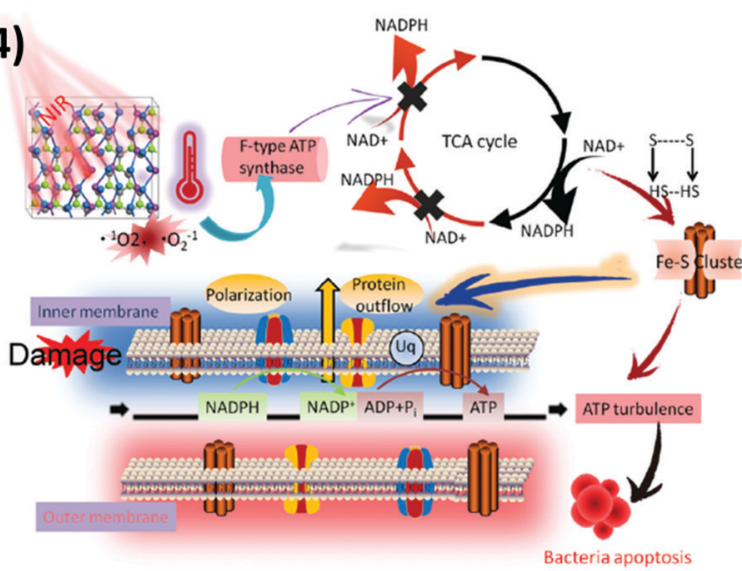

Fig. 13 (a1) A high-magnification SEM image of a $\mathrm{Bi}_{2} \mathrm{Se}_{3}$ NP. (a2) The antibacterial effects of PEI coated $\mathrm{Bi}_{2} \mathrm{Se}_{3} \mathrm{NPs}$ as a function of concentration. Bacterial viability is presented as a percentage under the different conditions of no laser, PDT alone and combined PDT and PTT for S. aureus (left graph) and E. coli (right graph). Adapted and reprinted with permission from ref. 293, Copyright 2018. (b1) MRSA strain counts calculated from spread-plate assays after exposure to $\mathrm{CN}, \mathrm{CN}-\mathrm{Zn}$, BiS, or $\mathrm{CN}-\mathrm{Zn} / \mathrm{BiS}\left(200 \mu \mathrm{g} \mathrm{mL}^{-1}\right)$ under $808 \mathrm{~nm}$ NIR light irradiation. (b2) Live/dead fluorescence staining of MRSA. Green fluorescence from live bacteria stained using SYTO9 dye and red fluorescence from dead bacteria stained using propidium iodide (PI) dye were detected. (b3) The quantification of inflammatory IL-1 13 and IL- 6 cytokines in wound tissues after 2 days of treatment with CN, CN-Zn, BiS, or CN-Zn/BiS. (b4) The antibacterial mechanism of PDA/PTA induced by $\mathrm{CN}-\mathrm{Zn} / \mathrm{BiS}$ under $808 \mathrm{~nm}$ light irradiation. Adapted and reprinted with permission from ref. 294, Copyright 2019.

did not show any significant killing effect towards MRSA at a concentration of $200 \mu \mathrm{g} \mathrm{mL} \mathrm{m}^{-1}$ under $808 \mathrm{~nm}$ NIR irradiation. In contrast, $70.3 \%$ and over $99.2 \%$ of MRSA were killed under the same conditions after exposure to BiS and $\mathrm{CN}-\mathrm{Zn} / \mathrm{BiS}$, respectively (Fig. 13b1 and b2). The nanocomposite also regulated inflammatory factors, such as IL-1 $\beta$ and IL-6 in vivo (Fig. 13b3) to accelerate the regeneration of infected wounds after killing the bacteria via NIR therapy. Fig. 13b4 summarizes the combined PDA/PTA antimicrobial mechanisms of the $\mathrm{CN}-\mathrm{Zn} / \mathrm{BiS}$ nanocomposite. The pernicious effects of ROS and hyperthermia decreased F-type ATP and increased ortho-nitrophenyl- $\beta$-galactoside (ONPG) hydrolysis, resulting in damage to electron transport in the respiratory chain, membrane polarization and protein leakage from the bacterial wall. It is worth pointing out that combined antibacterial and anti-inflammatory effects, similar to the above example have also been reported by Ouyang et al. ${ }^{295}$ who showed selective cell wall disruption and eradication of Gram-positive $S$. aureus bacteria by $\mathrm{Bi}_{2} \mathrm{Se}_{3}$ nanodiscs $\left(50 \mu \mathrm{g} \mathrm{mL}{ }^{-1}\right)$ due to the high affinity of the nanodiscs towards lipoteichoic acid on the surface of the bacteria. Furthermore, $\mathrm{Bi}_{2} \mathrm{Se}_{3}$ was found to be capable of scavenging the intracellular ROS of macrophages and reducing inflammation through a decrease in inflammatory cytokines, endowing them with high efficacy to treat infected wounds. Nevertheless, there is a controversy between these two studies due to the lack of information on the work performed by Li et al. While Ouyang and his team achieved anti-inflammatory 
responses through reduced ROS production by $\mathrm{Bi}_{2} \mathrm{Se}_{3}$, Li et al. used $\mathrm{CN}-\mathrm{Zn}$ /BiS for NIR-mediated ROS production for bacterial killing, while still demonstrating reduced secretion of inflammatory cytokines, such as interleukin (IL)-1 $\beta$ and IL-6. In this study, we were not able to realize whether the anti-inflammatory tests were performed after treatment with $\mathrm{CN}-\mathrm{Zn} / \mathrm{BiS}$ alone or in combination with NIR irradiation and subsequent ROS production, which presumably increases the secretion of inflammatory cytokines instead of their suppression. Therefore, further studies are needed to identify the main aspects of the effects of $\mathrm{Bi}$ on immune reactions.

In addition to its nanoform, Bi may also be incorporated in other structures such as nanocellulose matrices, ${ }^{296}$ nanoneedles, ${ }^{297}$ and implants ${ }^{298-300}$ for antimicrobial applications. As a result of the known antimicrobial properties of $\mathrm{Bi}$, and since organophosphorus compounds, in particular phosphinic acids, are known for their antibacterial properties, ${ }^{301}$ Werrett et al. ${ }^{296}$ investigated the synergistic effects of $\mathrm{Bi}^{(\mathrm{III})}$ and phosphinate ligands towards MDR bacteria, by incorporating Bi-phosphinato complexes into microfibrillated nanocellulose to prepare Bi-cellulose paper sheets. The Bi-paper composites showed potent antibacterial activity against different bacteria ( $S$. aureus, MRSA, vancomycinresistant enterococci, E. coli). The Gram-positive bacteria were found to be more susceptible than Gram-negative E. coli, probably due to the double membrane of the latter. Interestingly, for all bacterial strains except $E$. coli, the bi-phosphinato complex composites outperformed the silver standard and their meaningful potential for usage in surface coatings, packaging and/or medical devices were suggested. As another example, Indurkar et al. ${ }^{297}$ fabricated $\mathrm{Bi}_{2} \mathrm{O}_{3}$ nanoneedles with an average size of $158 \mathrm{~nm}$ and lengths of 1-3 $\mu \mathrm{m}$ via a green route using D. alata tuber extract for the first time. The antifungal activity of the nanoneedles ( $\mathrm{MIC}=0.0625 \mu \mathrm{g} \mathrm{mL}^{-1}$ ) against $C$. albicans was 4 -fold higher than that of the $\mathrm{Bi}_{2} \mathrm{O}_{3}$ bulk powder $(\mathrm{MIC}=$ $0.250 \mu \mathrm{g} \mathrm{mL}^{-1}$ ) and 2-fold higher than the model drug itraconazole $\left(\mathrm{MIC}=0.125 \mu \mathrm{g} \mathrm{mL}^{-1}\right)$. Several studies are also available on the coating of titanium implants using $\mathrm{Bi}$ in its crystalline form or Bi-derived compounds to render implantable devices with antibacterial properties. ${ }^{298-300,302}$ For example, using a live and dead fluorescence staining technique, Gosau et al. ${ }^{298}$ demonstrated the better antimicrobial activity of Bi-coated implants against S. epidermidis when compared to silver or copper-coated implants, suggesting that Bi can be used as a new coating material for preventing biofilm formation on dental implants.

The utilization of $\mathrm{Bi}$ for antibacterial effects reveals that it has great importance in the future development of NP-based and implant-based therapeutics, not only for antimicrobial treatment, but also as a prevention measure to avoid infection due to treatment. This field seems to be in its infancy, where studies have mainly focused on the proof-of-concept demonstrations of the in vitro antimicrobial activity of the developed platforms. In this regard, it is urgent to progress to more in vivo preclinical studies where the antimicrobial properties of Bi-based materials may be practically tested. It is anticipated that Bi-based antimicrobial composites may offer potential antimicrobial activity against many pathogens with activities similar or superior to products under clinical trial or already commercially available metal-based nanocomposites, such as silver (Acticoat $\left.{ }^{\mathrm{TM}}\right)^{303}$ and $\mathrm{Au}$ (Auranofin) ${ }^{304}$ nanocomposites.

\section{Drug delivery and targeting by bismuth-containing nanomedicines}

The rapid growth of medicine has stimulated the development of new approaches for drug delivery by which the introduction of therapeutic substances is improved with respect to their efficiency and safety through the control of the rate and place of drug release in the body for various diseases, such as cancer and bacterial infection. ${ }^{11,305,306}$ From a technological point of view, the synthesis of new drug molecules is expensive and time-consuming, therefore scientists prefer to work on the formulation design of available drug molecules to introduce new methods of making them more accessible to the human body in an efficient manner. Nanoscale particles generated by organic and inorganic molecules have been widely investigated for this purpose in different ways. ${ }^{307}$ For instance, BiNPs stabilized by polyvinylpyrrolidone (PVP) have been found to assist in the NIR light-responsive release of metformin, an anti-diabetic drug, from microneedles (MNs) coated with lauric acid (LA) as a thermally-responsive material. ${ }^{308}$ BiNPs were the part of the formulation that induced the conversion of NIR light to heat, in order to melt the MNs and induce drug release. Transdermally administered MNs, compared to subcutaneous (SC) injection, remarkably induced the hypoglycemic effect in vivo for the treatment of diabetes.

One of the main challenges of BiNPs in drug delivery is the small space in their structure for the loading of therapeutic agents. Researchers have focused on developing novel BiNPs to improve their capacity for carrying drug molecules. ${ }^{81,261,309}$ In this respect, $\mathrm{Bi}_{2} \mathrm{~S}_{3}$ nanourchins $\left(\mathrm{Bi}_{2} \mathrm{~S}_{3}-\mathrm{PEG} \mathrm{NUs}\right)$ with a highly porous structure were fabricated using $\mathrm{Bi}_{2} \mathrm{O}_{3}$ nanospheres (as the Bi precursor), and then, these were PEGylated for enhanced stability. ${ }^{236}$ To fabricate the particles, $\mathrm{Bi}\left(\mathrm{NO}_{3}\right)_{3} \cdot 5 \mathrm{H}_{2} \mathrm{O}$ was completely dissolved in $\mathrm{HNO}_{3}$ solution, and then, $\mathrm{NaOH}, \mathrm{PVP}$ and EG were added into the mixture, which was then heated at $150{ }^{\circ} \mathrm{C}$ for $3 \mathrm{~h} . \mathrm{Bi}_{2} \mathrm{O}_{3}$ nanospheres were obtained after cooling the mixture down and centrifuging the sample. The $\mathrm{Bi}_{2} \mathrm{O}_{3}$ nanospheres were dispersed in a solution of thioacetamide and heated at $150{ }^{\circ} \mathrm{C}$ for $12 \mathrm{~h}$ to obtain black $\mathrm{Bi}_{2} \mathrm{~S}_{3}$ nanourchins. The final PEG-coated $\mathrm{Bi}_{2} \mathrm{~S}_{3}$ nanourchins were prepared by dispersing the samples in a PEG-SH solution under ultrasonication and then stirring overnight (Fig. 14a1). TEM images (Fig. 14a2) reveal their urchin-like morphology, with an average diameter of the inside of the sphere of $\sim 137 \mathrm{~nm}$. In addition, the porosity of the $\mathrm{Bi}_{2} \mathrm{~S}_{3}$ nanourchins was explored via Brunauer-EmmettTeller (BET) analysis of their nitrogen adsorption-desorption isotherms, demonstrating the higher porosity and surface area of $\mathrm{Bi}_{2} \mathrm{~S}_{3}$ nanourchins compared to $\mathrm{Bi}_{2} \mathrm{O}_{3}$ nanospheres, making them capable of higher drug loading, in this case doxorubicin (DOX), and enhanced cellular interactions, respectively. The $\mathrm{Bi}_{2} \mathrm{~S}_{3}$ nanourchins were found to be stable in water, but rapidly aggregated in physiological solutions, such as PBS, owing to an 

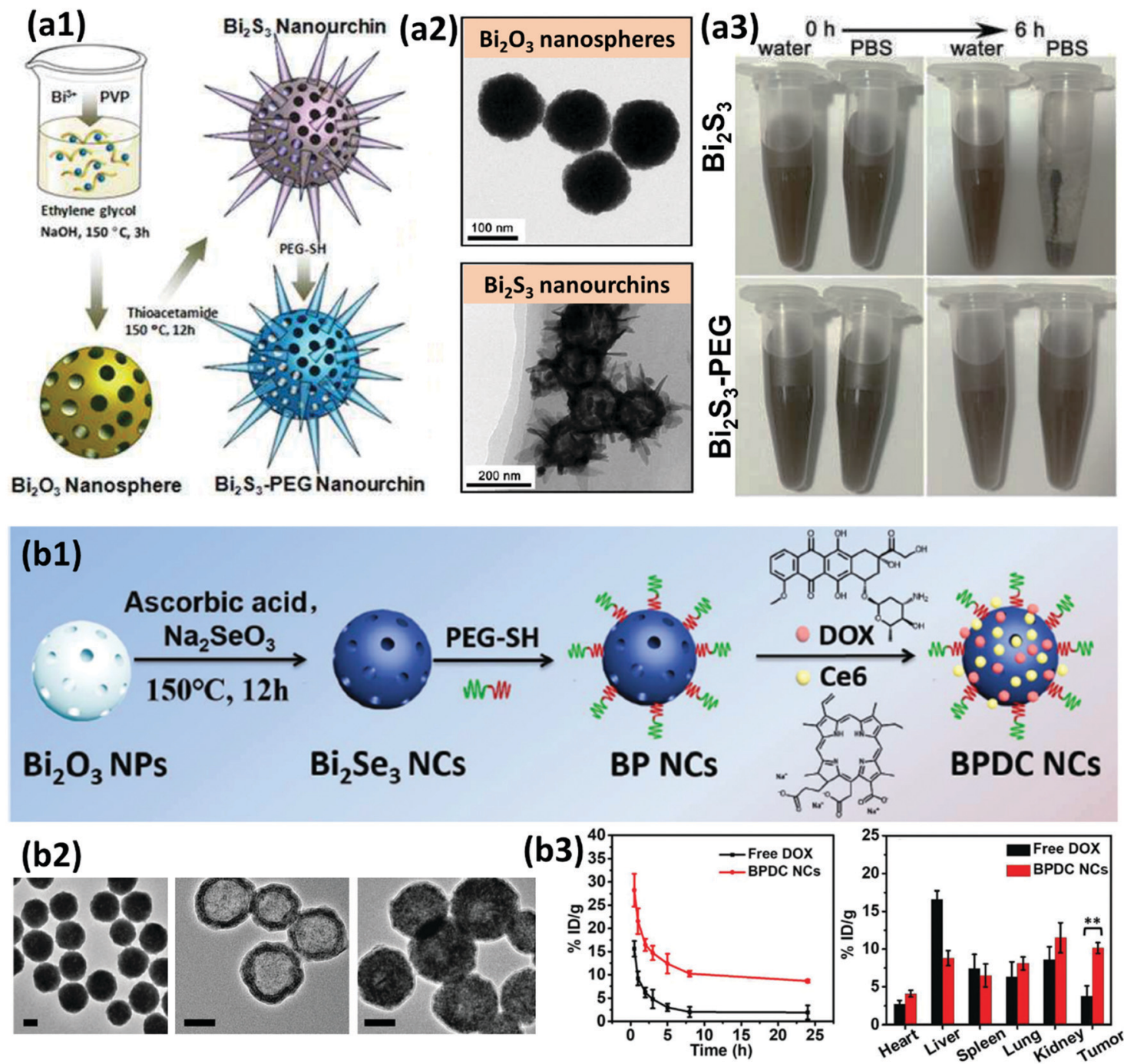

Fig. 14 (a1) A schematic representation of the synthesis of $\mathrm{Bi}_{2} \mathrm{~S}_{3}-P E G$ nanourchins. (a2) TEM images of the $\mathrm{Bi}_{2} \mathrm{O}_{3}$ nanospheres and $\mathrm{Bi}_{2} \mathrm{~S}_{3}$ nanourchins. (a3) Digital photos of $\mathrm{Bi}_{2} \mathrm{~S}_{3}$ and $\mathrm{Bi}_{2} \mathrm{~S}_{3}-\mathrm{PEG}$ nanourchins dispersed in water and PBS, showing improved stability in salt solution after the PEGylation. Adapted and reprinted with permission from ref. 236, Copyright 2016. (b1) A schematic depiction of the procedure for fabricating mesoporous hollow BPDC NCs as a multifunctional drug delivery platform. (b2) TEM images of $\mathrm{Bi}_{2} \mathrm{O}_{3} \mathrm{NPs}, \mathrm{Bi}_{2} \mathrm{Se}_{3} \mathrm{NCs}$ and BPDC NCs are shown from left to right (scale bars are $50 \mathrm{~nm}$ ). (b3) The blood concentration of DOX loaded BPDC NCs or free DOX over $24 \mathrm{~h}$ after intravenous administration to mice (left) and the biodistribution of DOX loaded BPDC NCs or free DOX $24 \mathrm{~h}$ post-injection into BALB/c mice bearing 4 T1 tumors (**p $<0.01$ between the two groups). Adapted and reprinted with permission from ref. 40, Copyright 2019.

electron screening effect (Fig. 14a3). PEG-SH was able to steadily attach onto the surface of $\mathrm{Bi}_{2} \mathrm{~S}_{3}$ through metal-S ligands to enhance the aqueous dispersity and stability of the particles in various physiological solutions. In addition to these benefits, PEGylation enhanced the blood circulation of the NPs and promoted their homing into the tumor region of $\mathrm{BALB} / \mathrm{c}$ nude mice (subcutaneously developed by the injection of HeLa epithelial adenocarcinoma cells) through enhanced permeability and retention (EPR) effect when applied for cancer therapy. PEG exhibits resistance to confiscation by the RES organs, and therefore, the leaky vascular structure and impaired lymphatic drainage of cancerous tissue presumably allow the localization of cargos of PEG-coated BiNPs within the tissue. To confirm this hypothesis, multifunctional $\mathrm{Bi}_{2} \mathrm{Se}_{3}$ @PEG nanocapsules (BP NCs) loaded with chlorin e6 (Ce6) and DOX were prepared following the procedures shown in Fig. $14 \mathrm{~b} 1 .{ }^{40} \mathrm{Bi}_{2} \mathrm{O}_{3}$ nanospheres were first formed following the same protocol used in the previous example. ${ }^{237} \mathrm{Bi}_{2} \mathrm{Se}_{3}$ NCs were subsequently prepared based on a hydrothermal process, by dispersing $\mathrm{Bi}_{2} \mathrm{O}_{3}$ nanospheres in a solution of ascorbic acid and $\mathrm{Na}_{2} \mathrm{SeO}_{4}$ in water and heating it up to $150{ }^{\circ} \mathrm{C}$ for $12 \mathrm{~h}$. PEG-SH was again used here for the PEGylation of the obtained hollow mesoporous $\mathrm{Bi}_{2} \mathrm{Se}_{3} \mathrm{NCs}$, which were subsequently loaded with Ce6 and DOX to prepare $\mathrm{Bi}_{2} \mathrm{Se}_{3} @ \mathrm{PEG} / \mathrm{DOX} / \mathrm{Ce} 6$ (BPDC) NCs. The morphology of the particles in each step is shown in Fig. 14b2. Prolonged blood circulation and efficient EPR mediated accumulation of DOX in a subcutaneously inoculated 4T1 mammary gland breast cancer cell model was detectable when the drug loaded BPDC NCs were administered (Fig. 14b3). Similar to this study, DOX and Ce6 co-loading within PEGylated star-like $\mathrm{Bi}_{2} \mathrm{~S}_{3}$ NPs, which were 
prepared via the thioacetamide treatment of $\mathrm{Bi}_{2} \mathrm{O}_{3}$ NPs, was also reported. ${ }^{49}$ The hydrodynamic size of the drug encapsulated particles was $272.8 \mathrm{~nm}$ and their negative surface charge was expected to benefit their long blood circulation. ${ }^{310}$

In addition to the above strategies offered for drug encapsulation within the BiNPs, direct surface conjugation of the drug molecule to the particles, ${ }^{311}$ loading of the therapeutics into a mesoporous shell surrounded around the BiNP core, ${ }^{312}$ as well as combined loading of the drug and BiNPs into a nanosized polymeric hydrogel ${ }^{12}$ have been investigated. For example, Bogusz et al. ${ }^{311}$ fabricated $\alpha-\mathrm{Bi}_{2} \mathrm{O}_{3}$ NPs that were linked with methotrexate (MTX) through (3-aminopropyl) trimethoxysilane (APTMS) in a core-shell structure. The $\alpha-\mathrm{Bi}_{2} \mathrm{O}_{3}$ NPs were first coated with APTMS and then conjugated to MTX through amidation between the amine moieties on the shell and the carboxylic acid groups of MTX molecules. MTX was released from the surface of the NPs via cleavage of the amide bond at low $\mathrm{pH}$. As for the second approach, mesoporous silicacoated $\mathrm{Bi}_{2} \mathrm{~S}_{3}\left(\mathrm{Bi}_{2} \mathrm{~S}_{3} @ \mathrm{MSN}\right.$; Fig. 15a1) NPs were reported for the encapsulation and protection of DOX. ${ }^{313}$ Interestingly, NIRresponsive drug release was observed from the nanocomposite, as a laser power density of $1 \mathrm{~W} \mathrm{~cm}^{-2}$ induced the release

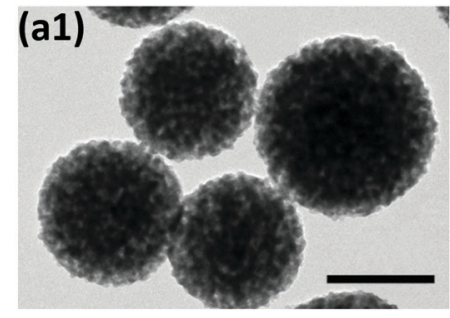

(a3)

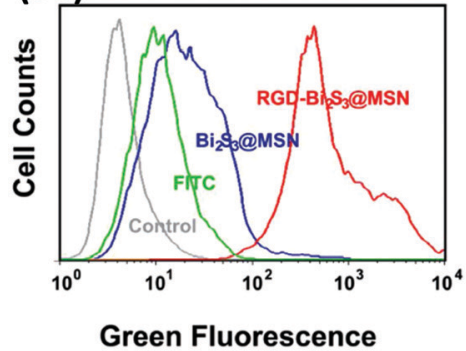

Green Fluorescence
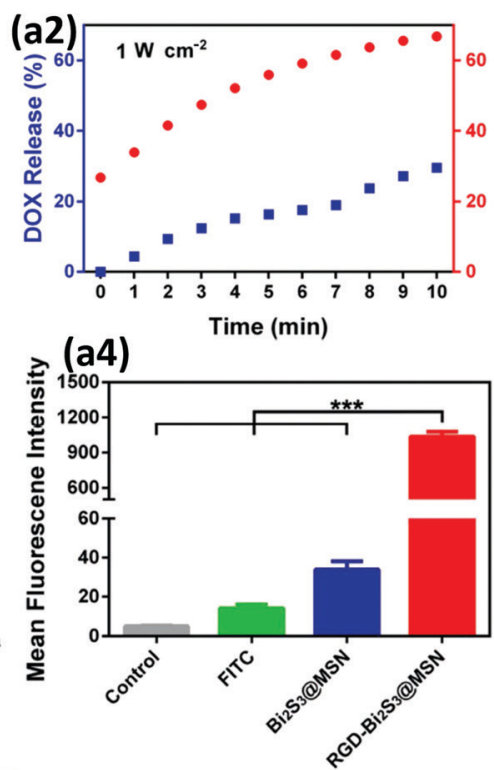

(c)

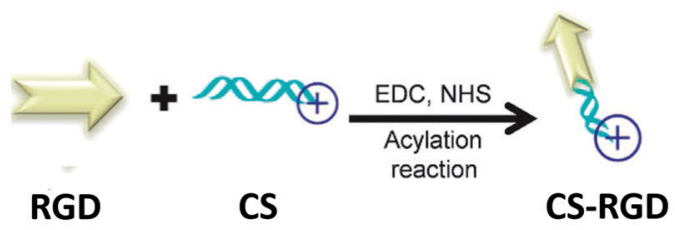

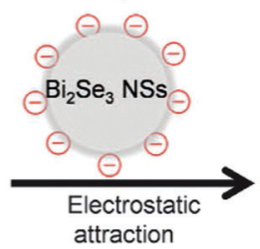

attraction (b)
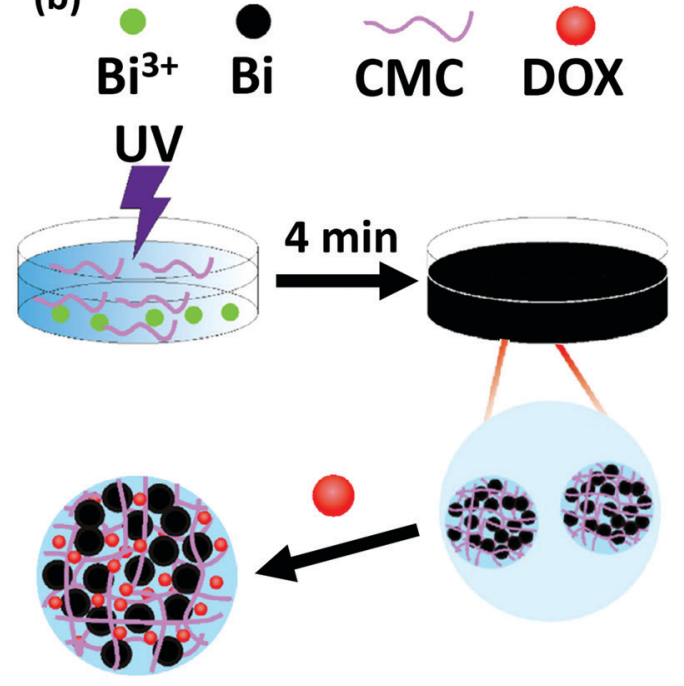

(e)

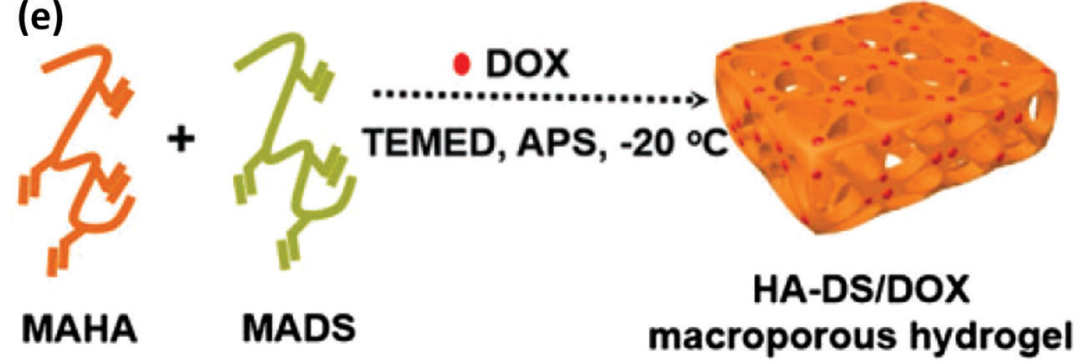

(d)

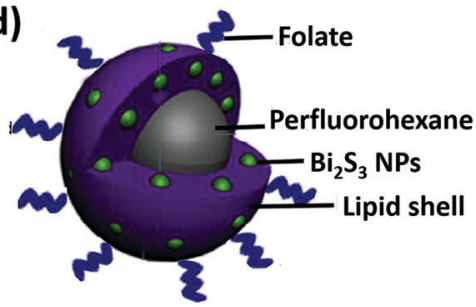

$\mathrm{Bi}_{2} \mathrm{Se}_{3}-\mathrm{CS}-\mathrm{RGD}$

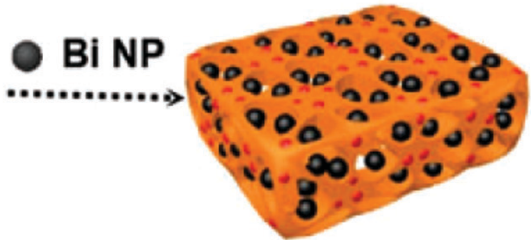

HA-DS/DOX/Bi macroporous hydrogel

Fig. 15 (a1) A TEM image of $\mathrm{Bi}_{2} \mathrm{~S}_{3} \mathrm{QMSN}$. Scale bar is $100 \mathrm{~nm}$. (a2) The drug release profile during NIR laser irradiation for $10 \mathrm{~min}$. The drug was not released without applying a NIR laser (data not shown). (a3) Flow cytometry analysis of the in vitro active targeting effect of RGD-Bi $2 \mathrm{~S}_{3} @ M \mathrm{MNN}$ in UMR-106 cells stained by free FITC, FITC- $\mathrm{Bi}_{2} \mathrm{~S}_{3} @ \mathrm{MSN}$, and FITC-RGD- $\mathrm{Bi}_{2} \mathrm{~S}_{3} \mathrm{QMSN}$. (a4) The quantitative measurement of the mean fluorescence intensity of different groups from (a3). Adapted and reprinted with permission from ref. 312, Copyright 2018. (b) The synthesis process of DOX/Bi@CMC. Adapted and reprinted with permission from ref. 12 , Copyright 2019. (c) A schematic depiction of $\mathrm{Bi}_{2} \mathrm{Se}_{3}-\mathrm{CS}-\mathrm{RGD}$ synthesis as a targeted theranostic nanomedicine, proposed for in vivo imaging-guided cancer RT. Reprinted with permission from ref. 314, Copyright 2017. (d) A schematic illustration of the final design of folate conjugated perfluorohexane core-lipid shell particles carrying $\mathrm{Bi}_{2} \mathrm{~S}_{3} \mathrm{NPs}$ in the lipid compartment. Reprinted with permission from ref. 39 , Copyright 2016. (e) A schematic illustration of preparing HA-DS hydrogel loaded with DOX and BiNPs. Reprinted with permission from ref. 315, Copyright 2018. 
of $30 \%$ of the drug from the NPs (Fig. 15a2). This feature is highly desirable for tumor therapy, since NIR laser irradiation is only applied at the site of the tumor during treatment and the controllable local release of DOX would therefore efficiently reduce the side effects experienced by normal surrounding tissues. In addition, an arginine-glycine-aspartic acid (RGD) peptide was covalently conjugated on the surface of the nanocomposite to endow the carrier with active targeting and uptake by UMR-106 epithelial cancer cells (Fig. 15a3). Similar to this study, successful active targeting of the tumor cells was reported using LyP-1 peptide conjugated PEG-Bi ${ }_{2} \mathrm{~S}_{3} \mathrm{NPs},{ }^{210}$ Trastuzumab (Tam) conjugated $\mathrm{Bi}_{2} \mathrm{~S}_{3} @$ @MN core-shell NPs, ${ }^{313}$ chitosan (CS)-RGD modified $\mathrm{Bi}_{2} \mathrm{Se}_{3}$ nanosheets (Fig. 15c), ${ }^{314}$ and folate conjugated perfluorohexane core-lipid shell particles carrying $\mathrm{Bi}_{2} \mathrm{~S}_{3}$ NPs in the lipid shell (Fig. 15d). ${ }^{39}$ As an example of the last approach, which is the combined loading of the drug and BiNPs into a nanosized polymeric hydrogel, Xuan et $a{ }^{12}{ }^{12}$ used a combined solution of carboxymethyl cellulose (CMC) and Bi citrate (BC) as initial safe materials to prepare a BiNP imbedded nanohydrogel (Bi@CMC; Fig. 15b). CMC was first dissolved in water and then combined with an aqueous solution of BC-containing ammonia. The mixture was then diluted with water and the $\mathrm{pH}$ was adjusted to $7.0 \mathrm{using} 1 \mathrm{M}$ HCl. Next, $300 \mathrm{~W}$ UV lamp irradiation was applied for 4 min to obtain and collect Bi@CMC via centrifugation. The loading of DOX was achieved by stirring $40 \mu \mathrm{g}$ of DOX and $1 \mathrm{mg}$ of $\mathrm{Bi}$ @CMC in $5 \mathrm{~mL}$ of PBS for $12 \mathrm{~h}$ in the dark. In addition to the loading of the BiNPs into the matrix of the nanohydrogels, their incorporation within injectable bulk polymeric hydrogels has also been investigated for cancer therapy. ${ }^{315}$ This work was performed to achieve the sequential delivery of BiNPs and DOX for a synergistic anticancer effect via combined X-ray radio- and chemo-therapy (Fig. 15e). A hyaluronic acid-dextran sulfate (HA-DS) macroporous hydrogel was prepared through the redox-initiated polymerization of methacrylated hyaluronic acid (MAHA) and methacrylated dextran sulfate (MADS) in PBS under frozen conditions. First, $1 \mathrm{~mL}$ of tetramethylethylenediamine (TEMED), $0.5 \mathrm{~mL}$ of ammonium persulfate (APS), and $6.5 \mathrm{~mL}$ of PBS were added to a solution containing $4 \mathrm{~mL}$ of MADS (50 $\mathrm{mg} \mathrm{mL}^{-1}$ in PBS) and $8 \mathrm{~mL}$ of MAHA $\left(50 \mathrm{mg} \mathrm{mL}^{-1}\right.$ in PBS). The mixture was then incubated for $17 \mathrm{~h}$ at $-20{ }^{\circ} \mathrm{C}$ before heating to room temperature to remove ice crystals and subsequent washing with PBS for $0.5 \mathrm{~h}$. DOX loading was performed by adding a PBS solution of the drug solution into a mixture of MAHA, MADS, TEMED, and APS before the cooling step. BiNPs were encapsulated by rehydration of the dried hydrogel with the solution of BiNPs. The loading efficiencies of the drug and BiNPs into the HA-DS hydrogel were found to be $83.8 \%$ and $85.7 \%$, respectively. The hydrogel offered sequential delivery profiles for co-loaded BiNPs and DOX. BiNPs were released completely over $24 \mathrm{~h}$, while the release profile of DOX was very slow over the first $24 \mathrm{~h}$ and continued over one week, resulting in a survival rate of $100 \%$ in mice through a synergistic anticancer effect on a 4T1 mammary gland breast cancer tumor-bearing mouse model mediated by combined X-ray radio- and chemotherapy.
Taken together, these findings on the drug delivery and targeting potential of BiNPs provide insights for broadening the investigation of these carriers in precision medicine. This is mainly due to the possibility of combining these assets with multi-modal bioimaging and BiNP-mediated PTT, which are comprehensively highlighted in the coming sections.

\section{Contrast-enhanced diagnostic imaging using BiNPs}

The contrast of the image is the main criterion for defining the sensitivity of a diagnostic imaging method. In general, higher contrast efficacy is urgently needed to reduce the side effects caused by the high dose administration of contrast agents in clinical imaging. ${ }^{316-319}$ BiNPs have demonstrated high potential as a powerful contrast agent for different imaging modalities. ${ }^{38,54,236,320}$ As a result of the presence of $\mathrm{Bi}$, these versatile NPs have recently been employed for dual-modal and multi-modal imaging guided therapies for precise diagnosis and monitoring of treatment process. Scientists are currently shifting from single imaging modality, which usually cannot meet favorable diagnostic requirements, to the combination of different accurate imaging modalities using a single formulation of BiNPs, implying that they can act as novel contrast agents (CAs) and move from the bench to the bedside in the near future. ${ }^{212}$

In this section, a comprehensive overview of the recent advances in the area of BiNP-based imaging is provided to demonstrate the feasibility of creating contrast-enhanced multi-diagnostics using these NPs.

\subsection{Computed tomography (CT) imaging using BiNPs}

CT imaging is considered to be one of the most popular imaging modalities owing to the possibility of deep-tissue imaging with high resolution. ${ }^{321} \mathrm{Bi}$ possesses a higher K-edge value $(90.5 \mathrm{keV})$ and larger X-ray attenuation coefficient $\left(5.74 \mathrm{~cm}^{2} \mathrm{~kg}^{-1}\right.$ at $\left.100 \mathrm{keV}\right)$ than other X-ray contrast agents, such as iodine (I), barium (Ba), gadolinium (Gd), ytterbium $(\mathrm{Yb})$, tantalum $(\mathrm{Ta})$, tungsten $(\mathrm{W})$, and Au, making it an excellent CA for CT imaging. ${ }^{322,323}$ Furthermore, the low price and high biosafety of $\mathrm{Bi}^{198}$ compared to other elements used for the formation of NP-based X-ray contrast agents, have added more value to its application as a CA. For example, Bi is nearly 2000 times cheaper per mole and has double the terrestrial abundance of $\mathrm{Au},{ }^{120}$ which are attractive criteria from an economical point of view. Moreover, Bi-containing medicines have been applied clinically for more than three centuries at relatively high doses, ${ }^{324}$ thus their biosafety has been more explored compared to other heavy metal-based nanoagents such as AuNPs. Evaluation of the CT contrast performance of Tween 20 coated $\mathrm{Bi}_{2} \mathrm{~S}_{3}$ nanorods (NRs; Fig. 16a1) revealed that the Hounsfield units (HU) slope of the particles is 39.36, which is much higher than that of iopromide, which has a $\mathrm{HU}$ of 16.38. ${ }^{38}$ TEM images of the $\mathrm{Bi}_{2} \mathrm{~S}_{3}$ NRs show a uniform size with a diameter of around $10 \mathrm{~nm}$ and a length of up to $50 \mathrm{~nm}$ (Fig. 16a2). The size was increased to about $90 \mathrm{~nm}$ after 

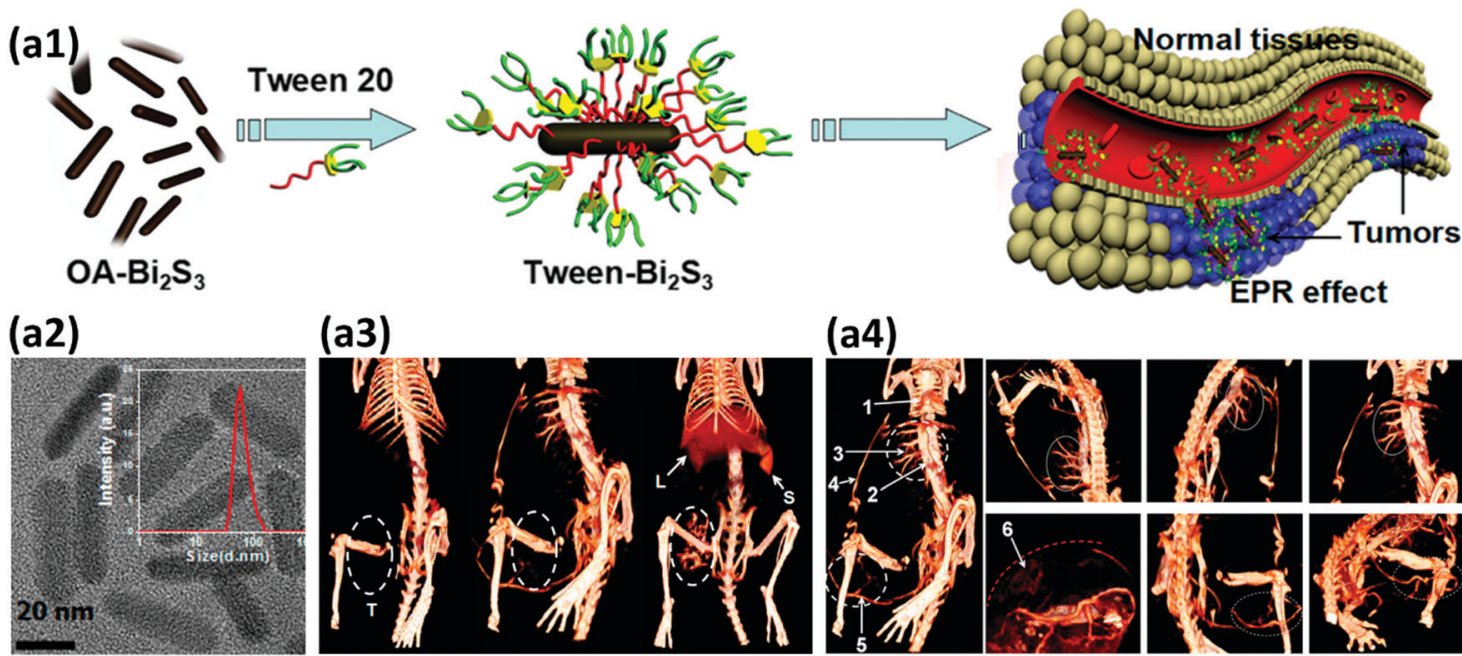

Tween- $\mathrm{Bi}_{2} \mathrm{~S}_{3}$

EPR effect

(a4)
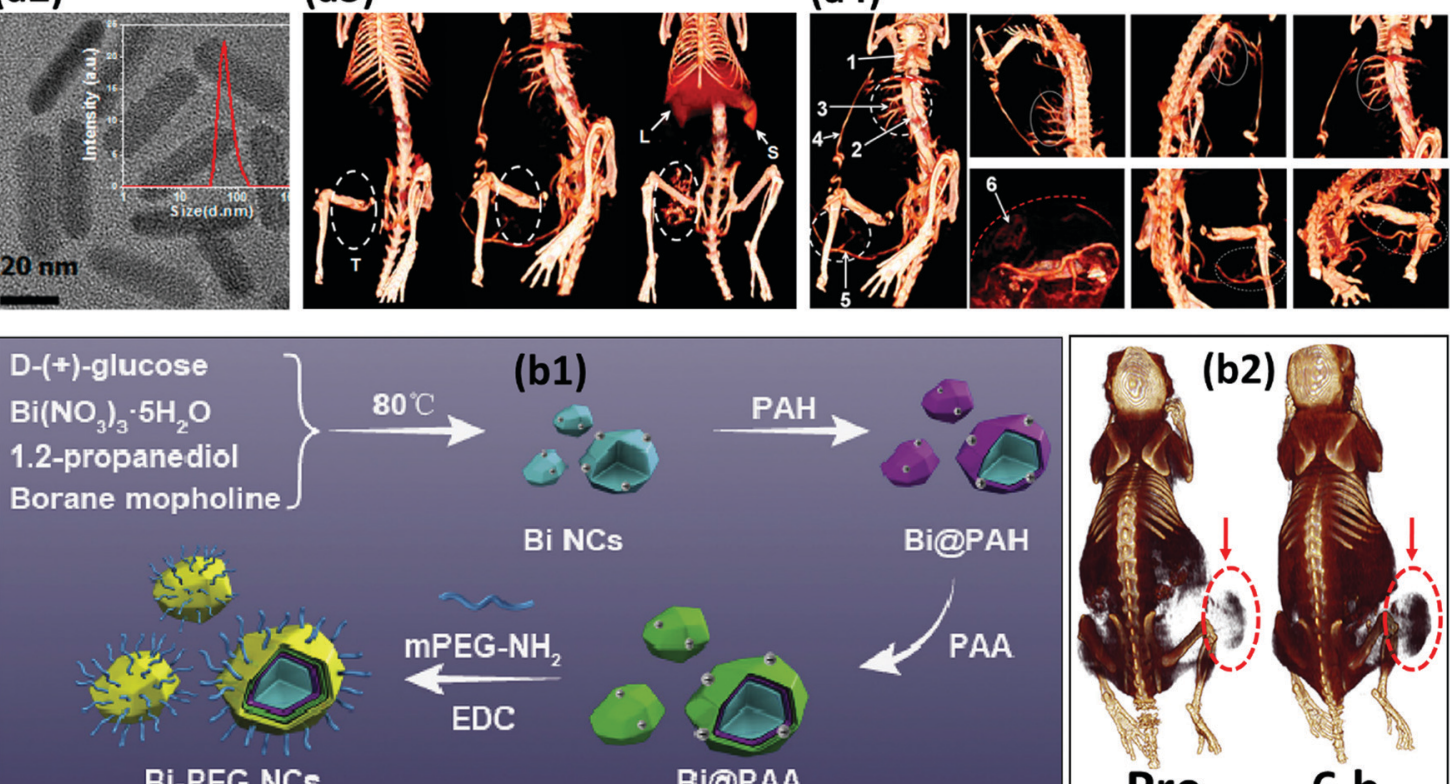

(b1)

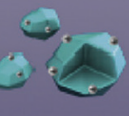

PAH

Bi NCs

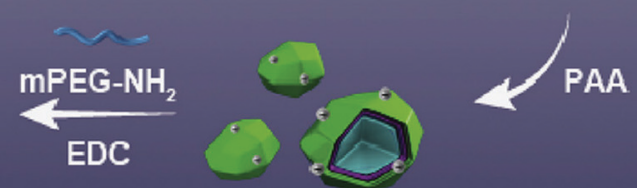

Bi-PEG NCs

BI@PAA
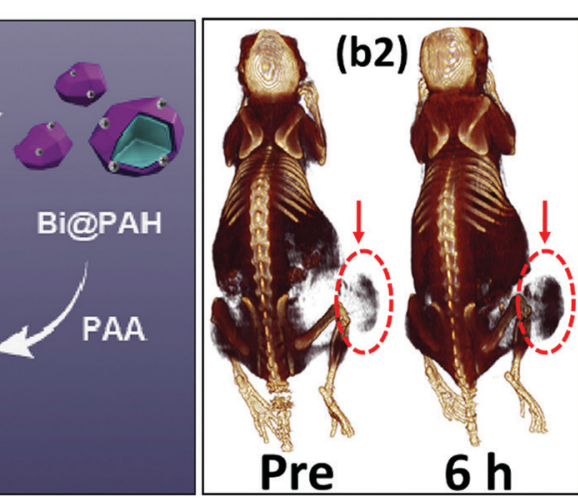

Fig. 16 (a1) A schematic illustration of the design of Tween $-\mathrm{Bi}_{2} \mathrm{~S}_{3}$ NRs for EPR-based CT imaging. (a2) TEM images of $\mathrm{Bi}_{2} \mathrm{~S}_{3} \mathrm{NRs}$, as well as the size distribution of Tween- $\mathrm{Bi}_{2} \mathrm{~S}_{3}$ NRs measured via dynamic light scattering (DLS). (a3) In vivo X-ray CT imaging in $4 \mathrm{T1}$ tumor-bearing mice. The left image shows the cancer tissue in the white circle before injection. The middle image demonstrates the tumor area in the white circle 5 min post IV injection of the Tween- $\mathrm{Bi}_{2} \mathrm{~S}_{3}$ NRs. The right image shows the cancer tissue of the mice $3 \mathrm{~h}$ post-intravenous injection of the Tween- $\mathrm{Bi}_{2} \mathrm{~S}_{3} \mathrm{NRs}$. Liver (L), spleen (S), and tumor (T) are shown in the images. (a4) CT images of the mice 5 min post IV injection of the Tween- $\mathrm{Bi}_{2} \mathrm{~S}_{3} \mathrm{NRs}$ to highlight the vascular structures. The numbers 1 to 6 correspond to the heart, vena cava, pulmonary vein plexus, vena epigastrica, tumor surface vascularity (from the abdominal venous branch), and tumor microvessels (TMV), respectively. Adapted and reprinted with permission from ref. 38 Copyright 2015. (b1) A schematic depiction of the synthesis of the Bi-PEG NCs. (b2) 3D in vivo CT images of the tumor-bearing mice before (Pre) and $6 \mathrm{~h}$ after the IV injection of the Bi-PEG NCs $\left(5.0 \mathrm{mg} \mathrm{mL}^{-1}, 200 \mu \mathrm{L}\right)$. Adapted and reprinted with permission from ref. 213, Copyright 2017.

modification and formation of a condensed Tween layer on the surface, as illustrated in the inset of Fig. 16a2. CT imaging of tumor-bearing mice revealed a collection of remarkable CT contrast signals in the tumor $3 \mathrm{~h}$ post injection, confirming the sufficient accumulation of NRs at the site of interest (Fig. 16a3). In addition, the vasculature was hyperattenuating 5 min post intravenous (IV) injection of $\mathrm{Bi}_{2} \mathrm{~S}_{3}$ NRs and led to clear angiography with contrast filling of the heart, vena cava, vena epigastrica, pulmonary vein plexus, tumor surface vascularity, and tumor microvessels (Fig. 16a4), demonstrating the great potential for the use of $\mathrm{Bi}_{2} \mathrm{~S}_{3}$ NRs in the diagnosis of vascular disease and tumor imaging. The surface coating of the NRs with Tween 20 favored an efficient EPR effect and long residence time of the particles in the tumor for CT imaging. PEG-coated $\mathrm{Bi}_{2} \mathrm{~S}_{3}$ nanodots have also demonstrated favorable accumulation in the tumor tissue. ${ }^{325}$ Nevertheless, compared to the previous example, ultra-small size of these nanodots would render them with the benefits of efficient penetration into a deeper area of the tumor followed by efficient clearance from the body after imaging.

Double or multiple-component BiNPs, ${ }^{326}$ similar to the above examples, confine the amount of $\mathrm{Bi}$ in the particles, and as a consequence, higher concentrations of the NPs are needed in order to achieve sufficient CT contrast. It is demonstrated that the Bi content in the $\mathrm{Bi}_{2} \mathrm{~S}_{3}$ NPs is nearly half that of pure BiNPs of identical size. ${ }^{327}$ Therefore, the synthesis of pure metallic BiNPs has been of recent interest as the most effective way of promoting CT imaging potential. ${ }^{213}$ Li et al. ${ }^{213}$ developed PEGylated metallic Bi nanocrystals (Bi-PEG NCs) for the first time as a competent diagnostic agent for high-performance CT imaging. The Bi NCs were first synthesized via a chemical reduction method from readily available $\mathrm{Bi}^{(\mathrm{III})}$ nitrate in $\mathrm{D}-(+)$-glucose saturated 1,2-propanediol (PPD). Borane morpholine, dissolved in PPD, was added into the above solution to activate the reaction at $80{ }^{\circ} \mathrm{C}$ under stirring, in which the mixture turned black very rapidly as an indication of the formation of the 
Bi NCs (Fig. 16b1). Since the bare Bi NCs were susceptible to aggregation and rapid oxidation when dispersed in water for one week at room temperature, they were functionalized with PEG after layer-by-layer polymeric coating, as shown in Fig. $16 b 1$. Negatively charged Bi NCs were sequentially coated with cationic poly(allylamine hydrochloride) (PAH) and anionic poly(acrylic acid) (PAA) via electrostatic adsorption, while 1-ethyl-3-(3dimethylaminopropyl)carbodiimide (EDC) was used in the last step to covalently conjugate $\mathrm{PEG}-\mathrm{NH}_{2}$ on the surface of the particles through the formation of amide groups with the carboxyl groups of PAA. The zeta potential of the bare Bi NCs increased from -10 to $+36 \mathrm{mV}$ after the fabrication of $\mathrm{Bi} @ \mathrm{PAH}$, and subsequently decreased to $-19 \mathrm{mV}$ after PAA coating. Successful layer-by-layer assembly was further confirmed via the observation of a constant but slight enhancement of the average hydrodynamic size after each coating step. The CT contrast signals were first measured by acquiring phantom images of the aqueous suspensions in vitro, and comparing them with those of high performance CT contrast agent, i.e., $\mathrm{Bi}_{2} \mathrm{Se}_{3}$ nanoplates. ${ }^{328}$ Images with a higher resolution and brightness were obtained with Bi-PEG NCs since the signal from these particles was much higher than that of the $\mathrm{Bi}_{2} \mathrm{Se}_{3}$ nanoplates. The X-ray absorption coefficient of the Bi-PEG NCs was as high as $\sim 60.3 \mathrm{HU} \mathrm{mL} \mathrm{mg}{ }^{-1}, 2.3$-fold greater than that of the $\mathrm{Bi}_{2} \mathrm{Se}_{3}$ nanoplates $\left(\sim 26.2 \mathrm{HU} \mathrm{mL} \mathrm{mg}{ }^{-1}\right)$ and 3.7-fold greater than clinically-available iopromide $\left(\sim 16.4 \mathrm{HU} \mathrm{mL} \mathrm{mg}^{-1}\right)$. To the best of our knowledge, this is one of the highest values so far reported for a CT contrast agent, when compared to $\mathrm{Bi}_{2} \mathrm{~S}_{3}$ nanorods, ${ }^{38}$ PEGylated $\mathrm{Bi}_{2} \mathrm{~S}_{3}$ nano-urchins, ${ }^{236}$ PEGylated Fe@Bi $\mathrm{S}_{3}$ NPs, ${ }^{54}$ or Au-, Pt-, W-, or Ta-based CT contrast agents. ${ }^{329-333}$ In vivo CT imaging of HeLa adenocarcinoma tumor-bearing Balb/c mice demonstrated weak CT signals in the cancerous tissue before the intravenous injection of Bi-PEG NCs $\left(5.0 \mathrm{mg} \mathrm{mL}{ }^{-1}, 200 \mu \mathrm{L}\right)$, while EPR phenomenon and efficient passive accumulation of the particles in the tumor resulted in a gradual increase in the signal to its maximum level $6 \mathrm{~h}$ after injection (Fig. 16b2). The signal then decreased slowly and lasted up to $24 \mathrm{~h}$.

The concept of single component BiNPs for X-ray imaging was further studied by Yang et al. ${ }^{72}$ through the fabrication of 1,2-dilauroyl-sn-glycero-3-phosphocholine (DLPC) BiNPs (denoted as Bi@DLPC NPs), with a desirable size of $47 \pm 3 \mathrm{~nm}$ (Fig. 17a). Herein, $\mathrm{Bi}^{(\mathrm{III})}$ acetate, dissolved in a mixed solution of $\mathrm{OA}$ and 1-dodecanethiol, was used as a precursor for the formation of BiNPs. The mixture was first heated at $40{ }^{\circ} \mathrm{C}$ for 30 min under vacuum, followed by a temperature increase up to $100{ }^{\circ} \mathrm{C}$ in the presence of nitrogen. The monodispersed BiNPs were then rapidly obtained by treating the transparent yellow solution of the previous step at $100{ }^{\circ} \mathrm{C}$ with trioctylphosphine solution containing 1-dodecanethiol. Bi@DLPC NPs were prepared by ultrasonic treatment of the mixed BiNPs and DLPC vesicles in deionized water for $10 \mathrm{~min}$. The CT imaging properties of the Bi@DLPC NPs were found to be concentration dependent and the signal intensity, as well as HU values, remarkably increased when the concentration of the NPs increased from 0.313 to $5 \mathrm{mg} \mathrm{mL}^{-1}$ (Fig. 17b and c).

In both abovementioned examples of single-component BiNPs, the size of the NPs was bigger than $40 \mathrm{~nm}$, which is (a)

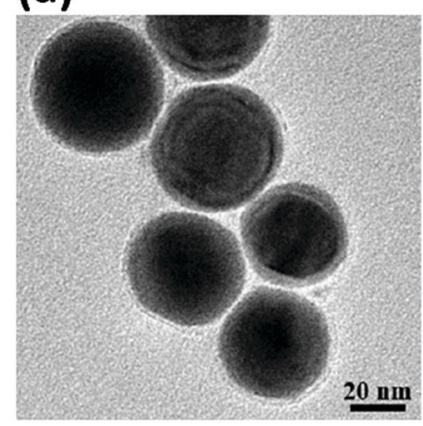

(b)
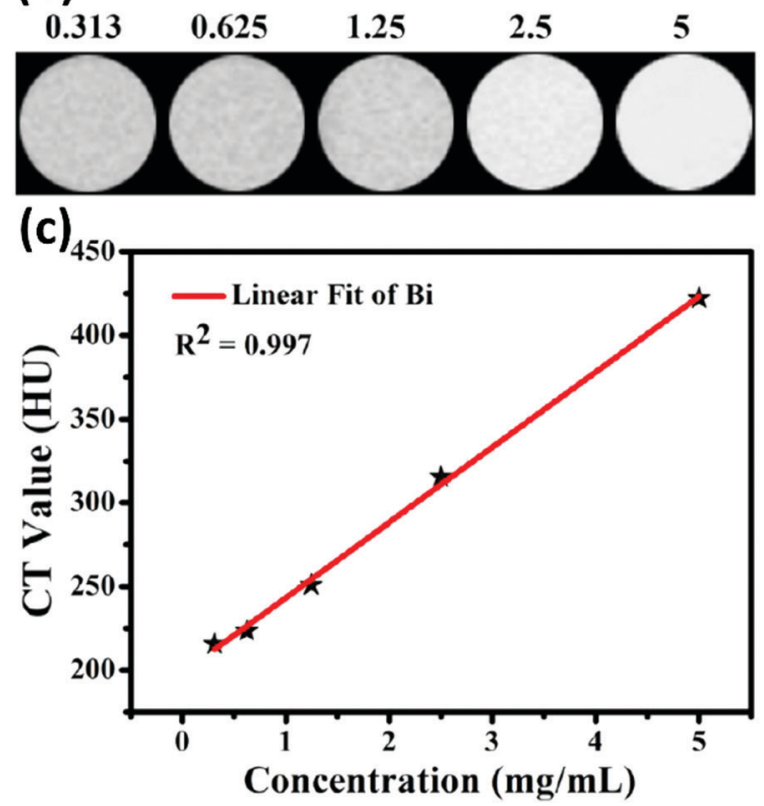

Fig. 17 (a) A TEM image of BiaDLPC NPs (left) and the corresponding EDS elemental mapping analysis. Bi (red) and phosphorus (green) were identified in the elemental analysis, confirming the successful DLPC coating outside of the BiNPs. (b) In vitro CT images and (c) the corresponding HU values of various concentrations ( $\mathrm{mg} \mathrm{mL}^{-1}$ ) of Bi@DLPC NPs. Adapted and reprinted with permission from ref. 72, Copyright 2018.

not optimal for desirable EPR-based accumulation in the tumor and subsequent non-toxic clearance from the body through the urinary system. Taking this into account, other studies have presented ultra-small Bi-PEG nanodots with a size of $c a .4 \mathrm{~nm}^{334}$ and PVP-protected Bi nanodots with a size of $c a .2 .7 \mathrm{~nm},{ }^{335}$ which might presumably work better for tumor imaging at very low concentrations due to their higher accumulation in the cancer tissue. In addition, since the size of these particles is less than the FST of the capillary walls of glomerulus, they are theoretically able to be easily cleared from the urinary system instead of their long-term accumulation in the liver and spleen.

\subsection{Photoacoustic (PA) imaging and infrared thermography (IRT) using BiNPs}

Photoacoustic imaging (PAI) is an emerging noninvasive diagnostic technology that employs the photoacoustic (PA) effect to overcome the high degree of scattering of optical 
photons in biological tissue, thereby resulting in higher spatial resolution of images obtained from living subjects. ${ }^{336} \mathrm{Com}-$ pared to other optical imaging approaches, this technique is advantageous for visualizing deeper tissues when CAs with efficient photothermal conversion are used. ${ }^{337}$ Light absorption by PA CAs induces a thermally induced pressure jump that produces ultrasonic waves, which are then received by acoustic detectors to form PA images. ${ }^{338,339}$ BiNPs are expected to play this role via their desirable photothermal conversion efficiency in the NIR window. $\mathrm{Bi}_{2} \mathrm{~S}_{3}$ nanorods ${ }^{340}$ with a size of $155.4 \pm 2.8 \mathrm{~nm}$ and $\mathrm{Bi}_{2} \mathrm{Se}_{3}$ nanosheets ${ }^{28}$ with sizes of 3 and $80 \mathrm{~nm}$ are examples of PAI using BiNPs. In the latter study, Xie et al. ${ }^{28}$ showed that BiNPs of smaller size were more promising for PAI. This could be associated with the lower scattering introduced by the smaller $\mathrm{Bi}_{2} \mathrm{Se}_{3}$ nanosheets, by which the external NIR light could be proficiently absorbed instead of being scattered. ${ }^{341}$ This led to effective photothermal conversion and higher thermal expansion that synergistically promoted the PA conversion capability of the smaller nanosheets. ${ }^{342}$ In addition to the abovementioned studies, more complex Bi-based NPs have been reported as multifunctional carriers that combine PAI with targeting, drug delivery, as well as other imaging modalities. ${ }^{43,50,343,344}$ As for combined active targeting and PAI, Yu et al ${ }^{43}$ fabricated multifunctional BiNPs by dissolving $1 \mathrm{mmol}$ of $\mathrm{Bi}^{\text {(III) }}$ acetate in $5 \mathrm{~mL}$ of $\mathrm{OA}$ for $1 \mathrm{~h}$ under vacuum at $100{ }^{\circ} \mathrm{C}$ before additional mixing under dry nitrogen for $30 \mathrm{~min}$. This mixture was added to $25 \mathrm{~mL}$ of hot oleylamine (OAm) at $260{ }^{\circ} \mathrm{C}$ and the reaction was carried out for $10 \mathrm{~min}$ (Fig. 18a1). The initial size of the BiNPs was about $2 \mathrm{~nm}$ (Fig. 18a2) and increased to $c a .12 \mathrm{~nm}$ after surface coating with PEG and further conjugation of the LyP-1 peptide. The final formulation, termed as Bi-LyP-1 NPs, exhibited excellent stability in different physiological solutions and high accumulation in a 4T1 mammary gland breast cancer tumor model (developed in the fat pad of the right legs of mice) because of the tumor-homing LyP-1 peptide on their surface. Light absorption in the second near-infrared (NIR-II) window also rendered them with the ability of diagnostic imaging through a PA modality. The PAI of tumor bearing mice that were intratumorally (i.t.) injected with Bi-LyP-1 NPs $\left(6 \mathrm{mg} \mathrm{mL} \mathrm{m}^{-1}, 30 \mu \mathrm{L}\right)$ showed a high PA signal intensity with an approximately 3.3 -fold increase when exposed to $700 \mathrm{~nm}$ laser irradiation (Fig. 18a3 and a4). The PAI of mice injected with Bi-LyP-1 NPs through an intravenous route $\left(6 \mathrm{mg} \mathrm{mL}^{-1}, 200 \mu \mathrm{L}\right)$ also demonstrated a time-dependent lightening, in which the PA signal intensity of tumors increased by $c a$. 3.6-fold at $8 \mathrm{~h}$ post-injection under $700 \mathrm{~nm}$ laser irradiation (Fig. 18a3 and a5). Similar results were obtained with 800 and $900 \mathrm{~nm}$ laser irradiation, indicating the great promise that BiNPs hold as highly competent CAs for PAI guided cancer therapy under various NIR wavelengths.

As for combined drug delivery and imaging, Dou et al. ${ }^{50}$ reported $\mathrm{Bi}_{2} \mathrm{~S}_{3}$ decorated graphene nanosheets that were functionalized with PVP (termed as $\mathrm{PVP}-\mathrm{rGO} / \mathrm{Bi}_{2} \mathrm{~S}_{3}$ ) and loaded with DOX (Fig. 18b1 and b2). The nanocomposite possessed a very high drug loading capacity of nearly $\sim 500 \%$ for DOX, which was far beyond the loading values so far reported for nanosized chalcogenides. This was mainly attributed to the large surface area of $\mathrm{rGO}$ and the strong $\pi-\pi$ stacking interaction between the drug and rGO. Dual $\mathrm{pH}-$ and NIR-responsive drug release along with PAI (Fig. 18b3-b5) were found to be the main capabilities of this multifunctional nanocomposite. Combined drug delivery and PAI have also been reported through the synthesis of core-shell nanostructures. PEG modified mesoporous silica-coated $\mathrm{Bi} \mathrm{NCs}\left(\mathrm{Bi} @ \mathrm{mSiO}_{2}-\mathrm{PEG}\right)$ is an example in which the $\mathrm{Bi}$ core is fabricated via a chemical reducing method. $^{344}$ This nanohybrid had a final average size of $c a$. $73 \mathrm{~nm}$ and the thickness of $\mathrm{mSiO}_{2}$ was $c a .12 \mathrm{~nm}$, while evenly wrapping around the $\mathrm{Bi} \mathrm{NC}$ and carrying DOX inside its mesopores. In addition to the possibility of loading medicines into the porous structure of the $\mathrm{mSiO}_{2}$ shell, Zheng et al. ${ }^{239}$ found that $\mathrm{SiO}_{2}$ coating $\mathrm{Bi}_{2} \mathrm{~S}_{3}$ can enhance the biocompatibility and stability of the BiNPs in the harsh environments of the GI tract when used orally as a probe for GI tract imaging.

Inspired by the photothermal properties of BiNPs, IRT imaging using these nanostructures has also gained plenty of attention. IRT is the science of converting infrared energy emitted from an object to apparent temperature and presenting the result as an infrared image. PVP-protected Bi nanodots have been used for this purpose as an excellent IRT imaging contrast. ${ }^{335}$ The in vivo IRT imaging capability of PVP-Bi nanodots was explored after IV injection of the particles $\left(20 \mathrm{mg} \mathrm{kg}^{-1}\right)$ into tumor-bearing mice. After $24 \mathrm{~h}$, the tumor model, established by subcutaneous injection of a U14 squamous cell carcinoma cell line in the left axilla of Balb/c female mice, was irradiated with a $808 \mathrm{~nm}$ NIR laser $\left(1.3 \mathrm{~W} \mathrm{~cm}^{-2}\right)$, and an IR thermal camera was used for imaging and measuring the temperature change of the tumors. While the temperature of the control mice did not noticeably change, the temperature of the tumor site increased after injection of the PVP-Bi nanodots. This great result indicated that PVP-Bi nanodots might be used as an excellent IRT CA for the real-time monitoring of thermal dynamics during the PTT process.

Polydopamine (PD)/HSA/DOX coated $\mathrm{Bi}_{2} \mathrm{Se}_{3}\left(\mathrm{Bi}_{2} \mathrm{Se}_{3} @ \mathrm{PD} /\right.$ DOX/HSA) NPs have also been found to act as an excellent contrast agent for IRT imaging. ${ }^{345}$ In vivo imaging using an IRT camera revealed that all tumors inoculated by human cervix HeLa adenocarcinoma cells demonstrate a green color before irradiation (i.e., at $0 \mathrm{~s}$ ). Similarly, upon $10 \mathrm{~min}$ irradiation, the color of the free DOX and saline group barely changed. In contrast, for the groups injected with $\mathrm{Bi}_{2} \mathrm{Se}_{3} @ \mathrm{PD} / \mathrm{DOX} / \mathrm{HSA}$ ( $25 \mathrm{mg} \mathrm{kg}^{-1}$ ), the tumor turned red and became brighter upon extended irradiation. The IRT camera showed that the tumor temperature varies very little for the free DOX and saline groups (the $\Delta T$ values of the free DOX and saline groups were 4.7 and $4.2{ }^{\circ} \mathrm{C}$, respectively), whilst for the $\mathrm{Bi}_{2} \mathrm{Se}_{3} @ \mathrm{PD} / \mathrm{DOX} / \mathrm{HSA}$ group, the temperature greatly increased upon $10 \mathrm{~min}$ of laser irradiation and high-contrast real-time IRT imaging of the tumor was achieved. These examples show that intelligent designs combining different functionalities of individual NPs will make a great impact in the field of diagnostic nanomedicine.

\subsection{Magnetic resonance imaging (MRI) and ultrasonography} (US)

In addition to the intrinsic properties of BiNPs for CT, PA, and IRT imaging, their nanocomposites have also been investigated 


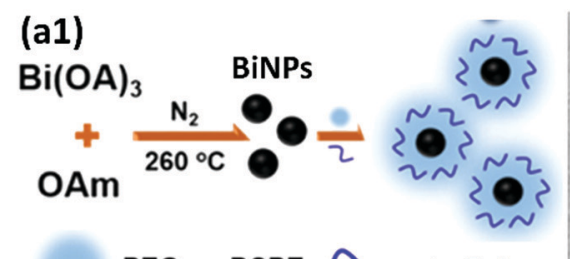

PEG $_{5000}$-DSPE $\frown$ LyP-1

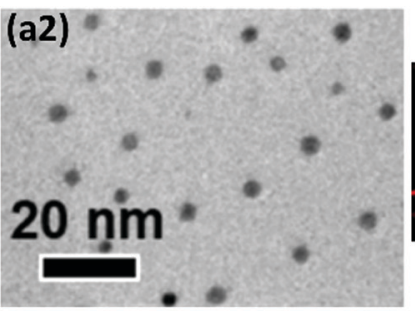

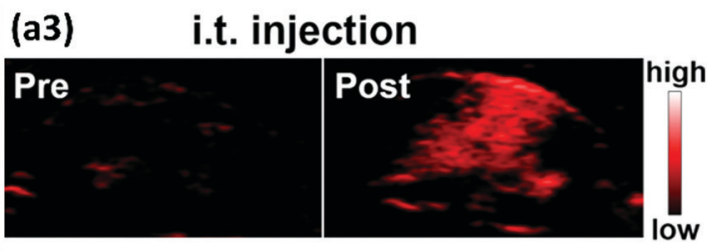

i.v. injection
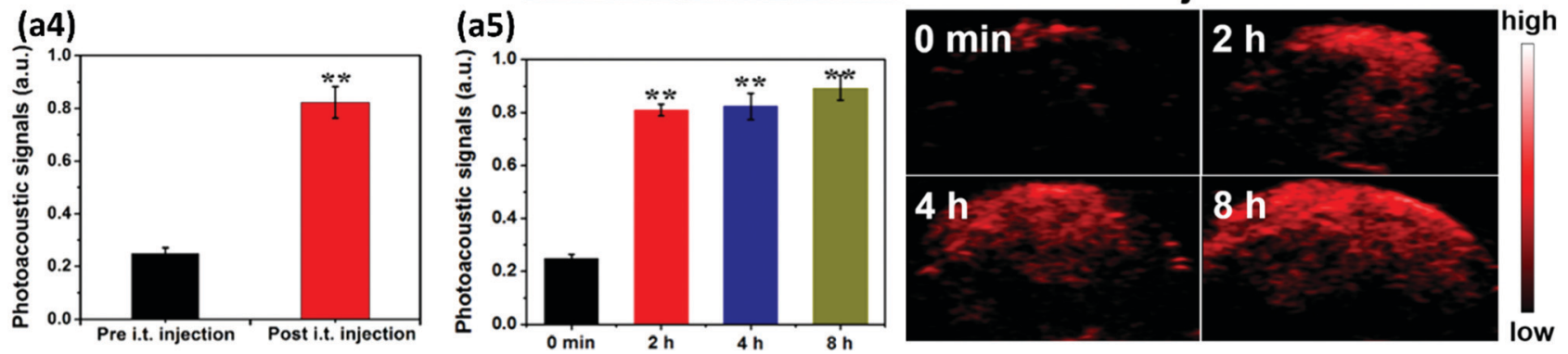

(b1)
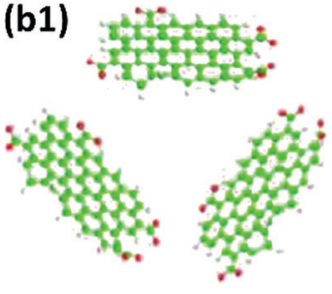

GO
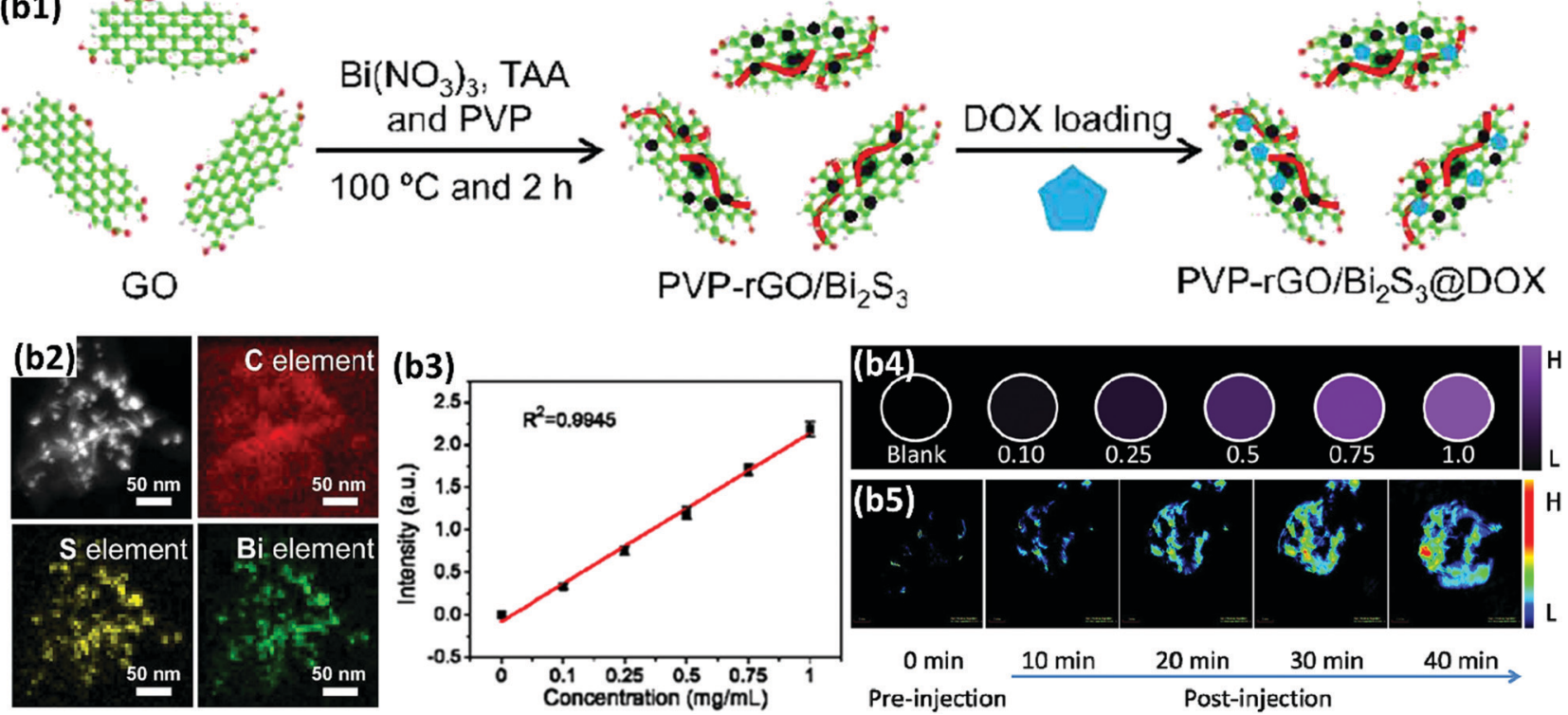

Fig. 18 (a1) A schematic diagram showing the synthetic procedure of multifunctional Bi-LyP-1 NPs. (a2) A TEM image of the BiNPs before surface coating with PEG and peptides. (a3) PA images of the tumor before and after the i.t. and intravenous administration of the Bi-LyP-1 NPs. (a4) PA signal intensities from tumors obtained before and after the i.t. administration of the Bi-LyP-1 NPs at $700 \mathrm{~nm}$. (a5) Signal intensities of tumors obtained before and after $(2,4$, and $6 \mathrm{~h})$ the intravenous administration of Bi-LyP-1 NPs at $700 \mathrm{~nm}$. Adapted and reprinted with permission from ref. 43 Copyright 2017. (b1) An illustration of the synthesis procedure of the PVP-rGO/Bi $\mathrm{S}_{3}$ nanocomposite. Thioacetamide (TAA), $\mathrm{PVP}$ and $\mathrm{Bi}\left(\mathrm{NO}_{3}\right)_{3}$ were mixed with GO and heated for $2 \mathrm{~h}$ at $100{ }^{\circ} \mathrm{C}$ to produce PVP-rGO/Bi $\mathrm{S}_{3}$. (b2) Elemental mapping of the nanocomposite (C: red; S: yellow; Bi: green), confirming the successful formation of the final formulation. (b3) A plot of PA signal versus the concentration of the nanocomposite. (b4) Photoacoustic tomography (PAT) phantom images of the aqueous dispersion of the nanocomposite with different concentrations, demonstrating its potential for PAl. (b5) The in vivo PAT imaging of a tumor-bearing mouse after the i.t. injection of the particles. It is evident that no obvious signal was detectable by PAT imaging before injection, while constant enhancement of the PAT signal was observed within a 40 min time period. Adapted and reprinted with permission from ref. 50, Copyright 2013.

for MRI and US, broadening their applications in the imaging field. MRI has been extensively applied for diagnostic imaging due to its high spatial resolution, excellent contrast difference of 3D soft tissues, and its noninvasiveness. ${ }^{346,347}$ Therefore, the integration of iron (Fe), manganese (Mn), and Gd with BiNPs enables the use of the NPs as a contrast agent for MRI. For example, due to the presence of $\mathrm{Mn}$, core-shell $\mathrm{MnSe} @ \mathrm{Bi}_{2} \mathrm{Se}_{3}$ has been applied as a MRI contrast agent. ${ }^{348}$ This nanostructure was fabricated via a partial cation exchange method, using
MnSe nanocrystals as a template in which the outer layer of Mn was used for coating with the $\mathrm{Bi}_{2} \mathrm{Se}_{3}$ through ion exchange and hydrophobic interactions before surface PEGylation. The paramagnetic MnSe core offers contrasts for both $T_{1^{-}}$and $T_{2}$-weighted MRI, whereas the presence of the $\mathrm{Bi}_{2} \mathrm{Se}_{3}$ shell means that the nanostructure can strongly absorb both X-rays and NIR light, useful for CT and IRT imaging, as well as PTT. The corresponding longitudinal relaxivity $\left(r_{1}\right)$ and transverse relaxivity $\left(r_{2}\right)$ of MnSe@Bi ${ }_{2} \mathrm{Se}_{3}-\mathrm{PEG}$ were found to be 5.44 and $27.6 \mathrm{mM}^{-1} \mathrm{~s}^{-1}$, 
respectively, comparable to the values of manganese oxide NPs. ${ }^{349}$ In vivo MRI of $4 \mathrm{~T} 1$ mammary gland breast cancer tumor-bearing mice for $T_{1}$-weighted MRI made the tumor brighter, while the $T_{2}$-weighted MRI showed negative contrast in the tumor after the injection of NPs, demonstrating the capability of $\mathrm{MnSe} @ \mathrm{Bi}_{2} \mathrm{Se}_{3}-\mathrm{PEG}$ to serve as a CA for use in both $T_{1^{-}}$and $T_{2}$-weighted MRI. Notably, the integration of $T_{1^{-}}$and $T_{2}$-weighted MR modes in a single nanoagent may potentially enhance the ability of discriminating hypointense tissue in MRI to overcome the intrinsic shortcomings of the $T_{2}$-weighted mode. ${ }^{350}$ In another example, Gd-PEG-Bi NPs were prepared for high quality MRI/CT/PAI imaging. Gd was used to make the system paramagnetic and PEGylation was used to improve the blood circulation and biocompatibility of the nanocomposite. ${ }^{48}$ The $r_{1}$ and $r_{2}$ values of the Gd-PEG-Bi NPs were found to be 4.9 and $9.6 \mathrm{mM}^{-1} \mathrm{~s}^{-1}$, respectively, which are comparable to Magnevist, a commercially available nanoagent contrast. In addition, in vivo imaging lasted for $3 \mathrm{~h}$ post-injection, which is longer than the 30 min time window of imaging using Magnevist. ${ }^{351}$ Naha et $a .^{260}$ also reported the synthesis of dextran-coated Bi-iron oxide NPs, in which the iron oxide serves as a contrast agent for $T_{2}$-weighted MRI, while Bi endows this nanocomposite with the ability to act as a CT contrast agent. Herein, it was shown that the MRI contrast decreases with the enhancement of the Bi content in the nanohybrid. Therefore, it was assumed that Bi-containing MRI CAs are not powerful enough to be used for single imaging modality, but instead effective as a dual- or multi-modality CA.

US is another modality, which has been combined with the imaging and therapeutic potentials of BiNPs through the formation of novel nanocomposites. For example, $\mathrm{Bi}_{2} \mathrm{~S}_{3}$-embedded poly(lacticco-glycolic acid) (PLGA) nanocapsules have been synthesized to achieve excellent US performance due to the presence of PLGA, which enhances the contrast of the imaging. ${ }^{209,352,353}$ The PLGA shell efficiently increases the US energy deposition, thus enhancing the HIFU therapeutic efficacy. The embedded $\mathrm{Bi}_{2} \mathrm{~S}_{3}$ NPs enabled highly improved RT. The $\mathrm{Bi}_{2} \mathrm{~S}_{3}$-PLGA NPs demonstrated good performance in real-time ultrasonography-guided HIFU therapy, determining the location of the tumor, while monitoring the treatment process.

All in all, these examples provide primary knowledge for the further investigation and design of multimodal CAs based on BiNPs as their intrinsic CT, PA and IRT imaging modalities can be combined with other imaging techniques through the fabrication of novel nanocomposites.

\section{Bi nanoplatforms for cancer therapy}

Cancer therapy can be considered a major role of BiNPs in biomedicine. To date, various types of therapeutic approaches, such as chemotherapy, PTT, RT, HIFU, photodynamic therapy (PDT), and immunotherapy, have been able to successfully enter into the clinic either as mono- or multi-therapies. Nevertheless, it is believed that there is a long way ahead for the discovery of novel anticancer agents and therapeutic strategies in laboratories for future clinical translation. Various nanomaterials have been introduced for this purpose. In this section, we describe BiNPs as one of those materials, which have demonstrated huge potential for efficient cancer treatment through both mono- and multi-therapy.

\subsection{Photothermal monotherapy of cancer by $\mathrm{Bi}$ nanoplatforms}

Thermotherapy is a type of cancer treatment in which the tumor tissue is exposed to a fatal temperature to damage proteins and structures within cells and shrink tumors. The heat tolerance of tumor tissues is lower than that of normal tissues and the damage induced by hyperthermia is irreversible because of weakened cell membranes and protein denaturation. NIR-laser mediated hyperthermia is a common method of PTT, which is believed will revolutionize the field of cancer therapy in the near future. ${ }^{37,46}$ Tumor tissue absorbs the light energy of the NIR laser and converts it to localized heat for the ablation of malignant cells. ${ }^{354-358}$ NIR-mediated PPT is desirable due to light penetration into the skin by up to several centimeters, since water, lipids, and the hemoglobin of tissues exhibit negligible absorption of light in the IR region, ranging from 700 to $1300 \mathrm{~nm}$. BiNPs have recently gained considerable interest as promising nanoagents for cancer PTT, as a result of their strong absorbance in the NIR region, high photothermal conversion efficiency, and also high biosafety. ${ }^{38,359,360}$ For example, the photothermal conversion efficiencies of $\mathrm{Bi}_{2} \mathrm{~S}_{3}$ / $\mathrm{Cu}_{2} \mathrm{~S} / \mathrm{Cu}_{3} \mathrm{BiS}_{3}$ composites, ${ }^{361} \mathrm{Bi}_{2} \mathrm{~S}_{3}-\mathrm{Au}$ heterojunction NRs, ${ }^{362}$ and hydrophilic flower-like $\mathrm{Bi}_{2} \mathrm{~S}_{3} \mathrm{NPs}^{358}$ were found to be $43.8 \%$, $51.06 \%$, and $64.3 \%$, respectively. $\mathrm{PVP}-\mathrm{Bi}_{2} \mathrm{Se}_{3}$ nanosheets with a diameter of $31.4 \mathrm{~nm}$ and a thickness of $1.7 \mathrm{~nm}$ have also been shown to have an extinction coefficient of $11.5 \mathrm{~L} \mathrm{~g}^{-1} \mathrm{~cm}$ and photothermal conversion efficiency of $34.6 \%$ at $808 \mathrm{~nm}$, which are highly desirable for the eradication of cancer tissue in vivo. ${ }^{363} \mathrm{~B}_{\mathrm{i} 2} \mathrm{~S}_{3}$ nanorods have been reported as a PTT platform $^{38}$ in which the hyperthermia-induced killing of 4T1 cancerous cells was followed by no considerable sign of pulmonary metastasis in the nanomaterial-based PTT group. In contrast, a large number of metastasis sites were found in the lungs of the control groups. This observation originated from the fact that nanomaterial-based PTT may be beneficial for triggering immunological responses against tumor cells in the body, thus contributing towards inhibiting metastasis. ${ }^{364-367}$ Since a great number of BiNPs often fail to reach cancer tissue and are rapidly eliminated from the body, cell-mediated NP-delivery has been proposed to improve the therapeutic efficiency of BiNPs through their higher transport to tumor tissue. Macrophages with innate phagocytotic capability were used for the loading and delivery of $\mathrm{B}_{\mathrm{i} 2} \mathrm{Se}_{3}$ nanosheets ${ }^{368}$ with a size of $84.0 \pm 11.3 \mathrm{~nm}$. Macrophages were shown to uptake nanosheets after $24 \mathrm{~h}$ of exposure in a dose-dependent manner (Fig. 19a1 and a2). Bi and Se biodistribution in mice revealed that for bare $\mathrm{Bi}_{2} \mathrm{Se}_{3}$ nanosheets, a large concentration of $\mathrm{Bi}$ and Se accumulates in the liver and spleen, but a small amount in an epithelial adenocarcinoma MCF-7 breast tumor model. This is probably because the tumor uptake of $\mathrm{Bi}_{2} \mathrm{Se}_{3}$ nanosheets is 


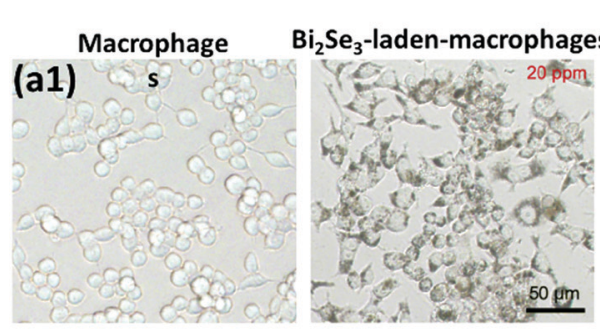

(a2)

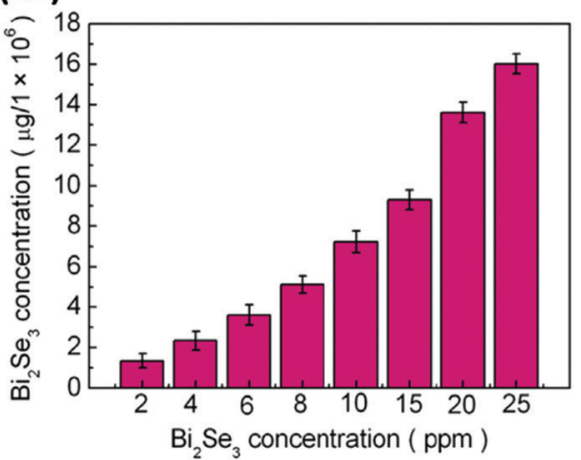

(b1)

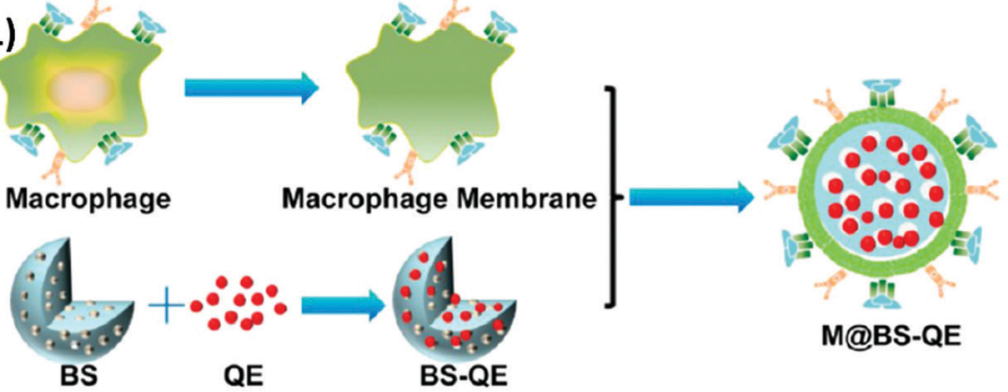

(b2)

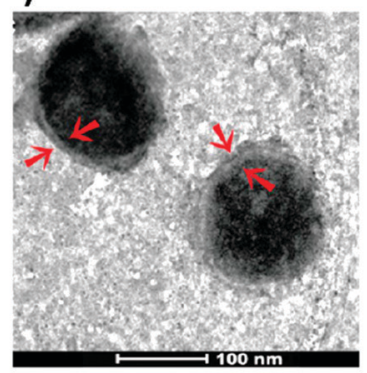

(b3)
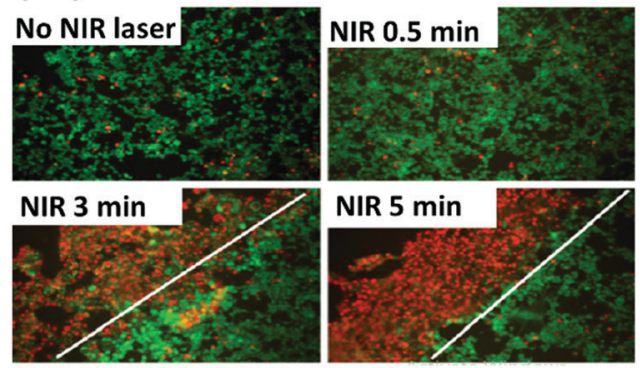

(b4)

PBS

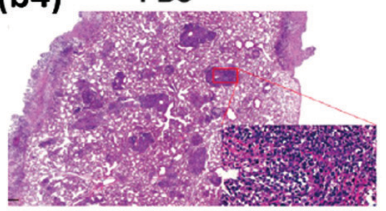

QE

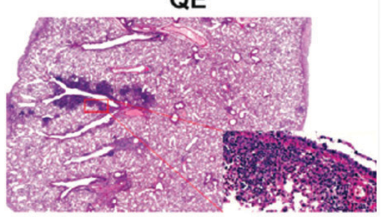

BS-QE

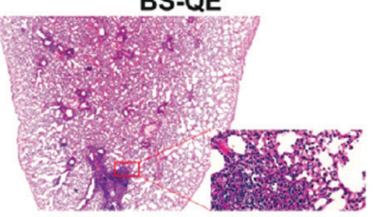

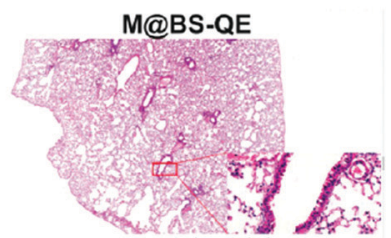

Fig. 19 (a1) The uptake of $\mathrm{Bi}_{2} \mathrm{Se}_{3}$ nanosheets by macrophages. Bright-field images of macrophages before (left) and after (right) the uptake of the $\mathrm{Bi}_{2} \mathrm{Se}_{3}$

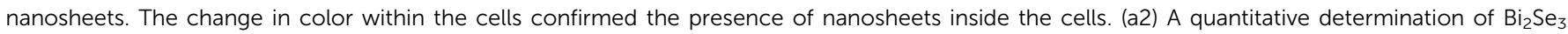
nanosheet uptake by macrophages, showing a concentration dependent trend. Adapted and reprinted with permission from ref. 368 , Copyright 2017. (b1) An illustration of the synthetic procedure for biomimetic M@BS-QE. (b2) TEM images of MaBS-QE. The cell membrane is shown between the two red arrows. (b3) An evaluation of the photothermal destruction of $4 \mathrm{~T} 1$ cells after treatment with an NIR laser at $808 \mathrm{~nm}$ in the presence of $\mathrm{Bi}_{2} \mathrm{Se}{ }_{3} \mathrm{NPs}$. (b4) Histological examination of metastatic lesions in lung tissue. The metastatic lesions are stained with dark nuclei. The PBS, free QE, and BS-QE NP treated groups exhibit plenty of dark areas in the lung, but metastatic lesions were barely visible in the M@BS-QE group, implying the promising antimetastatic performance of the M@BS-QE NPs. Adapted and reprinted with permission from ref. 372, Copyright 2018.

dependent on: (1) their low passive accumulation in the tumor, which relies on the size, shape, coating, and surface charge of the nanomaterials, and (2) the difficulty in overcoming the hypoxia-associated drug delivery barrier. ${ }^{369,370}$ In contrast, the $\mathrm{Bi}_{2} \mathrm{Se}_{3}$-laden-macrophages illustrated a much higher targeting efficiency with Bi and Se concentration in the tumor, being 3- to 4 -fold higher than those of the $\mathrm{Bi}_{2} \mathrm{Se}_{3}$ group. After PTT, the group treated with $\mathrm{Bi}_{2} \mathrm{Se}_{3}$-laden-macrophages illustrated complete tumor ablation with overall survival of $100 \%$ after up to 45 days of screening post-irradiation without tumor recurrence. In the animal group treated with the bare $\mathrm{Bi}_{2} \mathrm{Se}_{3} \mathrm{NPs}$, tumor growth was partially inhibited due to the PTT effect of $\mathrm{Bi}_{2} \mathrm{Se}_{3}$ in the first 4 days, but fast tumor growth was observed thereafter, and all the mice died within 32 days.

In addition to the delivery of BiNPs by live cells, cellmembrane-camouflaged BiNPs can be considered a perfect biomimetic nanosystem with powerful advantages that can hardly be achieved with any other type of physical or chemical surface modification. It has been proven that the $\alpha 4$ integrin of the macrophage membrane can interact with the vascular cell adhesion molecule-1 (VCAM-1) of the cancer cells, enabling active targeting of the lung metastasis of breast cancer. ${ }^{371}$ Therefore, it was assumed that the combination of BiNPs with macrophage membrane will not only provide high blood retention capacity and excellent immune escaping properties, but also active targeting ability. In this context, macrophage membrane-camouflaged quercetin (QE)-loaded hollow $\mathrm{Bi}_{2} \mathrm{Se}_{3}$ NPs (M@BS-QE; Fig. 19b1 and b2) with hyperthermia triggered drug release performance were developed for targeted inhibition and treatment of breast cancer and lung metastasis of breast cancer. ${ }^{372}$ In addition to the affinity of macrophages to the VCAM-1 of cancer cells, secretion of $\mathrm{C}-\mathrm{C}$ chemokine ligand 2 (CCL2) from breast tumors and stromata is a proven mechanism that facilitates the recruitment of $\mathrm{C}-\mathrm{C}$ chemokine receptor 2 (CCR2)-expressing macrophages to the cancer cells. Therefore, this study was aimed at benefitting from both these phenomena, using the macrophage membrane as the outer shell of the $\mathrm{Bi}_{2} \mathrm{Se}_{3}$ NPs. These NPs were found to considerably sensitize breast cancer cells to PTT (Fig. 19b3). Therefore, combining this therapeutic modality with excellent immune evading properties, CCL2/CCR2 recruitment ability, and a4/VCAM-1 identification ability of the M@BS-QE NPs led to 


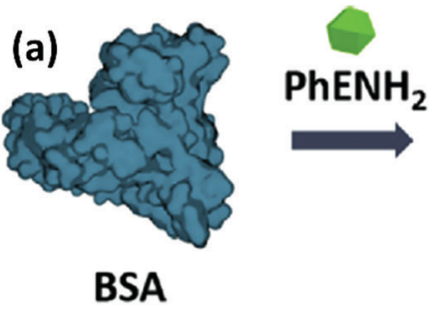

(b1)
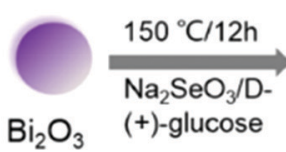

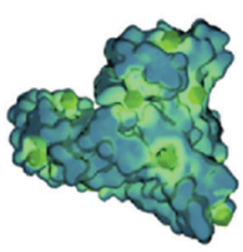

BSA-PhENH
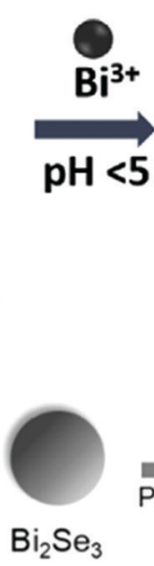

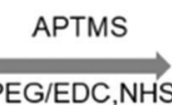

PEG/EDC,NHS

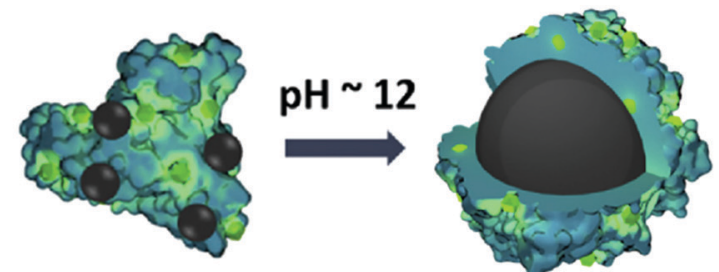

BSA-PhENH ${ }_{2}-\mathrm{Bi}^{3+} \quad \mathrm{BSA}-\mathrm{PhENH}_{2}-\mathrm{Bi}_{2} \mathrm{~S}_{3}$
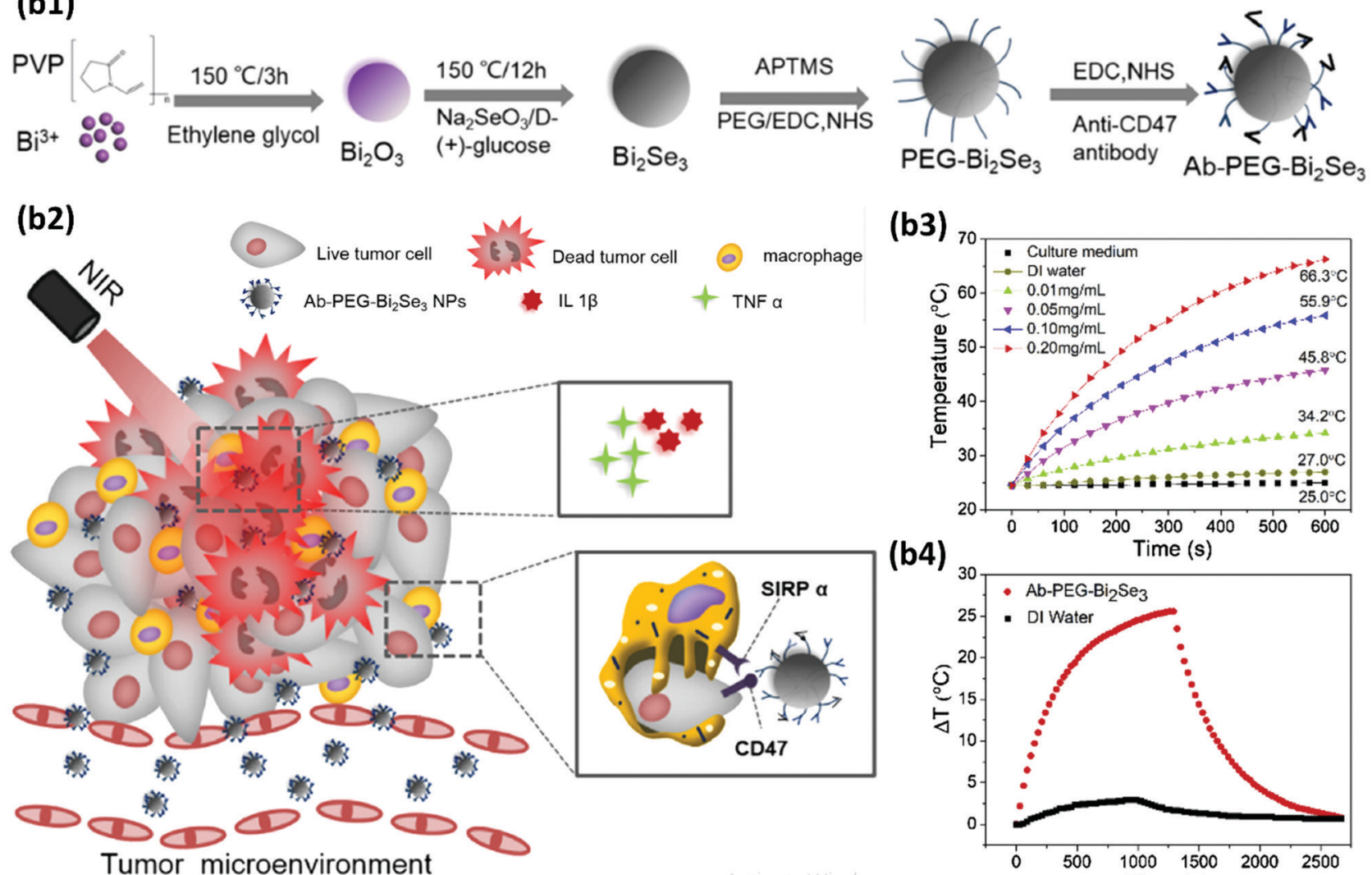

(b3)

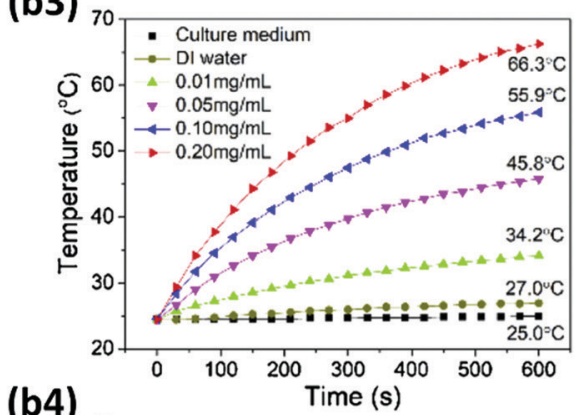

(b4)

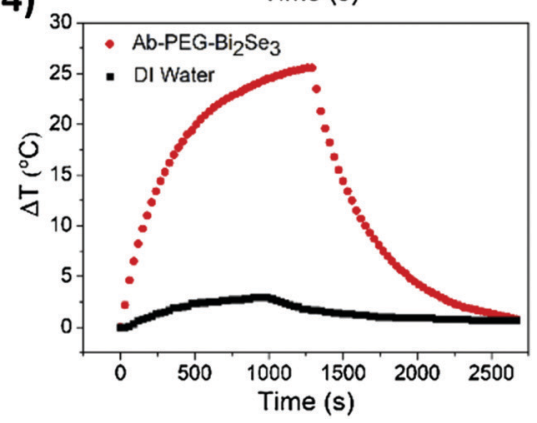

Fig. 20 (a) A schematic representation of the fabrication process of $\mathrm{BSA}-\mathrm{PhENH}_{2}-\mathrm{Bi}_{2} \mathrm{~S}_{3} \mathrm{NPs}$. Adapted and reprinted with permission from ref. 373 , Copyright 2017. (b1) A schematic depiction of the fabrication of $\mathrm{Ab}-\mathrm{PEG}-\mathrm{Bi}_{2} \mathrm{Se}_{3} \mathrm{NPs}$. (b2) A schematic illustration of the proposed anticancer mechanism of $\mathrm{Ab}-\mathrm{PEG}-\mathrm{Bi}_{2} \mathrm{Se}_{3}$ through PTT, with the inhibition of CD47-SIRP $\alpha$ interactions, secretion of stimulatory cytokines, and macrophage-mediated phagocytosis of cancer cells. (b3) The temperature of $\mathrm{Ab}-\mathrm{PEG}-\mathrm{Bi}_{2} \mathrm{Se}_{3}$ suspensions at different concentrations over a 10 min time period of irradiation with an NIR laser. (b4) A comparison of the heating and cooling curves of an Ab-PEG- $\mathrm{Bi}_{2} \mathrm{Se}_{3}$ suspension $\left(200 \mu \mathrm{g} \mathrm{mL}{ }^{-1}\right)$ and pure DI water. Adapted and reprinted with permission from ref. 374, Copyright 2019.

the outstanding suppression of breast cancer lung metastasis (Fig. 19b4).

Coating the BiNPs with biocompatible proteins/polymers and active targeting by antibodies are other strategies to improve the PPT of the cancer. ${ }^{373,374}$ In a study by Wang et al. ${ }^{373}$ protein coating was performed during the fabrication of $\mathrm{Bi}_{2} \mathrm{~S}_{3}$ NPs (Fig. 20a). An aggregation induced emission dye $\left(\mathrm{PhENH}_{2}\right)$ was first incorporated in the hydrophobic domains of BSA. Next, the BSA-PhENH complexes were coordinated with $\mathrm{Bi}^{3+}$ ions under acidic conditions before quickly increasing the $\mathrm{pH}$ to 12 to form $\mathrm{BSA}-\mathrm{PhENH}_{2}$-coated $\mathrm{Bi}_{2} \mathrm{~S}_{3} \mathrm{NPs}$. PhENH $\mathrm{H}_{2}$ was used for the fluorescence imaging of the fabricated $\mathrm{BSA}-\mathrm{PhENH}_{2}-\mathrm{Bi}_{2} \mathrm{~S}_{3} \mathrm{NPs}$, which exhibited high colloidal stability and high PTT effect upon $808 \mathrm{~nm}$ laser irradiation. Importantly, BSA not only played a significant role in improving the water dispersity of the particles and as a stabilizer of the fluorescent $\mathrm{PhENH}_{2}$ dye, but also its cysteine groups were a sulfur precursor for the further preparation of $\mathrm{Bi}_{2} \mathrm{~S}_{3} \mathrm{NPs} \mathrm{Bi}^{(\text {III) }}$ cations are prone to hydrolysis to form $\mathrm{Bi}(\mathrm{OH})_{3}$ in an acidic environment. This study revealed that the BSA-mediated biomineralization of the BiNPs is an efficient and straightforward strategy, owing to the abundant amino acid residues on BSA that facilitate the binding of $\mathrm{Bi}^{3+}$ ions to BSA and improve their stability in an acidic environment. For targeted phototherapy, CD47-targeted PEGylated $\mathrm{Bi}_{2} \mathrm{Se}_{3}$ NPs were constructed following the protocol shown in Fig. 20b1. Anti-CD47 antibody (Ab) conjugated PEG- $\mathrm{Bi}_{2} \mathrm{Se}_{3}\left(\mathrm{Ab}-\mathrm{PEG}-\mathrm{Bi}_{2} \mathrm{Se}_{3}\right)$ NPs were shown to target tumor tissue and also increase the phagocytosis of cancer cells using macrophages to accelerate the effect of the PTT. CD47 is 
an overexpressed protein in most tumors that limits the macrophage phagocytosis effect by interacting with macrophage signal-regulated protein $\alpha(\operatorname{SIRP} \alpha){ }^{375}$ The developed NPs can block the crosstalking between CD47 and SIRP $\alpha$ as a "do not eat me" signal that inhibits the attack of macrophages, which accounts for $30-50 \%$ of the cells in the tumor mass. ${ }^{376}$ Selective accumulation of the $\mathrm{Ab}-\mathrm{PEG}-\mathrm{Bi}_{2} \mathrm{Se}_{3}$ NPs in a 4T1 mammary gland breast cancer tumor model, which was developed in female BALB/c mice, promoted the photothermal performance through stronger NIR absorbance, while enhancing macrophage-mediated phagocytosis of cancer cells via the inhibition of CD47-SIRP $\alpha$ affinity, as well as hyperthermia-induced release of stimulatory cytokines, such as IL-1 $\beta$ and tumor necrosis factor alpha (TNF- $\alpha$ ) from the tumor cells. This synergized the cancer suppression effect of the PTT (Fig. 20b2-b4) through the local stimulation of macrophages, NK cells and T lymphocytes against cancer cells via secreted inflammatory cytokines. ${ }^{377-380}$

In all of the above examples, a very high power laser was needed to heat the tumor to over $50{ }^{\circ} \mathrm{C}$ and achieve thorough ablation of the cancer cells. The induced hyperthermia to a tumor-killing temperature inevitably harms the surrounding healthy tissues. This drawback might limit the clinical application of PTT. Alternatively, mild hyperthermia of the cancer tissue at a relatively low level $\left(e . g ., 45{ }^{\circ} \mathrm{C}\right)$ is desirable; however, its killing effect is concerning and probably far from sufficient. Therefore, combining other therapeutic strategies for hyperthermia treatment under mild PTT have been proposed. ${ }^{381}$ For example, NIR-triggered nitric oxide (NO) releasing $\mathrm{Bi}_{2} \mathrm{~S}_{3}$ NPs were constructed for NO-sensitized mild PTT. $N, N^{\prime}$-Di-sec-butyl- $N, N^{\prime}$ dinitroso-1,4-phenylenediamine (BNN), which is a hydrophobic NO donor, was loaded on the surface of $\mathrm{Bi}_{2} \mathrm{~S}_{3}$ NPs (Fig. 21a and b). ${ }^{382}$ Since $\mathrm{BNN}$ is stable below $60{ }^{\circ} \mathrm{C}$, unexpected NO release under physiological conditions was avoided and just the high local temperature on the surface of $\mathrm{Bi}_{2} \mathrm{~S}_{3}$ NPs (induced by NIR laser) triggered the decomposition of BNN and NO release (Fig. 21c). Therefore, upon NIR irradiation at $808 \mathrm{~nm}$, mild PTT and on-demand NO release were simultaneously achieved to efficiently kill BEL-7402 hepatocellular carcinoma cells in vitro and also in vivo in female BEL-7402 tumor bearing mice (Fig. 21d and e). This strategy prohibits unwanted NO release in the systemic circulation and prevents its unexpected side effects. Instead, NO circumvents the heterogeneous heating distribution in the cancer tissue through the induced impairment of the autophagic self-repairing ability of cancer cells, which can subsequently accelerate the apoptotic shrinkage of the tumor tissue.

\subsection{Radiation monotherapy of cancer by $\mathrm{Bi}$ nanoplatforms}

RT is an important modality for cancer suppression that most often employs X-ray beams of intense energy to damage the genetic components of cancer cells. External beam therapy that uses a machine outside of the body to aim high-energy beams at a precise point on the body is the most common method used for RT. However, brachytherapy is another available approach in which radiation is targeted inside the body. Despite a plethora of recent attempts on RT, this method fails to fully eradicate tumors due to the (1) radiation insensitivity of some tumors due to their hypoxic nature, acidosis, and dense extracellular matrix, as well as (2) insufficient transfer of energy needed for ionization of the atoms that make up the DNA chain of the cells. ${ }^{52,209,383}$ In both scenarios, the production of free radicals in the cells is not high enough to destroy all cancer cells. Therefore, it is rather urgent to improve the efficiency of cancer radiotherapy via concentrating the radiation energy within the cancer tissues, while reversing their hypoxic microenvironment. ${ }^{384}$ Using heavy metal nano-radiosensitizers is a helpful approach to enhance the therapeutic efficiency of RT without increasing radiation doses to dangerously high levels that can non-selectively affect the normal function of surrounding tissues. ${ }^{385}$ BiNPs are used for this aim because of their strong photoelectric absorption and high generation of secondary electrons under radiation exposure. ${ }^{386-389}$ Huang et al. ${ }^{353}$ constructed a brachytherapy platform based on $\mathrm{Bi}_{2} \mathrm{~S}_{3}$ encapsulated PLGA nanocapsules ( $\left.\mathrm{Bi}_{2} \mathrm{~S}_{3} @ P L G A\right)$ using a water/oil/water $(\mathrm{W} / \mathrm{O} / \mathrm{W})$ emulsion approach. $\mathrm{Bi}_{2} \mathrm{~S}_{3}$ was used as a radiation sensitizer after exposure to gamma or X-ray irradiation and caused the death of PC3 prostate cells in vitro $\left(200 \mu \mathrm{g} \mathrm{mL}^{-1}\right)$ via the induction of DNA breakage. The PLGA capsules could be used as a CA for US, by which the radiation sources of brachytherapy could be guided into the desired position. In vivo findings in nude mice bearing a PC3 prostate carcinoma tumor xenograft demonstrated $c a .30 \%$ reduction in the tumor volumes by the BiNP mediated RT in comparison to the group that did not receive the NPs. Besides this, the immunohistochemical staining of the Ki67 protein of the tumor cells showed an obvious reduction in its expression in the NP treated group exposed to RT, while the other groups did not exhibit this. Nevertheless, the modulation of the abnormal tumor microenvironment through oxygenation was proposed as a useful strategy for further enhancement of the RT efficacy. ${ }^{369}$ For example, BSA-coated $\mathrm{Bi}_{2} \mathrm{~S}_{3}-\mathrm{MnO}_{2}$ nanocomposites were found to be capable of modulating hypoxic conditions in the tumor to enhance the efficacy of RT. ${ }^{390}$ After accumulation in the tumor, endogenous elevated $\mathrm{H}_{2} \mathrm{O}_{2}$ at the tumor site reacted with $\mathrm{MnO}_{2}$ to generate oxygen, leading to diminished hypoxia-associated resistance to RT.

In addition to the oxygen-deficient microenvironment of the tumor, which reduces the effect of RT, the abundance of antioxidants in the cancer tissue is another feature that may protect them against the damage from RT via the quenching of excess free radicals. ${ }^{391}$ For example, glutathione (GSH) is nearly $\sim 4$-fold higher in cancer cells compared to in normal cells ${ }^{392-394}$ and can decrease radiotherapeutic efficiency via its radical scavenging effect on ROS. ${ }^{395,396}$ In a pioneering and novel approach, Zhou et al. ${ }^{23}$ investigated the radiosensitizing effect of a nanomaterial composed of two high- $Z$ elements, i.e., $\mathrm{Bi}$ and $\mathrm{W}$ to achieve a better outcome for RT. They synthesized bismuth heteropolytungstate $\left(\mathrm{BiP}_{5} \mathrm{~W}_{30}\right)$ coupled with reduced graphene oxide (rGO) and surface modified with PVP (termed as PVP-PG) (Fig. 22a1). The hybrid composed of $\mathrm{BiP}_{5} \mathrm{~W}_{30}$ nanoclusters and rGO was prepared via an electrochemical reduction (ECR) process. The $\mathrm{BiP}_{5} \mathrm{~W}_{30}$ was found to be capable of depleting GSH by virtue of its unique electron structure and multi-electron properties meaning that it can catalyze the decomposition of $\mathrm{H}_{2} \mathrm{O}_{2}$ to ${ }^{\bullet} \mathrm{OH}$ to enhance ROS generation 

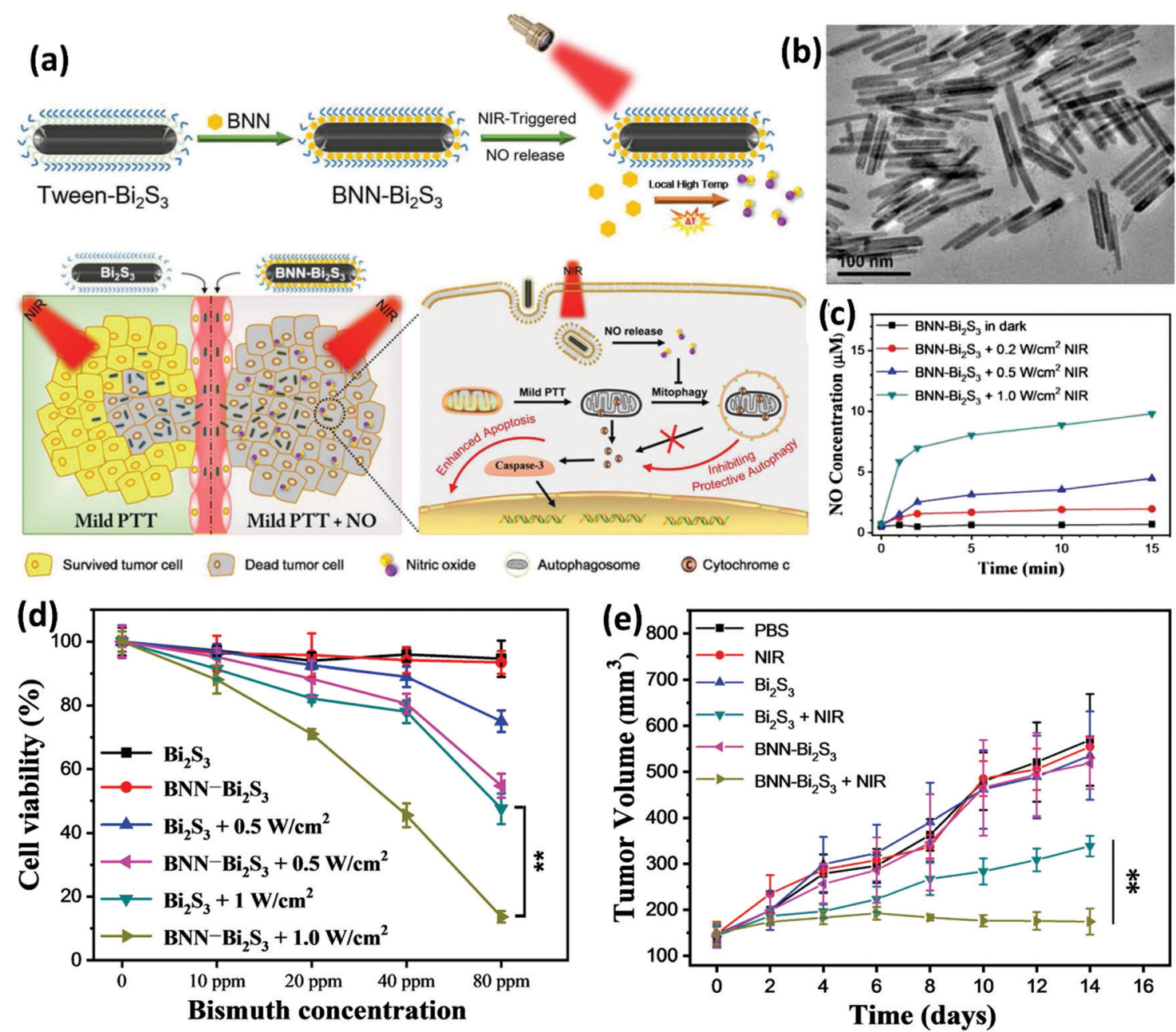

Fig. 21 (a) A schematic representation of the synthetic procedure and NIR-responsive NO release properties of $B N N-B_{2} \mathrm{~S}_{3}$. A synergistic mechanism involving mild $\mathrm{PTT}$ and $\mathrm{NO}$ in cancer therapy is shown to be a very favorable approach for avoiding damage to healthy tissues. (b) A TEM image of $\mathrm{Bi}_{2} \mathrm{~S}_{3} \mathrm{NRs}$. (c) The generation of $\mathrm{NO}$ from the $\mathrm{BNN}-\mathrm{Bi}_{2} \mathrm{~S}_{3}$ nanocomposite under $808 \mathrm{~nm}$ NIR laser irradiation with different power densities. (d) In vitro synergistic effects of mild $\mathrm{PTT}$ and $\mathrm{NO}$ achieved via the laser irradiation of the $\mathrm{BNN}-\mathrm{Bi}_{2} \mathrm{~S}_{3}$ nanocomposites. The cell viability of $\mathrm{BEL}-7402$ cells was decreased more for $\mathrm{BNN}-\mathrm{Bi}_{2} \mathrm{~S}_{3}$ treated samples compared to ones treated with $\mathrm{Bi}_{2} \mathrm{~S}_{3}$ at different concentrations under $808 \mathrm{~nm}$ laser irradiation. (e) The in vivo tumor volume of different groups of mice over fifteen days after various treatments. Adapted and reprinted with permission from ref. 382, Copyright 2019.

upon treatment with X-ray radiation. The rGO was found to further improve the radiocatalytic activity through enhanced electron-hole separation, while PVP rendered the NPs with exceptional stability in biofluids, with desirable biocompatibility. Although the photocurrents generated from both BiP5W30 and PVP-PG showed rapid response upon X-ray on/off switching, which is an indication of electron-hole separation, PVP-PG was able to generate a 4-fold higher photocurrent than that of the $\mathrm{BiP}_{5} \mathrm{~W}_{30}$ nanoclusters (Fig. 22a2). In fact, the presence of rGO more efficiently induced the separation of radiogenerated electron-hole pairs because of its two-dimensional planar structure that facilitates electron transportation. ${ }^{397}$ These radiogenerated electrons and holes were able to initiate the chemical reactions due to the robust photooxidative ability of the holes and photoreductive ability of the electrons, ${ }^{398}$ resulting in enhanced generation of hydroxyl radicals within a human cervix adenocarcinoma cell line (HeLa cells) upon X-ray irradiation (Fig. 22a3). In addition, the rGO was used for the absorption of NIR light and heat production as a mechanism confirmed to overcome the hypoxic microenvironment of the tumor and synergize the antitumor effects of RT, resulting in very effective suppression of tumor size in vivo (Fig. 22a4). A similar concept using two high- $Z$ elements in a single NP was also employed for the fabrication of $\mathrm{PVP}-\mathrm{Bi}_{2} \mathrm{WO}_{6}$ nanoplates that exhibited very high X-ray energy deposition performance, favorable photocurrent and photocatalytic-like radiocatalytic activity upon treatment with X-rays. This resulted in the effective separation of electron/hole $\left(\mathrm{e}^{-} / \mathrm{h}^{+}\right)$pairs and subsequently promoted the generation of cytotoxic ${ }^{\bullet} \mathrm{OH}$ (Fig. 22b1), confirmed by the detection of a $2^{\prime}, 7^{\prime}$-dichlorofluorescein (DCF) fluorescence signal in live cells using confocal laser scanning. It was obvious that the green fluorescence signals were highly emitted from HeLa cells treated with both $\mathrm{PVP}-\mathrm{Bi}_{2} \mathrm{WO}_{6}$ nanoplates and $\mathrm{X}$-rays, whereas only weak fluorescence signals could be detected in the cells treated with $\mathrm{PVP}-\mathrm{Bi}_{2} \mathrm{WO}_{6}$ nanoplates or X-rays alone. A clonogenic assay on human cervix adenocarcinoma cell line 
(a1)
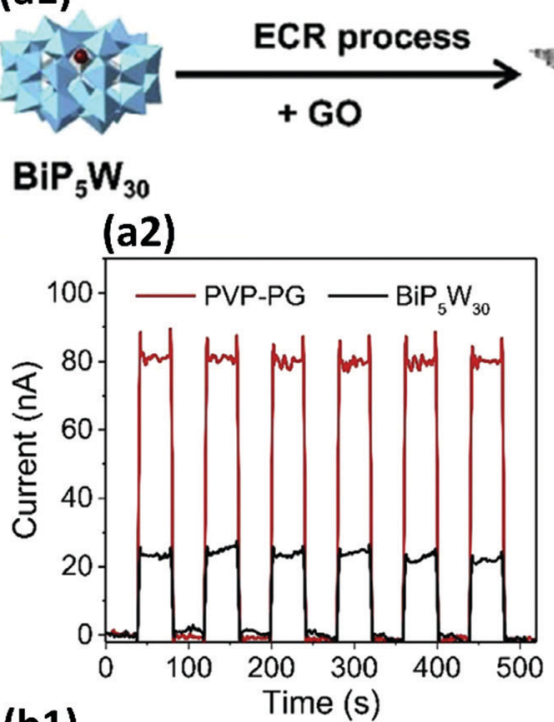

(b1)
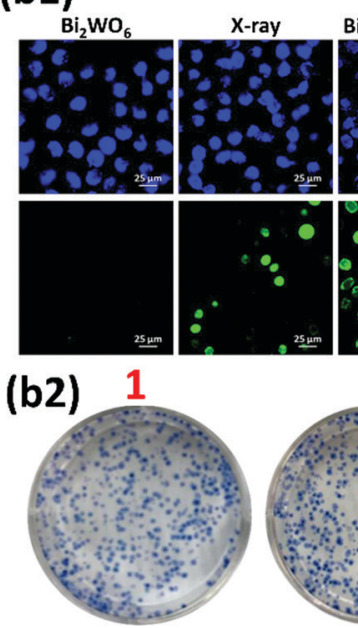

3

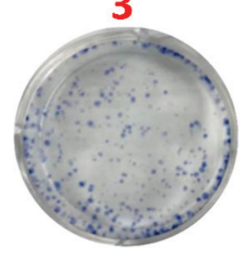

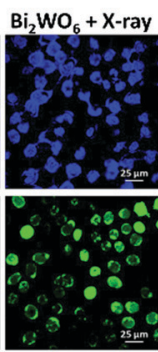

2

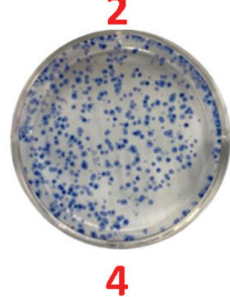

4

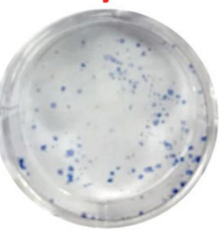

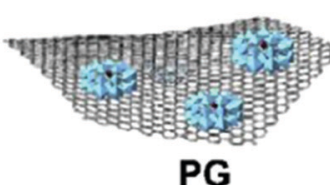

PG

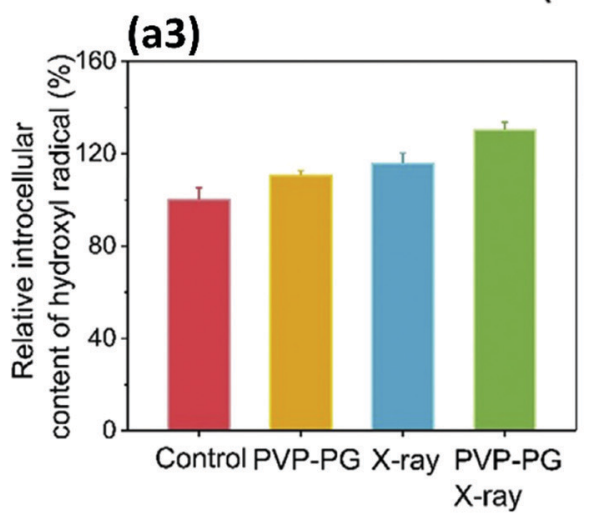

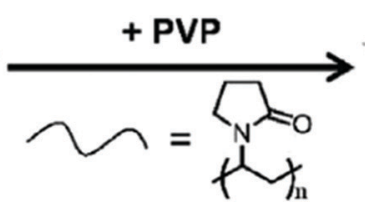

(a4) (c1)
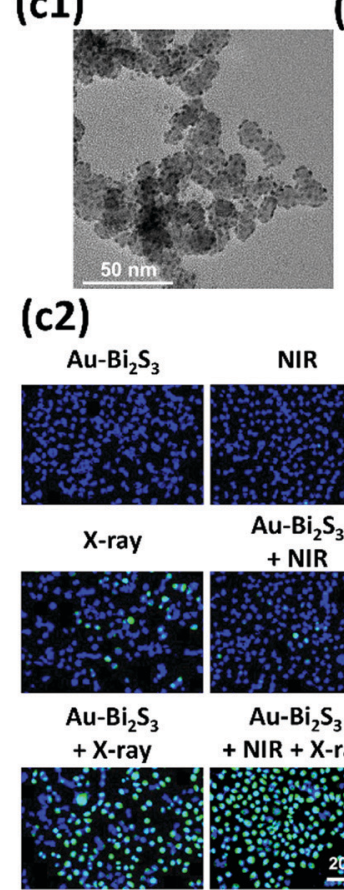

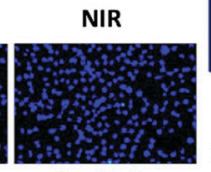

$\mathrm{Au}-\mathrm{Bi}_{2} \mathrm{~S}_{3}$

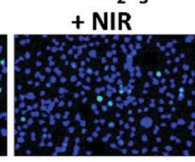

$\mathrm{Au}-\mathrm{Bi}_{2} \mathrm{~S}_{3}$ + NIR + X-ray
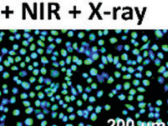

(c3)

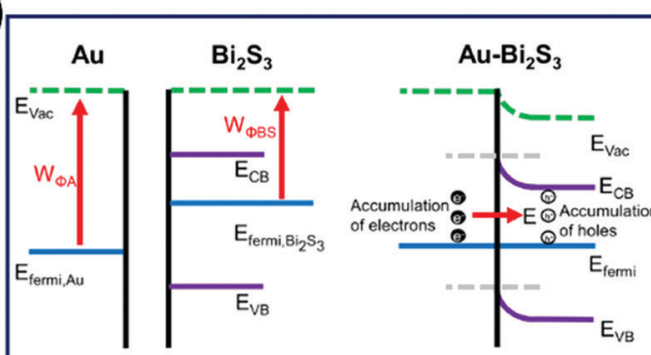

Before contac

After contact

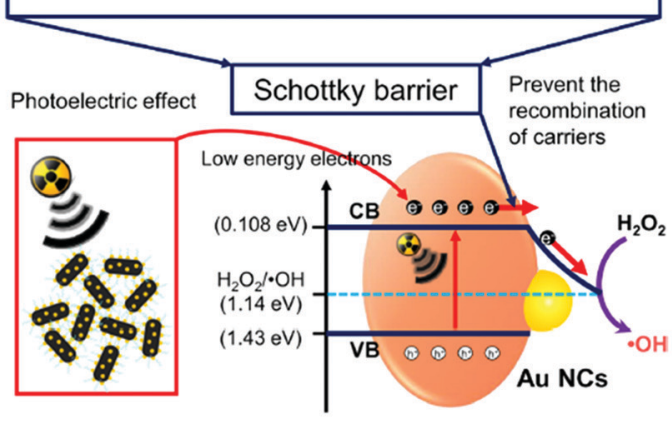

Potential (SHE)

$\mathrm{Bi}_{2} \mathrm{~S}_{3} \mathrm{NRS}$

Fig. 22 (a1) The synthesis procedure of PVP-PG. (a2) X-ray induced photocurrent with $\mathrm{BiP}_{5} \mathrm{~W}_{30}$ clusters and PVP-PG through the on/off switching of $X$-ray irradiation. (a3) The relative content of hydroxyl radicals in HeLa cells after different treatments. (a4) Digital images of the dissected tumors of each group. Adapted and reprinted with permission from ref. 23, Copyright 2019. (b1) Fluorescence images of ROS generation in HeLa cells treated differently and stained using Hoechst 33342 and DCFH-DA. (b2) Digital photographs of the colony formation of HeLa cells (1) without any treatment, and after treatment with (2) $100 \mu \mathrm{g} \mathrm{mL}{ }^{-1} \mathrm{PVP}-\mathrm{Bi}_{2} \mathrm{WO}_{6}$ nanoplates, (3) X-rays and (4) PVP-Bi $\mathrm{WO}_{6}+\mathrm{X}$-ray irradiation (50 kV, $\left.75 \mu \mathrm{A}, 30 \mathrm{~s}\right)$. Adapted and reprinted with permission from ref. 402, Copyright 2019. (c1) A TEM image of the Au-Bi $\mathrm{S}_{3}$ nanocomposite. (c2) Fluorescence images of HeLa cells after different treatments and staining with Hoechst 33342 (blue) and DCF (green) for the imaging of nuclei and free radical generation, respectively. (c3) A schematic demonstration of the plausible mechanism involved in enhanced free radical generation via an X-ray-induced catalytic progress by Schottky-type $\mathrm{Au}-\mathrm{Bi}_{2} \mathrm{~S}_{3}$ heterostructures When $\mathrm{Bi}_{2} \mathrm{~S}_{3}$ and $\mathrm{Au}$ come into contact, electrons from $\mathrm{Bi}_{2} \mathrm{~S}_{3}$ transfer to $\mathrm{Au}$ at the interface until equilibrium is reached between $E_{\mathrm{Fermi}}, \mathrm{B}_{2} \mathrm{~S}_{3}$ and $E_{\mathrm{Fermi}} \mathrm{Au}$, resulting in new $E_{\mathrm{Fermi}}$ generation. This process leads to the formation of a positive charge layer at the surface of $\mathrm{Bi}_{2} \mathrm{~S}_{3}$ and the accumulation of negative electrons on the surface of $\mathrm{Au}$, leading to the deformation of the band structure between $\mathrm{Au}_{\mathrm{u}}$ and $\mathrm{Bi}_{2} \mathrm{~S}_{3}$, where the surface potential barrier, known as the Schottky barrier is formed. Adapted and reprinted with permission from ref. 403, Copyright 2019.

(HeLa cells) confirmed the radiosensitizing effect of the PVP$\mathrm{Bi}_{2} \mathrm{WO}_{6}$ nanoplates via a high amount of ROS generation. The cells were treated with the NPs $\left(100 \mu \mathrm{g} \mathrm{mL}^{-1}\right)$ for $6 \mathrm{~h}$ before washing and exposure to X-rays $(50 \mathrm{kV}, 75 \mu \mathrm{A})$ for $30 \mathrm{~s}$ before cell culturing and staining with Giemsa dye to visually count the number of cell colonies. As can be observed in Fig. 22b2, 
the control and $\mathrm{Bi}_{2} \mathrm{WO}_{6}$ nanoplate-treated groups (1 and 2) produced more colonies than those of the X-ray (3) or $\mathrm{Bi}_{2} \mathrm{WO}_{6}+$ $\mathrm{X}$-ray (4) treated groups. According to all of the above examples, development of oxygen-independent RT approaches that overcome the hypoxia of the tumor tissue is currently the key for further development of novel multifunctional radiosensitizers. PVP-modified Schottky-type heterostructured $\mathrm{Au}-\mathrm{Bi}_{2} \mathrm{~S}_{3}$ NPs (Fig. 22c1) are another example material that can deposit a high radiation dose within tumors in the form of high energy electrons and efficiently eradicate cancer without suffering from the oxygen-dependent radio-sensitizing behavior of the NPs in hypoxic tumors. The generation of free radicals in HeLa cells was evaluated by employing $2^{\prime}, 7^{\prime}$-dichlorofluorescin diacetate (DCFH-DA) as a probe for the detection of free radicals. The green fluorescence of DCF was then detected, showing no signal in the $\mathrm{Au}-\mathrm{Bi}_{2} \mathrm{~S}_{3}$ and NIR treated groups, a very weak signal in the $\mathrm{X}$-ray and $\mathrm{Au}-\mathrm{Bi}_{2} \mathrm{~S}_{3}+\mathrm{NIR}$ groups, and a very strong signal in the $\mathrm{Au}-\mathrm{Bi}_{2} \mathrm{~S}_{3}+\mathrm{X}$-ray and $\mathrm{Au}-\mathrm{Bi}_{2} \mathrm{~S}_{3}+\mathrm{NIR}+\mathrm{X}$-ray groups with the latter showing the biggest effect on ROS generation (Fig. 22c2). This feature of Schottky-type heterostructured $\mathrm{Au}-\mathrm{Bi}_{2} \mathrm{~S}_{3}$ is associated with the generation of a Schottky barrier between the metal, $\mathrm{Au}$, and semiconductor, $\mathrm{Bi}_{2} \mathrm{~S}_{3}$, which can trap the electrons generated by the X-ray treatment and transfer them to the $\mathrm{Au}$, resulting in the prevention of the recombination of the electron-hole pairs (Fig. 22c3). ${ }^{399-401}$ Thereafter, due to the matched potential between the conduction band of the $\mathrm{Bi}_{2} \mathrm{~S}_{3}$ NPs and overexpressed $\mathrm{H}_{2} \mathrm{O}_{2}$ within the tumor, the $\mathrm{Au}-\mathrm{Bi}_{2} \mathrm{~S}_{3}$ nanocomposite induces the decomposition of intracellular $\mathrm{H}_{2} \mathrm{O}_{2}$ into highly toxic ${ }^{\bullet} \mathrm{OH}$ for selective killing of HeLa cells.

\subsection{Combined cancer therapy and theranostics based on BiNPs}

Despite the great efforts devoted to cancer monotherapy over the past decades, efficient eradication of tumors at an early stage remains a big challenge. Most tumors are not detected until they become large in size, increasing the risk of poor therapeutic outcome and metastasis. An alternative approach is combining more than one therapeutic method with diagnostic tools to overcome the shortcomings of the individual therapies. ${ }^{404,405}$ The simultaneous use of imaging modalities and multi-therapy requires versatile nanotheranostic agents. ${ }^{209}$ In this context, BiNPs were proposed, owing to their diverse applications, such as CT imaging, PAI, PTT, and RT. ${ }^{406}$ For example, ultrasmall BSA stabilized $\mathrm{Bi}_{2} \mathrm{Se}_{3}$ nanodots have exhibited a high photothermal conversion efficiency $(\eta=50.7 \%)$ for PTT, while serving as PAI and CT multimodal imaging-guided synergetic sensitizers for radiotherapy. ${ }^{407}$ The average size of the NPs was $2.7 \pm 0.3 \mathrm{~nm}$ and they were simply synthesized through the reaction of bismuth chloride with hydroxyethylthioselenide in aqueous solution under ambient conditions. Most studies have reported the synthesis of BiNPs in hydrophobic organic solvents to avoid the fast hydrolysis of $\mathrm{Bi}^{3+}$. However, these NPs are large in size and post synthetic surface modification was needed to make them water soluble. Their large size might also lead to long metabolism and induce more side effects. ${ }^{252,408}$ Therefore, in the abovementioned study, the advantage of preparing hydrophilic and biocompatible $\mathrm{Bi}_{2} \mathrm{Se}_{3}$ BiNPs by dissolving water soluble bismuth salts in an acidic solution is highly significant as it could prevent the hydrolysis of $\mathrm{Bi}^{3+}$ ions, leading to the production of ultrasmall NPs.

The intrinsic PTT, RT, CT, and PAI potentials of BiNPs have also been combined with other imaging modalities or therapeutic strategies, such as MRI, chemotherapy and immunotherapy, to develop novel theranostics. For example, monodisperse $\mathrm{Gd}_{2} \mathrm{O}_{3} / \mathrm{Bi}_{2} \mathrm{~S}_{3}$ hybrid nanodots (Gd/Bi-NDs) were prepared for simultaneous CT, PAI, MRI and PTT. ${ }^{409} \mathrm{Bi}\left(\mathrm{NO}_{3}\right)_{3}$ and $\mathrm{GdCl}_{3}$ were mixed with BSA in water under vigorous agitation before adjusting the $\mathrm{pH}$ to 12 . Under basic conditions, the abundant active groups of the albumin molecules were found to efficiently bind to the metal ions of $\mathrm{Bi}^{3+}$ and $\mathrm{Gd}^{3+}$ to generate crystal nuclei. Numerous cysteine residues were also released to act as a precursor for the nanoprecipitation of $\mathrm{Bi}_{2} \mathrm{~S}_{3}$ nanocrystals. Nanoprecipitation of the $\mathrm{Gd}^{3+}$ ions also occurred under basic conditions as a result of their binding to albumin molecules and subsequent formation of $\mathrm{Gd}_{2} \mathrm{O}_{3}$ nanocrystals through the reaction of hydroxyl groups with $\mathrm{Gd}^{3+}$ ions (Fig. 23a1). In addition to NIR fluorescence imaging that showed the in vivo retention behavior of the Cypate-labeled NPs in epithelial 4T1 mammary gland tumors for nearly $48 \mathrm{~h}$ (Fig. 23a2), PAI, MRI, and CT imaging were monitored with maximum PA and MR intensity at $12 \mathrm{~h}$ and efficient CT contrast during $2 \mathrm{~h}$ postinjection (Fig. 23a3-a5). The relaxivity of Gd/Bi-NDs was found to be around $26.8 \times 10^{-3} \mathrm{~m}^{-1} \mathrm{~s}^{-1}$, which is eight-fold higher than that of gadolinium-diethylenetriamine pentaacetic acid (Gd-DTPA, $3.2 \times 10^{-3} \mathrm{~m}^{-1} \mathrm{~s}^{-1}$ ). The CT contrast of these NP is also $c a$. 2.5-fold higher than that of Iopromide. Efficient cellular uptake, high tumor accumulation, favorable in vivo clearance, negligible acute toxicity, and effective photothermal tumor ablation without recurrence makes these monodisperse multicomponent nanoagents highly promising as efficient cancer theranostic agents.

Combined phototherapy and immunotherapy using BiNPs is another novel approach recently highlighted for multi-therapeutic cancer ablation. Song et al. ${ }^{410}$ prepared immune-adjuvant encapsulated $\mathrm{Bi}_{2} \mathrm{Se}_{3}$ nanocages for photothermal-improved PD-L1 checkpoint blockade metastasis therapy. As shown in Fig. 23b, MnSe nanocubes were used as a template to fabricate $\mathrm{Bi}_{2} \mathrm{Se}_{3}$ nanocages (NCs). First, manganese acetate tetrahydrate was added into a mixture of 1-octadecene, oleylamine and oleic acid at room temperature, followed by heating at $120{ }^{\circ} \mathrm{C}$ for 60 min to obtain solution no. 1. Next, selenium was dispersed in a solution of oleylamine and 1-dodecanethiol, followed by its addition into solution no. 1 and was held at $220{ }^{\circ} \mathrm{C}$ for $2 \mathrm{~h}$. After cooling to $50{ }^{\circ} \mathrm{C}$, a solution of 1-octadecene containing bismuth neodecanoate was added under a constant flow of nitrogen and the mixture was heated to $180{ }^{\circ} \mathrm{C}$ for $0.5 \mathrm{~h}$ under vigorous stirring. The solution was then cooled down to room temperature and oleylamine coated $\mathrm{Bi}_{2} \mathrm{Se}_{3}$ NCs were collected by centrifugation for further decoration with PEG (termed as NC-PEG). Imiquimod (R848), a toll-like receptor-7 agonist, was loaded into the NCs to generate strong anti-cancer immune responses by NC-PEG/R848. NIR mediated PPT using this immunogenic NP not only ablated the light-exposed cancer 

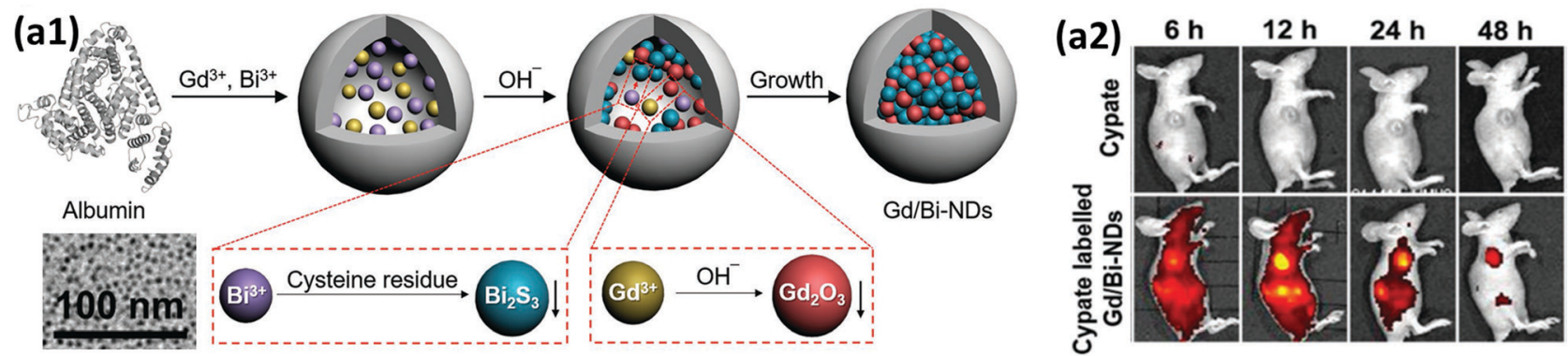

(a3)
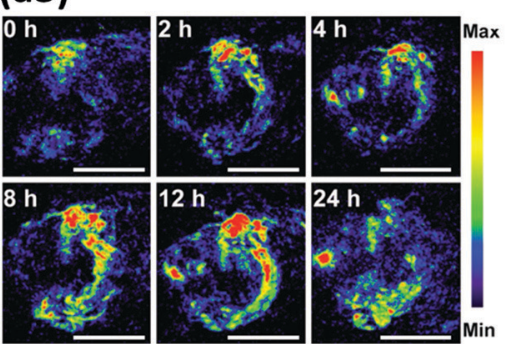

(a4)
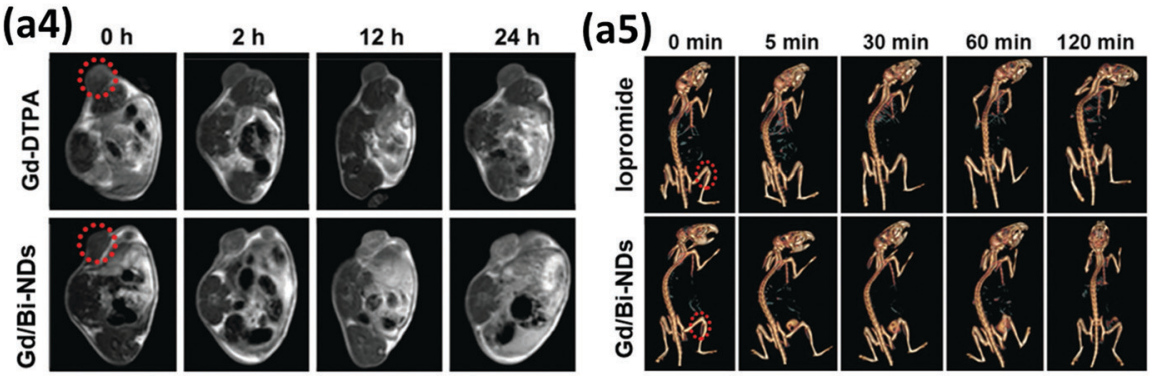

(b)
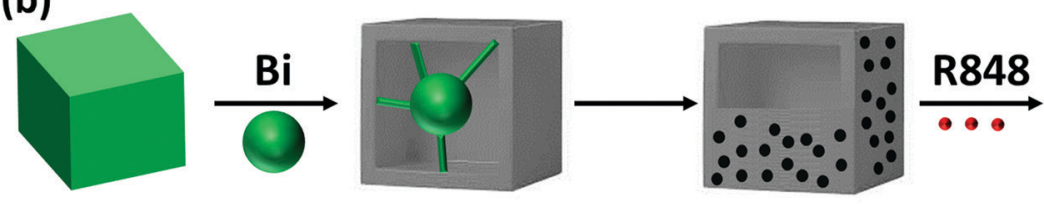

$\mathrm{Bi}_{2} \mathrm{Se}_{3} \mathrm{NC}$

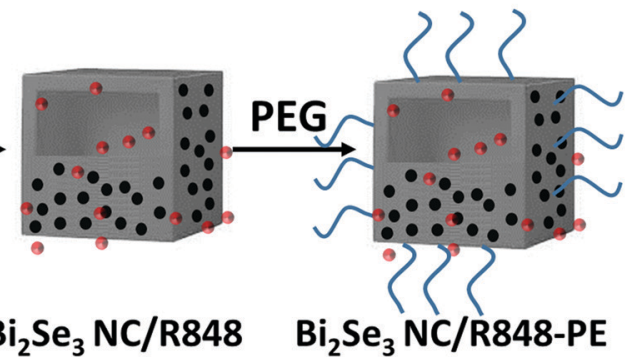

Fig. 23 (a1) A schematic illustration of Gd/Bi-NDs nanodots synthesized through separate nanoprecipitation reactions in a single albumin solution and a corresponding TEM image of the NPs. (a2) In vivo NIR fluorescence imaging of mice injected with Cypate-labeled Gd/Bi-NDs and free Cypate at a dose of $20 \mu \mathrm{mol} \mathrm{kg}{ }^{-1} \mathrm{Bi}$. (a3) In vivo PAl of xenografted tumors from mice injected with Gd/Bi-NDs at a dose of $20 \mu \mathrm{mol} \mathrm{kg}{ }^{-1} \mathrm{Bi}$ over $24 \mathrm{~h}$ post-injection. (a4) In vivo MRI of mice injected with Gd/Bi-NDs and Gd-DTPA at a dose of $20 \mu \mathrm{mol} \mathrm{kg}{ }^{-1} \mathrm{Gd}$ over 24 h post-injection. (a5) In vivo CT imaging of mice i.t. injected with Gd/Bi-NDs and lopromide at a dose of $125 \mu \mathrm{mol} \mathrm{kg}{ }^{-1} \mathrm{Bi}$ over $2 \mathrm{~h}$. Adapted and reprinted with permission from ref. 409 , Copyright 2018. (b) A schematic illustration of the synthesis procedure of $\mathrm{Bi}_{2} \mathrm{Se}_{3} \mathrm{NC}-\mathrm{PEG} / \mathrm{R} 848$. Reproduced from ref. 410, Copyright 2019.

tissue, but also induced strong anti-cancer immunity to suppress the growth of a distant secondary tumor through the increased secretion of inflammatory cytokines, including TNF$\alpha$, interferon $\gamma$ (IFN- $\gamma$ ), and IL-12p40. Co-Administration of this formulation with an anti-PD-L1 antibody further silenced the immune resistance based on a PD-L1 checkpoint blockade to suppress the primary tumor, considered as a synergistic approach for the inhibition of tumor metastasis. In addition to these examples, there are other studies on combined therapy with BiNPs, which are summarized in Table 3, indicating the potential of these nanomedicines for future investigation and creation of versatile multifunctional theranostics.

\section{Bi-Based biosensors}

Biosensors are analytical devices that translate amounts of biochemical substances into a measurable signal, which is directly related to the level of the analyte. The main components of biosensors include a bioreceptor that identifies the analyte and generates a signal, a transducer that converts signals into quantifiable and user-friendly electronic pulses, and a reader device to represent the signal in a displayable format. ${ }^{425}$ Electrochemical and photoelectrochemical (PEC) technologies (converting photoirradiation to electrical signals (current or potential) ${ }^{426,427}$ are the main detection methods used in the development of biosensing devices for drug discovery, food safety, environmental monitoring, and more importantly medical diagnostics through the detection of various analytes within complex body fluids (e.g. saliva, blood, urine). ${ }^{425,428}$ Among the various types of biosensors, nanobased ones exhibit great promise for meeting the demands of clinical diagnosis due to their attractive features of a high surface area, high stability and sensitivity, high selectivity, simplicity of construction, reproducibility, speed of response and low cost of analysis. ${ }^{425}$ Different types of nanomaterials, such as carbon-based nanostructures, metal NPs, magnetic NPs, and polymeric NPs have been utilized as both bioreceptors and transducers to improve the performance of electrochemical, optical, mass-based, or calorimetric sensors due to the desirable strength of the signal detection. ${ }^{429,430}$ Various types of Bi-based nanomaterials, such as its metallic forms, binary 


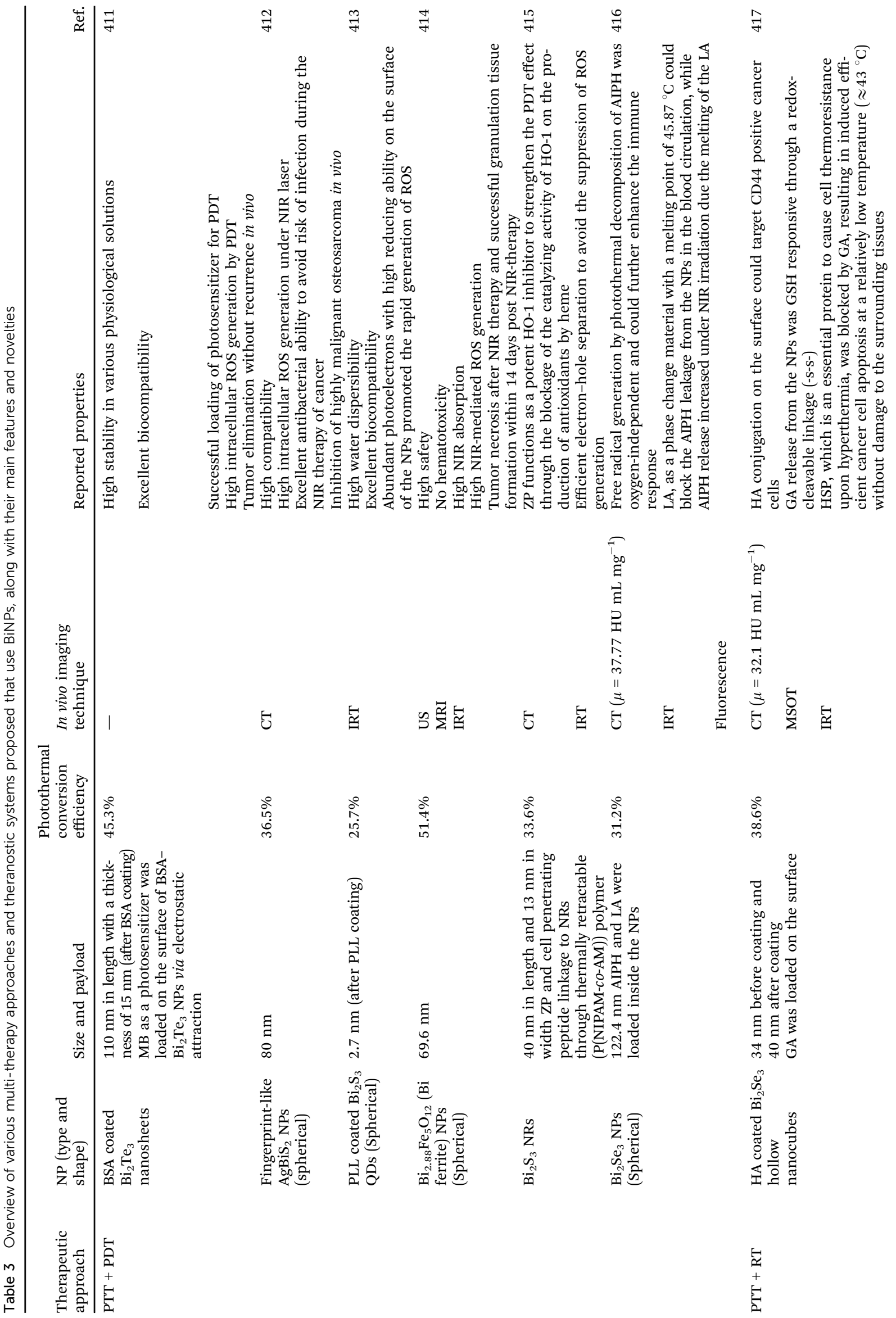


Review Article

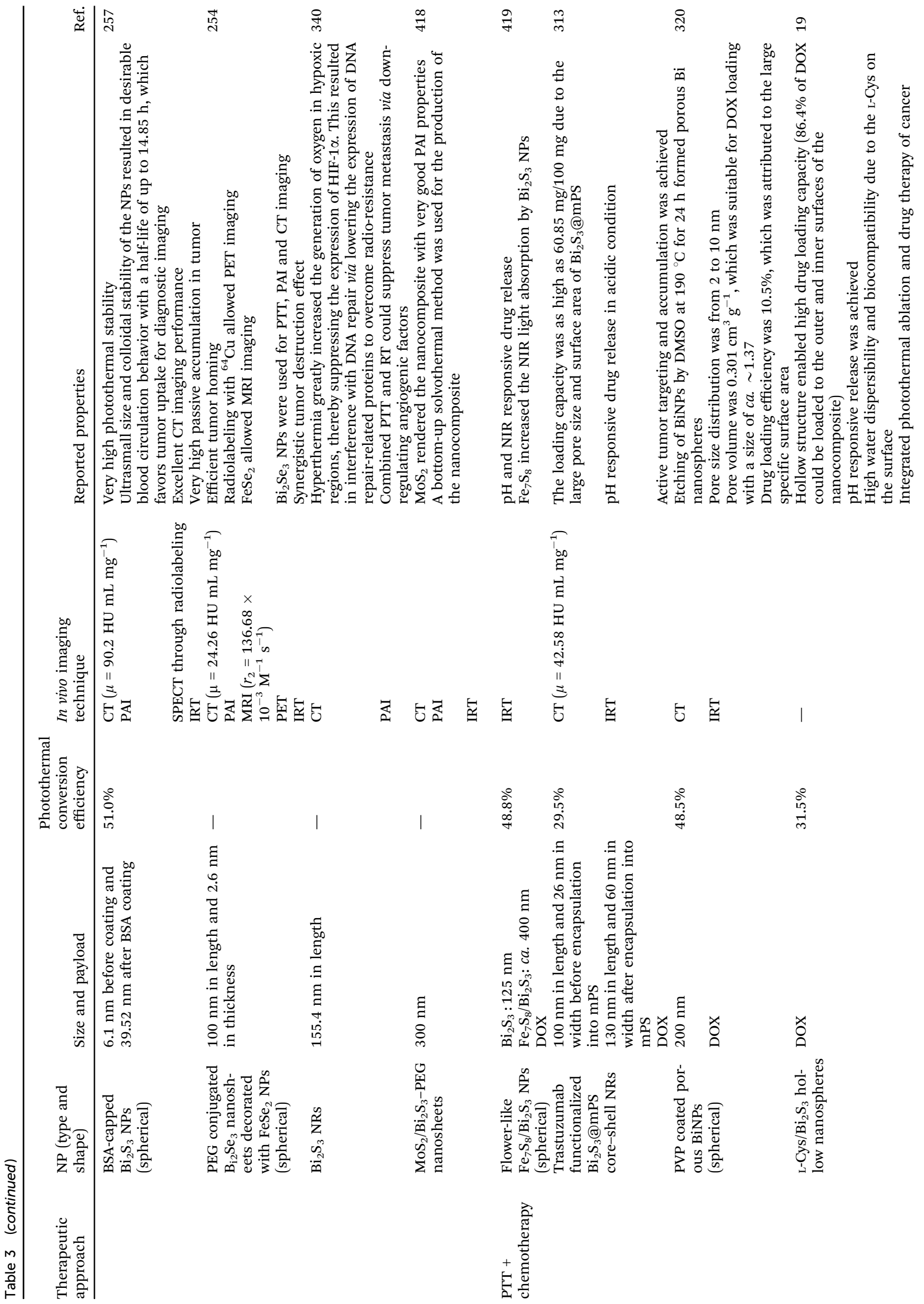




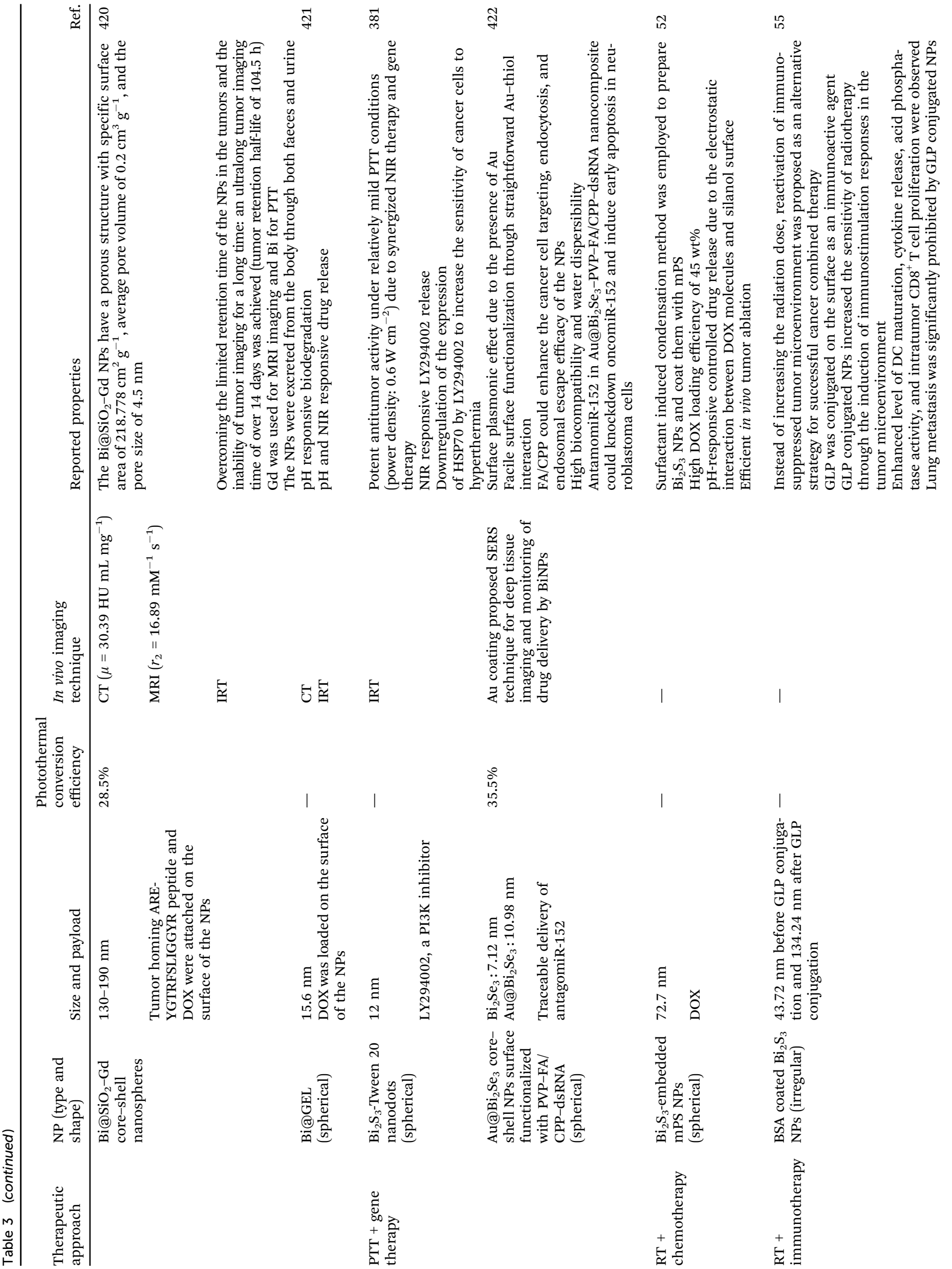




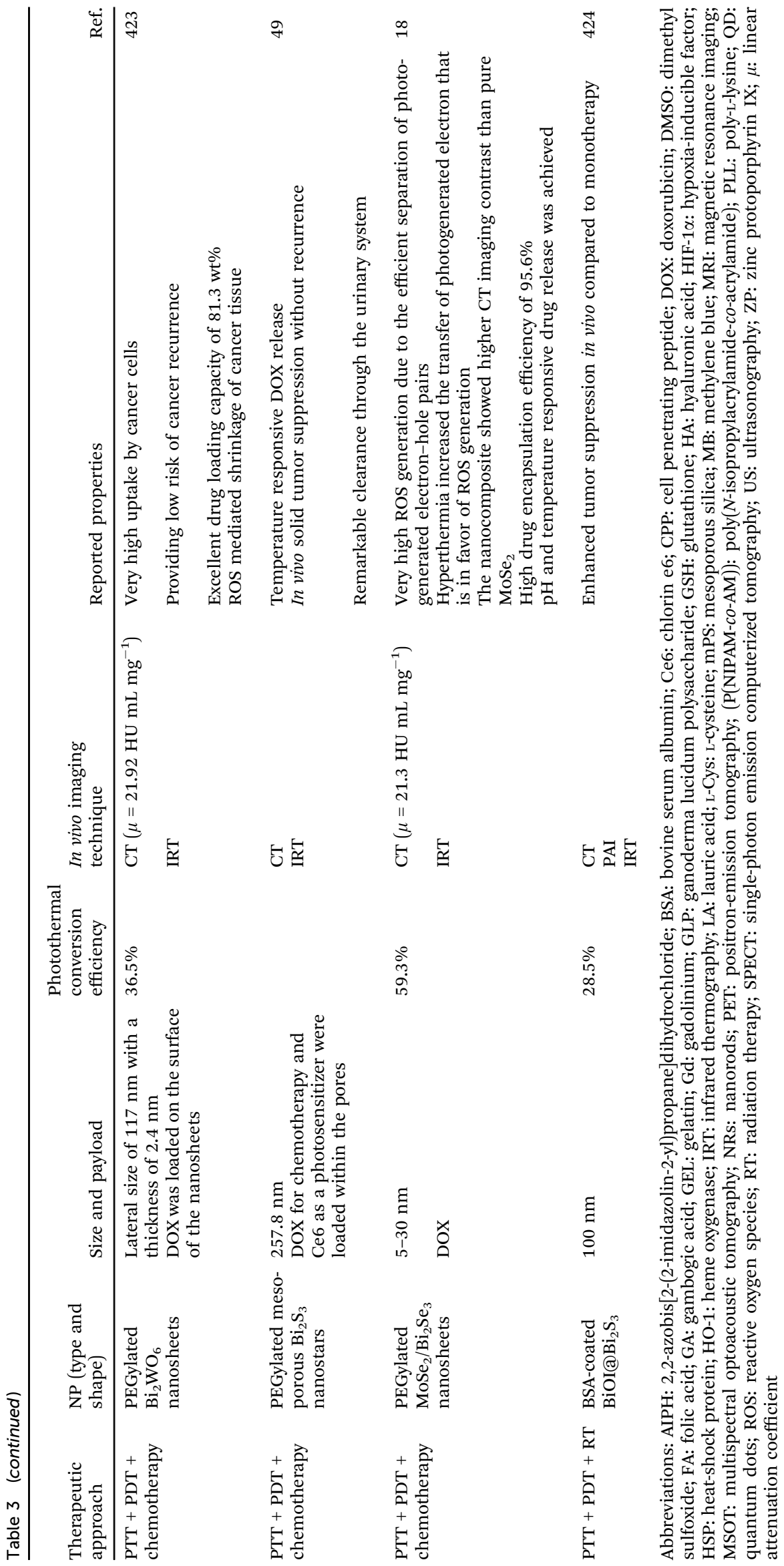


oxides $\left(\mathrm{Bi}_{2} \mathrm{O}_{3}\right)$ and multicomponent oxides (e.g., $\mathrm{BiVO}_{4}$ ), selenides $\left(\mathrm{Bi}_{2} \mathrm{Se}_{3}\right)$, sulfides $\left(\mathrm{Bi}_{2} \mathrm{~S}_{3}\right)$ and oxyhalides (BiOX) have newly emerged for use in biosensing applications. ${ }^{427}$ The unusual performance of BiNPs as a biosensor relies on their unique physicochemical properties that include high electrical resistance and low thermal conductivity, high surface-tovolume ratio, high surface free energy, excellent visible-light activity, and favorable properties for biomolecule adsorption. ${ }^{431,432}$ In addition, Bi materials are non-toxic, biocompatible, chemically inert, highly stable, environmentally friendly, and provide high resolution of the voltammetric responses and desirable limit of detection (LOD), while their synthesis is usually easy and affordable. ${ }^{431,432}$ Due to the above-described properties, Bi is considered as an alternative to toxic sensing electrodes, such as metallic mercury, since various forms of its composites can be simply prepared, mainly for electrochemical and PEC biosensing using different metals. ${ }^{427,433-435}$ Electrodes incorporating Bi in the bulk state, ${ }^{436,437}$ BiNPs of various shapes and sizes, ${ }^{433,438-444}$ and Bi film electrodes (BiFEs) ${ }^{445-448}$ have been developed for the determination of various analytes with high sensitivity and low background signal and their general applications have previously been well-reviewed. ${ }^{427,431,449,450}$ Herein, our focus will be on the biomedical applications of sensors developed using different chemical forms and shapes of BiNPs and BiFEs.

\subsection{Bismuth oxides $\left(\mathrm{BiO}_{x}\right)$ for biosensing}

$\mathrm{BiO}_{x}$ materials possess interesting physical properties for biosensing technology, recognized as p-type electronic semiconductors with a large energy band-gap of around $2.1 \mathrm{eV}$, a high refractive index, favorable dielectric permittivity, photoconductivity and photoluminescence properties. ${ }^{451-453}$ As a result of their chemical inertness and in vivo biocompatibility, $\mathrm{BiO}_{x}$ materials have recently been proposed for gas sensing, as a coating for photovoltaic cells, as well as in biosensor fabrication. ${ }^{45,455}$ Nanosized $\mathrm{BiO}_{x}\left(\right.$ nano- $\left.\mathrm{BiO}_{x}\right)$ shows the prominent features of a high surface area, high surface free energy, and electrochemical stability, which plays meaningful roles in analyte adsorption and detection at low concentrations. These physical properties have resulted in the development of $\mathrm{BiO}_{x}$ materials for use in glucose sensing, ${ }^{433}$ DNA hybridization, ${ }^{442}$ and $\mathrm{H}_{2} \mathrm{O}_{2}$ detection. ${ }^{441}$ For example, Ding et al. ${ }^{433}$ developed a nanostructured amperometric glucose biosensor based on spreading a solution mixture of glucose oxidase (GOD) and polycrystalline nano- $\mathrm{BiO}_{x}$ (composed mainly of $\mathrm{BiO}_{x}$ nanorods and nanosheets) onto a platinum disk electrode, followed by drying and 15 min of treatment using saturated glutaraldehyde vapor in order to induce the chemical cross-linking of the entrapped enzyme molecules on the surface of the electrode. Then, the amperometric measurement of glucose levels was achieved by the GOD mediated oxidation of $\beta$-D-glucose to gluconic acid and hydrogen peroxide in the presence of $\mathrm{O}_{2}$. The GOD/nano-BiOx electrode was finally able to oxidize the enzymatically generated hydrogen peroxide at $0.5 \mathrm{~V}$. Linear measurement of glucose levels was observed over a concentration range of $1 \times 10^{-6}$ to $1.5 \times 10^{-3} \mathrm{M}$ with a detection limit of $4 \times 10^{-7} \mathrm{M}$ and a sensitivity of $51.0 \pm 0.4 \mathrm{~mA} \mathrm{M}^{-1} \mathrm{~cm}^{-2}$.
This achievement is associated with the photovoltaic effect of the NPs, which assists the enzymatic reaction through the catalysis of oxidation/reduction reactions. This biosensor exhibited favorable thermodynamic stability and life span due to the protective effect of the $\mathrm{BiO}_{x}$ matrix on the degradation of GOD enzyme. This can be explained by partial enzyme embedding or encapsulation in nano- $\mathrm{BiO}_{x}$ during the procedure of enzyme immobilization, resulting in improved thermal resistance, which might also explain the extended storage stability of over 2 months.

To enhance the capacity of converting photoirradiation into an electrical signal in PEC biosensors and overcome the high toxicity, easy photo-bleaching, and the narrow optical response range of the classical semiconductor group (II-VI) nanohybrids, ${ }^{427,456}$ multicomponent $\mathrm{BiO}_{x}$ and $\mathrm{BiOX}(\mathrm{X}=\mathrm{F}, \mathrm{Cl}$, $\mathrm{Br}$, and I), such as bismuth oxybromide (BiOBr) ${ }^{457}$ bismuth iodide oxide (BiOI), ${ }^{202} \mathrm{Bi}_{2} \mathrm{O}_{3},{ }^{458} \mathrm{Bi}$ vanadate $\left(\mathrm{BiVO}_{4}\right),{ }^{201,459} \mathrm{BiFeO}_{3},{ }^{460}$ and perovskites members like $\mathrm{Bi}_{2} \mathrm{WO}_{6}{ }^{461,462}$ have drawn significant attention, particularly in the design of PEC immunosensors. They possess superb properties under visible light irradiation, such as high chemical stability, low toxicity, a large energy band gap, high refractive index, dielectric permittivity, high oxygen-ion conductivity, and pronounced photoconductivity and photoluminescence properties. ${ }^{448,452} \mathrm{Zhu}$ et al. ${ }^{462}$ fabricated $\mathrm{Bi}_{2} \mathrm{WO}_{6}$ nanosheets to be incorporated into an organicinorganic heterojunction (interface that occurs between two layers or regions of dissimilar crystalline semiconductors) of a polypyrrole@ $\mathrm{Bi}_{2} \mathrm{WO}_{6}$-based electrode, yielding a sensitive PEC immunoassay for the early detection of creatine kinase-MB, present a few hours after a heart attack. Fan et al. ${ }^{457}$ developed an ultrasensitive label-free immunosensor based on a bismuth oxybromide/silver sulfide $\left(\mathrm{BiOBr} / \mathrm{Ag}_{2} \mathrm{~S}\right)$ composite with high visible-light PEC activity for the detection of insulin (Fig. 24a). An indium tin oxide (ITO) electrode was used as a platform for the immobilization of $\mathrm{BiOBr}$ on its surface. Thioglycolic acid (TGA) was then used for the modification of the BiOBr modified ITO electrode through dropping of its aqueous solution on the surface and drying under an infrared lamp. Next, $\mathrm{Ag}_{2} \mathrm{~S}$ was grown in situ on the surface of the ITO/BiOBr/TGA electrode via the addition of a solution mixture of the $\mathrm{AgNO}_{3}$ and $\mathrm{Na}_{2} \mathrm{~S}$. After washing, dopamine (DA) was added to the electrode to form a PD film. PD has very high adhesivity to biomolecules, enabling efficient attachment of the anti-insulin antibody onto the surface of the ITO/BiOBr/TGA $/ \mathrm{Ag}_{2} \mathrm{~S} / \mathrm{PD}$ electrode. BSA was finally dropped onto the electrode at room temperature to block the nonspecific binding sites and make the device ready for insulin detection. In this study, a high and stable photocurrent signal was obtained by adding the excellent antioxidant ascorbic acid (AA) to the sample being tested. Holes generated by the illuminated $\mathrm{Ag}_{2} \mathrm{~S}$ NPs oxidize AA, facilitating the separation of photogenerated holes and electrons $\left(\mathrm{h}^{+} / \mathrm{e}^{-}\right)$to achieve perfect photoelectrochemical activity and photocurrent response. ${ }^{463}$ The separation of photogenerated $\mathrm{h}^{+} / \mathrm{e}^{-}$is inefficient under visible light due to the wide band-gap of $\mathrm{BiOBr}$, and therefore, $\mathrm{Ag}_{2} \mathrm{~S}$ NPs are used to promote electron transition under visible light (Fig. 24b). The presence of PD also enhances the visible light 

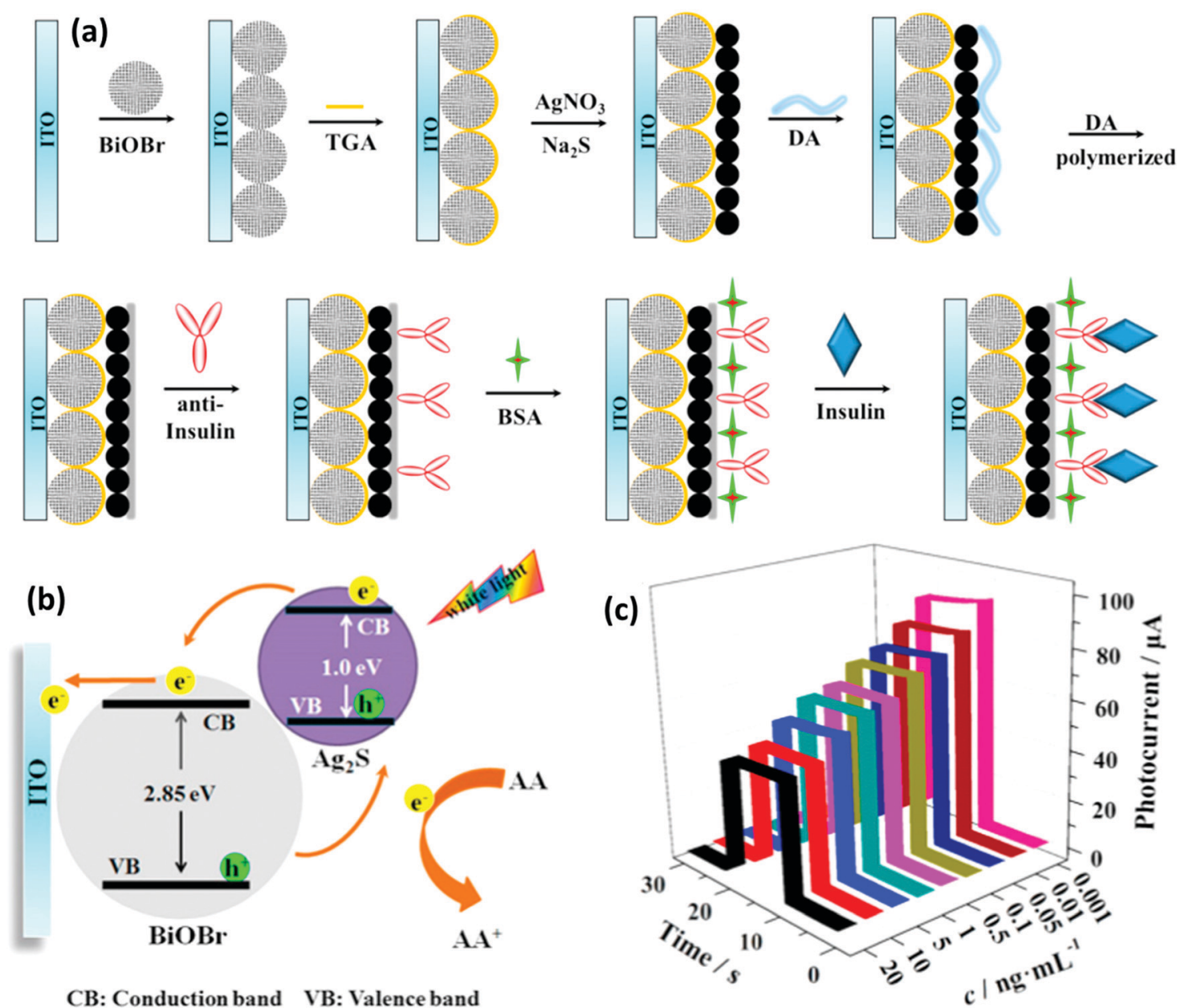

CB: Conduction band VB: Valence band

Fig. 24 (a) A schematic demonstration of the fabrication process of a $\mathrm{BiOBr} / \mathrm{Ag}_{2} \mathrm{~S} \mathrm{PEC}$ immunosensor. $\mathrm{Ag}_{2} \mathrm{~S}$ NPs were successfully grown in situ on the surface of TGA modified $\mathrm{BiOBr}$ microspheres. (b) The electron-transfer mechanism of a PEC-based $\mathrm{BiOBr} / \mathrm{Ag}_{2} \mathrm{~S}$ immunosensor in PBS electrolyte containing AA. (c) The photocurrent response curves of the PEC-based $\mathrm{BiOBr} / \mathrm{Ag}_{2} \mathrm{~S}$ immunosensor for the determination of insulin at various concentrations. Adapted and reprinted with permission from ref. 457, Copyright 2017.

absorption of $\mathrm{BiOBr} / \mathrm{Ag}_{2} \mathrm{~S}$. The specific binding between insulin present in the samples and the anti-insulin antibody present in the electrode resulted in a linear decrease in the photocurrent intensity with an increase in the amount of insulin in the sample. The composite PEC sensor of $\mathrm{BiOBr} / \mathrm{Ag}_{2} \mathrm{~S}$ revealed its facile preparation, high sensitivity (detection limit of $0.2 \mathrm{pg} \mathrm{mL}^{-1}$ ), reproducibility and perfect stability. A similar concept was also proposed to detect squamous cell carcinoma antigens (SCCAs) using a novel PEC immunosensor based on a $\mathrm{BiOBr} / \mathrm{Bi}_{2} \mathrm{~S}_{3}$ composite. ${ }^{464} \mathrm{Bi}_{2} \mathrm{~S}_{3}$ NPs were synthesized on $\mathrm{BiOBr}$ microstructures via a facile reaction between hydrothermally synthesized $\mathrm{BiOBr}$ and $\mathrm{S}^{2-}$ ions. Similar to the previous example, this composite showed favorable PEC activity when AA was used as an electron donor; however, its photocurrent intensity was $c a .30$ times higher than that of pure $\mathrm{BiOBr}$, resulting in the ultrasensitive determination of SCCA (Fig. 24c). Both of the above examples used BiOBr because of its excellent photoelectric activity, high chemical stability, and wide band gap that can inhibit the recombination of electron-holes $\left(\mathrm{e}^{-} / \mathrm{h}^{+}\right)$ and strengthen the photoelectric activity. ${ }^{465-467}$ Nevertheless, the latter carries the benefits of the high valence band (VB) and conduction band (CB) of $\mathrm{Bi}_{2} \mathrm{~S}_{3}$, which subsequently accelerate electron transfer under visible light irradiation and improves the conductivities of the PEC biosensors. It is worth pointing out that while BiOBr-based PEC detection of tetracycline, $\mathrm{H}_{2} \mathrm{O}_{2}$, glucose, AA, and ciprofloxacin have been recently reported, ${ }^{468-470}$ the use of $\mathrm{BiO}_{x}$ for biosensing is still in its infancy and there is a long way to go and much novel exploration for researchers to fabricate highly sensitive sensors for use in biomedical applications.

\section{2. $\mathrm{Bi}_{2} \mathrm{Se}_{3}$ for biosensing}

As a three-dimensional topological insulator (TI), $\mathrm{Bi}_{2} \mathrm{Se}_{3}$ has shown advantageous properties for biosensing, because of its photothermal conversion ability, ${ }^{471}$ thermoelectric behavior, ${ }^{472}$ Dirac plasmons (oscillations of electrons) ${ }^{473}$ and distinctive electrical conductivity (n-type conductivity, with an energy band gap of $220 \mathrm{meV})^{474}$ that result from the existence of a singlesurface Dirac cone and a six-valley degeneracy in its structure. ${ }^{475}$ Strong spin-orbit coupling of $\mathrm{Bi}_{2} \mathrm{Se}_{3}$ induces nontrivial surface states, which are completely protected against any time reversal 
perturbations, such as nonmagnetic impurities ${ }^{476}$ and crystal defects. In addition, $\mathrm{Bi}_{2} \mathrm{Se}_{3}$ possesses electrochemical catalytic properties, ${ }^{477}$ yielding a highly effective platform for the fabrication of electrochemical biosensors with reduced background signal and increased reproducibility through the suppression of the fluctuation of the spin-polarized charge that is carried on the surface states. ${ }^{478,479}$

Since rapid and selective detection of hydrogen peroxide $\left(\mathrm{H}_{2} \mathrm{O}_{2}\right)$ is in high demand, as it is an important biomarker of the major reactive oxygen species associated with biological disorders, Mohammadniaei et $a l^{480}$ developed an electrochemical biosensor for its detection based on $\mathrm{Bi}_{2} \mathrm{Se}_{3} \mathrm{NPs}$ sandwiched between a gold electrode and a thin layer of thermally-evaporated $\mathrm{Au}\left(\mathrm{Bi}_{2} \mathrm{Se}_{3} @ \mathrm{Au}\right)$. Fig. 25 shows that the encapsulation by the $\mathrm{Au}$ thin layer kept the $\mathrm{Bi}_{2} \mathrm{Se}_{3}$ surface protected, preventing its dissolution into the electrolyte, improving its electrochemical-signal stability, and allowing metallic DNA (mDNA) to be immobilized onto the substrate through $\mathrm{Au}$-thiol interactions and the formation of $\mathrm{Bi}_{2} \mathrm{Se}_{3}$ @ Au-mDNA. The presence of mDNA, consisting of optimized eight- $\mathrm{Ag}^{+}$-inserted dsDNA, rendered a discrete electrochemical signal as a result of the $\mathrm{H}_{2} \mathrm{O}_{2}$ reduction due to the reversible conversion of $\mathrm{Ag}^{2+}$ to $\mathrm{Ag}^{+}$, while $\mathrm{Bi}_{2} \mathrm{Se}_{3}$ acted as an electrochemical signal booster. As a result of the sharp redox signals of the $\mathrm{Ag}^{+}$ion and the low difference between the anodic and cathodic peak potentials $\left(\Delta E_{\mathrm{p}}\right)$ of $66 \mathrm{mV}$ that resulted in a high electron-transfer rate across the structures, as well as increased charge mobility between the interface by the $\mathrm{Bi}_{2} \mathrm{Se}_{3} \mathrm{NPs}$, a fast current response was observed. The developed biosensor showed a remarkable performance to discriminate between MCF-7 and MDA-MB-231 breast cancer subtypes through the ultrafast measurement (1.6 s) of $\mathrm{H}_{2} \mathrm{O}_{2}$ generation produced by each cell line upon exposure to phorbol 12-myristate 13-acetate (PMA) and with a very low detection limit of $10 \times 10^{-9} \mathrm{M} \cdot{ }^{480}$ Fan et al. ${ }^{25}$ also
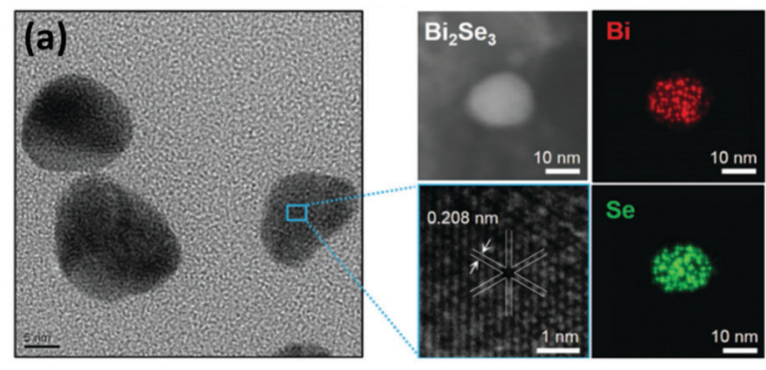

(b)

Centrifuge Self Assembly of
$\mathrm{Bi}_{2} \mathrm{Se}_{3}$ Nanoparticles on $\mathrm{Au}$ Electrode

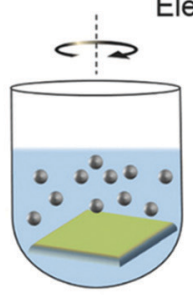

$\mathrm{Au}$

Deposition
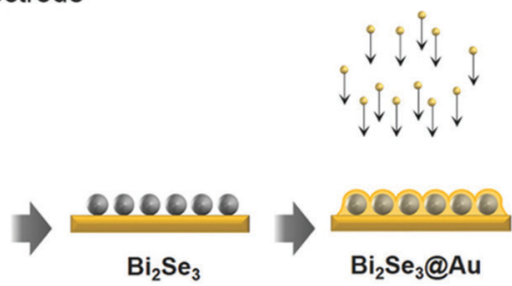

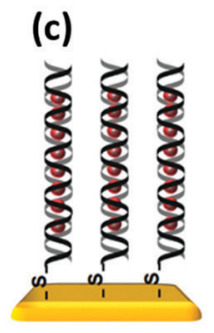

Bare gold-mDNA

I

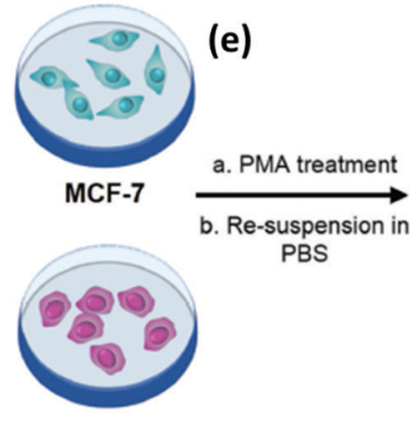

MDA-MB-231

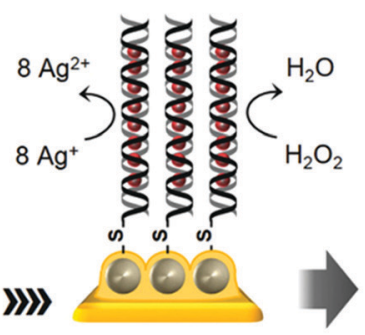

$\mathrm{Bi}_{2} \mathrm{Se}_{3} @ \mathrm{Au}-\mathrm{mDNA}$

II (d)

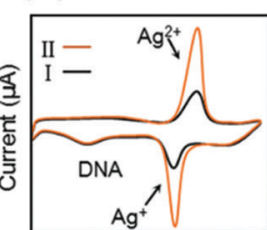

Potential ( $(\mathrm{r})$

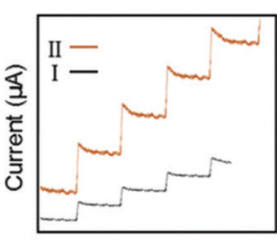

Time (S) Enhancement
Electrochemical Signal / Sensitivity

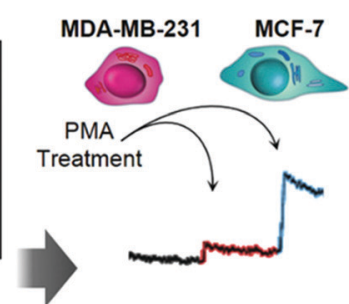

Breast Cancer Cell Identification

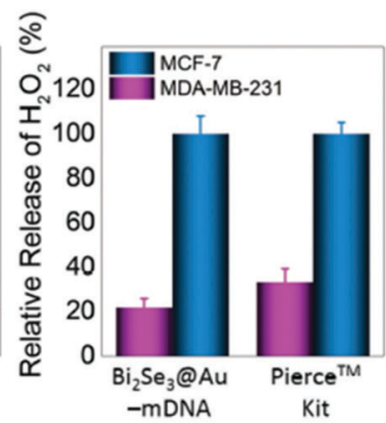

Fig. 25 (a) A TEM image of $\mathrm{Bi}_{2} \mathrm{Se}_{3} \mathrm{NPs}$ and their elemental mapping images obtained from energy-dispersive X-ray spectroscopy (EDS) analysis of the NPs. (b-d) A schematic illustration of the fabrication process of the $\mathrm{Bi}_{2} \mathrm{Se}_{3} @ \mathrm{~A} \mathrm{Au}-\mathrm{mDNA}$ electrode, and its electrochemical behavior for the detection of $\mathrm{H}_{2} \mathrm{O}_{2}$ released from cancer cells. (e) Experimental results from $\mathrm{H}_{2} \mathrm{O}_{2}$ detection via the typical amperometric response recorded at a Bi $\mathrm{Se}_{3}(\mathrm{a} A u-m D N A$ biosensor after the addition of $10 \mu \mathrm{L}$ of a solution of PMA-induced MDA-MB-231 (violate), PMA-induced MCF-7 (blue), and PMA-induced medium (red). The comparison of the $\mathrm{Bi}_{2} \mathrm{Se}_{3} \mathrm{CAu}-\mathrm{mDNA}$ electrode with a Pierce kit demonstrates the reliable measurement of $\mathrm{H}_{2} \mathrm{O}_{2}$ using the developed $\mathrm{Bi}$ based biosensor. Adapted and reprinted with permission from ref. 480, Copyright 2018. 
used $\mathrm{Bi}_{2} \mathrm{Se}_{3}$ NPs to fabricate an electrochemical hemoglobin (Hb) biosensor by immobilizing $\mathrm{Hb}$ on the surface of $\mathrm{Bi}_{2} \mathrm{Se}_{3} \mathrm{NPs}$, promoting the direct electron transfer of $\mathrm{Hb}$, while retaining its biological activity and high catalytic activity for the reduction of $\mathrm{H}_{2} \mathrm{O}_{2}$.

\subsection{Biosensors based on $\mathrm{Bi}_{2} \mathrm{~S}_{3}$ NPs}

$\mathrm{Bi}_{2} \mathrm{~S}_{3}$ is another important type of n-type semiconducting chalcogenide material, recently proposed for the development of biosensors. ${ }^{481,482} \mathrm{Bi}_{2} \mathrm{~S}_{3}$ presents a broad absorption overlapping with the near-IR and visible regions along with a tunable band gap of between 1.3 and $1.7 \mathrm{eV}$, which gives it the benefit of being able to absorb visible light over a wide wavelength range. ${ }^{483}$ Therefore, $\mathrm{Bi}_{2} \mathrm{~S}_{3}$ can act as a light-harvesting substrate for the development of PEC biosensors due to its high absorption coefficient and high incident photon-to-electron conversion efficiency. ${ }^{483,484}$ Nanotubes, ${ }^{485}$ nanorods, ${ }^{444,486,487}$ and nanosheets of $\mathrm{Bi}_{2} \mathrm{~S}_{3},{ }^{488}$ as well as $\mathrm{Bi}_{2} \mathrm{~S}_{3} @ \mathrm{MoS}_{2}$ nanoflowers ${ }^{489}$ are examples of developed photoactive PEC nanosensors for the detection of various analytes, such as methylated DNA, ${ }^{444}$ microRNA, ${ }^{489}$ amyloid- $\beta$ proteins, ${ }^{487}$ avian leucosis virus ${ }^{490}$ and sulfadimethoxine. ${ }^{491,492}$ For the avian leucosis virus (ALVs-J), for example, Sun et al. ${ }^{490}$ used $\mathrm{Bi}_{2} \mathrm{~S}_{3}$ nanorods as a photosensitive layer on top of an ITO electrode to construct an immunosandwich-based biosensor for the PEC detection of this virus. The electrode was constructed by coating an ITO slice with $\mathrm{Bi}_{2} \mathrm{~S}_{3}$ nanorods, followed by a chitosan coating, attachment of an ALVs-J capturing antibody on the chitosan layer, and finally BSA based surface blocking to avoid unspecific binding. Samples containing increasing concentrations of ALVs-J were exposed to the electrode, upon which a detection antibody conjugated to the surface of gold NPs was added to form an immunosandwich complex with the viruses captured on the surface of the antibody modified electrode. Alkaline phosphatase (ALP) was conjugated on the surface of the AuNPs to catalyze the hydrolysis of AAP to AA in situ for the donation of electrons, which enhances the photocurrent of the biosensor. The fabricated biosensor demonstrated a detection limit of $10^{2.08}$ tissue culture infective dose $50\left(\mathrm{TCID}_{50}\right)$.

One way to improve the sensing of Bi-based electrodes is to combine BiNPs with other nanomaterials to form nanocomposites. In recent work, Wang et al. ${ }^{493}$ introduced an innovative enzyme-based PEC biosensor based on a dual signal quenching strategy via the new concept of AA oxidase (AAO)-AA bioeventbased electron donor consumption coupled with the steric hindrance of silicon dioxide $\left(\mathrm{SiO}_{2}\right)$ for the detection of carcinoembryonic antigen (CEA). Ternary hybrid $\mathrm{Bi}_{2} \mathrm{~S}_{3} / \mathrm{Ag}_{2} \mathrm{~S} / \mathrm{TiO}_{2}$ nanotube (NT) arrays were prepared and used as a photoelectrode, as shown in Fig. 26a1. When the AuNPs were decorated on the electrode array, the photocurrent was boosted significantly due to the surface plasmon resonance of the AuNPs. After the conjugation of an aptamer (Apt)-SiO $\mathrm{S}_{2}-\mathrm{AAO}$, the $\mathrm{PEC}$ signal obviously decreased due to the dual quenching effect originating from the enzymatic reaction between AAO and AA that hampers the exposure of the surface of the electrode to the AA and also the steric hindrance of the $\mathrm{SiO}_{2}$. In the presence of CEA, the PEC response was remarkably increased owing to the competition between the CEA and SH-DNA in binding to the Apt of Apt-SiO ${ }_{2}-\mathrm{AAO}$, resulting in the separation of some of the Apt-SiO ${ }_{2}-\mathrm{AAO}$ and increased PEC signal due to the reduced quenching effect. This study also revealed that ternary $\mathrm{Bi}_{2} \mathrm{~S}_{3} / \mathrm{Ag}_{2} \mathrm{~S} / \mathrm{TiO}_{2}$ NTs have better PEC performance compared to $\mathrm{TiO}_{2}$ NTs only or binary $\mathrm{Ag}_{2} \mathrm{~S} / \mathrm{TiO}_{2}$ NTs and $\mathrm{Bi}_{2} \mathrm{~S}_{3} / \mathrm{TiO}_{2}$ NTs (Fig. 26a2). As a result of their wide band gap, $\mathrm{TiO}_{2} \mathrm{NTs}$ possess very poor photoelectric conversion efficiency, which results in a low photocurrent response. Assembly of $\mathrm{Ag}_{2} \mathrm{~S}$ on $\mathrm{TiO}_{2}$ causes a tiny change in the PEC response, but the growth of $\mathrm{Bi}_{2} \mathrm{~S}_{3}$ on the $\mathrm{Ag}_{2} \mathrm{~S} / \mathrm{TiO}_{2}$ NTs resulted in a great increase in the photocurrent intensity. This observation was ascribed to the facile transfer of photogenerated electrons from the conduction band (CB) of $\mathrm{Bi}_{2} \mathrm{~S}_{3}$ to the $\mathrm{CB}$ of $\mathrm{Ag}_{2} \mathrm{~S}$ and then to the $\mathrm{CB}$ of $\mathrm{TiO}_{2}$, consequently promoting the effective separation of electron-hole pairs and photocurrent enhancement. In the future, such novel concepts can be expanded to achieve promising photoactive biosensors, which can meet the challenging questions in the field of PECbased biochemical analysis for the detection of other proteins.

While there are plenty of materials with appropriate electrical conductivity, there are major disadvantages that hinder their wide usage in biosensing. For example, conductive polymers like polyaniline (PANI) suffer from inferior electrochemical stability, weak resistance to acidity variation and poor mechanical properties. ${ }^{494,495}$ To circumvent these weaknesses, $\mathrm{Bi}_{2} \mathrm{~S}_{3}$ NPs can be used to form composites that can make new strides as active materials in the development of biomedical sensors. Zhu et al. ${ }^{496}$ fabricated novel PANI-Bi ${ }_{2} \mathrm{~S}_{3}$ nanocomposite through the potentiodynamic deposition of an ionic liquidcarbon paste electrode (IL-CPE) modified with $\mathrm{Bi}_{2} \mathrm{~S}_{3}$ nanorods in an aniline solution (Fig. 26b1-b3), suggested as an immobilization platform for oligonucleotides to develop impedimetric DNA biosensors for the electrochemical detection of biomolecules. As shown in Fig. 26b4, protonated aniline monomers first adsorb onto the surface of the nanorods via an electrostatic interaction between $\mathrm{NH}_{3}{ }^{+}$and $\mathrm{S}^{2-}$ and coordination of $\mathrm{NH}_{2}$ with $\mathrm{Bi}^{3+}$. Electrochemical scanning then initiates the polymerization reaction of the aniline molecules and $\mathrm{Bi}_{2} \mathrm{~S}_{3}$ contributes to the polymerization of aniline as an excellent electron transfer agent. The results showed that negatively charged DNA probes tightly adsorbed onto the surface of the modified electrodes while maintaining their hybridization activity and identifying potential target DNA with a detection limit of $4.37 \times 10^{-16} \mathrm{M}$. Excellent electrical conductivity, large surface area, facile fabrication, reproducibility, high stability, and good biocompatibility were the main advantages of the devolved biosensor.

\subsection{Bi nanocrystals and Bi film electrodes (BiFEs) for biosensing}

In addition to the abovementioned Bi-based nanomaterials, $\mathrm{Bi}$ by itself can also form semimetal nanocrystals with distinctive properties, such as large Fermi wavelengths, high magnetoresistance and strong diamagnetism. ${ }^{497}$ As a result of its small effective carrier mass, a semimetal-to-semiconductor transition is expected in nanocrystal forms of $\mathrm{Bi}^{498,499}$ a property which is rarely applied in the fabrication of biosensors. Recently, Liu et al. ${ }^{456}$ 


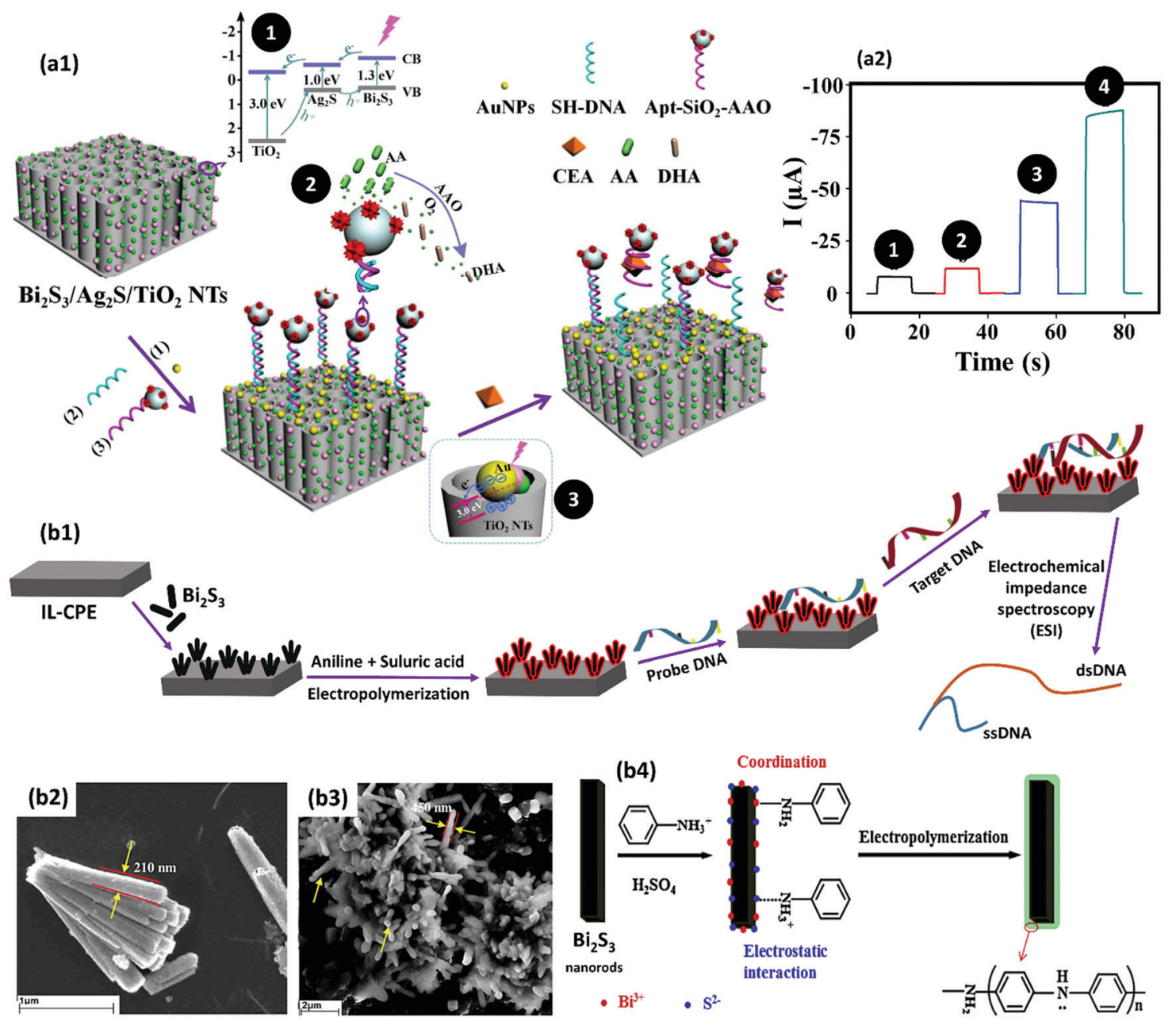

Fig. 26 (a1) A schematic illustration of PEC-based CEA detection using a $\mathrm{Bi}_{2} \mathrm{~S}_{3} / \mathrm{Ag}_{2} \mathrm{~S} / \mathrm{TiO}_{2}$ platform. Insert 1: a diagram showing the energy band levels of the $\mathrm{Bi}_{2} \mathrm{~S}_{3} / \mathrm{Ag}_{2} \mathrm{~S} / \mathrm{TiO}_{2}$ composite and the possible electron transfer process among the energy bands. Insert 2: dehydroascorbic acid (DHA) is produced through the catalytic effect of AAO on AA oxidation. Insert 3: the localized surface plasmon resonance of AuNPs. (a2) PEC signals obtained with (1) TiO 2 NTs, (2) $\mathrm{Ag}_{2} \mathrm{~S} / \mathrm{TiO}_{2} \mathrm{NTs}$, (3) $\mathrm{Bi}_{2} \mathrm{~S}_{3} / \mathrm{TiO}_{2} \mathrm{NTs}$ and (4) $\mathrm{Bi}_{2} \mathrm{~S}_{3} / \mathrm{Ag}_{2} \mathrm{~S} / \mathrm{TiO} \mathrm{O}_{2} \mathrm{NTs}$. Adapted and reprinted with permission from ref. 493 , Copyright 2018. (b1) A schematic illustration of the electrochemical preparation of a PANI- $\mathrm{Bi}_{2} \mathrm{~S}_{3}$ nanocomposite on IL-CPE and its application in an impedimetric DNA biosensor. (b2 and b3) SEM images of the synthesized $\mathrm{Bi}_{2} \mathrm{~S}_{3}$ nanorods and PANI coated $\mathrm{Bi}_{2} \mathrm{~S}_{3}$ nanorods. The diameter of the nanorods increased from 210 to $450 \mathrm{~nm}$ after nanocomposite formation, confirming the uniform surface decoration of the $\mathrm{Bi}_{2} \mathrm{~S}_{3}$ nanorods with PANI. (b4) A schematic representation of PANI growth on $\mathrm{Bi}_{2} \mathrm{~S}_{3}$ via electropolymerization. Adapted and reprinted with permission from ref. 496, Copyright 2015.

developed a novel core-shell nanohybrid ( $\mathrm{NH}$ ), in which $\mathrm{Bi}$ nanocrystals were encapsulated within a heteroatom N,Ocodoped nanocarbon (NOC) ultrathin carbon shell (Bi@NOC) as a PEC aptasensor for the early clinical diagnosis of cancer via the detection of telomerase activity in cells (Fig. 27a). The coreshell $\mathrm{NH}$ was obtained by the reduction of $\mathrm{Bi}(\mathrm{Ac})_{3}$ in the presence of oleylamine to generate $\mathrm{Bi}$ nanocrystal cores that can catalyze the carbonization of folic acid to form NOC on the surface of the core. Bi nanocrystals are recognized as a semiconductor with a small band gap and unique potential to generate weak photocurrent upon excitation by visible light. ${ }^{500}$ While the NOC shell endows the system with high compatibility as it is derived from folic acid, it can also boost the interfacial electron transfer rate and inhibit the electron-hole recombination in the Bi nanocrystal core, because of its high charge carrier mobility and super electron accepting ability. ${ }^{501}$ The combination of all these criteria leads to the enhanced photoelectric conversion efficiency and photocurrent intensity of the biosensor. In addition, the Fermi level alignment of the core and the shell favorably accelerate the generation of a photocurrent through the separation of photogenerated charge carriers, endowing the Bi@NOC aptasensor with excellent PEC activity under the irradiation of visible light. Thioflavin-T (Th-T), a fluorescent dye capable of binding to telomere sequences in a G-quadruplex manner, ${ }^{502}$ was added to the biosensor as a telomerase-recognizing photoactive reagent. AA was also used as an electron donor. The Bi@NOC biosensor linearly and reproducibly detected telomerase activity with a high sensitivity and low detection limit of only 60 cells. ${ }^{456}$ Although there are not a plethora of studies on nanocrystalline $\mathrm{Bi}$ biosensors, the abovementioned features demonstrate their potential application in the diagnosis of clinically relevant pathologies, such as cancer. 

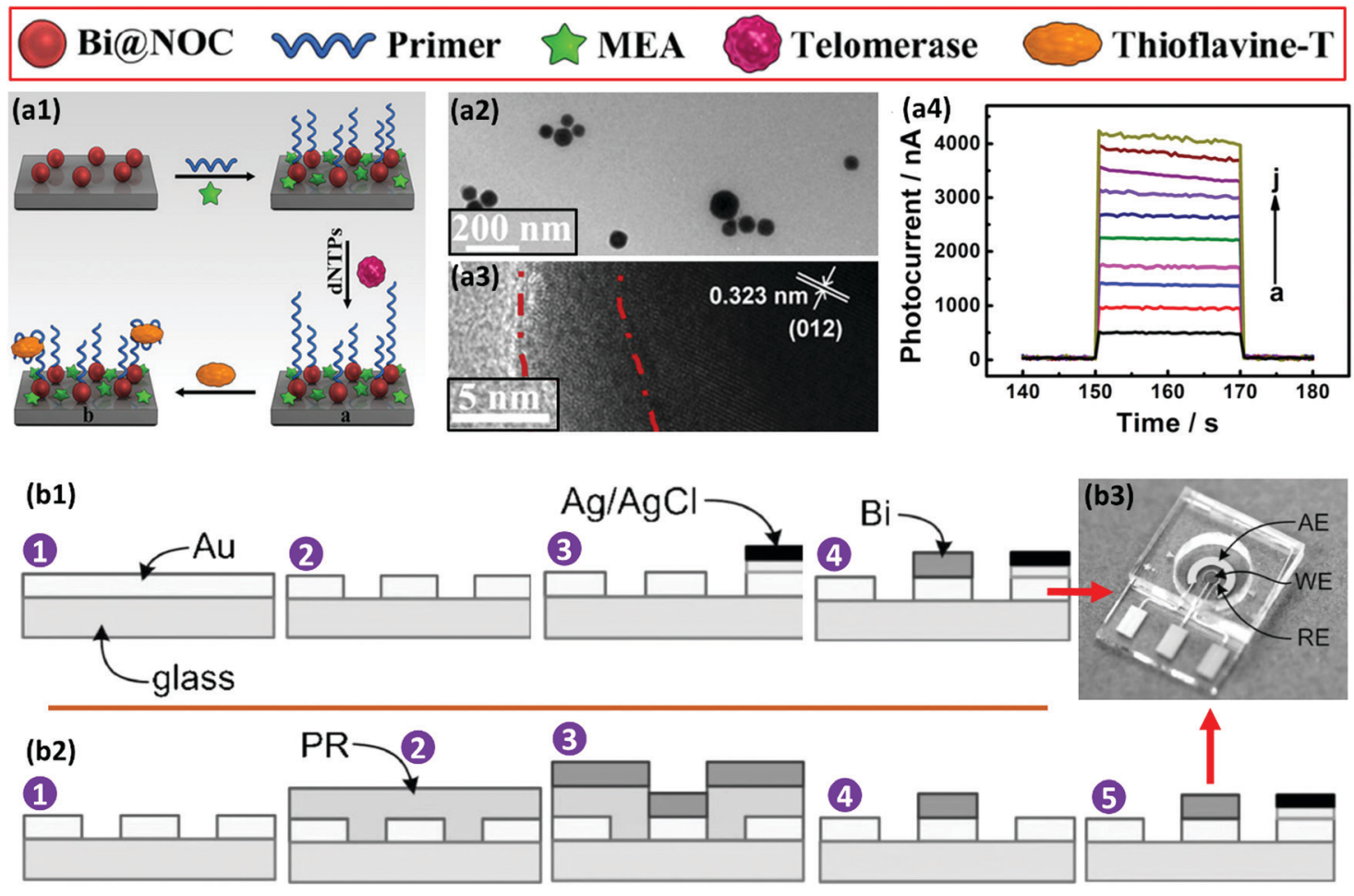

Fig. 27 (a1) The schematically depicted fabrication process of the sensor for the telomerase detection via the PEC activity of Bi@NOC NH and the telomerase-related signal amplification effect of Th-T. MEA is an abbreviation of monoethanolamine. (a2 and a3) TEM images of the obtained Bi@NOC nanostructure at low (top) and high (bottom) magnification. The shell is shown between the red lines. (a4) The photocurrent intensity of the Bi@NOC NH biosensor in response to increasing concentrations of cells from (a) $5 \times 10^{2}$ to (j) $1 \times 10^{6}$ cells per mL. Adapted and reprinted with permission from ref. 456, Copyright 2018. The sequence of steps for the fabrication of (b1) an electrodeposited Bi WE sensor and (b2) an evaporated Bi WE sensor. (b3) A close-up of the completed electrochemical sensor cell. Adapted and reprinted with permission from ref. 524, Copyright 2013.

The electrical, optical and structural characteristics of $\mathrm{Bi}$ have also made it desirable to fabricate BiFEs for biosensing. ${ }^{503}$ Strategies for designing BiFEs include: electrochemical deposition of Bi precursors; ${ }^{504,505}$ stripping voltammetry where a soluble $\mathrm{Bi}^{3+}$ salt is added to a sample solution and co-deposited with a target analyte $;^{506}$ and the incorporation of insoluble forms of $\mathrm{Bi}\left(\mathrm{Bi}_{2} \mathrm{O}_{3}\right.$ or $\mathrm{BiPO}_{4}$ ) into an ink for screen-printed electrode manufacturing further associated with electrochemical deposition for the formation of a thin film on the electrode surface. ${ }^{507}$ BiEF electrode configurations are usually made by in situ and ex situ reduction of $\mathrm{Bi}^{3+}$ ions and their subsequent deposition on the surface of electrode substrates to form a film at nanoscale. ${ }^{508,509}$ Compared to carbon and mercury film electrodes, BiFEs are economically viable, can be deposited in various types of substrates, generally possess high mechanical stability, are biocompatible and environmentally friendly. The ability to form alloys with different metals, simple preparation, well defined signals and partial insensitivity to dissolved oxygen are also other benefits of BiFEs, similar to those of mercury film electrodes. ${ }^{510} \mathrm{Bi}$ compounds have been used to modify different carbonaceous electrode materials, such as carbon pastes, ${ }^{511}$ graphite-epoxy composites, ${ }^{512}$ borondoped diamond, ${ }^{513}$ carbon films, ${ }^{514}$ glassy carbon, ${ }^{431}$ screenprinted carbon inks, ${ }^{515}$ or pencil graphite. ${ }^{516} \mathrm{Bi}$ has also been deposited in combination with polymers, ${ }^{517,518}$ and on metallic electrodes, such as $\mathrm{Au}, \mathrm{Cu}$, or $\mathrm{Pb} .{ }^{449}$
Studies have shown that the incorporation of Bi NPs onto an electrode surface is more attractive compared to pre-plating the BiFEs on the electrode. ${ }^{519,520}$ This is due to the fact that the sensor characteristics, such as the sensitivity and detection limit of the BiNP modified electrodes, are superior to those of the BiFEs. ${ }^{521,522}$ In addition, BiNPs also showed superior properties compared to those of BiFE, such as better biocompatibility, electrocatalytic activity, good conductivity, and larger active surface area coupled with an enhanced mass transfer effect, properties that generally improve the sensitivity and detection limit of biosensors. For example, Nigovic et al. ${ }^{521}$ modified the surface of a glassy carbon electrode (GCE) with a nanocomposite combination of BiNPs and carbon nanotubes (CNTs) to form a BiNP-CNT/GCE sensor for the quantification of the drug sulfasalazine in human serum samples. This sensor indicated superior electroanalytical performance for drug determination in comparison to BiFE. However, BiFE also presented advantages over the BiNP-CNT nanocomposite, such as its favorable surface stability and the possibility of performing successive voltammetric measurements without time-consuming surface cleaning, while the nanocomposite required the renewal of the electrode surface after each experiment to achieve reproducible measurements. ${ }^{521,522}$ In general, BiNPs are more attractive for use in biosensing technology; however, the abovementioned advantages of BiFEs have resulted in their usage in a wide range 
of interesting environmental and clinical applications, such as trace metal detection ${ }^{431}$ and for the detection and measurement of different compounds, such as aminosalycilate drugs, ${ }^{522}$ progesterone, ${ }^{510}$ glucose,${ }^{523}$ zinc $^{524}$ and vitamin B2. ${ }^{525}$ As a result of the limitations of the bed-side monitoring of zinc ( $\mathrm{Zn})$ in biological samples due to the requirement of expensive instruments and expert personnel, Kang et al. ${ }^{524}$ reported the fabrication of a lab-on-a-chip BiFE sensor for detection using square wave anodic stripping voltammetry. As shown in Fig. $27 \mathrm{~b}$, the biosensor is composed of a Bi working electrode (WE), an integrated silver/silver chloride $(\mathrm{Ag} / \mathrm{AgCl})$ reference electrode $(\mathrm{RE})$, and a gold auxiliary electrode (AE). The detection limit of the sensor in $0.1 \mathrm{M}$ of acetate buffer at $\mathrm{pH} 6$ was $60 \mathrm{nM}$, with rapid response, high accuracy at low concentrations of $\mathrm{Zn}$, the requirement of only a small sample volume, and reduced cost among the main advantages of the developed sensor. The electrodeposited Bi WE and evaporated Bi WE were fabricated using two different protocols. For the electrodeposited Bi WE, a single evaporation step was applied to deposit a gold metal layer, and then, single photolithography patterning and electrodeposition of Bi were performed (Fig. 27b1). As for the evaporated Bi WE process, the final device was achieved by: (1) deposition of the gold metal layer via an evaporation step; (2) first photolithography patterning to define the shape of the electrode; (3) second photolithography step to define the sacrificial resist mask; (4) an e-beam evaporation step to deposit a thin film of $\mathrm{Bi}$; and (5) finally, a lift-off step to pattern the Bi WE (Fig. 27b2). Using the lab-on-a-chip BiFE sensor shown in Fig. 27b3, Zn was successfully detected in serum samples by complementary atomic absorption spectroscopy, demonstrating the potential for the future development of portable BiFE-based biosensors for the detection of biological analytes.

In this section, we discussed the most relevant properties and described some of the latest advancements verified throughout the last years in the application of $\mathrm{Bi}$ in biosensing technology. Significant development has been achieved at a fast pace in biosensing using $\mathrm{Bi}$, allowing the replacement of toxic metals, such as mercury in sensing devices, and direct measurements in physiologically relevant samples or fluids. We believe that in the future, BiNP-based biosensors may take up a relevant position in the field of biosensing, as the advantages of their use largely outweigh the drawbacks. Therefore, we envisage that the present fast-paced development of Bi-based biosensors will bring considerable significance to biomedical applications in the near future.

\section{Bi-Based biomaterials for tissue engineering and implantation}

Tissue engineering can be described as the capability to design spatial and temporal structures to engender a favorable, biologically inspired environment for scaffold matrices to fill tissue voids by cell adhesion, migration, proliferation, and differentiation. ${ }^{526}$ The advent of this interdisciplinary field has evolved towards the innovative design and fabrication of biomaterials with stringent requirements, to closely mimic the hierarchical constructs of native tissues for regeneration and self-repair. ${ }^{527}$ The synergistic combination of tissue engineering and regenerative medicine has been at the forefront in recent years, as a result of the fabrication of threedimensional (3D) porous architectures with excellent biocompatibility, controlled biodegradation and proper structures that maintain oxygen and nutritional perfusion. ${ }^{528}$ Current efforts have resulted in the introduction of new biomaterials for the regeneration of different tissues, such as skin, ${ }^{529}$ bone, ${ }^{530,531}$ and cartilage. ${ }^{532} \mathrm{Bi}$ is one of the new materials used to devise promising new platforms for biocompatible scaffolding in the field of tissue engineering and regenerative medicine. ${ }^{285,533}$ Therefore, there are very few reports on this application of Bi. Thus, in this section, we will reflect on the emerging advancements of this relatively new area of research and discuss the considerations for further progress on Bi-containing formulations concerning regenerative medical applications.

Bone is a complex type of tissue consisting of cells and hierarchically arranged collagen fibrils, hydroxyapatite and proteoglycans aligned together as a hard structure with very high compressive strength. While bone possesses considerable ability to repair and renew itself, the regeneration process is not quick enough and it is limited in severe bone defects. ${ }^{534-537}$ Harvesting bone autografts to replace large bone defects is a common practice in medicine. Yet, this may lead to a significant risk of morbidity, scarring and deformity, as well as surgical complications, including bleeding, inflammation and infection. ${ }^{538}$ This has encouraged new practices in generating novel therapeutic formulations for bone tissue, providing an alternative to conventional implants. ${ }^{539,540} \mathrm{Bi}$ has been exploited as a new component to be included in bone scaffolds and cements due to its favorable properties, such as biocompatibility, low toxicity and environmentally friendly and stable photothermal effects, advantageous in the therapeutic functionality of a materialsbased formulation for bone regeneration. ${ }^{541}$ In addition, when used as a dopant in bioactive glass (BG), it can enhance the mechanical, biological, and osteogenic properties of the BG. ${ }^{542}$ Radiopacity of the $\mathrm{Bi}$ also facilitates imaging of the treated area using X-rays and CT scanning. ${ }^{541,543}$ For example, bismuth aluminate $\left(\mathrm{Al}_{3} \mathrm{BiO}_{6}\right)$ has been used to increase opacity in calcium phosphate cements, at the same time improving the proliferation of bone marrow stem cells, demonstrating suitability for peri-/post-operative radiographic assessment of cement placement after bone repair procedures and possibly bone regeneration. ${ }^{541,544}$ BiNPs have also recently served as active materials for bone tissue engineering. According to Webster et al., ${ }^{545} \mathrm{Bi}^{3+}$ ions in the replacement of $\mathrm{Ca}^{2+}$ ions in substituted hydroxyapatite, presented better alkaline phosphate activity deposition of calcium-containing mineral, suggesting enhanced osteoblast proliferation, pertinent for both bone and dental applications. As a dopant, Bi improved osteoblast long-term calcium-containing mineral deposition, adhesion, and differentiation, therefore suggesting it to be a great dopant choice for enhancing the properties of HA, leading to a revision of the criteria essential for successful bone prosthetic applications.

BGs have a wide range of clinical applications for the reparation and reconstruction of bone, thus have been a preferential 
biomaterial for modification with Bi composites. ${ }^{543,546,547}$ BGs have the ability to serve as an eluting agent of relevant therapeutics, contributing to therapeutic strategies against diseases such as osteomyelitis and bone cancer, ${ }^{543,548-551}$ particularly when combined with Bi as one the main components. Regarding the treatment of osteomyelitis, BGs by which the addition of $\mathrm{Bi}$ ions renders them with antimicrobial activity and promoted bone regeneration were investigated by Prasad et al. ${ }^{546}$ They demonstrated a combinatorial antibacterial effect and cell proliferation induction due to the combined action of antibacterial $\mathrm{Bi}^{3+}$ ions and an increase in the $\mathrm{pH}$ value of the surrounding medium caused by the BG. ${ }^{552}$ As for bone cancer-related defects, Wang et $a .^{543}$ described a photothermal effect in a Bi-doped germanate BG, which demonstrated a dual function of hyperthermia (to kill lingering bone tumor cells) and bioactive properties for apatite remineralization and tissue repair, allowing the treatment of large bone defects arising from bone tumor therapy. The main mechanisms of action rely on a high photothermal conversion efficiency that allows the effective killing of bone tumor cells upon illumination with NIR light (by inducing thermal apoptosis through heat generated from optical input), as well as its ability to promote osteogenic cell adhesion, proliferation, differentiation, and mineralization. Both in vitro and in vivo data demonstrated promising results, regarding the efficiency of the photothermal treatment in decreasing UMR106 bone tumor cells and tumor volume. ${ }^{543}$ A multiferroic Bi-reinforced BG was also recently developed by Khatua et al. ${ }^{196,553}$ in order to investigate whether $\mathrm{BiFeO}_{3}$ in the $\mathrm{BG}$ can accelerate cellular activities for rapid tissue healing by applying a magnetic field externally to induce in situ electrical polarization. Mouse preosteoblast cells on BG composites under different external magnetic field treatments demonstrated 3-fold enhancement of cell viability and proliferation for these composites at specific magnetic field strengths of 200 or 350 milliTesla (mT), which improved polarization and decreased the degree of crystalline phases of the BG. ${ }^{196}$ These studies are considered to have opened the door for the further investigation of the application of $\mathrm{Bi}$ composites in bone repair.

Dental repair is another area of interest in which to use Bi-based materials. The loss of dental tissue function due to trauma, infection, abnormalities or congenital diseases is a common health care problem worldwide. ${ }^{554}$ Owing to the limited regenerative ability of hard teeth, the demand for a novel therapeutic concept is required to provide an alternative for dental transplantation and implantation. Despite the relatively high success rate of implantation techniques, several factors contribute to their shortcomings, as the need for surgery, very costly nature of the procedures, as well as risk of complications. Novel dental tissue regeneration studies aim to enable cell-to-cell and cell-to-scaffold interactions at the micro level through interconnected pores of scaffolds with adequate mechanical properties and specific surface characterization suitable for dental growth. ${ }^{555,556}$ An ideal filling material for tooth repair is required to offer complete and impervious obturation of the root canal system, sealing the pathways of communication from their surrounding tissues. ${ }^{557}$ Similar to their promising application in bone repair, Bi-based materials, especially $\mathrm{Bi}_{2} \mathrm{O}_{3}$, have shown adequate biocompatibility, cell proliferation and a three-dimensional structure adequate for dental fillings. In addition, they present ideal radiopacity, which is crucial for monitoring various stages of root canal therapy and to enable easy monitoring of the filling of the root canal with endodontic cement. ${ }^{544,558}$ For example, Kuratate et $a .^{533}$ investigated the reparative process of mechanically exposed pulps treated and capped with MTA. MTA is a cement composed of $\mathrm{CaO}, \mathrm{SiO}$, and $\mathrm{Bi}_{2} \mathrm{O}_{3}$, known to stimulate the proliferation of fibroblasts and osteoblast-like cells. MTA can also induce the gene expression of hard tissue-related proteins, such as osteopontin, osteonectin, and osteocalcin in vitro. Treatment with MTA demonstrated high frequency of dentin bridge formation, occurring within 14 days after MTA capping in rat molars. ${ }^{559-562} \mathrm{Bi}_{2} \mathrm{O}_{3}$ was the main component of the MTA that provided its radiopacity. In this study, the capacity of MTA to induce regeneration of hard tissue in dental pulps was demonstrated with proliferation, migration, and differentiation of progenitor cells into new odontoblast-like cells before new matrix secretion at the exposure site. Evidence of the cell proliferation, the appearance of nestin-immunoreactive odontoblast-like cells, and deposition of osteopontin, a noncollagenous protein involved in matrix mineralization, were verified as potential signs of dental regeneration. Similarly, to develop an ideal biomaterial for orthopedic and dental tissues, Pazarçeviren et al. ${ }^{547}$ prepared a Bi-doped nanobioactive bioglass (nBG) using a sol-gel method and then made a composite by adding graphene oxide (GO). The presence of Bi resulted in improved biocompatibility, increased diametral tensile strength, and efficient cell spreading on the composite. In the above studies, the role of $\mathrm{Bi}$ on the reparative/ regenerative processes was not studied in detail, but rather the composites as a whole. Therefore, further studies are required in order to understand the real role of $\mathrm{Bi}$ in the processes of dental repair and/or regeneration.

These few examples of bone and dental repair by Bi-based biomaterials demonstrate the infancy of the field and very early stages of the investigation on the characterization and in vitro assessment of such Bi-based composites, where the specific role of the Bi component is not specifically or thoroughly understood in the context of cellular repair. We believe that tissue engineering research is slowly developing in this direction, which will be fulfilled with more detailed studies in the coming years.

\section{Conclusions and future perspectives}

Although in the last few years the research has increasing on BiNPs due to their unique multifunctional potential for different biomedical applications, there are still several challenges that hinder their clinical translation, which require further investigations to meet these challenges. One big challenge is the lack of standard protocols to test and compare the biocompatibility of these NPs prepared via different approaches. Different researchers have tested BiNPs, but factors, such as the preparation method, 
functionalization method, particle size, particle charge, composition, shape, surface coating, applied dose, animal model, type of model disease, etc., were different with no standard reference for comparison. For example, $\mathrm{Bi}_{2} \mathrm{O}_{3}$ NPs demonstrated different in vitro cytotoxicity behavior when annealed in Ar and air. ${ }^{563}$ The air-annealed $\mathrm{Bi}_{2} \mathrm{O}_{3}$ NPs generated higher levels of ROS, resulting in higher cell apoptosis and death, while the Ar-annealed ones demonstrated a ROS-scavenging effect. Therefore, it is presumed that the toxicity of BiNPs is dependent on the preparation method, which subsequently affect their particle size and surface chemistry. Therefore, a comprehensive study is needed to establish correlations between the properties of BiNPs, the experimental parameters, and their toxicity in order to reliably discuss the biosafety of these particles. In addition, genotoxicity studies and investigation of the apoptotic pathways affected by BiNPs should be performed in the future for further clarification of the toxicity of BiNPs.

Several studies have demonstrated both the biocompatibility of BiNPs and also their rapid biodegradation in vivo, ${ }^{48,213,222,237,245,259}$ but most of these studies were based on short-term assays, which cannot be expanded to determine the long-term biosafety of BiNPs. Overall, in order to classify BiNPs as "practically nontoxic" materials, a series of systematic biosafety investigations on both small (e.g., zebra fish and mice) and large animal models (e.g., pigs, dogs, and monkeys) are critical for understanding their acute toxicity on various biosystems (e.g., neurotoxicity, immunotoxicity, etc.) and chronic/long-term side effects on different organs. Further information that is missing is the behavior of BiNPs at the nano-biointerface, where cellular mechanisms involved in the uptake of BiNPs into various types of cells should be studied. The determination of precise mechanisms will allow the development of favorable NPs for efficient therapeutic purposes. Further surface modification of BiNPs with various materials and their effects on immunogenicity, pharmacokinetics, and biodistribution of the particles is another area that is not well explored.

As for the fabrication process of BiNPs, most of the current approaches are based on bottom-up synthesis and efforts have still not shifted to top-down methods. Hence, more attempts should be devoted in this area to explore morphology and surface termination controllable top-down procedures. Additionally, the marriage of BiNPs with other functional materials should be investigated further to fabricate hybrid nanostructures that combine the advantages of both for multifunctional biomedical applications. The fabrication of ultrasmall Bi-based quantum dots and their usage in biomedicine also needs further attention. ${ }^{564-566}$ Similar to other types of quantum dots, the attractive luminescence properties of $\mathrm{Bi}$ quantum dots can be applied in diagnostic medicine. Nevertheless, the synthesis of these ultra-small particles is quite challenging. One strategy, which is worth testing, might be through the filtration of big particles after sequential rounds of centrifugation. Moreover, further novel studies on Bi nanocomposites with the capability of responding to both NIR-I (700-950 nm) and NIR-II (1000-1700 nm) windows is beneficial for deep-tissue applications and would direct the design of
BiNPs with enhanced PTT efficiency. This property will also allow the design of novel theranostics through the PAI and PTT of deep tissues.

Tuning of the optical and electrical properties of BiNPs via the doping of various elements on their surface or alteration of surface termination is another area of exploration to create highly sensitive Bi-based biosensors. It is noted that while the development of BiNPs in nanomedicine is still in its infancy, emerging trends are expected to shift from the current focus on antibacterial therapeutics or cancer therapy toward the exploitations of their potential in the design of wearable, as well as lab-on-a-chip biosensors with desirable multifunctionality, programmability and biocompatibility. This specific application of BiNPs is achievable by designing multicomponent composites and developing ground-breaking fabrication strategies. The catalytic properties of BiNPs have recently attracted attention in biosensing, because of their possible function in catalyzing specific biological reactions. The exploration of new scenarios in this field is a research hotspot, which needs further clarification in the future studies on BiNPs. In addition, the potential benefits of BiNPs should be further explored for stem-cell engineering through thermo-modulation, gene therapy, active targeting of different organs within the body rather than just cancer cells, regenerative medicine, development of novel hydrogel based 3D-cell cultures, containing BiNPs for thermal or electrical signaling of the encapsulated cells, preparation of injectable thermoresponsive hydrogels, and thermally or electrically induced re-programming of immune cells (see the detailed perspectives in Fig. 28).

This review has distinctly shown the great optimism surrounding the use of BiNPs and their composites as promising platforms for use in different biological and biomedical applications. Bi-based nanomaterials can be engineered with numerous functionalities to achieve specific physical, chemical, and biological properties. The unrivalled advantage of BiNPs is their high versatility, recognized as reproducible nanostructures, which can be applied in diagnostic multi-imaging, as sensitizer for PTT and RT, antibacterial agents, biosensing, and also as tissue regenerative materials. Moreover, compared to many other types of metal-based NPs, the challenges of BiNPs seems to be less in terms of the translation of applications, due to their precise composition/structure control, facile large-scale production, and ease of surface engineering. The low price of BiNPs also adds double value to their other abovementioned benefits and is considered as a very strong driving force for further studies on the scale-up of new Bi-based nanomedicines. Nevertheless, comprehensive and long-term monitoring of their biosafety must be taken into account before BiNPs themselves can flourish as important materials in biomedicine. To do this, we should be aware of the fact that the implementation of toxicity assays by following existing guidelines is compulsory. This is suggested as we expect the continued expansion of research on BiNPs at a rapid rate and the emergence of their novel biomedical applications in the near future.

In summary, the ongoing research progress in the field of BiNPs can lead to exceptional advances in current therapeutic 


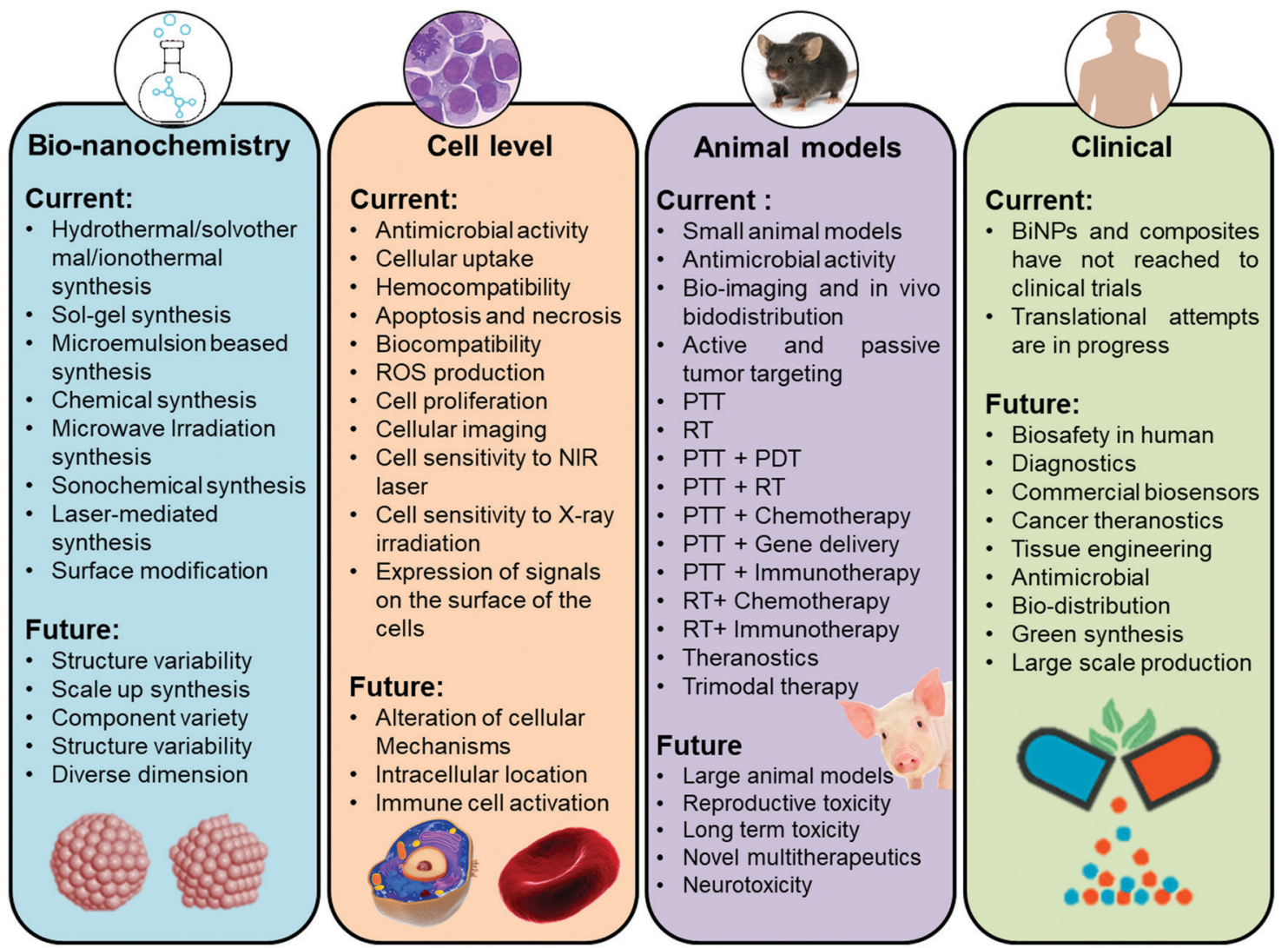

Fig. 28 A summary of the current research developments, conclusions, and future perspectives related to BiNPs and Bi-containing composites for versatile biomedical applications.

methods for cancer by affecting the biology of diseased tissues beyond our imagination. It is our opinion that while highly detailed and time-consuming investigations are needed to move products into the clinical trial phase, BiNPs might cross this path in a fast manner and commercialized products could enter the market in the near future. It is highly expected that multidisciplinary efforts among biologists, chemists, physicists, and engineers will promote a mechanistic understanding of BiNPs and allow the limitations of Bi-based nanomedicines to be pushed, while moving the currently successful paradigms more rapidly toward clinical translation.

\section{List of abbreviations}

$\begin{array}{lll}\mathrm{AAO} & \text { Ascorbic acid oxidase } & \mathrm{Bi} @ \mathrm{Co} @ \mathrm{CN} \text { ) NPs } \\ \mathrm{AE} & \text { Auxiliary electrode } & \mathrm{Bi}_{2} \mathrm{MoO}_{6} \\ \mathrm{AIPH} & \text { 2,2-Azobis[2-(2-imidazolin-2- } & \mathrm{Bi}_{2} \mathrm{O}_{3} \\ & \text { yl)propane]dihydrochloride } & \mathrm{Bi}_{2} \mathrm{~S}_{3} \\ \mathrm{Al}_{3} \mathrm{BiO}_{6} & \text { Bismuth aluminate } & \mathrm{Bi}_{2} \mathrm{Se}_{3} \\ \mathrm{ALP} & \text { Alkaline phosphatase } & \mathrm{Bi}_{2} \mathrm{Te}_{3} \\ \text { ALT } & \text { Alanine aminotransferase } & \mathrm{Bi}_{2} \mathrm{WO}_{6} \\ \text { ALVs-J } & \text { Avian leucosis virus } & \mathrm{BiCl}_{3} \\ \text { AOT } & \text { Bis(2-ethylhexyl)sulfosuccinate } & \mathrm{BiFeO}_{3} \\ \text { APS } & \text { Ammonium persulfate } & \mathrm{BiFEs} \\ \text { APTMS } & \text { (3-Aminopropyl) trimethoxysilane } & \mathrm{BiOBr}\end{array}$

ASTM

$\mathrm{Au}$

AuNPs

$\mathrm{Ba}$

BACP-2

BAL

$\mathrm{BC}$

BET

BG

$\mathrm{Bi}$

BiNPs

$\mathrm{Bi}\left(\mathrm{NO}_{3}\right)_{3} \cdot 5 \mathrm{H}_{2} \mathrm{O}$

$\mathrm{Bi}(\mathrm{OH})_{3}$

$\left(\mathrm{Bi}_{2} \mathrm{O}\right)_{2} \mathrm{CO}_{3}$

$\mathrm{Bi} @ \mathrm{Co} @ \mathrm{CN}) \mathrm{NPs}$

$\mathrm{Bi}_{2} \mathrm{MoO}_{6}$

$\mathrm{Bi}_{2} \mathrm{O}_{3}$

$\mathrm{Bi}_{2} \mathrm{~S}_{3}$

$\mathrm{Bi}_{2} \mathrm{Te}_{3}$

$\mathrm{Bi}_{2} \mathrm{WO}_{6}$

$\mathrm{BiCl}_{3}$

BiFEs

Argon

American Society for Testing and Materials Gold

Gold nanoparticles

Barium

Bi-asparagine coordination polymer spheres

2,3-Dimercapto-1-propanol

Bismuth citrate

Brunauer-Emmett-Teller

Bioactive glass

Bismuth

Bismuth-containing nanoparticles

Bismuth nitrate pentahydrate

Bismuth hydroxide

Bismuth oxide carbonate

Nitrogen-rich carbon-coated bismuth/cobalt Bismuth molybdate

Bismuth oxide

Bismuth sulfide

Bismuth selenide

Bismuth telluride

Bismuth tungstate

Bismuth chloride

Bismuth ferrite

Bi film electrodes

Bismuth oxybromide 


\begin{tabular}{|c|c|c|c|}
\hline $\mathrm{BiOBr}$ & Bismuth oxybromide & Gd & Gadolinium \\
\hline $\mathrm{BiOCl}$ & Bismuth oxychloride & Gd & Gadolinium \\
\hline $\mathrm{BiOI}$ & Bismuth iodide oxide & $\mathrm{Ge}$ & Germanium \\
\hline $\mathrm{BiOX}$ & Bismuth oxyhalides & GEL & Gelatin \\
\hline Bi-PEG NCs & Pegylated metallic bismuth nanocrystals & GI & Gastrointestinal \\
\hline $\mathrm{BiPO}_{4}$ & Bismuth phosphate & GLP & Ganoderma lucidum polysaccharide \\
\hline BisBAL & Bismuth dimercaptopropanol & GO & Graphene oxide \\
\hline $\mathrm{BiVO}_{4}$ & Bismuth vanadate & GOD & Glucose oxidase \\
\hline \multirow[t]{2}{*}[\mathrm{BMIM}]{$\mathrm{BF}_{4}$} & 1-n-Butyl-3-methylimidazolium & GSH & Glutathione \\
\hline & tetrafluoroborate & GSH-Px & Glutathione peroxidase \\
\hline BMSNR & Mesoporous silica-coated $\mathrm{Bi}_{2} \mathrm{~S}_{3}$ nanorods & H. pylori & Helicobacter pylori \\
\hline BNRs & Bismuth sulfide nanorods & HA & Hyaluronic acid \\
\hline BP NCs & $\mathrm{Bi}_{2} \mathrm{Se}_{3} @ P E G$ nanocapsules & HA-DS & Hyaluronic acid-dextran sulfate \\
\hline BPDC & $\mathrm{Bi}_{2} \mathrm{Se}_{3} @ p e g /$ dox/ce6 & $\mathrm{Hb}$ & Hemoglobin \\
\hline BSA & Bovine serum albumin & HIF- $1 \alpha$ & Hypoxia-inducible factor \\
\hline BSS-NPs & Bi subsalicylate nanoparticles & HIFU & High-intensity focused ultrasound \\
\hline C. elegans & Caenorhabditis elegans & $\mathrm{HNO}_{3}$ & Nitric acid \\
\hline CAs & Contrast agents & HO-1 & Heme oxygenase \\
\hline $\mathrm{CB}$ & Conduction band & hpf & Hours postfertilization \\
\hline CCL2 & Chemokine ligand 2 & HSA & Human serum albumin \\
\hline CCR2 & Chemokine receptor 2 & HSP & Heat-shock protein \\
\hline Ce6 & Chlorin e6 & i.t. & Intratumorally \\
\hline CEA & Carcinoembryonic antigen & $\operatorname{IgM}$ & Immunoglobulin $\mathbf{M}$ \\
\hline CMC & Carboxymethyl cellulose & IL & Interleukin \\
\hline CNTs & Carbon nanotubes & IL-CPE & Ionic liquid-carbon paste electrode \\
\hline $\mathrm{CPP}$ & Cell penetrating peptide & IRT & Infrared thermography \\
\hline CS & Chitosan & ITO & Indium tin oxide \\
\hline $\mathrm{CT}$ & Computed tomography & IV) & Intravenous \\
\hline CTAB & Cetyltrimethylammonium bromide & $\mathrm{KOH}$ & Potassium hydroxide \\
\hline CTAC & Cetyltrimethylammonium chloride & LA & Lauric acid \\
\hline DCF & $2^{\prime}, 7^{\prime}$-Dichlorofluorescein & L-Cys & L-Cysteine \\
\hline DCFH-DA & $2^{\prime}, 7^{\prime}$-dichlorofluorescin diacetate & MADS & Methacrylated dextran sulfate \\
\hline DEG & Diethylene glycol & MAHA & Methacrylated hyaluronic acid \\
\hline DLPC & 1,2-Dilauroyl-sn-glycero-3-phosphocholine & $\mathrm{MB}$ & Methylene blue \\
\hline DLS & Dynamic light scattering & MDA & 3,4-Methylenedioxyamphetamine \\
\hline DMF & $N, N$-Dimethylformamide & mDNA & Metallic DNA \\
\hline DMF & Dimethylformamide & MDR & Multidrug-resistant \\
\hline DMSO & Dimethyl sulfoxide & MIC & Minimal inhibitory concentration \\
\hline DOX & Doxorubicin & $\mathrm{Mn}$ & Manganese \\
\hline dpf & Days postfertilization & MNs & Microneedles \\
\hline E. coli & Escherichia coli & mPS & Mesoporous silica \\
\hline E. faecalis & Enterococcus faecalis & MRI & Magnetic resonance imaging \\
\hline ECR & Electrochemical reduction & MRSA & Methicillin-resistant Staphylococcus aureus \\
\hline EDA & Ethylenediamine & MSOT & Multispectral optoacoustic tomography \\
\hline \multirow[t]{2}{*}{ EDC } & 1-Ethyl-3-(3-dimethylamino- & $\mathrm{mT}$ & MilliTesla \\
\hline & propyl)carbodiimide & MTA & Mineral trioxide aggregate \\
\hline EDS & Energy-dispersive X-ray spectroscopy & MTX & Methotrexate \\
\hline EDTA & Ethylenediaminetetraacetic acid & MW & Molecular weight \\
\hline EG & Ethylene glycol & \multicolumn{2}{|c|}{$\left(\mathrm{NH}_{4}\right)_{10} \mathrm{~W}_{12} \mathrm{O}_{41} \cdot 5 \mathrm{H}_{2} \mathrm{O}$} \\
\hline EPR & Enhanced permeability and retention & & Ammonium tungsten oxide pentahydrate \\
\hline FA & Folic acid & $\mathrm{Na}_{2} \mathrm{MoO}_{4}$ & Sodium molybdate \\
\hline $\mathrm{Fe}$ & Iron & $\mathrm{Na}_{2} \mathrm{WO}_{4}$ & Sodium tungstate \\
\hline F-RBC & Folate modified red blood cells & $\mathrm{Na}_{3} \mathrm{PO}_{4}$ & Trisodium phosphate \\
\hline FST & Filtration-size threshold & $\mathrm{NaBH}_{4}$ & Sodium borohydride \\
\hline GA & Gambogic acid & NaI & Sodium iodide \\
\hline$g-\mathrm{C}_{3} \mathrm{~N}_{4}$ & Graphitic carbon nitride & $\mathrm{NaOH}$ & Sodium hydroxide \\
\hline
\end{tabular}




$\begin{array}{llll}\mathrm{NCs} & \text { Nanocages } & \text { Ta } & \text { Tantalum } \\ \mathrm{NH} & \text { Nanohybrid } & \text { TAA } & \text { Thioacetamide } \\ \mathrm{NH}_{4} \mathrm{H}_{2} \mathrm{PO}_{4} & \text { Ammonium dihydrogen phosphate } & \text { Tam } & \text { Trastuzumab } \\ \mathrm{NH}_{4} \mathrm{VO}_{3} & \text { Ammonium metavanadate } & \mathrm{TCID}_{50} & \text { Tissue culture infective dose 50 } \\ \mathrm{NIR} & \text { Near-infrared } & \text { TEM } & \text { Transmission electron microscope } \\ \mathrm{NO} & \text { Nitric oxide } & \text { TEMED } & \text { Tetramethylethylenediamine } \\ \mathrm{NPs} & \text { Nanoparticles } & \text { TeO } & \text { Tellurium oxide } \\ \mathrm{NRs} & \text { Nanorods } & \text { TEOS } & \text { Tetraethylorthosilicate } \\ \mathrm{NTA} & \text { Nitrilotriacetic acid } & \text { TGA } & \text { Thioglycolic acid } \\ \mathrm{NTs} & \text { Nanotubes } & \text { Th-T } & \text { Thioflavin-T } \\ \text { OA } & \text { Oleic acid } & \text { TI } & \text { Topological insulator } \\ \text { OAm } & \text { Oleylamine } & \text { TMV } & \text { Tumor microvessels } \\ \text { ONPG } & \text { ortho-Nitrophenyl- } \beta \text {-galactoside } & \text { TPGS } & \text { D- } \alpha \text {-tocopherol PEG 1000 Succinate } \\ P . \text { aeruginosa } & \text { Pseudomonas aeruginosa } & \text { US } & \text { Ultrasonography } \\ \text { PA } & \text { Photoacoustic } & \text { UV } & \text { Ultraviolet } \\ \text { PAA } & \text { Poly(acrylic acid) } & \text { VB } & \text { Valence band } \\ \text { PAH } & \text { Poly(allylamine hydrochloride) } & \text { VCAM-1 } & \text { Vascular cell adhesion molecule-1 } \\ \text { PAI } & \text { Photoacoustic imaging } & \text { W } & \text { Tungsten } \\ \text { PANI } & \text { Polyaniline } & \text { WBCs } & \text { White blood cells } \\ \text { PAT } & \text { Photoacoustic tomography } & \text { WE } & \text { Working electrode } \\ \text { PBS } & \text { Phosphate buffer saline } & \text { Yb } & \text { Ytterbium } \\ \text { PD } & \text { Polydopamine } & \text { ZP } & \text { Zinc protoporphyrin IX }\end{array}$

PDA Photodynamic antibacterials

PDT Photodynamic therapy

PEC Photoelectrochemical

PEG Poly(ethylene glycol)

PET Positron-emission tomography

PLGA Poly(lactic-co-glycolic acid)

PLL Poly-L-lysine

PM Platelet membrane

PMA Phorbol 12-myristate 13-acetate

P(NIPAM-co-AM) Poly( $\mathrm{N}$-isopropylacrylamide-co-acrylamide)

PTA

Photothermal antibacterials

PTT Photothermal therapy

PVA Poly(vinyl alcohol)

PVP Poly(vinyl pyrrolidone)

QD Quantum dots

QE Quercetin

$r_{1} \quad$ Longitudinal relaxivity

$r_{2} \quad$ Transverse relaxivity

RBCs Red blood cells

RE Reference electrode

RES Reticuloendothelial systems

RGD Arginine-glycine-aspartic acid

rGO Reduced graphene oxide

ROS Reactive oxygen species

RT Radiation therapy

S. aureus Staphylococcus aureus

S. mutans Streptococcus mutans

SCCA Squamous cell carcinoma antigen

SDBS Sodium dodecyl benzene sulfonate

SEM Scanning electron microscope

$\operatorname{SIRP} \alpha \quad$ Signal-regulated protein $\alpha$

SOD Superoxide dismutase

SPECT Single-photon emission computerized tomography

\section{Conflicts of interest}

The authors hereby declare that there are no conflicts of interest in the present study.

\section{Acknowledgements}

M.-A. Shahbazi acknowledges financial support from the Academy of Finland (grant no. 317316). P. Figueiredo acknowledges the Finnish Cultural Foundation for its financial support (decision no. 00190246). H. A. Santos acknowledges financial support from the HiLIFE Research Funds, the Sigrid Jusélius Foundation, and the Academy of Finland (grant no. 317042).

\section{References}

1 S. Tran, P.-J. DeGiovanni, B. Piel and P. Rai, Clin. Transl. Med., 2017, 6, 44.

2 G. Bor, I. D. Mat Azmi and A. Yaghmur, Ther. Delivery, 2019, 10, 113-132.

3 H. E. Karahan, C. Wiraja, C. Xu, J. Wei, Y. Wang, L. Wang, F. Liu and Y. Chen, Adv. Healthcare Mater., 2018, 7, 1701406.

4 L. Wang, C. Hu and L. Shao, Int. J. Nanomed., 2017, 12, 1227-1249.

5 S. Katsuki, T. Matoba, J. Koga, K. Nakano and K. Egashira, Front. Cardiovasc. Med., 2017, 4, 87.

6 A. Hasan, M. Morshed, A. Memic, S. Hassan, T. Webster and H. Marei, Int. J. Nanomed., 2018, 13, 5637-5655.

7 L. J. Reuter, M. A. Shahbazi, E. M. Mäkilä, J. J. Salonen, R. Saberianfar, R. Menassa, H. A. Santos, J. J. Joensuu and A. Ritala, Bioconjugate Chem., 2017, 28, 1639-1648. 
8 Y. Xiong, F. Sun, P. Liu, Z. Yang, J. Cao, H. Liu, P. Liu, J. Hu, Z. Xu and S. Yang, Chem. Eng. J., 2019, 378, 122172.

9 Y. Xiong, F. Sun, Y. Zhang, Z. Yang, P. Liu, Y. Zou, Y. Yu, F. Tong, C. Yi, S. Yang and Z. Xu, Colloids Surf., B, 2019, 173, 607-615.

10 L. Cheng, X. Wang, F. Gong, T. Liu and Z. Liu, Adv. Mater., 2019, 1902333.

11 B. Herranz-Blanco, M.-A. Shahbazi, A. R. Correia, V. Balasubramanian, T. Kohout, J. Hirvonen and H. A. Santos, Adv. Healthcare Mater., 2016, 5, 1904-1916.

12 Y. Xuan, X.-L. Song, X.-Q. Yang, R.-Y. Zhang, Z.-Y. Song, D.-H. Zhao, X.-L. Hou, J. An, X.-S. Zhang and Y.-D. Zhao, Chem. Eng. J., 2019, 375, 122000.

13 E.-K. Lim, T. Kim, S. Paik, S. Haam, Y.-M. Huh and K. Lee, Chem. Rev., 2015, 115, 327-394.

14 M. Soleymaniha, M. A. Shahbazi, A. R. Rafieerad, A. Maleki and A. Amiri, Adv. Healthcare Mater., 2019, 8, e1801137.

15 Z. Zhao, K. Xu, C. Fu, H. Liu, M. Lei, J. Bao, A. Fu, Y. Yu and W. Zhang, Biomaterials, 2019, 219, 119379.

16 L. E. Theune, J. Buchmann, S. Wedepohl, M. Molina, J. Laufer and M. Calderón, J. Controlled Release, 2019, 311-312, 147-161.

17 Y. Guo, Y. Ran, Z. Wang, J. Cheng, Y. Cao, C. Yang, F. Liu and H. Ran, Biomaterials, 2019, 219, 119370.

18 Y. Wang, J. Zhao, Z. Chen, F. Zhang, Q. Wang, W. Guo, K. Wang, H. Lin and F. Qu, Biomaterials, 2019, 217, 119282.

19 Y. Yan, K. Chang, T. Ni and K. Li, Mater. Lett., 2019, 245, 158-161.

20 Q. Zou, F. Hou, H. Wang, Y. Liao, Q. Wang and Y. Yang, Eur. Polym. J., 2019, 115, 282-289.

21 F. Wang, R. Tang, H. Yu, P. C. Gibbons and W. E. Buhro, Chem. Mater., 2008, 20, 3656-3662.

22 C. Stewart, K. Konstantinov, S. McKinnon, S. Guatelli, M. Lerch, A. Rosenfeld, M. Tehei and S. Corde, Phys. Med., 2016, 32, 1444-1452.

23 R. Zhou, H. Wang, Y. Yang, C. Zhang, X. Dong, J. Du, L. Yan, G. Zhang, Z. Gu and Y. Zhao, Biomaterials, 2019, 189, 11-22.

24 R. Hernandez-Delgadillo, C. M. García-Cuéllar, Y. SánchezPérez, N. Pineda-Aguilar, M. A. Martínez-Martínez, E. E. Rangel-Padilla, S. E. Nakagoshi-Cepeda, J. M. Solís-Soto, R. I. Sánchez-Nájera, M. A. A. Nakagoshi-Cepeda, S. Chellam and C. Cabral-Romero, Int. J. Nanomed., 2018, 13, 6089-6097.

25 H. Fan, S. Zhang, P. Ju, H. Su and S. Ai, Electrochim. Acta, 2012, 64, 171-176.

26 Y. Ramos Reynoso, A. Martinez-Ayala, M. Pal, F. ParaguayDelgado and N. R. Mathews, Adv. Powder Technol., 2018, 29, 3561-3568.

27 L. Chen, Q. Zhao and X. Ruan, Mater. Lett., 2012, 82, 112-115.

28 H. Xie, J. Shao, J. Wang, Z. Sun, X.-F. Yu and Q.-Q. Wang, RSC Adv., 2017, 7, 50234-50238.

29 M.-Q. Sun, Z.-L. Ding, H. Wang, G.-P. Yu, B.-Z. Li, M.-C. Li and M.-M. Zhen, J. Biomed. Res., 2018, 32, 380-388.

30 S. Zhang and J. Yang, Ind. Eng. Chem. Res., 2015, 54, 9913-9919.
31 A. Rajaee, X. Wensheng, L. Zhao, S. Wang, Y. Liu, Z. Wu, J. Wang and F. Si-Shen, J. Biomed. Nanotechnol., 2018, 14, 1159-1168.

32 G. Li, Y. Ding, Y. Zhang, Z. Lu, H. Sun and R. Chen, J. Colloid Interface Sci., 2011, 363, 497-503.

33 Z. Zhang, J. Wang and C. Chen, Adv. Mater., 2013, 25, 3869-3880.

34 R. Tietze, S. Lyer, S. Dürr and C. Alexiou, Nanomedicine, 2012, 7, 447-457.

35 Z.-Z. J. Lim, J.-E. J. Li, C.-T. Ng, L.-Y. L. Yung and B.-H. Bay, Acta Pharmacol. Sin., 2011, 32, 983-990.

36 Z. Li, K. Ai, Z. Yang, T. Zhang, J. Liu and X. Cui, RSC Adv., 2017, 7, 29672-29678.

37 Y. Song, J. Wang, L. Liu, Q. Sun, Q. You, Y. Cheng, Y. Wang, S. Wang, F. Tan and N. Li, Mol. Pharmaceutics, 2018, 15, 1941-1953.

38 J. Liu, X. Zheng, L. Yan, L. Zhou, G. Tian, W. Yin, L. Wang, Y. Liu, Z. Hu, Z. Gu, C. Chen and Y. Zhao, ACS Nano, 2015, 9, 696-707.

39 D. Zhou, C. Li, M. He, M. Ma, P. Li, Y. Gong, H. Ran, Z. Wang, Z. Wang, Y. Zheng and Y. Sun, J. Mater. Chem. B, 2016, 4, 4164-4181.

40 L. Sun, Q. Li, L. Zhang, H. Chai, L. Yu, Z. Xu, Y. Kang and P. Xue, Biomater. Sci., 2019, 7, 3025-3040.

41 J. C. De La Vega and U. O. Häfeli, Contrast Media Mol. Imaging, 2015, 10, 81-95.

42 B. Wei, X. Zhang, C. Zhang, Y. Jiang, Y.-Y. Fu, C. Yu, S.-K. Sun and X.-P. Yan, ACS Appl. Mater. Interfaces, 2016, 8, 12720-12726.

43 X. Yu, A. Li, C. Zhao, K. Yang, X. Chen and W. Li, ACS Nano, 2017, 11, 3990-4001.

44 S. Dadashi, R. Poursalehi and H. Delavari, Comput. Methods Biomech. Biomed. Eng. Imaging Vis., 2019, 7, 420-427.

45 M. Branca, F. Pelletier, B. Cottin, D. Ciuculescu, C.-C. Lin, R. Serra, J.-G. Mattei, M.-J. Casanove, R. Tan, M. Respaud and C. Amiens, Faraday Discuss., 2014, 175, 97-111.

46 H. Guo, X. Zhao, H. Sun, H. Zhu and H. Sun, Nanotechnology, 2019, 30, 075101.

47 G. Song, C. Liang, H. Gong, M. Li, X. Zheng, L. Cheng, K. Yang, X. Jiang and Z. Liu, Adv. Mater., 2015, 27, 6110-6117.

48 B. Wu, S.-T. Lu, H. Yu, R.-F. Liao, H. Li, B. V. Lucie Zafitatsimo, Y.-S. Li, Y. Zhang, X.-L. Zhu, H.-G. Liu, H.-B. Xu, S.-W. Huang and Z. Cheng, Biomaterials, 2018, 159, 37-47.

49 L. Sun, M. Hou, L. Zhang, D. Qian, Q. Yang, Z. Xu, Y. Kang and P. Xue, Nanomedicine, 2019, 17, 1-12.

50 R. Dou, Z. Du, T. Bao, X. Dong, X. Zheng, M. Yu, W. Yin, B. Dong, L. Yan and Z. Gu, Nanoscale, 2016, 8, 11531-11542.

51 Z. Li, Z. Li, L. Sun, B. Du, Y. Wang, G. Zhao, D. Yu, S. Yang, Y. Sun and M. Yu, Part. Part. Syst. Charact., 2018, 35, 1700337.

52 M. Ma, Y. Huang, H. Chen, X. Jia, S. Wang, Z. Wang and J. Shi, Biomaterials, 2015, 37, 447-455.

53 N. Yu, Z. Wang, J. Zhang, Z. Liu, B. Zhu, J. Yu, M. Zhu, C. Peng and Z. Chen, Biomaterials, 2018, 161, 279-291.

54 E. Li, X. Cheng, Y. Deng, J. Zhu, X. Xu, P. E. Saw, H. Gu, C. Ge and Y. Pan, Biomater. Sci., 2018, 6, 1892-1898. 
55 H. Yu, Y. Yang, T. Jiang, X. Zhang, Y. Zhao, G. Pang, Y. Feng, S. Zhang, F. Wang, Y. Wang, Y. Wang and L. W. Zhang, ACS Appl. Mater. Interfaces, 2019, 11, 27536-27547.

56 A. Muthumariyappan, U. Rajaji, S.-M. Chen, T.-W. Chen, Y.-L. Li and R. J. Ramalingam, Ultrason. Sonochem., 2019, 57, 233-241.

57 Y. Yang, R. Ouyang, L. Xu, N. Guo, W. Li, K. Feng, L. Ouyang, Z. Yang, S. Zhou and Y. Miao, J. Coord. Chem., 2015, 68, 379-397.

58 L. S. Gómez-Velázquez, A. Hernández-Gordillo, M. J. Robinson, V. J. Leppert, S. E. Rodil and M. Bizarro, Dalton Trans., 2018, 47, 12459-12467.

59 G. E. Smith, G. A. Baraff and J. M. Rowell, Phys. Rev., 1964, 135, A1118-A1124.

60 B. A. Fowler and M. J. Sexton, Handb. Toxicol. Met., 2007, 433-443.

61 J. Ni, X. Bi, Y. Jiang, L. Li and J. Lu, Nano Energy, 2017, 34, 356-366.

62 K. Ren, J. Liu, J. Liang, K. Zhang, X. Zheng, H. Luo, Y. Huang, P. Liu and X. Yu, Dalton Trans., 2013, 42, 9706-9712.

63 D. S. Bhachu, S. J. A. Moniz, S. Sathasivam, D. O. Scanlon, A. Walsh, S. M. Bawaked, M. Mokhtar, A. Y. Obaid, I. P. Parkin, J. Tang and C. J. Carmalt, Chem. Sci., 2016, 7, 4832-4841.

64 L. Zhao, X. Zhang, C. Fan, Z. Liang and P. Han, Phys. B, 2012, 407, 3364-3370.

65 Q. Zhang, D. Sando and V. Nagarajan, J. Mater. Chem. C, 2016, 4, 4092-4124.

66 N. Zhang, R. Ciriminna, M. Pagliaro and Y. J. Xu, Chem. Soc. Rev., 2014, 43, 5276-5287.

67 Y. Shimodaira, H. Kato, H. Kobayashi and A. Kudo, J. Phys. Chem. B, 2006, 110, 17790-17797.

68 Z. Zhao, Z. Li and Z. Zou, Phys. Chem. Chem. Phys., 2011, 13, 4746-4753.

69 V. D. Nithya, L. Vasylechko and R. Kalai Selvan, RSC Adv., 2014, 4, 65184-65194.

70 Y. Sun, Z. Zhao, F. Dong and W. Zhang, Phys. Chem. Chem. Phys., 2015, 17, 10383-10390.

71 F. K. Ojebuoboh, JOM, 1992, 44, 46-49.

72 C. Yang, C. Guo, W. Guo, X. Zhao, S. Liu and X. Han, ACS Appl. Nano Mater., 2018, 1, 820-830.

73 M. Drache, P. Roussel and J.-P. Wignacourt, Chem. Rev., 2007, 107, 80-96.

74 J. Eberl and H. Kisch, Photochem. Photobiol. Sci., 2008, 7, 1400-1406.

75 J. Xie, X. Lü, M. Chen, G. Zhao, Y. Song and S. Lu, Dyes Pigm., 2008, 77, 43-47.

76 F.-L. Zheng, G.-R. Li, Y.-N. Ou, Z.-L. Wang, C.-Y. Su and Y.-X. Tong, Chem. Commun., 2010, 46, 5021-5023.

77 K. Brezesinski, R. Ostermann, P. Hartmann, J. Perlich and T. Brezesinski, Chem. Mater., 2010, 22, 3079-3085.

78 L. Li and B. Yan, J. Alloys Compd., 2009, 476, 624-628.

79 L. Zhou, W. Wang, H. Xu, S. Sun and M. Shang, Chem. - Eur. J., 2009, 15, 1776-1782.

80 J. Deng and Z.-Y. Zhao, Comput. Mater. Sci., 2018, 142, 312-319.
81 K. Szostak, P. Ostaszewski, J. Pulit-Prociak and M. Banach, Pharm. Chem. J., 2019, 53, 48-51.

82 S. K. Mishra, S. Satpathy and O. Jepsen, J. Phys.: Condens. Matter, 1997, 9, 461-470.

83 P. Hu, Y. Cao, D. Jia and L. Wang, Mater. Lett., 2010, 64, 493-496.

84 M. Ramezani, A. Sobhani-Nasab and A. Davoodi, J. Mater. Sci.: Mater. Electron., 2015, 26, 5440-5445.

85 J. H. Kim, H. Park, C. H. Hsu and J. Xu, J. Phys. Chem. C, 2010, 114, 9634-9639.

86 E. B. Díaz-Cruz, O. A. Castelo-González, C. Martínez-Alonso, Z. Montiel-González, M. C. Arenas-Arrocena and H. Hu, Mater. Sci. Semicond. Process., 2018, 75, 311-318.

87 D. L. Greenaway and G. Harbeke, J. Phys. Chem. Solids, 1965, 26, 1585-1604.

88 H. Mamur, M. R. A. Bhuiyan, F. Korkmaz and M. Nil, Renewable Sustainable Energy Rev., 2018, 82, 4159-4169.

89 Y. Xu, Z. Shi, L. Zhang, E. M. B. Brown and A. Wu, Nanoscale, 2016, 8, 12715-12722.

90 K.-L. Zhang, C.-M. Liu, F.-Q. Huang, C. Zheng and W.-D. Wang, Appl. Catal., B, 2006, 68, 125-129.

91 G. Catalan and J. F. Scott, Adv. Mater., 2009, 21, 2463-2485.

92 P. S. V. Mocherla, C. Karthik, R. Ubic, M. S. Ramachandra Rao and C. Sudakar, Appl. Phys. Lett., 2013, 103, 022910.

93 R. Moubah, G. Schmerber, O. Rousseau, D. Colson and M. Viret, Appl. Phys. Express, 2012, 5, 3.

94 R. W. Wolfe, R. E. Newnahm and M. I. Kay, Solid State Commun., 1969, 7, 1797-1801.

95 T. Saison, P. Gras, N. Chemin, C. Chanéac, O. Durupthy, V. Brezová, C. Colbeau-Justin and J. P. Jolivet, J. Phys. Chem. C, 2013, 117, 22656-22666.

96 B. Y. Alfaifi, A. A. Tahir and K. G. U. Wijayantha, Sol. Energy Mater. Sol. Cells, 2019, 195, 134-141.

97 C. Zhang, J. Ren, J. Hua, L. Xia, J. He, D. Huo and Y. Hu, ACS Appl. Mater. Interfaces, 2018, 10, 1132-1146.

98 B. Senthilkumar, R. K. Selvan, L. Vasylechko and M. Minakshi, Solid State Sci., 2014, 35, 18-27.

99 Q. He, Y. Ni and S. Ye, RSC Adv., 2017, 7, 27089-27099.

100 T. Zhang, J. Huang, S. Zhou, H. Ouyang, L. Cao and A. Li, Ceram. Int., 2013, 39, 7391-7394.

101 T. Saison, N. Chemin, C. Chanéac, O. Durupthy, L. Mariey, F. Maugé, V. Brezová and J. P. Jolivet, J. Phys. Chem. C, 2015, 119, 12967-12977.

102 F. W. P. Ribeiro, M. F. Gromboni, F. Marken and L. H. Mascaro, Int. J. Hydrogen Energy, 2016, 41, 17380-17389.

103 A. Malathi, J. Madhavan, M. Ashokkumar and P. Arunachalam, Appl. Catal., A, 2018, 555, 47-74.

104 J. Wang, J. Li, H. Li, S. Duan, S. Meng, X. Fu and S. Chen, Chem. Eng. J., 2017, 330, 433-441.

105 P. Arunkumar, C. Jayajothi, D. Jeyakumar and N. Lakshminarasimhan, RSC Adv., 2012, 2, 1477-1485.

106 X. Zhou and X. Wang, Luminescence, 2013, 28, 685-689.

107 M. Yang, N. K. Shrestha, R. Hahn and P. Schmuki, Electrochem. Solid-State Lett., 2010, 13, C5-C8.

108 R. Chen, M. H. So, J. Yang, F. Deng, C. M. Che and H. Sun, Chem. Commun., 2006, 2265-2267. 
109 F. Dong, W. K. Ho, S. C. Lee, Z. Wu, M. Fu, S. Zou and Y. Huang, J. Mater. Chem., 2011, 21, 12428-12436.

110 M. A. Martínez-Martínez, R. Hernandez-Delgadillo, B. S. Abada, N. Pineda-Aguilar, J. M. Solís-Soto, M. A. A. Nakagoshi-Cepeda, S. E. Nakagoshi-Cepeda, S. Chellam, R. I. Sánchez-Nájera and C. Cabral-Romero, Dent. Mater. J., 2019, 38, 611-620.

111 Y. Tian and J. Toudert, J. Nanotechnol., 2018, 3250932.

112 C. Chen, J. Cheng, S. Yu, L. Che and Z. Meng, J. Cryst. Growth, 2006, 291, 135-139.

113 J. Wu, F. Qin, Z. Lu, H. J. Yang and R. Chen, Nanoscale Res. Lett., 2011, 6, 66.

114 A. Manzoor, A. M. Afzal, M. Umair, A. Ali, M. Rizwan and M. Z. Yaqoob, J. Magn. Magn. Mater., 2015, 393, 269-272.

115 H. Wang, J. J. Zhu, J. M. Zhu and H. Y. Chen, J. Phys. Chem. $B, 2002,106,3848-3854$.

116 F. Majid and S. T. Mirza, Mater. Today: Proc., 2015, 2, 5293-5297.

117 A. Purkayastha, S. Kim, D. D. Gandhi, P. G. Ganesan, T. Borca-Tasciuc and G. Ramanath, Adv. Mater., 2006, 18, 2958-2963.

118 O. V. Kharissova, M. Osorio, M. Garza and B. I. Kharisov, Synth. React. Inorg., Met.-Org., Nano-Met. Chem., 2008, 38, 567-572.

119 M. Assis, E. Cordoncillo, R. Torres-Mendieta, H. BeltránMir, G. Mínguez-Vega, A. F. Gouveia, E. Leite, J. Andrés and E. Longo, Phys. Chem. Chem. Phys., 2018, 20, 13693-13696.

120 A. L. Brown and A. M. Goforth, Chem. Mater., 2012, 24, 1599-1605.

121 A. L. Brown, P. C. Naha, V. Benavides-Montes, H. I. Litt, A. M. Goforth and D. P. Cormode, Chem. Mater., 2014, 26, 2266-2274.

122 J. S. Son, K. Park, M.-K. Han, C. Kang, S.-G. Park, J.-H. Kim, W. Kim, S.-J. Kim and T. Hyeon, Angew. Chem., Int. Ed., 2011, 50, 1363-1366.

123 E. H. Bindewald, A. F. Schibelbain, M. A. P. Papi, E. G. C. Neiva, A. J. G. Zarbin, M. F. Bergamini and L. H. MarcolinoJúnior, Mater. Sci. Eng., C, 2017, 79, 262-269.

124 L. Torrisi, L. Silipigni, N. Restuccia, S. Cuzzocrea, M. Cutroneo, F. Barreca, B. Fazio, G. Di Marco and S. Guglielmino, J. Phys. Chem. Solids, 2018, 119, 62-70.

125 Z. A. Zulkifli, K. A. Razak, W. N. W. A. Rahman and S. Z. Abidin, Journal of Physics: Conference Series, Institute of Physics Publishing, 2018, vol. 1082.

126 M. Anilkumar, R. Pasricha and V. Ravi, Ceram. Int., 2005, 31, 889-891.

127 M. Mallahi, A. Shokuhfar, M. R. Vaezi, A. Esmaeilirad and V. Mazinani, Am. J. Eng. Res., 2014, 3, 162-165.

128 W. Li, Mater. Chem. Phys., 2006, 99, 174-180.

129 Q. Huang, S. Zhang, C. Cai and B. Zhou, Mater. Lett., 2011, 65, 988-990.

130 Z. Ali, C. Cao, J. Li, Y. Wang, T. Cao, M. Tanveer, M. Tahir, F. Idrees and F. K. Butt, J. Power Sources, 2013, 229, 216-222.

131 Y. Jiang, Y. J. Zhu and G. F. Cheng, Cryst. Growth Des., 2006, 6, 2174-2176.
132 X. Qiu, C. Burda, R. Fu, L. Pu, H. Chen and J. Zhu, J. Am. Chem. Soc., 2004, 126, 16276-16277.

133 Y. Sang, G. Dai, L. Wang, X. Gao and C. Fang, ChemistrySelect, 2018, 3, 7123-7128.

134 Q. Wang, X. Wang, W. Lou and J. Hao, New J. Chem., 2010, 34, 1930-1935.

135 J. Lu, Q. Han, X. Yang, L. Lu and X. Wang, Mater. Lett., 2007, 61, 2883-2886.

136 G. Xing, Z. Feng, G. Chen, W. Yao and X. Song, Mater. Lett., 2003, 57, 4555-4559.

137 P. Dharmaiah, C. H. Lee, B. Madavali and S. J. Hong, Arch. Metall. Mater., 2017, 62, 1005-1010.

138 Y. Xu, Z. Ren, G. Cao, W. Ren, K. Deng and Y. Zhong, Phys. $B, 2009$, 404, 4029-4033.

139 Z. Li, R. Teng, S. Zheng, Y. Zhang, T. Huang and G. Lu, J. Cryst. Growth, 2014, 406, 104-110.

140 N. Watanabe, J. Kawamata and N. Toshima, Chem. Lett., 2004, 33, 1368-1369.

141 H. Deng, J. Wang, Q. Peng, X. Wang and Y. Li, Chem. - Eur. J., 2005, 11, 6519-6524.

142 J. Ma, X. Liu, J. Lian, X. Duan and W. Zheng, Cryst. Growth Des., 2010, 10, 2522-2527.

143 S. Wu, C. Wang, Y. Cui, W. Hao, T. Wang and P. Brault, Mater. Lett., 2011, 65, 1344-1347.

144 L. Song, S. Zhang and Q. Wei, Ind. Eng. Chem. Res., 2012, 51, 1193-1197.

145 Y. Wang, G. Xu, Z. Ren, X. Wei, W. Weng, P. Du, G. Shen and G. Han, Ceram. Int., 2008, 34, 1569-1571.

146 S. Ghosh, S. Dasgupta, A. Sen and H. S. Maiti, J. Am. Ceram. Soc., 2005, 88, 1349-1352.

147 S. Sharma, P. Saravanan, O. P. Pandey, V. T. P. Vinod, M. Černík and P. Sharma, J. Magn. Magn. Mater., 2016, 401, 180-187.

148 M. Hasan, M. F. Islam, R. Mahbub, M. S. Hossain and M. A. Hakim, Mater. Res. Bull., 2016, 73, 179-186.

149 T. Soltani and M. H. Entezari, Chem. Eng. J., 2013, 223, 145-154.

150 Z. Cui, H. Yang, B. Wang, R. Li and X. Wang, Nanoscale Res. Lett., 2016, 11, 190.

151 L. Wu, J. Bi, Z. Li, X. Wang and X. Fu, Catal. Today, 2008, 131, 15-20.

152 V. D. Nithya, R. K. Selvan, D. Kalpan, L. Vasylechko and C. Sanjeeviraj, Electrochim. Acta, 2013, 109, 720-731.

153 A. Phuruangrat, S. Putdum, P. Dumrongrojthanath, S. Thongtem and T. Thongtem, J. Nanomater., 2015, 135735.

154 J. Yesuraj and S. A. Suthanthiraraj, J. Mater. Sci.: Mater. Electron., 2018, 29, 5862-5872.

155 L. Zhang, D. Chen and X. Jiao, J. Phys. Chem. B, 2006, 110, 2668-2673.

156 W. Liu, X. Wang, L. Cao, G. Su, L. Zhang and Y. Wang, Sci. China: Chem., 2011, 54, 724-729.

157 M. Shang, W. Wang, L. Zhou, S. Sun and W. Yin, J. Hazard. Mater., 2009, 172, 338-344.

158 Y. Wang, X. Guan, L. Li and G. Li, CrystEngComm, 2012, 14, 7907-7914. 
159 G. Liu, S. Liu, Q. Lu, H. Sun and Z. Xiu, Ind. Eng. Chem. Res., 2014, 53, 13023-13029.

160 J. Geng, W. H. Hou, Y. N. Lv, J. J. Zhu and H. Y. Chen, Inorg. Chem., 2005, 44, 8503-8509.

161 H. Y. Liang, Y. X. Yang, J. C. Tang and M. Ge, Mater. Sci. Semicond. Process., 2013, 16, 1650-1654.

162 R. Chen, G. Cheng, M. H. So, J. Wu, Z. Lu, C.-M. Che and H. Sun, Mater. Res. Bull., 2010, 45, 654-658.

163 A. R. Badireddy, R. Hernandez-Delgadillo, R. I. SánchezNájera, S. Chellam and C. Cabral-Romero, J. Nanopart. Res., 2014, 16, 2456.

164 H. Hayashi and Y. Hakuta, Materials, 2010, 3, 3794-3817.

165 J. Li, Q. Wu and J. Wu, Handbook of Nanoparticles, Springer International Publishing, Cham, 2015, pp. 1-28.

166 G. Biasotto, A. Z. Simões, C. R. Foschini, M. A. Zaghete, J. A. Varela and E. Longo, Mater. Res. Bull., 2011, 46, 2543-2547.

167 E.-W. Shi, C.-T. Xia, W.-Z. Zhong, B.-G. Wang and C.-D. Feng, J. Am. Ceram. Soc., 1997, 80, 1567-1572.

168 C. N. R. Rao and K. Biswas, Essentials of Inorganic Materials Synthesis, John Wiley \& Sons, Inc, 2015, pp. 57-95.

169 M. Antonietti, D. Kuang, B. Smarsly and Y. Zhou, Angew. Chem., Int. Ed., 2004, 43, 4988-4992.

170 S. E. Shirsath, S. S. Jadhav, M. L. Mane and S. Li, Handbook of Sol-Gel Science and Technology, Springer International Publishing, Cham, 2016, pp. 1-41.

171 J.-H. Xu, H. Ke, D.-C. Jia, W. Wang and Y. Zhou, J. Alloys Compd., 2009, 472, 473-477.

172 G. J. Owens, R. K. Singh, F. Foroutan, M. Alqaysi, C.-M. Han, C. Mahapatra, H.-W. Kim and J. C. Knowles, Prog. Mater. Sci., 2016, 77, 1-79.

173 S. Esposito, Materials, 2019, 12, 668.

174 T. Gao, Z. Chen, Y. Zhu, F. Niu, Q. Huang, L. Qin, X. Sun and Y. Huang, Mater. Res. Bull., 2014, 59, 6-12.

175 X. Wang, Y. Zhang and Z. Wu, Mater. Lett., 2010, 64, 486-488.

176 R. Uppuluri, A. Sen Gupta, A. S. Rosas and T. E. Mallouk, Chem. Soc. Rev., 2018, 47, 2401-2430.

177 W. Wang, N. Li, Y. Chi, Y. Li, W. Yan, X. Li and C. Shao, Ceram. Int., 2013, 39, 3511-3518.

178 M. A. Malik, M. Y. Wani and M. A. Hashim, Arab. J. Chem., 2012, 5, 397-417.

179 N. Das, R. Majumdar, A. Sen and H. S. Maiti, Mater. Lett., 2007, 61, 2100-2104.

180 Y. Chen, X. Xu, J. Fang, G. Zhou, Z. Liu, S. Wu, W. Xu, J. Chu and X. Zhu, Sci. World J., 2014, 647040.

181 W. Z. Wang, B. Poudel, Y. Ma and Z. F. Ren, J. Phys. Chem. $B, 2006,110,25702-25706$.

182 B. Qian, X. Li and Z. Song, J. Am. Ceram. Soc., 2018, 101, 4425-4429.

183 D. H. Webber and R. L. Brutchey, Inorg. Chem., 2011, 50, 723-725.

184 F. Fiévet, S. Ammar-Merah, R. Brayner, F. Chau, M. Giraud, F. Mammeri, J. Peron, J. Y. Piquemal, L. Sicard and G. Viau, Chem. Soc. Rev., 2018, 47, 5187-5233.

185 G. R. Li, H. Xu, X. F. Lu, J. X. Feng, Y. X. Tong and C. Y. Su, Nanoscale, 2013, 5, 4056-4069.
186 S. Li, M. S. Toprak, H. M. A. Soliman, J. Zhou, M. Muhammed, D. Platzek and E. Müller, Chem. Mater., 2006, 18, 3627-3633.

187 F. L. Zheng, G. R. Li, Y. N. Ou, Z. L. Wang, C. Y. Su and Y. X. Tong, Chem. Commun., 2010, 46, 5021-5023.

188 W. B. Zhao, J. J. Zhu, J. Z. Xu and H. Y. Chen, Inorg. Chem. Commun., 2004, 7, 847-850.

189 S. Pradhan, R. Das, R. Bhar, R. Bandyopadhyay and P. Pramanik, J. Nanopart. Res., 2017, 19, 69.

190 X. H. Liao, H. Wang, J. J. Zhu and H. Y. Chen, Mater. Res. Bull., 2001, 36, 2339-2346.

191 J. H. Bang and K. S. Suslick, Adv. Mater., 2010, 22, 1039-1059.

192 H. Xu, B. W. Zeiger and K. S. Suslick, Chem. Soc. Rev., 2013, 42, 2555-2567.

193 K. S. Suslick and D. J. Flannigan, Annu. Rev. Phys. Chem., 2008, 59, 659-683.

194 L. Zhang, W. Wang, J. Yang, Z. Chen, W. Zhang, L. Zhou and S. Liu, Appl. Catal., A, 2006, 308, 105-110.

195 B. Kumar, G. Kaur, R. K. Verma, A. Bahadur and S. B. Rai, RSC Adv., 2016, 6, 26984-26992.

196 C. Khatua, D. Bhattacharya, B. Kundu, V. K. Balla, S. Bodhak and S. Goswami, Adv. Eng. Mater., 2018, 20, 1800329.

197 M. Suresh and A. Sivasamy, J. Environ. Chem. Eng., 2018, 6, 3745-3756.

198 O. Rabin, J. Manuel Perez, J. Grimm, G. Wojtkiewicz and R. Weissleder, Nat. Mater., 2006, 5, 118-122.

199 Y. Wei, H. Zhou, H. Zhang, X. Zhu, G. Liu, Y. Li and W. Cai, ChemPhysChem, 2017, 18, 1146-1154.

200 R. Tao, C. Shao, X. Li, X. Li, S. Liu, S. Yang, C. Zhao and Y. Liu, J. Colloid Interface Sci., 2018, 529, 404-414.

201 J. Su, L. Guo, N. Bao and C. A. Grimes, Nano Lett., 2011, 11, 1928-1933.

202 L. Sun, L. Xiang, X. Zhao, C.-J. Jia, J. Yang, Z. Jin, X. Cheng and W. Fan, ACS Catal., 2015, 5, 3540-3551.

203 K. Wang, C. Shao, X. Li, F. Miao, N. Lu and Y. Liu, Materials, 2016, 9, 1-12.

204 X. Chen, Y. Lou, S. Dayal, X. Qiu, R. Krolicki, C. Burda, C. Zhao and J. Becker, J. Nanosci. Nanotechnol., 2005, 5, 1408-1420.

205 S. Xue, H. He, Q. Fan, C. Yu, K. Yang, W. Huang, Y. Zhou and Y. Xie, J. Environ. Sci., 2017, 60, 70-77.

206 H. Li, W. Li, X. Liu, C. Ren, X. Miao and X. Li, Appl. Surf. Sci., 2019, 463, 556-565.

207 G. S. Lotey and N. K. Verma, Superlattices Microstruct., 2013, 53, 184-194.

208 S.-T. Lu, D. Xu, R.-F. Liao, J.-Z. Luo, Y.-H. Liu, Z.-H. Qi, C.-J. Zhang, N.-L. Ye, B. Wu and H.-B. Xu, Comput. Struct. Biotechnol. J., 2019, 17, 619-627.

209 M. Yao, M. Ma, Y. Chen, X. Jia, G. Xu, H. Xu, H. Chen and R. Wu, Biomaterials, 2014, 35, 8197-8205.

210 J. M. Kinsella, R. E. Jimenez, P. P. Karmali, A. M. Rush, V. R. Kotamraju, N. C. Gianneschi, E. Ruoslahti, D. Stupack and M. J. Sailor, Angew. Chem., Int. Ed., 2011, 50, 12308-12311.

211 K. Wang, J. Zhuang, Y. Liu, M. Xu, J. Zhuang, Z. Chen, Y. Wei and Y. Zhang, Carbohydr. Polym., 2018, 184, 445-452. 
212 E. R. Swy, A. S. Schwartz-Duval, D. D. Shuboni, M. T. Latourette, C. L. Mallet, M. Parys, D. P. Cormode and E. M. Shapiro, Nanoscale, 2014, 6, 13104-13112.

213 Z. Li, J. Liu, Y. Hu, Z. Li, X. Fan, Y. Sun, F. Besenbacher, C. Chen and M. Yu, Biomaterials, 2017, 141, 284-295.

214 F. Du, J. Lou, R. Jiang, Z. Fang, X. Zhao, Y. Niu, S. Zou, M. Zhang, A. Gong and C. Wu, Int. J. Nanomed., 2017, 12, 5973-5992.

215 Y. Zhao, L. Detering, D. Sultan, M. L. Cooper, M. You, S. Cho, S. L. Meier, H. Luehmann, G. Sun, M. Rettig, F. Dehdashti, K. L. Wooley, J. F. DiPersio and Y. Liu, ACS Nano, 2016, 10, 5959-5970.

216 Q. Liu, M. Chen, Y. Sun, G. Chen, T. Yang, Y. Gao, X. Zhang and F. Li, Biomaterials, 2011, 32, 8243-8253.

217 H. L. Dupont, Drug Intell. Clin. Pharm., 1987, 21, 687-693.

218 D. Y. Graham and S.-Y. Lee, Clin. Gastroenterol., 2015, 44, 537-563.

219 S. L. Gorbach, Gastroenterology, 1990, 99, 863-875.

220 S. Wagner, M. Gebel, K. Haruma, W. Bar, P. Lange, J. Freise, U. Gladziwa and F. W. Schmidt, Gut, 1992, 33, 179-183.

221 V. Mishra, V. Baranwal, R. K. Mishra, S. Sharma, B. Paul and A. C. Pandey, Sci. Rep., 2017, 7, 18032.

222 K. Ai, Y. Liu, J. Liu, Q. Yuan, Y. He and L. Lu, Adv. Mater., 2011, 23, 4886-4891.

223 M. Esquivel-Gaon, S. Anguissola, D. Garry, A. del, C. Gallegos-Melgar, J. M. Saldaña, K. A. Dawson, A. De Vizcaya-Ruiz and L. M. Del Razo, RSC Adv., 2015, 5, 27295-27304.

224 Y. Luo, C. Wang, Y. Qiao, M. Hossain, L. Ma and M. Su, J. Mater. Sci.: Mater. Med., 2012, 23, 2563-2573.

225 J. S. Suk, Q. Xu, N. Kim, J. Hanes and L. M. Ensign, Adv. Drug Delivery Rev., 2016, 99, 28-51.

226 P. Grenier, I. M. de, O. Viana, E. M. Lima and N. Bertrand, J. Controlled Release, 2018, 287, 121-131.

227 K. Shiraishi, K. Kawano, Y. Maitani, T. Aoshi, K. J. Ishii, Y. Sanada, S. Mochizuki, K. Sakurai and M. Yokoyama, J. Controlled Release, 2016, 234, 59-67.

228 Q. Xia, Y. Zhang, Z. Li, X. Hou and N. Feng, Acta Pharm. Sin. B, 2019, 9, 675-689.

229 J. Deng, S. Xu, W. Hu, X. Xun, L. Zheng and M. Su, Biomaterials, 2018, 154, 24-33.

230 N. Hoshyar, S. Gray, H. Han and G. Bao, Nanomedicine, 2016, 11, 673-692.

231 Q. Feng, Y. Liu, J. Huang, K. Chen, J. Huang and K. Xiao, Sci. Rep., 2018, 8, 2082.

232 Y. Chen, G. Zhao, S. Wang, Y. He, S. Han, C. Du, S. Li, Z. Fan, C. Wang and J. Wang, Biomater. Sci., 2019, 7, 3450-3459.

233 D. Buergy, F. Wenz, C. Groden and M. A. Brockmann, Int. J. Cancer, 2012, 130, 2747-2760.

234 L. Borsig, R. Wong, J. Feramisco, D. R. Nadeau, N. M. Varki and A. Varki, Proc. Natl. Acad. Sci. U. S. A., 2001, 98, 3352-3357.

235 J. P. Stone and D. D. Wagner, J. Clin. Invest., 1993, 92, 804-813.
236 Z. Li, Y. Hu, M. Chang, K. A. Howard, X. Fan, Y. Sun, F. Besenbacher and M. Yu, Nanoscale, 2016, 8, 16005-16016.

237 L. Dong, P. Zhang, X. Liu, R. Deng, K. Du, J. Feng and H. Zhang, ACS Appl. Mater. Interfaces, 2019, 11, 7774-7781.

238 ASTM F756-17, Standard Practice for Assessment of Hemolytic Properties of Materials, West Conshohocken, PA, 2017.

239 X. Zheng, J. Shi, Y. Bu, G. Tian, X. Zhang, W. Yin, B. Gao, Z. Yang, Z. Hu, X. Liu, L. Yan, Z. Gu and Y. Zhao, Nanoscale, 2015, 7, 12581-12591.

240 N. He, X. Li, D. Feng, M. Wu, R. Chen, T. Chen, D. Chen and X. Feng, Chem. Res. Toxicol., 2013, 26, 89-95.

241 S. E. Lepage and A. E. E. Bruce, Int. J. Dev. Biol., 2010, 54, 1213-1228.

242 M. Tada and M. Kai, Zebrafish, 2009, 6, 29-40.

243 C. J. Weijer, J. Cell Sci., 2009, 122, 3215-3223.

244 H. Aviv, S. Bartling, I. Grinberg and S. Margel, J. Biomed. Mater. Res., 2013, 101B, 131-138.

245 X.-D. Zhang, J. Chen, Y. Min, G. B. Park, X. Shen, S.-S. Song, Y.-M. Sun, H. Wang, W. Long, J. Xie, K. Gao, L. Zhang, S. Fan, F. Fan and U. Jeong, Adv. Funct. Mater., 2014, 24, 1718-1729.

246 X.-D. Zhang, D. Wu, X. Shen, P.-X. Liu, N. Sun, B. Zhang, H. Fan, Y.-M. Sun, L.-A. Zhang and F.-Y. Fan, Int. J. Nanomed., 2011, 6, 2071.

247 X.-D. Zhang, D. Wu, X. Shen, P.-X. Liu, F.-Y. Fan and S.-J. Fan, Biomaterials, 2012, 33, 4628-4638.

248 L. Gu, R. H. Fang, M. J. Sailor and J.-H. Park, ACS Nano, 2012, 6, 4947-4954.

249 X.-D. Zhang, Y. Jing, S. Song, J. Yang, J.-Y. Wang, X. Xue, Y. Min, G. Park, X. Shen, Y.-M. Sun and U. Jeong, Nanomedicine, 2017, 13, 1597-1605.

250 J. Du, Z. Gu, L. Yan, Y. Yong, X. Yi, X. Zhang, J. Liu, R. Wu, C. Ge, C. Chen and Y. Zhao, Adv. Mater., 2017, 29, 1701268.

251 H. Soo Choi, W. Liu, P. Misra, E. Tanaka, J. P. Zimmer, B. Itty Ipe, M. G. Bawendi and J. V. Frangioni, Nat. Biotechnol., 2007, 25, 1165-1170.

252 M. Yu and J. Zheng, ACS Nano, 2015, 9, 6655-6674.

253 P. Lei, P. Zhang, Q. Yuan, Z. Wang, L. Dong, S. Song, X. Xu, X. Liu, J. Feng and H. Zhang, ACS Appl. Mater. Interfaces, 2015, 7, 26346-26354.

254 L. Cheng, S. Shen, S. Shi, Y. Yi, X. Wang, G. Song, K. Yang, G. Liu, T. E. Barnhart, W. Cai and Z. Liu, Adv. Funct. Mater., 2016, 26, 2185-2197.

255 S.-M. Zhou, D.-K. Ma, S.-H. Zhang, W. Wang, W. Chen, S.-M. Huang and K. Yu, Nanoscale, 2016, 8, 1374-1382.

256 Z. Li, J. Liu, Y. Hu, K. A. Howard, Z. Li, X. Fan, M. Chang, Y. Sun, F. Besenbacher, C. Chen and M. Yu, ACS Nano, 2016, 10, 9646-9658.

257 Y. Wang, Y. Wu, Y. Liu, J. Shen, L. Lv, L. Li, L. Yang, J. Zeng, Y. Wang, L. W. Zhang, Z. Li, M. Gao and Z. Chai, Adv. Funct. Mater., 2016, 26, 5335-5344.

258 P. Lei, R. An, X. Zhai, S. Yao, L. Dong, X. Xu, K. Du, M. Zhang, J. Feng and H. Zhang, J. Mater. Chem. C, 2017, 5, 9659-9665.

259 J. Liu, P. Wang, X. Zhang, L. Wang, D. Wang, Z. Gu, J. Tang, M. Guo, M. Cao, H. Zhou, Y. Liu and C. Chen, ACS Nano, 2016, 10, 4587-4598. 
260 P. C. Naha, A. Al Zaki, E. Hecht, M. Chorny, P. Chhour, E. Blankemeyer, D. M. Yates, W. R. T. Witschey, H. I. Litt, A. Tsourkas and D. P. Cormode, J. Mater. Chem. B, 2014, 2, 8239-8248.

261 X. Hu, J. Sun, F. Li, R. Li, J. Wu, J. He, N. Wang, J. Liu, S. Wang, F. Zhou, X. Sun, D. Kim, T. Hyeon and D. Ling, Nano Lett., 2018, 18, 1196-1204.

262 Y. N. Slavin, J. Asnis, U. O. Häfeli and H. Bach, J. Nanobiotechnol., 2017, 15, 65.

263 P. Singh, A. Garg, S. Pandit, V. R. S. S. Mokkapati and I. Mijakovic, Nanomater, 2018, 8, 1009.

264 M.-A. Shahbazi, M. P. Ferreira and H. A. Santos, Nanomedicine, 2019, 14, 2269-2272.

265 A. Gupta, S. Mumtaz, C.-H. Li, I. Hussain and V. M. Rotello, Chem. Soc. Rev., 2019, 48, 415-427.

266 R. Y. Pelgrift and A. J. Friedman, Adv. Drug Delivery Rev., 2013, 65, 1803-1815.

267 P. K. Stoimenov, R. L. Klinger, G. L. Marchin and K. J. Klabunde, Langmuir, 2002, 18, 6679-6686.

268 J. A. Lemire, J. J. Harrison and R. J. Turner, Nat. Rev. Microbiol., 2013, 11, 371-384.

269 S. Shrivastava, T. Bera, A. Roy, G. Singh, P. Ramachandrarao and D. Dash, Nanotechnology, 2007, 18, 225103.

270 R. Mohan, Nat. Chem., 2010, 2, 336.

271 M. D. Manhart, Rev. Infect. Dis., 1990, 12, S11-S15.

272 L. F. Dalvand, F. Hosseini, S. M. Dehaghi and E. S. Torbati, Iran. J. Biotechnol., 2018, 16, e2102.

273 C. Cabral-Romero, D. Hernandez-Delgadillo, D. VelascoArias, K. Diaz, C. Cabral-Romero, M. A. Niño-Arevalo and C. De la Garza-Ramos, Int. J. Nanomed., 2012, 7, 2109.

274 C. Cabral-Romero, R. Hernandez-Delgadillo, J. J. VelascoArias, D. Martinez-Sanmiguel, D. Diaz, K. Zumeta-Dubé and C. Niño-Arevalo, Int. J. Nanomed., 2013, 8, 1645.

275 A. R. Badireddy, S. M. Marinakos, S. Chellam and M. R. Wiesner, Surf. Innov., 2013, 1, 181-189.

276 P. Nazari, R. Dowlatabadi-Bazaz, M. R. Mofid, M. R. Pourmand, N. E. Daryani, M. A. Faramarzi, Z. Sepehrizadeh and A. R. Shahverdi, Appl. Biochem. Biotechnol., 2014, 172, 570-579.

277 Y. Luo, M. Hossain, C. Wang, Y. Qiao, J. An, L. Ma and M. Su, Nanoscale, 2013, 5, 687-694.

278 A. L. Vega-Jiménez, A. Almaguer-Flores, M. Flores-Castañeda, E. Camps, M. Uribe-Ramírez, O. G. Aztatzi-Aguilar and A. De Vizcaya-Ruiz, Nanotechnology, 2017, 28, 435101.

279 V. Campos, A. Almaguer-Flores, D. Velasco-Aria, D. Díaz and S. E. Rodil, J. Mater. Sci. Eng. A, 2018, 8, 142-146.

280 M. Varposhti, A. Abdi Ali and P. Mohammadi, Jundishapur J. Microbiol., 2014, 7, e9142.

281 X. Long, Q. Chen, L. Yu, X. Liang, W. Liu and H. Lu, Helicobacter, 2018, 23, e12485.

282 P. Domenico, R. J. Salo, S. G. Novick, P. E. Schoch, K. Van Horn and B. A. Cunha, Antimicrob. Agents Chemother., 1997, 41, 1697-1703.

283 J. P. Folsom, B. Baker and P. S. Stewart, J. Appl. Microbiol., 2011, 111, 989-996.

284 R. Hernandez-Delgadillo, C. Del Angel-Mosqueda, J. M. Solís-Soto, S. Munguia-Moreno, N. Pineda-Aguilar,
R. I. Sánchez-Nájera, S. Chellam and C. Cabral-Romero, Dent. Mater. J., 2017, 36, 503-510.

285 G. Ciobanu, A. M. Bargan and C. Luca, JOM, 2015, 67, 2534-2542.

286 M. Shakibaie, E. Hajighasemi, M. Adeli-Sardou, M. Doostmohammadi and $\mathrm{H}$. Forootanfar, IET Nanobiotechnol., 2019, 13, 377-381.

287 Z. Tarjoman, S. M. Ganji and S. Mehrabian, Merit Research Journal of Medicine and Medical Sciences, 2015, 3, 387-393.

288 R. Sharma, Uma, S. Singh, A. Verma and M. Khanuja, J. Photochem. Photobiol., B, 2016, 162, 266-272.

289 R. Wang, B. Zhang, Z. Liang, Y. He, Z. Wang, X. Ma, X. Yao, J. Sun and J. Wang, Appl. Catal., B, 2019, 241, 167-177.

290 C.-L. Hsu, Y.-J. Li, H.-J. Jian, S. G. Harroun, S.-C. Wei, R. Ravindranath, J.-Y. Lai, C.-C. Huang and H.-T. Chang, Nanoscale, 2018, 10, 11808-11819.

291 J. Li, Y. Yu and L. Zhang, Nanoscale, 2014, 6, 8473-8488.

292 H. Sun, Z. Jiang, D. Wu, L. Ye, T. Wang, B. Wang, T. An and P. K. Wong, ChemSusChem, 2019, 12, 890-897.

293 G. Gorle, A. Bathinapatla, Y.-Z. Chen and Y.-C. Ling, RSC Adv., 2018, 8, 19827-19834.

294 Y. Li, X. Liu, L. Tan, Z. Cui, D. Jing, X. Yang, Y. Liang, Z. Li, S. Zhu, Y. Zheng, K. W. K. Yeung, D. Zheng, X. Wang and S. Wu, Adv. Funct. Mater., 2019, 29, 1900946.

295 J. Ouyang, M. Wen, W. Chen, Y. Tan, Z. Liu, Q. Xu, K. Zeng, L. Deng and Y.-N. Liu, Chem. Commun., 2019, 55, 4877-4880.

296 M. V. Werrett, M. E. Herdman, R. Brammananth, U. Garusinghe, W. Batchelor, P. K. Crellin, R. L. Coppel and P. C. Andrews, Chem. - Eur. J., 2018, 24, 12938-12949.

297 A. R. Indurkar, V. D. Sangoi, P. B. Patil and M. S. Nimbalkar, IET Nanobiotechnol., 2018, 12, 496-499.

298 M. Gosau, M. Haupt, S. Thude, M. Strowitzki, B. Schminke and R. Buergers, J. Biomed. Mater. Res., Part B, 2016, 104, 1571-1579.

299 J. An, A. Sun, Y. Qiao, P. Zhang and M. Su, J. Mater. Sci.: Mater. Med., 2015, 26, 68.

300 D.-J. Lin, M.-T. Tsai, T.-M. Shieh, H.-L. Huang, J.-T. Hsu, Y.-C. Ko and L.-J. Fuh, J. Biomater. Appl., 2013, 27, 553-563.

301 M. Collinsova and J. Jiracek, Curr. Med. Chem., 2000, 7, 629-647.

302 B. E. Nagay, C. Dini, J. M. Cordeiro, A. P. Ricomini-Filho, E. D. de Avila, E. C. Rangel, N. C. da Cruz and V. A. R. Barão, ACS Appl. Mater. Interfaces, 2019, 11, 18186-18202. 303 E. Ulkür, O. Oncül, H. Karagöz, B. Celiköz and S. Cavuşlu, J. Burn Care Rehabil., 2005, 26, 430-433.

304 E. V. Capparelli, R. Bricker-Ford, M. J. Rogers, J. H. McKerrow and S. L. Reed, Antimicrob. Agents Chemother., 2017, 61, e01947-16.

305 P. Figueiredo, V. Balasubramanian, M. A. Shahbazi, A. Correia, D. Wu, C. G. Palivan, J. T. Hirvonen and H. A. Santos, Int. J. Pharm., 2016, 511, 794-803.

306 L. F. Fraceto, E. V. R. Campos, S. Habtemariam, M. del P. Rodriguez-Torres, J. K. Patra, R. Grillo, L. S. Acosta-Torres, S. Sharma, H.-S. Shin, L. A. Diaz-Torres, M. K. Swamy and G. Das, J. Nanobiotechnol., 2018, 16, 1-33.

307 J. Deng, Y. Mo, J. Liu, R. Guo, Y. Zhang, W. Xue and Y. Zhang, J. Nanosci. Nanotechnol., 2016, 16, 5740-5745. 
308 D. Liu, Y. Zhang, G. Jiang, W. Yu, B. Xu and J. Zhu, ACS Biomater. Sci. Eng., 2018, 4, 1687-1695.

309 V. A. Ovsyannikov, M. V. Zamoryanskaya, A. V. Semencha, K. A. Lycheva, T. S. Kol'tsova, O. V. Tolochko and L. N. Blinov, Glass Phys. Chem., 2015, 41, 533-536.

310 E. Blanco, H. Shen and M. Ferrari, Nat. Biotechnol., 2015, 33, 941-951.

311 K. Bogusz, M. Tehei, C. Stewart, M. McDonald, D. Cardillo, M. Lerch, S. Corde, A. Rosenfeld, H. K. Liu and K. Konstantinov, RSC Adv., 2014, 4, 24412.

312 Y. Lu, L. Li, Z. Lin, M. Li, X. Hu, Y. Zhang, M. Peng, H. Xia and G. Han, Adv. Healthcare Mater., 2018, 7, 1800602.

313 L. Li, Y. Lu, C. Jiang, Y. Zhu, X. Yang, X. Hu, Z. Lin, Y. Zhang, M. Peng, H. Xia and C. Mao, Adv. Funct. Mater., 2018, 28, 1704623.

314 Z. Song, Y. Chang, H. Xie, X.-F. Yu, P. K. Chu and T. Chen, NPG Asia Mater., 2017, 9, e439-e439.

315 J. Deng, X. Xun, W. Zheng, Y. Su, L. Zheng, C. Wang and M. Su, J. Mater. Chem. B, 2018, 6, 7966-7973.

316 B. R. Smith and S. S. Gambhir, Chem. Rev., 2017, 117, 901-986.

317 S. Chapman, M. Dobrovolskaia, K. Farahani, A. Goodwin, A. Joshi, H. Lee, T. Meade, M. Pomper, K. Ptak, J. Rao, R. Singh, S. Sridhar, S. Stern, A. Wang, J. B. Weaver, G. Woloschak and L. Yang, Nano Today, 2013, 8, 454-460.

318 L. K. Bogart, G. Pourroy, C. J. Murphy, V. Puntes, T. Pellegrino, D. Rosenblum, D. Peer and R. Lévy, ACS Nano, 2014, 8, 3107-3122.

319 X. Han, K. Xu, O. Taratula and K. Farsad, Nanoscale, 2019, 11, 799-819.

320 G. Ma, X. Liu, G. Deng, H. Yuan, Q. Wang and J. Lu, J. Mater. Chem. B, 2018, 6, 6788-6795.

321 M. F. Attia, J. Wallyn, N. Anton and T. F. Vandamme, Crit. Rev. Ther. Drug Carrier Syst., 2018, 35, 391-431.

322 L. Robison, L. Zhang, R. J. Drout, P. Li, C. R. Haney, A. Brikha, H. Noh, B. L. Mehdi, N. D. Browning, V. P. Dravid, Q. Cui, T. Islamoglu and O. K. Farha, ACS Appl. Bio Mater., 2019, 2, 1197-1203.

323 M. Hernández-Rivera, S. Y. Cho, S. E. Moghaddam, B. Y. Cheong, M. D. G. Cabreira-Hansen, J. T. Willerson, E. C. Perin and L. J. Wilson, Contrast Media Mol. Imaging, 2019, 2183051.

324 D. W. Bierer, Rev. Infect. Dis., 1990, 12, S3-S8.

325 Z. Li, K. Ai, Z. Yang, T. Zhang, J. Liu and X. Cui, RSC Adv., 2017, 7, 29672-29678.

326 X. Lu, Y. Li, X. Bai, G. Hu and L. Wang, Sci. China Mater., 2017, 60, 777-788.

327 S. Chakravarty, J. Unold, D. D. Shuboni-Mulligan, B. Blanco-Fernandez and E. M. Shapiro, Nanoscale, 2016, 8, 13217-13222.

328 J. Li, F. Jiang, B. Yang, X. R. Song, Y. Liu, H. H. Yang, D. R. Cao, W. R. Shi and G. N. Chen, Sci. Rep., 2013, 3, 1998.

329 D. Kim, S. Park, J. H. Lee, Y. Y. Jeong and S. Jon, J. Am. Chem. Soc., 2007, 129, 7661-7665.

330 H. Ke, X. Yue, J. Wang, S. Xing, Q. Zhang, Z. Dai, J. Tian, S. Wang and Y. Jin, Small, 2014, 10, 1220-1227.
331 L. Cheng, C. Yuan, S. Shen, X. Yi, H. Gong, K. Yang and Z. Liu, ACS Nano, 2015, 9, 11090-11101.

332 L. Cheng, J. Liu, X. Gu, H. Gong, X. Shi, T. Liu, C. Wang, X. Wang, G. Liu, H. Xing, W. Bu, B. Sun and Z. Liu, Adv. Mater., 2014, 26, 1886-1893.

333 Y. Jin, Y. Li, X. Ma, Z. Zha, L. Shi, J. Tian and Z. Dai, Biomaterials, 2014, 35, 5795-5804.

334 H. Bi, F. He, Y. Dong, D. Yang, Y. Dai, L. Xu, R. Lv, S. Gai, P. Yang and J. Lin, Chem. Mater., 2018, 30, 3301-3307.

335 P. Lei, R. An, P. Zhang, S. Yao, S. Song, L. Dong, X. Xu, K. Du, J. Feng and H. Zhang, Adv. Funct. Mater., 2017, 27, 1702018.

336 G. Ku, M. Zhou, S. Song, Q. Huang, J. Hazle and C. Li, ACS Nano, 2012, 6, 7489-7496.

337 S. Park, G. Park, J. Kim, W. Choi, U. Jeong and C. Kim, Nanoscale, 2018, 10, 20548-20558.

338 A. De La Zerda, C. Zavaleta, S. Keren, S. Vaithilingam, S. Bodapati, Z. Liu, J. Levi, B. R. Smith, T. J. Ma, O. Oralkan, Z. Cheng, X. Chen, H. Dai, B. T. Khuri-Yakub and S. S. Gambhir, Nat. Nanotechnol., 2008, 3, 557-562.

339 L. V. Wang and S. Hu, Science, 2012, 335, 1458-1462.

340 X. Cheng, Y. Yong, Y. Dai, X. Song, G. Yang, Y. Pan and C. Ge, Theranostics, 2017, 7, 4087-4098.

341 C. M. Hessel, V. P. Pattani, M. Rasch, M. G. Panthani, B. Koo, J. W. Tunnell and B. A. Korgel, Nano Lett., 2011, 11, 2560-2566.

342 Y. Shi, H. Qin, S. Yang and D. Xing, Nano Res., 2016, 9, 3644-3655.

343 Y. Li, Y. Sun, T. Cao, Q. Su, Z. Li, M. Huang, R. Ouyang, H. Chang, S. Zhang and Y. Miao, Nanoscale, 2017, 9, 14364-14375.

344 Z. Li, X. Fan, J. Liu, Y. Hu, Y. Yang, Z. Li, Y. Sun, C. Chen and M. Yu, Nanomedicine, 2018, 13, 2283-2300.

345 Z. Li, Y. Hu, K. A. A. Howard, T. Jiang, X. Fan, Z. Miao, Y. Sun, F. Besenbacher and M. Yu, ACS Nano, 2016, 10, 984-997.

346 E. Terreno, D. D. Castelli, A. Viale and S. Aime, Chem. Rev., 2010, 110, 3019-3042.

347 P. Caravan, J. J. Ellison, T. J. McMurry and R. B. Lauffer, Chem. Rev., 1999, 99, 2293-2352.

348 G. Song, C. Liang, H. Gong, M. Li, X. Zheng, L. Cheng, K. Yang, X. Jiang and Z. Liu, Adv. Mater., 2015, 27, 6110-6117.

349 Y. Lu, L. Zhang, J. Li, Y. De Su, Y. Liu, Y. J. Xu, L. Dong, H. L. Gao, J. Lin, N. Man, P. F. Wei, W. P. Xu, S. H. Yu and L. P. Wen, Adv. Funct. Mater., 2013, 23, 1534-1546.

350 N. Lee, H. R. Cho, M. H. Oh, S. H. Lee, K. Kim, B. H. Kim, K. Shin, T. Y. Ahn, J. W. Choi, Y. W. Kim, S. H. Choi and T. Hyeon, J. Am. Chem. Soc., 2012, 134, 10309-10312.

351 Y. Dai, C. Wu, S. Wang, Q. Li, M. Zhang, J. Li and K. Xu, Nanomedicine, 2018, 14, 547-555.

352 Y. Sun, Y. Zheng, H. Ran, Y. Zhou, H. Shen, Y. Chen, H. Chen, T. M. Krupka, A. Li, P. Li, Z. Wang and Z. Wang, Biomaterials, 2012, 33, 5854-5864.

353 Y. Huang, M. Ma, S. Chen, J. Dai, F. Chen and Z. Wang, RSC Adv., 2014, 4, 26861-26865. 
354 K. Yang, S. Zhang, G. Zhang, X. Sun, S. T. Lee and Z. Liu, Nano Lett., 2010, 10, 3318-3323.

355 J. R. Melamed, R. S. Edelstein and E. S. Day, ACS Nano, 2015, 9, 6-11.

356 A. M. Smith, M. C. Mancini and S. Nie, Nat. Nanotechnol., 2009, 4, 710-711.

357 K. Welsher, S. P. Sherlock and H. Dai, Proc. Natl. Acad. Sci. U. S. A., 2011, 108, 8943-8948.

358 Z. Xiao, C. Xu, X. Jiang, W. Zhang, Y. Peng, R. Zou, X. Huang, Q. Liu, Z. Qin and J. Hu, Nano Res., 2016, 9, 1934-1947.

359 H. Zhou, L. Che, Z. Guo, M. Wu, W. Li, W. Xu and L. Liu, ACS Sustainable Chem. Eng., 2018, 6, 4863-4870.

360 Y. Zhang, H. Zhang, Y. Wang, H. Wu, B. Zeng, Y. Zhang, Q. Tian and S. Yang, J. Mater. Chem. B, 2017, 5, 1846-1855.

361 G. Yu, A. Liu, H. Jin, Y. Chen, D. Yin, R. Huo, S. Wang and J. Wang, J. Phys. Chem. C, 2018, 122, 3794-3800.

362 Y. Cheng, Y. Chang, Y. Feng, H. Jian, Z. Tang and H. Zhang, Angew. Chem., Int. Ed., 2018, 57, 246-251.

363 H. Xie, Z. Li, Z. Sun, J. Shao, X. F. Yu, Z. Guo, J. Wang, Q. Xiao, H. Wang, Q. Q. Wang, H. Zhang and P. K. Chu, Small, 2016, 12, 4136-4145.

364 P. Carmeliet and R. K. Jain, Nat. Rev. Drug Discovery, 2011, 10, 417-427.

365 A. Schroeder, D. A. Heller, M. M. Winslow, J. E. Dahlman, G. W. Pratt, R. Langer, T. Jacks and D. G. Anderson, Nat. Rev. Cancer, 2012, 12, 39-50.

366 C. Liang, S. Diao, C. Wang, H. Gong, T. Liu, G. Hong, X. Shi, H. Dai and Z. Liu, Adv. Mater., 2014, 26, 5646-5652.

367 C. Wang, L. Xu, C. Liang, J. Xiang, R. Peng and Z. Liu, Adv. Mater., 2014, 26, 8154-8162.

368 Z. Li, J. Shao, Q. Luo, X.-F. Yu, H. Xie, H. Fu, S. Tang, H. Wang, G. Han and P. K. Chu, Biomaterials, 2017, 133, 37-48.

369 G. Song, C. Liang, X. Yi, Q. Zhao, L. Cheng, K. Yang and Z. Liu, Adv. Mater., 2016, 28, 2716-2723.

370 L. Milane, S. Ganesh, S. Shah, Z.-F. Duan and M. Amiji, J. Controlled Release, 2011, 155, 237-247.

371 H. Cao, Z. Dan, X. He, Z. Zhang, H. Yu, Q. Yin and Y. Li, ACS Nano, 2016, 10, 7738-7748.

372 H. Zhao, L. Li, J. Zhang, C. Zheng, K. Ding, H. Xiao, L. Wang and Z. Zhang, ACS Appl. Mater. Interfaces, 2018, 10, 31124-31135.

373 K. Wang, J. Zhuang, L. Chen, D. Xu, X. Zhang, Z. Chen, Y. Wei and Y. Zhang, Colloids Surf., B, 2017, 160, 297-304.

374 Z. Guo, Y. Liu, H. Zhou, K. Zheng, D. Wang, M. Jia, P. Xu, K. Ma, C. Cui and L. Wang, Colloids Surf., B, 2019, 184, 110546.

375 H. Zhao, J. Wang, X. Kong, E. Li, Y. Liu, X. Du, Z. Kang, Y. Tang, Y. Kuang, Z. Yang, Y. Zhou and Q. Wang, Sci. Rep., 2016, 6, 29719.

376 G. Solinas, G. Germano, A. Mantovani and P. Allavena, J. Leukocyte Biol., 2009, 86, 1065-1073.

377 A. Sica, T. Schioppa, A. Mantovani and P. Allavena, Eur. J. Cancer, 2006, 42, 717-727.

378 A. Mantovani, T. Schioppa, C. Porta, P. Allavena and A. Sica, Cancer Metastasis Rev., 2006, 25, 315-322.
379 T. Bauleth-Ramos, M. A. Shahbazi, D. Liu, F. Fontana, A. Correia, P. Figueiredo, H. Zhang, J. P. Martins, J. T. Hirvonen, P. Granja, B. Sarmento and H. A. Santos, Adv. Funct. Mater., 2017, 27, 1703303.

380 W. R. Chen, A. K. Singhal, H. Liu and R. E. Nordquist, Cancer Res., 2001, 61, 459-461.

381 L. Song, X. Dong, S. Zhu, C. Zhang, W. Yin, X. Zhang, X. Liu and Z. Gu, Adv. Healthcare Mater., 2018, 7, 1800830.

382 X. Zhang, J. Du, Z. Guo, J. Yu, Q. Gao, W. Yin, S. Zhu, Z. Gu and Y. Zhao, Adv. Sci., 2019, 6, 1801122.

383 M. Kowalik, J. Masternak and B. Barszcz, Curr. Med. Chem., 2019, 26, 729-759.

384 J. Du, X. Wang, X. Dong, C. Zhang, L. Mei, Y. Zang, L. Yan, H. Zhang and Z. Gu, Nanoscale, 2019, 11, 7157-7165.

385 A. Rajaee, S. Wang, L. Zhao, D. Wang, Y. Liu, J. Wang and K. Ying, Phys. Med. Biol., 2019, 64, 195007.

386 M. Alqathami, A. Blencowe, U. J. Yeo, R. Franich, S. Doran, G. Qiao and M. Geso, Journal of Physics: Conference Series, Institute of Physics Publishing, 2013, vol. 444.

387 L. Jiao, Q. Li, J. Deng, N. Okosi, J. Xia and M. Su, Nanoscale, 2018, 10, 6751-6757.

388 M. Hossain and M. Su, J. Phys. Chem. C, 2012, 116, 23047-23052.

389 X.-D. Zhang, D. Wu, X. Shen, J. Chen, Y.-M. Sun, P.-X. Liu and X.-J. Liang, Biomaterials, 2012, 33, 6408-6419.

390 L. Zhang, Q. Chen, X. Zou, J. Chen, L. Hu, Z. Dong, J. Zhou, Y. Chen, Z. Liu and L. Cheng, J. Mater. Chem. B, 2019, 7, 5170-5181.

391 S. S. Sabharwal and P. T. Schumacker, Nat. Publ. Gr., 2014, 14, 709-721.

392 M. H. Lee, Z. Yang, C. W. Lim, Y. H. Lee, S. Dongbang, C. Kang and J. S. Kim, Chem. Rev., 2013, 113, 5071-5109.

393 H. Lin, Y. Chen and J. Shi, Chem. Soc. Rev., 2018, 47, 1938-1958.

394 M.-A. Shahbazi, P. V. Almeida, A. Correia, B. Herranz-Blanco, N. Shrestha, E. Mäkilä, J. Salonen, J. Hirvonen and H. A. Santos, J. Controlled Release, 2017, 249, 111-122.

395 J. M. Estrela, A. Ortega, S. Mena, J. A. Sirerol and E. Obrador, Crit. Rev. Clin. Lab. Sci., 2016, 53, 253-267.

396 J. Wang and J. Yi, Cancer Biol. Ther., 2008, 7, 1875-1884.

397 Q. Xiang, J. Yu and M. Jaroniec, Chem. Soc. Rev., 2012, 41, 782-796.

398 S. S. Wang and G. Y. Yang, Chem. Rev., 2015, 115, 4893-4962.

399 R. Costi, A. E. Saunders, E. Elmalem, A. Salant and U. Banin, Nano Lett., 2008, 8, 637-641.

400 X. Yu, A. Shavel, X. An, Z. Luo, M. Ibáñez and A. Cabot, J. Am. Chem. Soc., 2014, 136, 9236-9239.

401 B. K. Patra, A. Shit, A. K. Guria, S. Sarkar, G. Prusty and N. Pradhan, Chem. Mater., 2015, 27, 650-657.

402 Y. Zang, L. Gong, L. Mei, Z. Gu and Q. Wang, ACS Appl. Mater. Interfaces, 2019, 11, 18942-18952.

403 X. Wang, C. Zhang, J. Du, X. Dong, S. Jian, L. Yan, Z. Gu and Y. Zhao, ACS Nano, 2019, 13, 5947-5958.

404 J. A. Barreto, W. O'Malley, M. Kubeil, B. Graham, H. Stephan and L. Spiccia, Adv. Mater., 2011, 23, H18-H40. 
405 A. Jakhmola, N. Anton and T. F. Vandamme, Adv. Healthcare Mater., 2012, 1, 413-431.

406 Z. Li, Y. Hu, Z. Miao, H. Xu, C. Li, Y. Zhao, Z. Li, M. Chang, Z. Ma, Y. Sun, F. Besenbacher, P. Huang and M. Yu, Nano Lett., 2018, 18, 6778-6788.

407 F. Mao, L. Wen, C. Sun, S. Zhang, G. Wang, J. Zeng, Y. Wang, J. Ma, M. Gao and Z. Li, ACS Nano, 2016, 10, 11145-11155.

408 X. D. Zhang, J. Yang, S. S. Song, W. Long, J. Chen, X. Shen, H. Wang, Y. M. Sun, P. X. Liu and S. Fan, Int. J. Nanomed., 2014, 9, 2069-2072.

409 X. Lv, X. Wang, T. Li, C. Wei, Y. Tang, T. Yang, Q. Wang, X. Yang, H. Chen, J. Shen, H. Yang and H. Ke, Small, 2018, 14, 1802904.

410 Y. Song, Y. Wang, S. Wang, Y. Cheng, Q. Lu, L. Yang, F. Tan and N. Li, Nano Res., 2019, 12, 1770-1780.

411 J. Bai, X. Jia, Y. Ruan, C. Wang and X. Jiang, Inorg. Chem., 2018, 57, 10180-10188.

412 J. Cheng, W. Wang, X. Xu, Z. Lin, C. Xie, Y. Zhang, T. Zhang, L. Li, Y. Lu and Q. Li, Mater. Sci. Eng., C, 2020, 107, 110324.

413 H. Yang, Y. Zhang, J. Song, M. Wang, S. Yu, L. Chen, X. Li, S. Yang and L. Yang, Chem. Eng. J., 2019, 375, 121941.

414 C. Yang, Y. Chen, W. Guo, Y. Gao, C. Song, Q. Zhang, N. Zheng, X. Han and C. Guo, Adv. Funct. Mater., 2018, 28, 1706827.

415 Y. Cheng, Y. Chang, Y. Feng, H. Jian, X. Wu, R. Zheng, K. Xu and H. Zhang, Adv. Mater., 2019, 31, 1806808.

416 X. Li, Y. Liu, F. Fu, M. Cheng, Y. Liu, L. Yu, W. Wang, Y. Wan and Z. Yuan, Nano-Micro Lett., 2019, 11, 68.

417 Y. Song, Y. Wang, Y. Zhu, Y. Cheng, Y. Wang, S. Wang, F. Tan, F. Lian and N. Li, Adv. Healthcare Mater., 2019, 8, 1900250.

418 S. Wang, X. Li, Y. Chen, X. Cai, H. Yao, W. Gao, Y. Zheng, X. An, J. Shi and H. Chen, Adv. Mater., 2015, 27, 2775-2782.

419 Q. Cao, X. Guo, W. Zhang, G. Guan, X. Huang, S. A. He, M. Xu, R. Zou, X. Lu and J. Hu, Dalton Trans., 2019, 48, 3360-3368.

420 L. Li, Y. Lu, Z. Lin, A. S. Mao, J. Jiao, Y. Zhu, C. Jiang, Z. Yang, M. Peng and C. Mao, Mater. Horiz., 2019, 6, 1845-1853.

421 C. Liu, L. Zhang, X. Chen, S. Li, Q. Han, L. Li and C. Wang, Chem. Eng. J., 2019, 122720.

422 M. Mohammadniaei, T. Lee, B. G. Bharate, J. Yoon, H. K. Choi, S. Jeong Park, J. Kim, J. Kim and J. W. Choi, Small, 2018, 14, e1802934.

423 L. Feng, D. Yang, S. Gai, F. He, G. Yang, P. Yang and J. Lin, Chem. Eng. J., 2018, 351, 1147-1158.

424 Z. Guo, S. Zhu, Y. Yong, X. Zhang, X. Dong, J. Du, J. Xie, Q. Wang, Z. Gu and Y. Zhao, Adv. Mater., 2017, 29, 1704136.

425 S. K. Metkar and K. Girigoswami, Biocatal. Agric. Biotechnol., 2019, 17, 271-283.

426 W.-W. Zhao, J.-J. Xu and H.-Y. Chen, Chem. Rev., 2014, 114, 7421-7441.

427 S.-Y. Yu, L. Zhang, L.-B. Zhu, Y. Gao, G.-C. Fan, D.-M. Han, G. Chen and W.-W. Zhao, Coord. Chem. Rev., 2019, 393, 9-20.
428 A. P. F. Turner, Chem. Soc. Rev., 2013, 42, 3184.

429 M. Holzinger, A. Le Goff and S. Cosnier, Front. Chem., 2014, 2,63 .

430 A. Munawar, R. Schirhagl, A. Rehman, A. Shaheen, A. Taj, K. Bano, N. J. Bassous, T. J. Webster, W. S. Khan and S. Z. Bajwa, J. Hazard. Mater., 2019, 373, 50-59.

431 J. Wang, Electroanalysis, 2005, 17, 1341-1346.

432 M. Cadevall, J. Ros and A. Merkoçi, Functional and Physical Properties of Polymer Nanocomposites, John Wiley \& Sons, Ltd, Chichester, UK, 2016, pp. 159-181.

433 S.-N. Ding, D. Shan, H.-G. Xue and S. Cosnier, Bioelectrochemistry, 2010, 79, 218-222.

434 J. Krüger, P. Winkler, E. Lüderitz, M. Lück and H. U. Wolf, Ullmann's Encyclopedia of Industrial Chemistry, Wiley-VCH Verlag GmbH \& Co. KGaA, Weinheim, Germany, 2003.

435 W.-W. Zhao, J.-J. Xu and H.-Y. Chen, Chem. Soc. Rev., 2015, 44, 729-741.

436 M. Adamovski, A. Zając, P. Gründler and G.-U. Flechsig, Electrochem. Commun., 2006, 8, 932-936.

437 R. Pauliukaitè, S. B. Hočevar, B. Ogorevc and J. Wang, Electroanalysis, 2004, 16, 719-723.

438 C. I. Fort, L. C. Cotet, A. Vulpoi, G. L. Turdean, V. Danciu, L. Baia and I. C. Popescu, Sens. Actuators, B, 2015, 220, 712-719.

439 N. A. Malakhova, A. A. Mysik, S. Y. Saraeva, N. Y. Stozhko, M. A. Uimin, A. E. Ermakov and K. Z. Brainina, J. Anal. Chem., 2010, 65, 640-647.

440 M. Á. G. Rico, M. Olivares-Marín and E. P. Gil, Talanta, 2009, 80, 631-635.

441 A. P. Periasamy, S. Yang and S.-M. Chen, Talanta, 2011, 87, 15-23.

442 S. Taufik, N. A. Yusof, T. W. Tee and I. Ramli, Int. J. Electrochem. Sci., 2011, 6, 1880-1891.

443 Z. Mohammed, T. Tan Wee, A. H. Abdullah, Z. Zainal and G. J. Kheng, Int. J. Electrochem. Sci., 2011, 6, 279-288.

444 H. Yin, B. Sun, Y. Zhou, M. Wang, Z. Xu, Z. Fu and S. Ai, Biosens. Bioelectron., 2014, 51, 103-108.

445 X. Jia, J. Li and E. Wang, Electroanalysis, 2010, 22, 1682-1687. 446 V. Urbanová, M. Bartoš, K. Vytřas and A. Kuhn, Electroanalysis, 2010, 22, 1524-1530.

447 H. Zhou, H. Hou, L. Dai, Y. Li, J. Zhu and L. Wang, Chin. J. Chem., 2016, 24, 410-414.

448 V. Fruth, M. Popa, D. Berger, R. Ramer, M. Gartner, A. Ciulei and M. Zaharescu, J. Eur. Ceram. Soc., 2005, 25, 2171-2174.

449 I. Švancara, C. Prior, S. B. Hočevar and J. Wang, Electroanalysis, 2010, 22, 1405-1420.

450 V. Jovanovski, S. B. Hočevar and B. Ogorevc, Curr. Opin. Electrochem., 2017, 3, 114-122.

451 R. L. Thayer, C. A. Randall and S. Trolier-McKinstry, J. Appl. Phys., 2003, 94, 1941-1947.

452 H. T. Fan, X. M. Teng, S. S. Pan, C. Ye, G. H. Li and L. D. Zhang, Appl. Phys. Lett., 2005, 87, 231916.

453 L. Leontie, M. Caraman, M. Alexe and C. Harnagea, Surf. Sci., 2002, 507-510, 480-485.

454 E. Kanazawa, G. Sakai, K. Shimanoe, Y. Kanmura, Y. Teraoka, N. Miura and N. Yamazoe, Sens. Actuators, B, 2001, 77, 72-77. 
455 N. C. Miller and M. Bernechea, APL Mater., 2018, 6, 084503. 456 S. Liu, S. Zhao, W. Tu, X. Wang, X. Wang, J. Bao, Y. Wang, M. Han and Z. Dai, Chem. - Eur. J., 2018, 24, 3638.

457 D. Fan, H. Wang, M. S. Khan, C. Bao, H. Wang, D. Wu, Q. Wei and B. Du, Biosens. Bioelectron., 2017, 97, 253-259. 458 H.-Y. Jiang, J. Liu, K. Cheng, W. Sun and J. Lin, J. Phys. Chem. C, 2013, 117, 20029-20036.

459 N. Wetchakun, S. Chaiwichain, B. Inceesungvorn, K. Pingmuang, S. Phanichphant, A. I. Minett and J. Chen, ACS Appl. Mater. Interfaces, 2012, 4, 3718-3723.

460 L. Ge, Y. Xu, L. Ding, F. You, Q. Liu and K. Wang, Biosens. Bioelectron., 2019, 124-125, 33-39.

461 Q. Han, R. Wang, B. Xing, H. Chi, D. Wu and Q. Wei, Biosens. Bioelectron., 2018, 106, 7-13.

462 L.-B. Zhu, L. Lu, H.-Y. Wang, G.-C. Fan, Y. Chen, J.-D. Zhang and W.-W. Zhao, Biosens. Bioelectron., 2019, 140, 111349.

463 G.-L. Wang, J.-J. Xu, H.-Y. Chen and S.-Z. Fu, Biosens. Bioelectron., 2009, 25, 791-796.

464 D. Fan, C. Bao, X. Liu, J. Feng, D. Wu, H. Ma, H. Wang, Q. Wei and B. Du, Talanta, 2019, 198, 417-423.

465 R. Li, H. Ren, W. Ma, S. Hong, L. Wu and Y. Huang, Catal. Commun., 2018, 106, 1-5.

466 H. Yu, B. Huang, H. Wang, X. Yuan, L. Jiang, Z. Wu, J. Zhang and G. Zeng, J. Colloid Interface Sci., 2018, 522, 82-94.

467 M. Du, Y. Du, Y. Feng, K. Yang, X. Lv, N. Jiang and Y. Liu, Carbohydr. Polym., 2018, 195, 393-400.

468 P. Yan, D. Jiang, Y. Tian, L. Xu, J. Qian, H. Li, J. Xia and H. Li, Biosens. Bioelectron., 2018, 111, 74-81.

469 L. Li, L. Ai, C. Zhang and J. Jiang, Nanoscale, 2014, 6, 4627.

470 P. Yan, L. Xu, D. Jiang, H. Li, J. Xia, Q. Zhang, M. Hua and H. Li, Electrochim. Acta, 2018, 259, 873-881.

471 J. Guozhi, W. Peng, Z. Yanbang and C. Kai, Sci. Rep., 2016, 6, 25884.

472 K. Kadel, L. Kumari, W. Z. Li, J. Y. Huang and P. P. Provencio, Nanoscale Res. Lett., 2010, 6, 57.

473 P. Di Pietro, M. Ortolani, O. Limaj, A. Di Gaspare, V. Giliberti, F. Giorgianni, M. Brahlek, N. Bansal, N. Koirala, S. Oh, P. Calvani and S. Lupi, Nat. Nanotechnol., 2013, 8, 556-560.

474 G. Martinez, B. A. Piot, M. Hakl, M. Potemski, Y. S. Hor, A. Materna, S. G. Strzelecka, A. Hruban, O. Caha, J. Novák, A. Dubroka, Č. Drašar and M. Orlita, Sci. Rep., 2017, 7, 6891.

475 D. Hsieh, Y. Xia, D. Qian, L. Wray, F. Meier, J. H. Dil, J. Osterwalder, L. Patthey, A. V. Fedorov, H. Lin, A. Bansil, D. Grauer, Y. S. Hor, R. J. Cava and M. Z. Hasan, Phys. Rev. Lett., 2009, 103, 146401.

476 S. Wu, G. Liu, P. Li, H. Liu and H. Xu, Biosens. Bioelectron., 2012, 38, 289-294.

477 S. Chen, Y.-M. Fang, J. Li, J.-J. Sun, G.-N. Chen and H.-H. Yang, Biosens. Bioelectron., 2013, 46, 171-174.

478 M. Z. Hossain, S. L. Rumyantsev, D. Teweldebrhan, K. M. F. Shahil, M. Shur and A. A. Balandin, Phys. Status Solidi, 2011, 208, 144-146.

479 P. Roushan, J. Seo, C. V. Parker, Y. S. Hor, D. Hsieh, D. Qian, A. Richardella, M. Z. Hasan, R. J. Cava and A. Yazdani, Nature, 2009, 460, 1106-1109.
480 M. Mohammadniaei, J. Yoon, T. Lee, B. G. Bharate, J. Jo, D. Lee and J.-W. Choi, Small, 2018, 14, 1703970.

481 X. Meng, Z. Xu, M. Wang, H. Yin and S. Ai, Anal. Methods, 2012, 4, 1736.

482 Y. P. Dong, L. Huang, J. Zhang, X. F. Chu and Q. F. Zhang, Electrochim. Acta, 2012, 74, 189-193.

483 X. Wang, T. Yan, Y. Li, Y. Liu, B. Du, H. Ma and Q. Wei, Sci. Rep., 2015, 5, 17945.

484 A. K. Dutta, S. K. Maji, K. Mitra, A. Sarkar, N. Saha, A. B. Ghosh and B. Adhikary, Sens. Actuators, B, 2014, 192, 578-585.

485 A. A. Tahir, M. A. Ehsan, M. Mazhar, K. G. U. Wijayantha, M. Zeller and A. D. Hunter, Chem. Mater., 2010, 22, 5084-5092.

486 L. Cui, J. Hu, M. Wang, X. Diao, C. Li and C. Zhang, Anal. Chem., 2018, 90, 11478-11485.

487 X. Li, D. Wu, H. Ma, H. Wang, Y. Wang, D. Fan, B. Du, Q. Wei and N. Zhang, Biosens. Bioelectron., 2019, 131, 136-142.

488 W. Huang, C. Xing, Y. Wang, Z. Li, L. Wu, D. Ma, X. Dai, Y. Xiang, J. Li, D. Fan and H. Zhang, Nanoscale, 2018, 10, 2404-2412.

489 C. Ye, M. Q. Wang, Z. F. Gao, Y. Zhang, J. L. Lei, H. Q. Luo and N. B. Li, Anal. Chem., 2016, 88, 11444-11449.

490 B. Sun, F. Qiao, L. Chen, Z. Zhao, H. Yin and S. Ai, Biosens. Bioelectron., 2014, 54, 237-243.

491 O. K. Okoth, K. Yan, Y. Liu and J. Zhang, Biosens. Bioelectron., 2016, 86, 636-642.

492 F. You, M. Zhu, L. Ding, Y. Xu and K. Wang, Biosens. Bioelectron., 2019, 130, 230-235.

493 B. Wang, J.-T. Cao, Y.-X. Dong, F.-R. Liu, X.-L. Fu, S.-W. Ren, S.-H. Ma and Y.-M. Liu, Chem. Commun., 2018, 54, 806-809.

494 W. Yan, X. Feng, X. Chen, X. Li and J.-J. Zhu, Bioelectrochemistry, 2008, 72, 21-27.

495 G. Cai, J. Tu, D. Zhou, J. Zhang, Q. Xiong, X. Zhao, X. Wang and C. Gu, J. Phys. Chem. C, 2013, 117, 15967-15975.

496 Q. Zhu, F. Gao, Y. Yang, B. Zhang, W. Wang, Z. Hu and Q. Wang, Sens. Actuators, B, 2015, 207, 819-826.

497 Y. Fuseya, M. Ogata and H. Fukuyama, J. Phys. Soc. Jpn., 2015, 84, 012001.

498 B. O’Brien, M. Plaza, L. Y. Zhu, L. Perez, C. L. Chien and P. C. Searson, J. Phys. Chem. C, 2008, 112, 12018-12023.

499 F. Y. Yang, Science, 1999, 284, 1335-1337.

500 R. Fu, S. Xu, Y. N. Lu and J. J. Zhu, Cryst. Growth Des., 2005, 5, 1379-1385.

501 Y. Bu, Z. Chen, W. Li and B. Hou, ACS Appl. Mater. Interfaces, 2013, 5, 12361-12368.

502 J. Mohanty, N. Barooah, V. Dhamodharan, S. Harikrishna, P. I. Pradeepkumar and A. C. Bhasikuttan, J. Am. Chem. Soc., 2013, 135, 367-376.

503 Ü. Anik, M. Çubukçu, S. Çevik and S. Timur, Electroanalysis, 2010, 22, 1519-1523.

504 R. María-Hormigos, M. J. Gismera, M. T. Sevilla, Á. Rumbero and J. R. Procopio, Electroanalysis, 2017, 29, 60-66. 
505 C. Dossi, D. Monticelli, A. Pozzi and S. Recchia, Biosensors, 2016, 6, 38.

506 M. Khairy, R. O. Kadara, D. K. Kampouris and C. E. Banks, Anal. Methods, 2010, 2, 645.

507 H.-L. Fang, H.-X. Zheng, M.-Y. Ou, Q. Meng, D.-H. Fan and W. Wang, Sens. Actuators, B, 2011, 153, 369-372.

508 T. Romih, S. B. Hočevar, A. Jemec and D. Drobne, Electrochim. Acta, 2016, 188, 393-397.

509 H. Sopha, S. B. Hocevar, B. Pihlar and B. Ogorevc, Electrochim. Acta, 2012, 60, 274-277.

510 C. A. de Lima and A. Spinelli, Electrochim. Acta, 2013, 107, 542-548.

511 V. Guzsvány, Z. Papp, J. Zbiljić, O. Vajdle and M. Rodić, Molecules, 2011, 16, 4451-4466.

512 Ü. A. Kırgöz, S. Marín, M. Pumera, A. Merkoçi and S. Alegret, Electroanalysis, 2005, 17, 881-886.

513 J. Kruusma, C. Banks and R. Compton, Anal. Bioanal. Chem., 2004, 379, 700-706.

514 R. Pauliukaitė and C. M. A. Brett, Electroanalysis, 2005, 17, 1354-1359.

515 A. Królicka and A. Bobrowski, Electrochim. Acta, 2016, 187, 224-233.

516 M. Dehghanzade and E. Alipour, Anal. Methods, 2016, 8, 1995-2004.

517 X. Y. Xie, H. Q. Luo and N. B. Li, J. Electroanal. Chem., 2010, 639, 175-180.

518 C. Kokkinos and A. Economou, Talanta, 2011, 84, 696-701.

519 P. Niu, C. Fernández-Sánchez, M. Gich, C. NavarroHernández, P. Fanjul-Bolado and A. Roig, Microchim. Acta, 2016, 183, 617-623.

520 C. van der Horst, B. Silwana, E. Iwuoha and V. Somerset, J. Electroanal. Chem., 2015, 752, 1-11.

521 B. Nigović, S. Jurić and I. Mitrović, Talanta, 2017, 164, 201-208.

522 B. Nigović, B. Šimunić and S. Hocevar, Electrochim. Acta, 2009, 54, 5678-5683.

523 Ü. Anık, S. Timur, M. Çubukçu and A. Merkoçi, Microchim. Acta, 2008, 160, 269-273.

524 W. Kang, X. Pei, W. Yue, A. Bange, W. R. Heineman and I. Papautsky, Electroanalysis, 2013, 25, 2586-2594.

525 É. S. Sá, P. S. da Silva, C. L. Jost and A. Spinelli, Sens. Actuators, B, 2015, 209, 423-430.

526 A. Shafiee and A. Atala, Annu. Rev. Med., 2017, 68, 29-40.

527 M. N. Rahaman, D. E. Day, B. Sonny Bal, Q. Fu, S. B. Jung, L. F. Bonewald and A. P. Tomsia, Acta Biomater., 2011, 7, 2355-2373.

528 F. Pati, J. Jang, D.-H. Ha, S. Won Kim, J.-W. Rhie, J.-H. Shim, D.-H. Kim and D.-W. Cho, Nat. Commun., 2014, 5, 3935.

529 A. Nourian Dehkordi, F. Mirahmadi Babaheydari, M. Chehelgerdi and S. Raeisi Dehkordi, Stem Cell Res. Ther., 2019, 10, 111.

530 J. M. Seong, B.-C. Kim, J.-H. Park, I. K. Kwon, A. Mantalaris and Y.-S. Hwang, Biomed. Mater., 2010, 5, 062001.

531 M. Marcacci, E. Kon, V. Moukhachev, A. Lavroukov, S. Kutepov, R. Quarto, M. Mastrogiacomo and R. Cancedda, Tissue Eng., 2007, 13, 947-955.
532 P. K. Valonen, F. T. Moutos, A. Kusanagi, M. G. Moretti, B. O. Diekman, J. F. Welter, A. I. Caplan, F. Guilak and L. E. Freed, Biomaterials, 2010, 31, 2193-2200.

533 M. Kuratate, K. Yoshiba, Y. Shigetani, N. Yoshiba, H. Ohshima and T. Okiji, J. Endod., 2008, 34, 970-974.

534 J. Henkel1, M. A. Woodruff, D. R. Epari, R. Steck, V. Glatt, I. C. Dickinson, P. F. M. Choong, M. A. Schuetz and D. W. Hutmacher, Bone Res., 2013, 3, 216-248.

535 S. D’Oronzo, S. Stucci, M. Tucci and F. Silvestris, Cancer Treat. Rev., 2015, 41, 798-808.

536 J. Wang, M. Yang, Y. Zhu, L. Wang, A. P. Tomsia and C. Mao, Adv. Mater., 2014, 26, 4961-4966.

537 N. Zanjanizadeh Ezazi, M. A. Shahbazi, Y. V. Shatalin, E. Nadal, E. Mäkilä, J. Salonen, M. Kemell, A. Correia, J. Hirvonen and H. A. Santos, Int. J. Pharm., 2018, 536, 241-250.

538 W. Wang and K. W. K. Yeung, Bioact. Mater., 2017, 2, 224-247. 539 K. Zhang, S. Wang, C. Zhou, L. Cheng, X. Gao, X. Xie, J. Sun, H. Wang, M. D. Weir, M. A. Reynolds, N. Zhang, Y. Bai and H. H. K. Xu, Bone Res., 2018, 6, 31.

540 H. Maleki, M. A. Shahbazi, S. Montes, S. H. Hosseini, M. R. Eskandari, S. Zaunschirm, T. Verwanger, S. Mathur, B. Milow, B. Krammer and N. Hüsing, ACS Appl. Mater. Interfaces, 2019, 11, 17256-17269.

541 T. Wu, S. Yang, H. Shi and J. Ye, J. Mater. Sci.: Mater. Med., 2018, 29, 149.

542 A. E. Pazarçeviren, Z. Evis, D. Keskin and A. Tezcaner, Biomed. Mater., 2019, 14, 035018.

543 L. Wang, N. J. Long, L. Li, Y. Lu, M. Li, J. Cao, Y. Zhang, Q. Zhang, S. Xu, Z. Yang, C. Mao and M. Peng, Light: Sci. Appl., 2018, 7, 1.

544 C. Chen, S.-C. Hsieh, N.-C. Teng, C.-K. Kao, S.-Y. Lee, C.-K. Lin and J.-C. Yang, J. Endod., 2014, 40, 251-254.

545 T. J. Webster, E. A. Massa-Schlueter, J. L. Smith and E. B. Slamovich, Biomaterials, 2004, 25, 2111-2121.

546 S. Prasad, I. Ratha, T. Adarsh, A. Anand, P. K. Sinha, P. Diwan, K. Annapurna and K. Biswas, J. Mater. Res., 2018, 33, 178-190.

547 A. E. Pazarçeviren, A. Tahmasebifar, A. Tezcaner, D. Keskin and Z. Evis, Ceram. Int., 2018, 44, 3791-3799.

548 P. K. Vallittu, J. Mater. Sci., 2017, 52, 8772-8784.

549 T. Al Malat, M. Glombitza, J. Dahmen, P.-M. Hax and E. Steinhausen, Z. Orthop. Unfall., 2018, 156, 152-159.

550 Q. Fu, E. Saiz, M. N. Rahaman and A. P. Tomsia, Mater. Sci. Eng., C, 2011, 31, 1245-1256.

551 M. C. Birt, D. W. Anderson, E. Bruce Toby and J. Wang, J. Orthop., 2017, 14, 45-52.

552 I. Allan, H. Newman and M. Wilson, Biomaterials, 2001, 22, 1683-1687.

553 C. Khatua, S. Bodhak, B. Kundu and V. K. Balla, Materialia, 2018, 4, 361-366.

554 E. A. Abou Neel, W. Chrzanowski, V. M. Salih, H.-W. Kim and J. C. Knowles, J. Dent., 2014, 42, 915-928.

555 S. Hosseini, S. Jahangir and M. B. Eslaminejad, Biomater. Oral Dent. Tissue Eng, 2017, 467-501.

556 S. Sharma, D. Srivastava, S. Grover and V. Sharma, J. Clin. Diagn. Res., 2014, 8, 309-315. 
557 M. Venturi, C. Prati, G. Capelli, M. Falconi and L. Breschi, Int. Endod. J., 2003, 36, 54-63.

558 M. Parirokh and M. Torabinejad, J. Endod., 2010, 36, 16-27.

559 S. Bonson, B. G. Jeansonne and T. E. Lallier, J. Dent. Res., 2004, 83, 408-413.

560 A. Nakayama, B. Ogiso, N. Tanabe, O. Takeichi, K. Matsuzaka and T. Inoue, Int. Endod. J., 2005, 38, 203-210.

561 N. Taniishii, N. Hamada, K. Watanabe, Y. Tujimoto, T. Teranaka and T. Umemoto, J. Endod., 2007, 33, 836-839. 562 W. Andelin, S. Shabahang, K. Wright and M. Torabinejad, J. Endod., 2003, 29, 646-650.
563 C. Stewart, K. Konstantinov, M. McDonald, K. Bogusz, D. Cardillo, S. Oktaria, D. Shi, M. Lerch, T. Devers, S. Corde, A. Rosenfeld and M. Tehei, Part. Part. Syst. Charact., 2014, 31, 960-964.

564 M. S. Claro, I. Levy, A. Gangopadhyay, D. J. Smith and M. C. Tamargo, Sci. Rep., 2019, 9, 3370.

565 M. Leng, Y. Yang, Z. Chen, W. Gao, J. Zhang, G. Niu, D. Li, H. Song, J. Zhang, S. Jin and J. Tang, Nano Lett., 2018, 18, 6076-6083.

566 C. Xing, W. Huang, Z. Xie, J. Zhao, D. Ma, T. Fan, W. Liang, Y. Ge, B. Dong, J. Li and H. Zhang, ACS Photonics, 2018, 5, 621-629. 